\title{
Analysis and Optimization of a Dual Free Piston, Spring Assisted, Linear Engine Generator
}

\author{
Matthew C. Robinson
}

Follow this and additional works at: https://researchrepository.wvu.edu/etd

\section{Recommended Citation}

Robinson, Matthew C., "Analysis and Optimization of a Dual Free Piston, Spring Assisted, Linear Engine Generator" (2015). Graduate Theses, Dissertations, and Problem Reports. 6516.

https://researchrepository.wvu.edu/etd/6516

This Dissertation is protected by copyright and/or related rights. It has been brought to you by the The Research Repository @ WVU with permission from the rights-holder(s). You are free to use this Dissertation in any way that is permitted by the copyright and related rights legislation that applies to your use. For other uses you must obtain permission from the rights-holder(s) directly, unless additional rights are indicated by a Creative Commons license in the record and/ or on the work itself. This Dissertation has been accepted for inclusion in WVU Graduate Theses, Dissertations, and Problem Reports collection by an authorized administrator of The Research Repository @ WVU.

For more information, please contact researchrepository@mail.wvu.edu. 


\section{Analysis and Optimization of a Dual Free Piston, Spring Assisted, Linear Engine Generator}

\section{Matthew C. Robinson}

Dissertation Submitted to the

Benjamin M. Statler College of Engineering and Mineral Resources

at West Virginia University

in partial fulfillment of the requirements for the degree of

Doctorate of Philosophy in Mechanical Engineering

Nigel Clark, Ph.D., Chair

Parviz Famouri, Ph.D.

Terence Musho, Ph.D.

Gregory Thompson, Ph.D.

Scott Wayne, Ph.D.

Department of Mechanical and Aerospace Engineering

Morgantown, West Virginia

2015

Keywords: Free Piston, Linear Generator, 2-stroke Linear Engine, $\mathrm{HCCl}$ Combustion, Compression Springs,

Genetic Algorithm Optimization, Free Piston Resonance, Dynamic Control

Copyright 2015 Matthew C. Robinson 


\title{
ABSTRACT
}

\section{Analysis and Optimization of a Dual Free Piston, Spring Assisted, Linear Engine Generator}

\author{
Matthew C. Robinson
}

The free piston linear engine (FPLE) generator has the potential to displace existing crankshaft driven engine technology because of its relative simplicity, higher efficiency, and increased power density. Continued interest in hybrid-electric vehicles for transportation and tightening emissions regulations has created a challenging market for conventional piston engines. Combined with rising market interest in localized power generation means there are exciting opportunities for innovative technologies that can satisfy both regulatory and commercial demands. Many groups around the world are currently working to advance the state of the FPLE, and recent success at West Virginia University will lead to a working prototype device within the next three years.

This dissertation presents the analysis and optimization of a dual free piston, spring assisted, linear engine generator (SALEG). The primary moving part is a dual piston translator driven by 2 -stroke homogeneous charge compression ignition combustion cycles such that the compression stroke for one cylinder corresponds to the expansion stroke of the other. The dynamics of the translator are augmented by the addition of springs that support higher frequency operation, provide energy storage to support cyclic stability, and can be tailored to achieve a desired translator dynamic profile. Current challenges for the device involve optimization for high efficiency performance at steady state and control of the translator position and combustion events.

Using numeric simulation tools in $\mathrm{MATLAB}^{\circledR}$ and Simulink, the dynamic behavior of the translator is modeled in conjunction with the in-cylinder thermodynamics for each engine cylinder and the linear electric alternator load. Sweeps of the primary design parameters explore the design space while demonstrating the interdependency that is characteristic of the FPLE. Then, a genetic algorithm is employed to optimize the SALEG for efficiency based on target power and practical operating constraints. It is demonstrated that low maximum stroke to bore ratio and low intake temperature are favored. Also, the design space becomes more restrictive as target power is raised, but for a range of devices as high as $25 \mathrm{~kW}$, efficiency greater than $40 \%$ can be achieved.

Control mechanisms for the simulated SALEG are demonstrated and compared. These entail the control of alternator force, engine fueling, and intake conditions through the use of proportional and integral control methods. The control methods are applied to achieve resonant start-up of the device and to respond to changes in load demand and misfire. Motored, resonant hot-start is simulated for a device with natural frequency of $40 \mathrm{~Hz}$, and the linear motor and controller parameters are tested. Misfire is shown to lead to rapid loss of compression, so the motored resonant control mechanism is employed to recover after misfire. A map-based controller is used to control intake temperature in response to rapid change in load. For a $50 \%$ reduction in load, intake temperature is raised by $15 \%\left(40{ }^{\circ} \mathrm{C}\right)$ and results in an efficiency drop from $38 \%$ to $22 \%$ at steady state. Ultimately, the simulation tool represents a platform for future investigations where experimental data and more sophisticated modeling techniques might be included to enhance the research and advancement of the free piston linear engine. 


\section{ACKNOWLEDGEMENTS}

First and foremost I would like to thank my wonderful wife for her patience and support through these last months. I could not have met the challenges of this research without her love, laughter, encouragement, and understanding.

Highest regard and appreciation goes to my advisor Dr. Nigel Clark and to my committee members Dr. Terence Musho, Dr. Parviz Famouri, Dr. Greg Thompson, and Dr. Scott Wayne. Their insight and technical help have been instrumental in shaping this work and crucial to my growth as a researcher.

A special thanks goes to Dr. Russ Dean. I could not have had a better mentor and friend throughout the last years. I owe him a debt of gratitude for many of the opportunities that I've been able to pursue, not the least of which being my graduate career.

Finally, thanks to all the many friends, colleagues, instructors, and mentors who have contributed to my personal and professional growth. I look forward to the times when our paths cross. 


\section{TABLE OF CONTENTS}

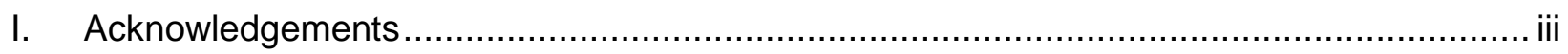

II. Table of Contents....................................................................................................

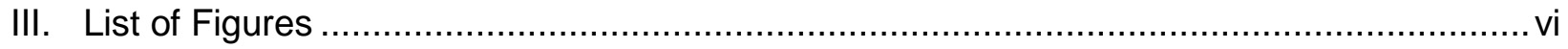

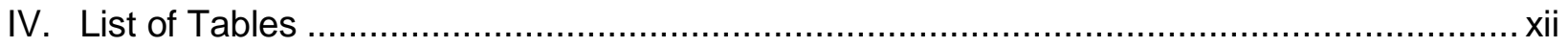

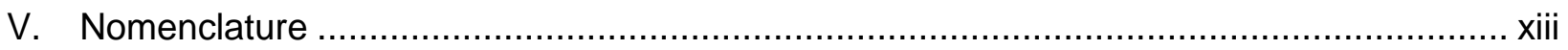

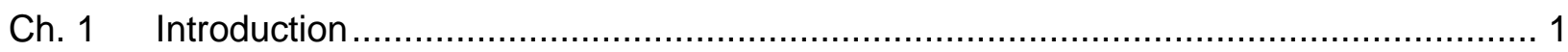

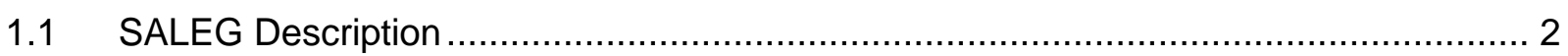

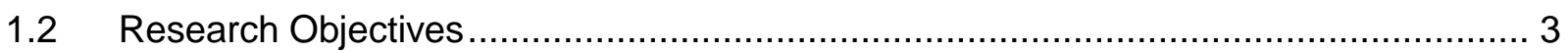

1.2.1 Objective 1: Fundamental and Numerical Simulations ...................................... 4

1.2.2 Objective 2: Parametric Study and Optimization ............................................. 4

1.2.3 Objective 3: Control for Start-up and Transient Response ................................. 5

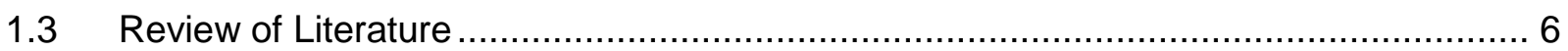

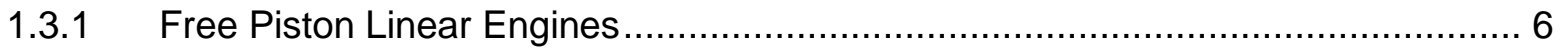

1.3.2 Linear Electric Machine .........................................................................11

1.3.3 Homogeneous Charge Compression Ignition Combustion .................................14

1.3.4 Genetic Algorithm Optimization in Engineering …….....................................15

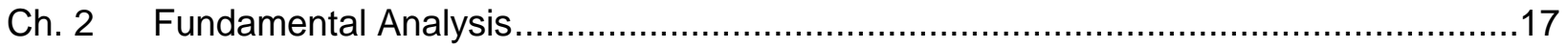

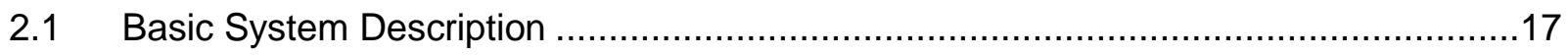

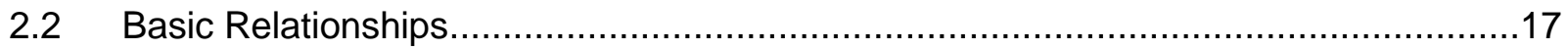

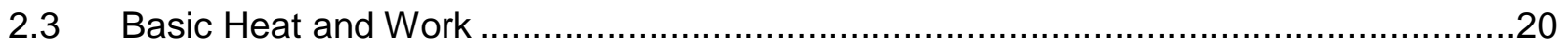

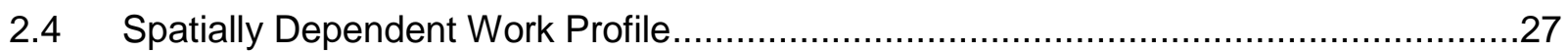

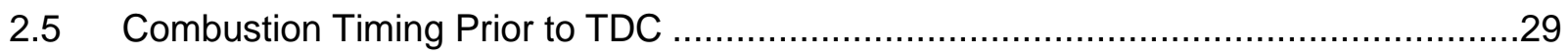

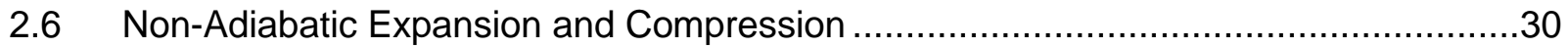

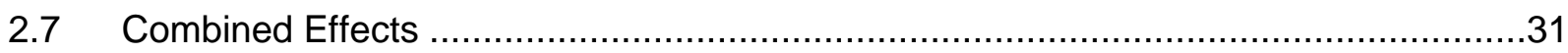

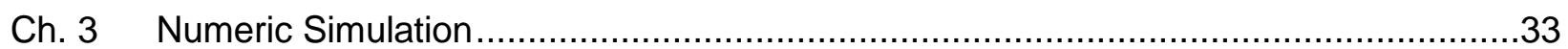

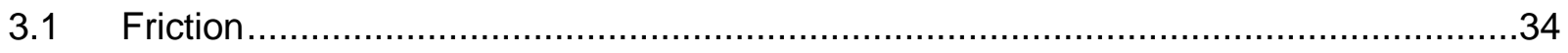

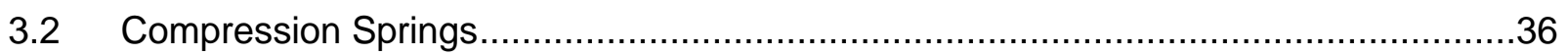

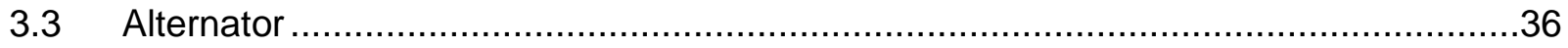

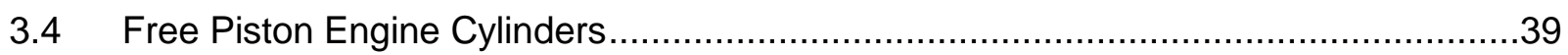

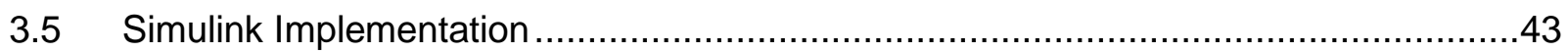

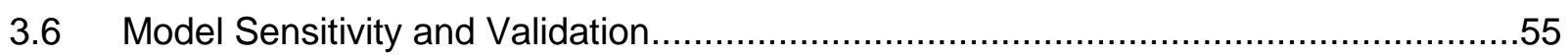

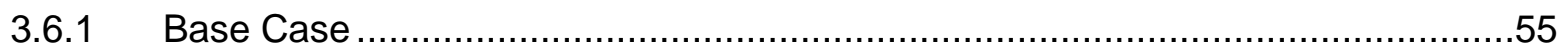

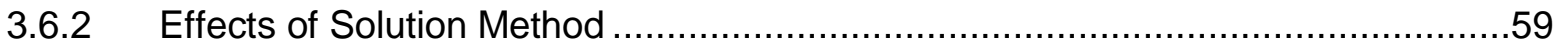

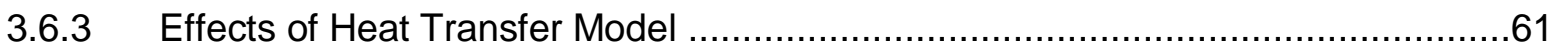

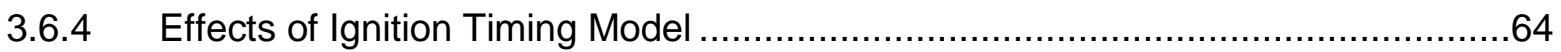

3.6.5 Effects of Combustion Heat Release Profile ................................................69 
3.6.6 Effects of Alternator Proportional Controller ..................................................73

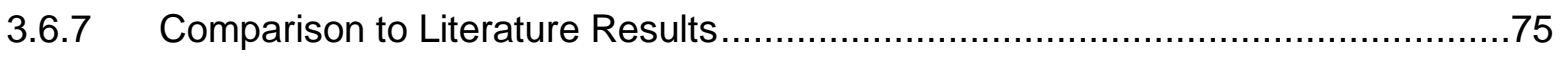

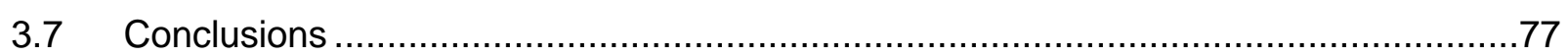

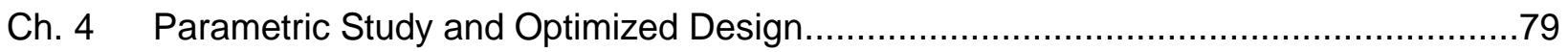

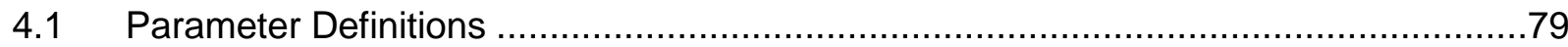

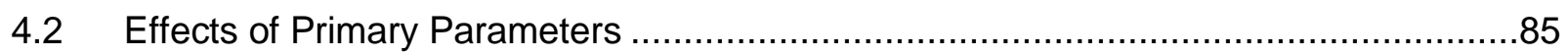

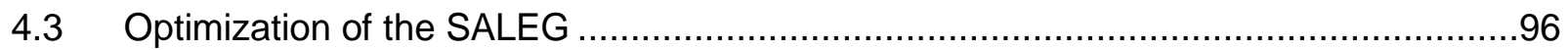

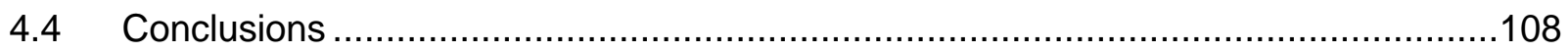

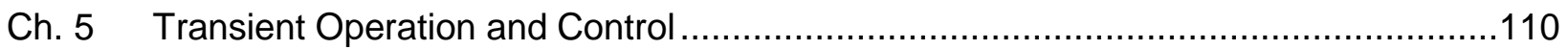

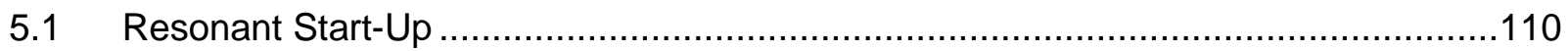

5.2 Response to Change in Load Demand ............................................................

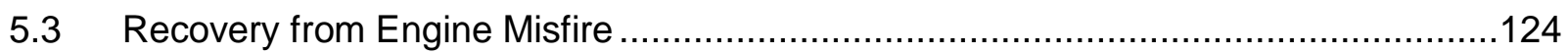

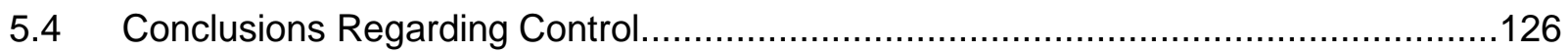

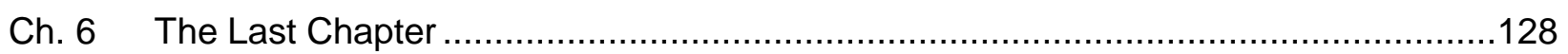

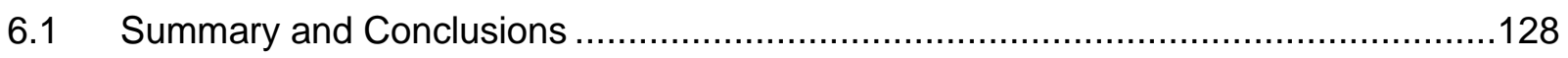

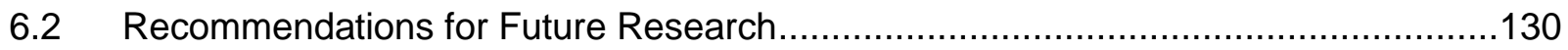

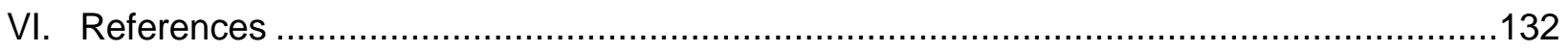

VII. Appendix A - Additional Simulink Model Diagrams ....................................................140

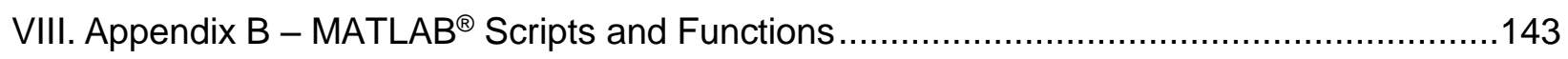

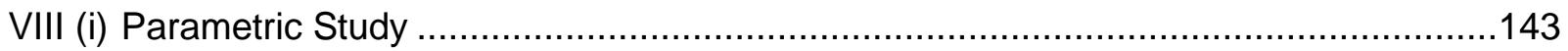

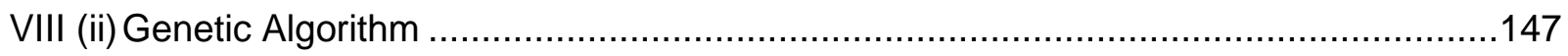

IX. Appendix C - Supplemental Figures from Parametric Study ......................................156 


\section{LIST OF FIGURES}

Figure 1: Illustration of dual free piston SALEG with selected dimensions included. ................ 2

Figure 2: Illustration of current SNL free piston engine device showing central combustion chamber and outlying pneumatic bounce chambers [15].

Figure 3: Early gasoline fueled, spark ignited, dual cylinder free piston engine experimental rig from WVU [16].

Figure 4: Schematic of Newcastle University's single cylinder free piston engine device with

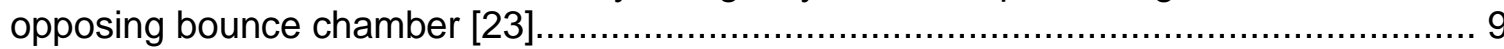

Figure 5: Illustration of dual free piston linear engine generator concept from WVU [62]..........13

Figure 6: Illustration of simplified dual free piston linear engine system for use in fundamental analysis.

Figure 7: Free body diagram of simple translator for fundamental analysis without friction or work terms.

Figure 8: Free body diagram of translator with resistive work force applied.

Figure 9: Basic free piston system including dimensionless heat and work [4]. Compression ratio rises as a result of energy imbalance.

Figure 10: Basic free piston system including dimensionless heat and work [4]. Compression ratio falls as a result of energy imbalance.

Figure 11: Basic free piston system including dimensionless heat and work [4]. Energy is balanced sufficiently for compression to rise to stability.

Figure 12: Basic free piston system including dimensionless heat and work [4]. Energy exchange is sufficiently balanced for compression to fall to stability.

Figure 13: Basic free piston system including dimensionless heat and work [4]. Energy is constrained to operate at equilibrium by the Otto cycle efficiency.

Figure 14: Constant work force profile compared to the cosine profile [5]. The integral of each profile over the position is constrained to be equivalent.

Figure 15: Comparison of the effects of constant and cosine work profiles on translator dynamics [5].

Figure 16: Fundamental simulations where the combustion pressure at $20 \%$ causes combustion to occur prior to top dead center [5].

Figure 17: Pressure curves to demonstrate heat transfer and adverse work [5]. The adiabatic profile is compared to the polytropic profiles. Advanced combustion timing and heat transfer combine to allow adverse work.

Figure 18: Dynamic profiles for multi-cycle simulation [5]. Fundamental analysis concepts are combined for a high compression equilibrium simulation. 
Figure 19: Transient settling of compression ratio and energy balance for multi-cycle simulation

[5] - continued from Figure 18. 32

Figure 20: Free body diagram of SALEG translator with forces and inertia terms included. ......34

Figure 21: Equivalent circuit for alternator modeling - adapted from [62] ...........................38

Figure 22: Master level of the Simulink model...........................................................44

Figure 23: 'Plant' subsystem of the Simulink model. .................................................45

Figure 24: 'Electrical output' subsystem of the Simulink model. .........................................45

Figure 25: 'Translator Dynamics' subsystem of the Simulink model. .................................46

Figure 26: 'Forces' subsystem of the Simulink model. ....................................................47

Figure 27: 'Alternator' subsystem of the Simulink model. ..............................................48

Figure 28: 'Friction' subsystem of the Simulink model. .................................................48

Figure 29: 'Springs' subsystem of the Simulink model...............................................48

Figure 30: 'Right_Cylinder' subsystem of the Simulink model. .........................................49

Figure 31: 'Cylinder States' subsystem of the Simulink model...........................................50

Figure 32: 'Cylinder model' subsystem of the Simulink model. .......................................51

Figure 33: ‘Compression' subsystem of the Simulink model..............................................52

Figure 34: 'Combustion' subsystem of the Simulink model..............................................53

Figure 35: '1D Pressure Wiebe HR' subsystem of the Simulink model. .................................54

Figure 36: Velocity versus position profile for the testing and validation base case................56

Figure 37: Acceleration versus velocity profile for the testing and validation base case. ..........57

Figure 38: Pressure versus position profile for the testing and validation base case. ..............57

Figure 39: Pressure and heat flux as functions of time for the testing and validation base case.

Figure 40: Power and efficiency as functions of time for the testing and validation base case...58

Figure 41: Energy balance for the testing and validation base case. .59

Figure 42: Example of the testing and validation base case achieving steady state after 1 second of virtual time.

Figure 43: Effects of Hohenberg parameters on frequency and heat flux. 62

Figure 44: Comparison of cylinder pressure and heat flux profiles four heat transfer models. ...63

Figure 45: Comparison of translator dynamic profiles four heat transfer models. .63

Figure 46: Effect of varying the CFR ignition model leading coefficient $(A)$ on pressure profile. 64 
Figure 47: Effect of varying the CFR ignition model temperature coefficient $(b)$ on translator dynamic profiles. The color of each profile corresponds to the pressure profiles in Figure 48.

Figure 48: Effect of varying the CFR ignition model temperature coefficient $(b)$ on pressure profiles. The color of each profile corresponds to the dynamic profiles in Figure 47.......66

Figure 49: Effect of equivalence ratio on CFR model ignition timing prediction on cylinder pressure. 66

Figure 50: Effects of varying CFR model ignition timing parameters on compression ratio, efficiency, frequency, and total heat transfer.

Figure 51: Comparison between CFR and RCM ignition timing models on cylinder pressure....68

Figure 52: Effects of EGR on the base case cylinder pressure with the RCM ignition model.....68

Figure 53: Effect of varied combustion duration on translator dynamic profiles. The color of each profile corresponds to those seen in Figure 54 ........................................................70

Figure 54: Effect of varied combustion duration on cylinder pressure. The color of each profile corresponds to those seen in Figure 53.

Figure 55: Effects of varied combustion duration on base case frequency, compression ratio, efficiency, and heat transfer. Stable and unstable cases are distinguished by ' 0 ' or ' $x$,' respectively.

Figure 56: Comparison between single and double Wiebe heat release profiles. .72

Figure 57: Effects of the varied burn profiles on cylinder pressure for the testing and validation base case.

Figure 58: Normalized steady state performance characteristics compared when the alternator proportional controller gain is varied.

Figure 59: Effects of target stroke length on translator dynamics. Steady state stroke length and velocity are shown to increase as target stroke length is raised...................................74

Figure 60: Comparison of simulation to University of Ulsan experimental data.........................76

Figure 61: Comparison of current simulation to West Virginia University experimental data......76

Figure 62: Illustration of the right half of the SALEG with primary dimensions included. .79

Figure 63: Alternator parameter map for a two pole design based on Eq. 60. Points are given for reference where $\mathrm{X}$ indicates stroke, $\mathrm{Y}$ indicates frequency, and $\mathrm{Z}$ indicates diameter. ..81

Figure 64: Illustration of the basic piston head design. The underside of the piston head is shown in the forefront and the crown of the piston head is assumed to be a flat surface.

Figure 65: Quadratic relationship for the mass of piston heads dependent on bore diameter....83

Figure 66: Comparison between natural frequency increase with and without the inclusion of spring inertia variation. 
Figure 67: Parametric sweep of bore showing its effects on compression ratio, stroke, and stability.

Figure 68: Parametric sweep of bore showing its effects on frequency, efficiency, power density, and peak pressure.

Figure 69: Parametric sweep of neutral clearance length showing its effects on compression ratio, stroke, and stability.

Figure 70: Parametric sweep of neutral clearance length showing its effects on frequency, efficiency, power density, and peak pressure.

Figure 71: Parametric sweep of relative port location showing its effects on stability, compression ratio, and stroke length.

Figure 72: Parametric sweep of relative port location showing its effects on frequency, efficiency, power density, and peak pressure.

Figure 73: Parametric sweep of intake pressure showing its effects on stability, compression ratio, and stroke length.

Figure 74: Parametric sweep of intake pressure showing its effects on frequency, efficiency, power density, and peak pressure.

Figure 75: Parametric sweep of intake temperature showing its effects on stability, compression ratio, and stroke length. .92

Figure 76: Parametric sweep of intake temperature showing its effects on frequency, efficiency, power density, and peak pressure.

Figure 77: Parametric sweep of spring stiffness showing its effects on stability, compression ratio, and stroke length.

Figure 78: Parametric sweep of spring stiffness showing its effects on frequency, efficiency, power density, and peak pressure.

Figure 79: Parametric sweep of equivalence ratio showing its effects on stability, compression ratio, and stroke length.

Figure 80: Parametric sweep of equivalence ratio showing its effects on frequency, efficiency, power density, and peak pressure.

Figure 81: Parametric sweep of EGR showing its effects on stability, compression ratio, and stroke length.

Figure 82: Parametric sweep of EGR showing its effects on frequency, efficiency, power density, and peak pressure.

Figure 83: Flow chart diagram for the genetic algorithm optimization routine.

Figure 84: Illustrations to describe mutation and crossover genetic alterations.

Figure 85: Typical weight function profiles for performance value calculation in the genetic algorithm optimization routine.

Figure 86: GA optimization response for $2 \mathrm{~kW}$ (left) and $4 \mathrm{~kW}$ (right) SALEG devices. 
Figure 87: Design map of maximum stroke to bore ratio, compression ratio, efficiency, and overall performance for the $4 \mathrm{~kW}$ SALEG. Lower limits are applied so that intake temperature is greater than $300 \mathrm{~K}$, intake pressure is greater than $1 \mathrm{bar}$, and equivalence ratio is greater than 0.35 for all data points.

Figure 88: Design map of maximum stroke to bore ratio, frequency, power density, and overall performance for the $4 \mathrm{~kW}$ SALEG. Lower limits are applied so that intake temperature is greater than $300 \mathrm{~K}$, intake pressure is greater than 1 bar, and equivalence ratio is greater than 0.35 for all data points. .104

Figure 89: Design map of equivalence ratio, intake temperature, compression ratio, and overall performance for the $4 \mathrm{~kW}$ SALEG. Lower limits are applied so that intake temperature is greater than $300 \mathrm{~K}$, intake pressure is greater than $1 \mathrm{bar}$, and equivalence ratio is greater than 0.35 for all data points. 104

Figure 90: Design map of equivalence ratio, efficiency, peak cylinder pressure, and overall performance for the $4 \mathrm{~kW}$ SALEG. Lower limits are applied so that intake temperature is greater than $300 \mathrm{~K}$, intake pressure is greater than 1 bar, and equivalence ratio is greater than 0.35 for all data points. 105

Figure 91: Design map of intake temperature, frequency, power, and overall performance for the $4 \mathrm{~kW}$ SALEG. Lower limits are applied so that intake temperature is greater than $300 \mathrm{~K}$, intake pressure is greater than $1 \mathrm{bar}$, and equivalence ratio is greater than 0.35 for all data points. 106

Figure 92: Translator dynamic profiles and pressure traces for the Ch. 5 base case. 111

Figure 93: Forces acting on the translator and steady state energy analysis of the Ch. 5 base case.

Figure 94: Simulation of base case being motored by the inverted alternator with sinusoidal force profile. Force frequency is $35 \mathrm{~Hz}$ and force amplitude is varied.

Figure 95: Simulation of base case being motored by the inverted alternator with sinusoidal force profile. Force amplitude is $66 \mathrm{~N}$ and force frequency is varied.

Figure 96: Motor controller subsystem in the Simulink model used for SALEG start-up. .115

Figure 97: Controller outputs and engine cylinder transient response during start-up of the SALEG.

Figure 98: Forces acting on the translator, compression ratio, and stroke length during SALEG start-up.

Figure 99: Variations in SALEG start-up response as initial motor frequency is varied.

Figure 100: Response of the SALEG as it reacts to a step decrease in power demand. Runaway compression results from using only the alternator $\mathrm{P}+\mathrm{PI}$ controller to respond to the change in load demand.

Figure 101: Parameter sweep of intake temperature showing the relationships between intake temperature, power, and compression ratio. 
Figure 102: Successful response of the SALEG to a step decrease in load demand. Target compression ratio is matched to power demand and intake temperature is controlled to meet target compression ratio.

Figure 103: Successful response of the SALEG to a ramp decrease in load demand. Target compression ratio is matched to power demand and intake temperature is controlled to meet target compression ratio.

Figure 104: Relationship between equivalence ratio, power, and compression ratio.

Figure 105: Intake temperature and equivalence ratio maps for power and compression ratio.

Figure 106: Simulated short duty cycle of SALEG with motored resonant start-up, steady state operation, and ending with fuel being cut.

Figure 107: Steadily increasing alternator load applied to the SALEG translator, eventually resulting in engine misfire. Rapid loss of compression follows the misfire and results in engine ring-down.

Figure 108: Transient response and recovery of SALEG stable operation after misfire via the resonant start-up controller.

Figure 109: 'Translator State' subsystem of the Simulink model. ......................................140

Figure 110: 'Cylinder Pressures' subsystem of the Simulink model. .................................140

Figure 111: 'Left_Cylinder' subsystem of the Simulink model..........................................141

Figure 112: 'Cylinder States' subsystem of the Simulink model.......................................142

Figure 113: 'Intake control' subsystem of the Simulink model. ........................................142

Figure 114: 'Alternator control' subsystem of the Simulink model....................................142

Figure 115: Effects of EGR on stability, compression ratio, and stroke length for the testing and validation base case with the RCM ignition delay model.......................................156

Figure 116: Effects of EGR on frequency, efficiency, power density, and peak pressure for the testing and validation base case with RCM ignition delay model .............................156

Figure 117: Effects of maximum stroke to bore ratio on performance, compression ratio, and stroke length. Performance is calculated based on the weight profiles given in Figure 85,

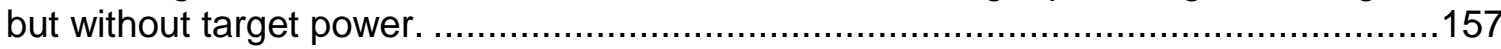

Figure 118: Effects of maximum stroke to bore ratio on frequency, efficiency, power density, and

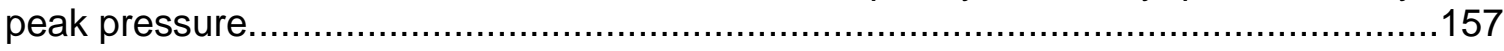

Figure 119: Effects of dimensionless intake pressure on performance, compression ratio, and stroke length. Performance is calculated based on the weight profiles given in Figure 85,

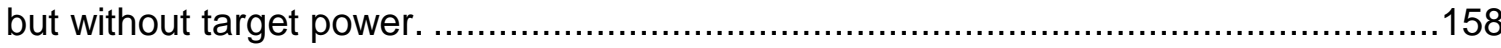

Figure 120: Effect of dimensionless intake pressure on frequency, efficiency, power density, and peak pressure. 158 
Figure 121: Effects of dimensionless heat addition on performance, compression ratio, and stroke length. Performance is calculated based on the weight profiles given in Figure 85, but without target power.

Figure 122: Effect of dimensionless heat addition on frequency, efficiency, power density, and peak pressure.

Figure 123: Effects of frequency ratio on performance, compression ratio, and stroke length. Performance is calculated based on the weight profiles given in Figure 85, but without target power.

Figure 124: Effects of frequency ratio on frequency, efficiency, power density, and peak pressure.

\section{LIST OF TABLES}

Table 1: Parameters and steady state performance characteristics for the base case used for testing and validation.

Table 2: Effects of step size on the steady state solution while all other base case attributes are held constant.

Table 3: Effects of Simulink solver order of accuracy on the steady state solution while all other base case attributes held constant. 60

Table 4: Effects of stop time on steady state simulation results............................................61

Table 5: Summary of effects for the base case simulation with varied burn profiles. …..............73

Table 6: Summary of prototype device parameters validated in literature................................75

Table 7: Summary of the dimensions and their descriptions from the SALEG illustration in Figure 62.

Table 8: Summary of independent parameters used to define the SALEG steady state design simulation.

Table 9: Summary of optimized SALEG devices based on weight maps in Figure 85. 101

Table 10: Summary of optimized SALEG devices with varied target power and tightened compression ratio constraint rangind from 5-25.

Table 11: Sensitivity of $4 \mathrm{~kW}$ optimized designs to variations of the GA performance weights.

Table 12: Final design optimization without target power included in performance evaluation.108

Table 13: Summary of design parameters and stead state performance characteristics of the

Ch. 5 base case. 


\section{NOMENCLATURE}

Symbol

ANN

BDC

$b$

CFD

CFR

$\mathrm{CO}$

$C_{p}$

$C_{v}$

DLR

EGR

EMF

FBD

$f, f_{\text {avg }}$

$F_{f}$

fmep

$F_{P}$

FPLE

$F_{\text {spring }}$ or $F_{S}$

$F_{\text {work }}$ or $F_{w}$

$\mathrm{GA}$

$\mathrm{HCCl}$

ICE

$k$

$L$

$L_{a}$

$L_{C}$

LEM

$L_{f l}$

LHV

$L_{P}$

$L_{p o}$
Description

Mathematical Terms and Abbreviations

translator instantaneous acceleration (Eq. 11)

artificial neural network

piston bottom dead center

engine bore (Eq. 1)

computational fluid dynamics

Cooperative Fuel Research

carbon monoxide

specific heat at constant pressure (Eq. 2)

$\mathrm{kJ} /(\mathrm{kg} \cdot \mathrm{K})$

specific heat at constant volume (Eq. 2)

German Aerospace Center

exhaust gas recirculation

electromotive force

free body diagram

engine frequency or average frequency (Eq. 37)

friction force on translator (Eq. 36)

friction mean effective pressure $\quad \mathrm{Pa}$

engine cylinder pressure force on translator (Eq. 4)

free piston linear engine

spring force on translator (Eq. 5)

$\mathrm{kJ} /(\mathrm{kg} \cdot \mathrm{K})$

work force on translator (Eq. 25)

genetic algorithm

homogeneous charge compression ignition

internal combustion engine

spring stiffness (Eq. 5)

$\mathrm{N} / \mathrm{m}$

midpoint engine chamber clearance length (Eq. 1)

half length of alternator moving mass (Figure 1)

cylinder length from midpoint to head (Eq. 1)

linear electric machine

free length of mechanical spring (Eq. 5)

lower heating value

piston length from translator midpoint to head (Eq. 1)

location of ports opening (Figure 1)
$\mathrm{Hz}$

$\mathrm{N}$

$\mathrm{m}$

$\mathrm{m}$

m

$\mathrm{m}$

$\mathrm{J} / \mathrm{kg}$

$\mathrm{m}$

$\mathrm{m} / \mathrm{s}^{2}$

$\mathrm{m}$

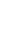$$
\mathrm{N}
$$$$
-
$$$$
\mathrm{N}
$$$$
\mathrm{N}
$$$$
-
$$$$
-
$$

m

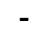

m

$\mathrm{m}$ 


\begin{tabular}{|c|c|c|}
\hline$m$ & translator mass (Eq. 6) & $\mathrm{kg}$ \\
\hline$m_{\text {gas }}$ & mass of cylinder gas (Eq. 16) & $\mathrm{kg}$ \\
\hline$n$ & polytropic exponent & - \\
\hline $\mathrm{NO}_{x}$ & Oxides of Nitrogen & - \\
\hline$P$ & engine cylinder pressure (Eq. 2) & $\mathrm{kPa}$ \\
\hline PID & proportional-integral-derivative & - \\
\hline$Q$ & heat (Eq. 16) & $\mathrm{kJ}$ \\
\hline$R$ & specific ideal gas constant (Eq. 18) & $\mathrm{kJ} /(\mathrm{kg} \cdot \mathrm{K})$ \\
\hline $\mathrm{RCM}$ & rapid compression machine & - \\
\hline RMS & root mean squared & dependent \\
\hline rpm & revolutions per minute & $\mathrm{rev} / \mathrm{min}$ \\
\hline SALEG & spring assisted, linear engine generator & - \\
\hline$S, S_{\max }$ & translator stroke or maximum stroke length (Eq. 37) & $\mathrm{m}$ \\
\hline SNL & Sandia National Laboratories & - \\
\hline SOC & start of combustion & - \\
\hline$T$ & temperature (Eq. 16) & $\mathrm{K}$ \\
\hline$t$ & time (Eq. 6) & s \\
\hline TDC & piston top dead center & - \\
\hline TPMLA & tubular permanent magnet linear alternator & - \\
\hline$v$ & translator instantaneous velocity (Eq. 12) & $\mathrm{m} / \mathrm{s}$ \\
\hline$V$ & engine cylinder volume (Eq. 1) & $\mathrm{m}^{3}$ \\
\hline$V_{d}$ & total engine displacement volume (Eq. 37) & $\mathrm{m}^{3}$ \\
\hline$W_{f}$ & friction work (Eq. 37) & $\mathrm{J}$ \\
\hline WVU & West Virginia University & - \\
\hline$x$ & translator instantaneous position, a function of time (Eq. 1) & $\mathrm{m}$ \\
\hline$x_{l}, x_{r}$ & left or right translator dead center position (Eq. 14) & $\mathrm{m}$ \\
\hline
\end{tabular}

\section{Subscripts and Superscripts}

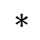

in

$l$ or $L$

$r$ or $R$

$m p$

$i$

1,2

ss indicating dimensionless term

indicates being added to a system

referring to the left

referring to the right

referring to the midpoint

referring to the current step in time or space

referring to the state (first or second)

referring to steady state operation 


\begin{tabular}{ccc}
\hline & Greek Variables & - \\
\hline$\gamma$ & ratio of specific heats (Eq. 3) & $\mathrm{m}$ \\
$\delta$ & spring deflection (Eq. 41) & - \\
$\eta_{\text {alt }}$ & alternator efficiency (Eq. 45) & -
\end{tabular}




\section{CH. 1 INTRODUCTION}

The worldwide energy market is facing many challenges. Among these is the sustainability of modern crankshaft driven engine technology. Current energy storage technology cannot support a widespread transition to electric vehicles [1], but tightening regulations against harmful emissions and greenhouse gases places great strain on engine manufacturers to meet consumer demands as well as regulatory requirements. While marginal improvements are still available in various forms (such as variable valve events, increased compression, higher pressure fuel injection, and sophisticated control), these technologies are expensive and may not represent the level of advancement necessary to meet the full spectrum of demands. Power transmission through a mechanical drivetrain adds frictional losses as well as complexity and cost to the system. The conversion from rotation of the crankshaft to translation of the piston results in bearing friction on the crankshaft and side thrust on the piston which increases ring friction and heat loss to the engine cylinder walls. Additionally, conventional engine technology suffers from losses as energy is mechanically transmitted to the valve train, and overall system efficiency is lessened further if peripheral components such as cooling, electrical, and aftertreatment are included. For the on-road diesel engine specifically, exhaust treatment has become an integral part of the overall strategy to meet emissions requirements [2]. Unfortunately, exhaust aftertreatment components also carry consequences for cost, efficiency, and power.

Hybrid electric vehicles are a leading solution to the challenge of transitioning away from conventional engine technology [3]. However, hybrid strategies, especially those that can meet the demands of long range commuter customers, must depend on the reliability and power density of conventional engine technology. For the sake of performance and cost, it is desirable to move away from the conventional slider-crank engine towards a technology that can meet power demands while reducing weight, cost, and complexity and raising efficiency and flexibility.

Compared to conventional crankshaft engine technology, the proposed spring assisted, linear engine generator (SALEG) has the potential to operate with a reduced number of moving parts, increased power density, and higher efficiency. Furthermore, the SALEG directly produces electrical power which can be efficiently used or stored for potential use in a hybrid electric powertrain solution. Other potential applications for the SALEG are further explored in the literature review.

The focus for this investigation is aimed at the development of a steady state generator system which is optimized for efficiency to meet target power demand while constrained by given operating conditions. The research supports the overall goal of a commercially viable free piston linear engine generator that can effectively support transient operational demands. Beginning with implementation of 
the SALEG in a steady state application helps to build foundational understanding that can support its later deployment in more demanding environments such as the hybrid electric vehicle.

\subsection{SALEG Description}

The spring-assisted linear engine generator depends on only a single moving part: the free piston translator. Figure 1 illustrates the SALEG with the free piston translator separated for clarity. The translator includes the two piston heads, a connecting rod, and the permanent magnets. The translator oscillates within a housing that includes the engine compression cylinders and alternator stator windings. Driving the oscillations are opposed, two-stroke combustion cycles where the combustion and subsequent expansion of one cylinder drives the compression and subsequent combustion of the other.

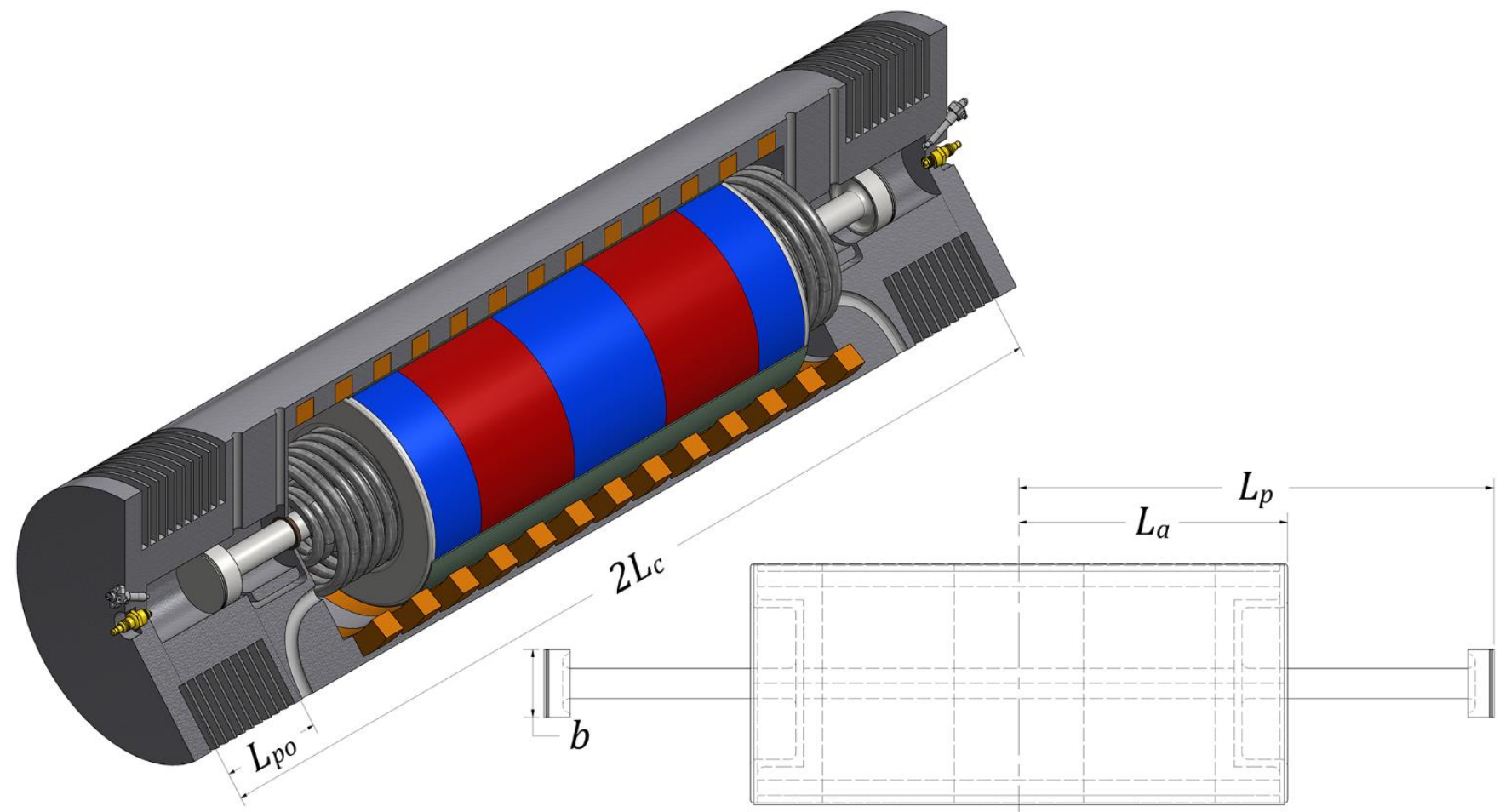

Figure 1: Illustration of dual free piston SALEG with selected dimensions included.

Dimensions in Figure 1 correspond to primary geometric parameters and include half the length of the alternator mover $\left(L_{a}\right)$, length from the translator midpoint to the piston crown $\left(L_{p}\right)$, length from housing midpoint to cylinder head $\left(L_{c}\right)$, length from cylinder head to port location $\left(L_{p o}\right)$, and piston bore (b). The illustration also includes fuel injectors and spark plugs at the heads of each cylinder. However, this investigation focuses on the marriage of the SALEG with Homogeneously Charged Compression Ignition $(\mathrm{HCCl})$ combustion - an autoignition combustion mode. The inclusion of a spark or glow plug may only be necessary for start-up or transient operation, and fuel injection may be accomplished by either direct or port injection schemes. Intake and exhaust events are accomplished by either electromechanical valves, allowing for quick and precise valve events, or by ports which are opened or 
closed as the piston translates within the cylinder space. In general, the two stroke $\mathrm{HCCl}$ engine involves fuel addition with the gas exchange process so that the fuel and air are well mixed prior to the start of combustion.

Electrical power is produced by an integrated tubular permanent magnet linear alternator. The poles of the alternator are securely mounted to the axially oscillating rod that connects the two oppositely facing pistons. Together, the rod, pistons, and alternator mover are called the translator. The motion of the translator is driven by the two stroke $\mathrm{HCCl}$ cycles and supported by mechanical or pneumatic springs located within the central chamber of the cylinder housing. The spring mechanisms act to provide potential energy storage which serves to buffer the system against adverse occurrences such as misfire or sudden load changes. The stiffness profile of the springs can be used to affect the frequency dynamics of the translator - varying the stiffness for a desired frequency regime.

In the free piston SALEG, the stroke length of the translator and compression ratio are not geometrically constrained as in a crankshaft driven engine. Instead, they are allowed to vary from stroke to stroke. This variability, while presenting difficulty in terms of system control, allows for a unique marriage between cycle to cycle flexibility and the $\mathrm{HCCl}$ combustion mode. Cyclic variations are sympathetic with the freedom of the translator to naturally increase or decrease its stroke length which acts as feedback for subsequent cycles of operation.

To investigate the SALEG, fundamental and numerical models have been built to represent the interactions of the thermodynamic and dynamic energy exchanges. The models are based on the application of Newton's second law. The cylinder is grounded and the translator oscillates within it according to forces from the engine cylinders, springs, friction, and integrated alternator system. These forces are represented by mathematical models which depend on known physical relationships, empirical constants, and instantaneous state values of the system. The fundamental model is used for basic understanding of the system. The numeric model is then employed for extended parametric, optimization, and transient analysis of the SALEG.

\subsection{Research Objectives}

Given the potential of the proposed free piston linear engine system to exceed conventional engine technology in efficiency, cost, and power density, there is a need to advance the state of the device. This investigation explores the design space available for the device, revealing trends and relationships, and advances the understanding of controller needs for robust support of the device during transient operation. 


\subsubsection{Objective 1: Fundamental and Numerical Simulations}

The primary objective of this research is to advance the understanding of the SALEG steady state operation, design space, and transient behavior. To facilitate the research, fundamental and numeric models have been constructed. The fundamental analysis takes advantage of various simplifications to achieve a mathematically closed form solution to represent the dynamic system. Then, a numerical simulation is built in MATLAB ${ }^{\circledR}$ and Simulink. Sensitivity analyses are conducted to demonstrate the significance of various assumptions within the model, especially empirical model parameters. Because experimental data is limited, validation is sought in three ways. Sensitivity analysis of the numerical methodology demonstrates the precision of the solution achieved by the numeric model; qualitative comparison between model prediction and results found in the literature shows the quality of accuracy of the numeric model; and, relationships between input parameters and system performance are verified against expected trends.

Both fundamental and numerical modeling efforts combine the dynamics of the mass-springdamper system with the electromechanical load from the generator and the thermodynamic processes within each cylinder. For the numerical model, special attention has been given to modularity so that the model can be extended to incorporate various methods for any of the involved processes. For instance, Hohenberg's heat transfer model is exchanged for Annand's as part of the sensitivity study. In general, each of the sub-models, including the engine cylinder models, are sufficiently sophisticated to represent the dynamics of the subsystem, but simple enough to allow for multi-cycle simulations of the system. These modeling efforts provide a foundational platform for the research objectives discussed in this dissertation as well as future investigations of free piston engine devices.

Stemming from the construction and analysis of the fundamental and numerical models, the first objective is to advance the fundamental understanding of free piston linear engines. This is achieved by assessing the mathematical tools used within the model to represent cylinder processes and system dynamics. Variations of heat transfer, combustion, and alternator force relationships found in the literature are compared, and their effects on system performance are evaluated.

Through this exercise, common numerical modeling tools are assessed with respect to their effects on SALEG behavior. The assessment advances the understanding of free piston engine generator modeling techniques and leads to insights regarding the impact of energy balance and the combustion process on steady state SALEG performance.

\subsubsection{Objective 2: Parametric Study and Optimization}

The numerical modeling in Objective 1 is extended to include integrated component design for the SALEG. Specifically, relationships for translator mass and device geometry are included in the 
simulation loop. This steady state design simulation is then used for parametric sweeps of input parameters and within an optimization routine to study the design space. To reduce the computation time, the complex Simulink model is compiled using the available rapid simulation target routine which converts the Simulink block sets to a $\mathrm{C}$ code executable program.

From the component design analysis, a total of eight independent input parameters are identified ranging from system geometry to engine operating conditions. The goal of the parametric study is to gain important insight into the design space of the SALEG regarding the effects of each design element on steady state performance. Each of the input parameters are studied with respect to their effects on energy balance and the consequences on compression ratio, stroke length, frequency, power density, efficiency, and stability. These discoveries aid in the discussion of system dynamical interdependence and lay the groundwork for the SALEG optimization study.

With multiple input parameters and output targets, the multi-dimensional design hyperspace is too vast for a basic optimization routine. Thus, a genetic algorithm (GA) is employed to explore the space effectively. The genetic algorithm combines widespread search of the design space (avoiding local maxima) with the ability to exploit the strengths of known solutions (akin to common gradient based optimization methods). Steady state targets and constraints are combined to quantify the performance value of a design solution. Based on varied design targets, multiple optimization points are provided. The primary goal of this objective is to advance the understanding of the design space available to the SALEG.

\subsubsection{Objective 3: Control for Start-up and Transient Response}

The third objective is to advance the understanding of start-up and transient responses of the SALEG. Simulink is chosen as the platform on which to develop the numerical model because of its modularity and robust functionality. The model is partitioned between the plant (where the system modeling is accomplished) and the controller. Controller architecture may take many forms, but control of the free piston and combustion events is paramount to the success of the SALEG. Initially, a standard proportional-integral $(\mathrm{PI})$ controller is employed to drive the system towards stability at target operation. The goal of this objective is to demonstrate control mechanisms capable of starting the SALEG and responding to adverse occurrences.

Start-up of the SALEG is achieved by inverting the alternator so that it applies a force and compels the motion of the translator. By matching the switching frequency of the motor to the natural resonance of the non-firing SALEG, sufficient compression can be reached for fuel injection and combustion. The alternator inverter is then immediately switched off so that energy is extracted from the system, preventing over-compression. The dynamically controlled start-up routine is demonstrated with respect to response times and steady state performance for a range of controller parameters. 
Responses of the SALEG to changes in load demand and misfire are demonstrated along with the ability of proportional and integral controllers to react effectively. Of greatest significance is fueling, intake conditions, and alternator loading. While some authority is available to quickly affect the alternator load, rapid changes in power demand can result in misfire or runaway compression. Fueling and intake conditions must be altered to compensate against such events. Map-based control architecture is demonstrated to achieve the desired responses.

\subsection{Review of Literature}

This literature review addresses both linear engine and alternator designs and behavior, as well as modeling and simulation techniques that can be used to aid in the optimization of a linear engine and alternator design. In addition, investigations of the free piston linear engine from major field researchers are presented. The fundamental analysis, previously published by the current author in two parts [4, 5], is presented in Ch. 2 of this dissertation, and it is expected that supplementary publications (e.g. [6, 7]) will result from research stemming

\subsubsection{Free Piston Linear Engines}

The reported efforts to advance development of the spring assisted linear engine generator are well grounded in the literature with a wide variety of prior art existing in the areas of both single and two cylinder devices. Much of the early development surrounds the use of the free piston in compressor devices. This application of free piston technology over the years, as well as three others, is summarized in a comprehensive timeline provided by Aichlmayr in [8] with more details reviewed by Mikalsen and Roskilly in [9]. Within the last two decades, the research has moved to emphasize electrical generation and hydraulic pumping. The concept of reduced mechanical losses and increased simplicity has attracted many researchers and innovators truly beginning in the mid-1990s. One of these examples is the 1995 US Army funded project granted to the Southwest Research Institute and University of Texas at Austin to explore the feasibility of the free piston linear engine alternator device as an auxiliary power device for hybrid electric vehicles [10].

A short time later (1998), work at Sandia National Laboratories (SNL) and West Virginia University (WVU) began in earnest. Investigations at SNL examined the use of $\mathrm{HCCl}$ combustion within a free piston engine through the use of a rapid compression machine which achieved Otto cycle, nearly constant volume combustion [11]. From this, they explored the idea of $\mathrm{HCCl}$ combustion within a free piston linear engine alternator through numeric modeling of the system [12]. Their work shows the potential of such a device and the importance of the cylinder recharging. Continuing in this vein, researchers at SNL extended their modeling towards optimization of scavenging and the alternator to maximize thermal efficiency [13]. They found that cycle-to-cycle variations in compression did not greatly affect scavenging 
performance and that steady, low temperature charge produced best results [14]. Today, development of their device continues under the sponsorship of the Department of Energy's (DOE) Vehicle Technologies Office in the form of a twin, opposed piston arrangement, which includes a single, centrally located combustion chamber and outlying pneumatic bounce chambers (analogous to mechanical springs in the proposed design) to aid in control. From this program, valuable data is being gathered from both modeling and prototype efforts [15].

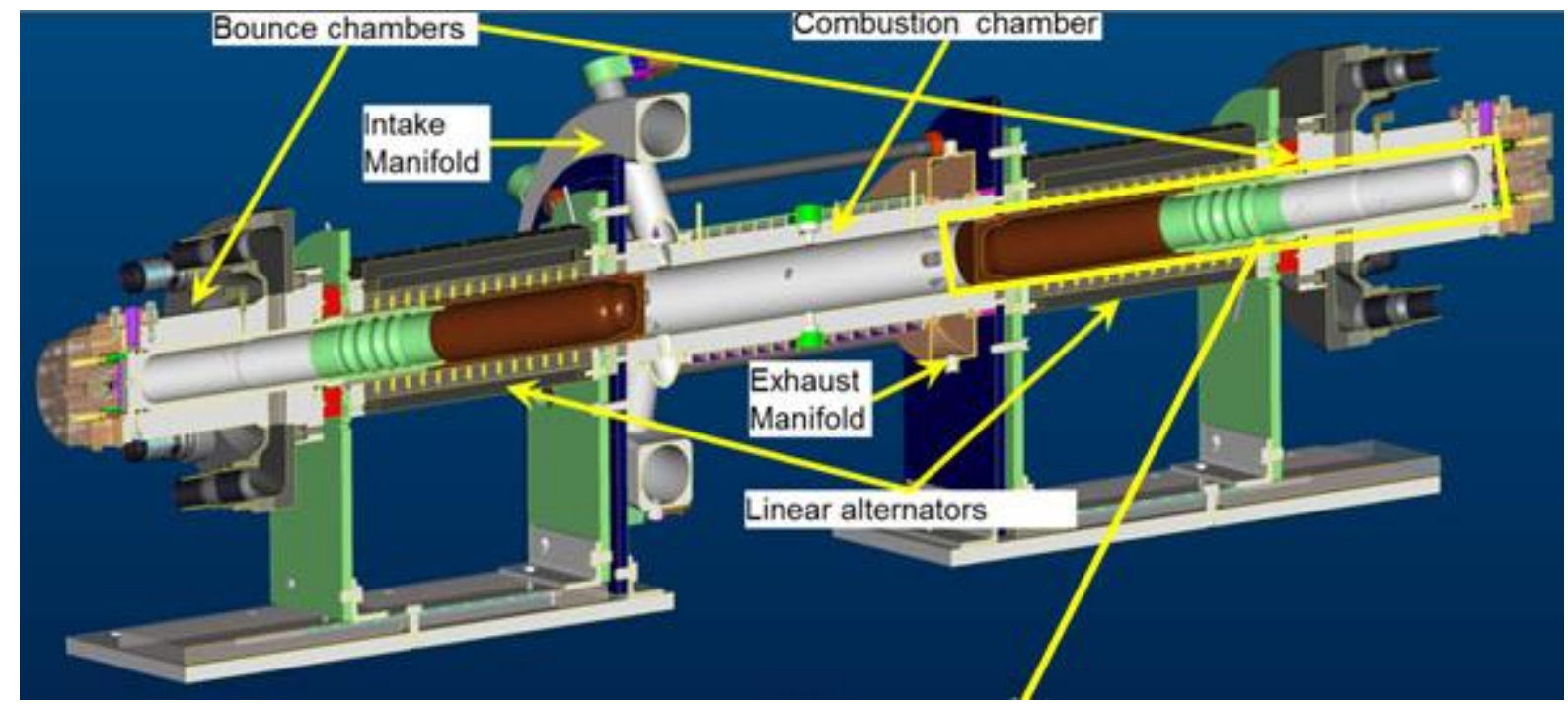

Figure 2: Illustration of current SNL free piston engine device showing central combustion chamber and outlying pneumatic bounce chambers [15].

Work on a dual cylinder device at WVU began with a parallel approach where an ad-hoc, off the shelf benchtop prototype model was built for experimentation while at the same time, a numeric investigation was conducted for verification and experimental simulation $[16,17]$. Valuable insight was gained from these works, particularly with regard to understanding adverse work around TDC and the implications of cycle to cycle energy balance for either stall or runaway compression ratio rise. This initial gasoline fueled prototype output $309 \mathrm{~W}$ over a sustained period of operation during which it was seen that stability depended greatly on combustion timing. The simulation provided the basis for a parametric study of the device and helped to close the loop in understanding the interactions between heat addition, combustion duration, peak pressure, engine frequency, and compression ratio. The effects of translator mass were also explored to show proportional relationships to stroke and compression and an inverse relationship to engine frequency. An image of the experimental rig is shown in Figure 3. 


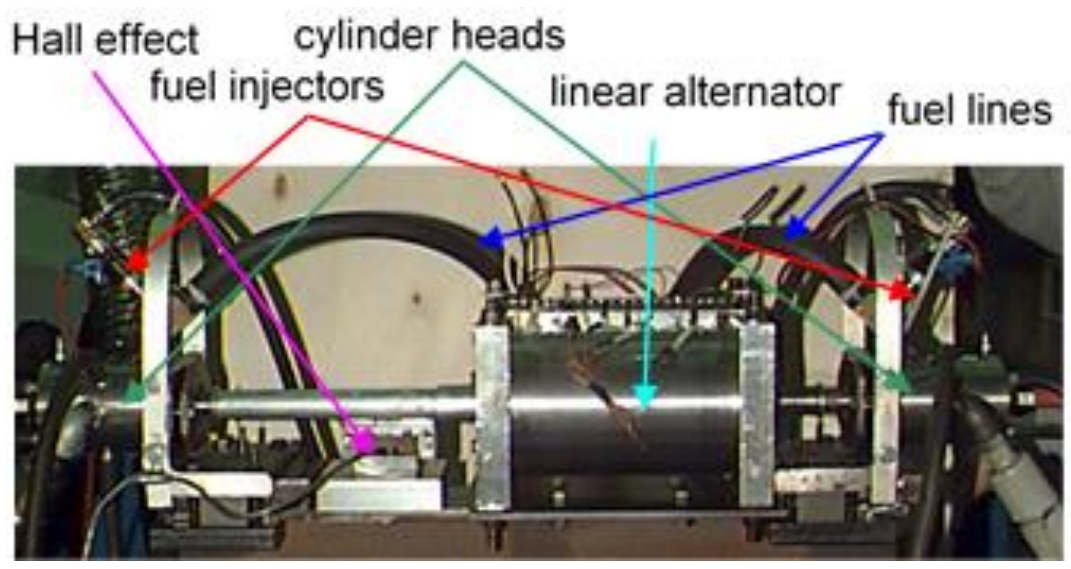

Figure 3: Early gasoline fueled, spark ignited, dual cylinder free piston engine experimental rig from WVU [16].

A second round of linear engine development at WVU applied simulation and artificial intelligence techniques to gain broader understanding of the effects of fuel/air ratio, moving mass, frictional losses, injection timing, and geometry on frequency and efficiency [18]. This fed the development of a second generation diesel fueled prototype. In this, a boosted, direct injected, port scavenged, dual cylinder engine operated between 50 and $60 \mathrm{~Hz}$ [19]. These research efforts revealed many important insights into the operation of the linear engine. Among these is the need for increased engine speed, giving rise to the implementation of springs presented in this dissertation as well as the reduction of translator mass for optimization.

Investigation of the free piston linear engine has continued since then. In 2004, the history and current state of the art in linear engine technology was reviewed [20], and more recently in 2011, operational data from the diesel prototype were analyzed and presented [21]. This work corroborated the findings from the first generation prototype, such as the mass relationship to frequency, and showed that raising the amount of fuel and advancing the injection timing both increased power output, efficiency, and frequency suggesting that the engine operated near the $\mathrm{HCCl}$ combustion regime. The fundamental analysis of the dual piston linear engine was published by the current author in 2014 [4, 5] and is described more fully in Ch. 2. The fundamental analysis helps to lay the foundation for this dissertation as well as the recently announced effort to build a spring assisted, single cylinder linear engine generator prototype [22].

Researchers at Newcastle University have been exploring the free piston linear engine device with a single combustion chamber opposed by a pneumatic bounce chamber (analogous to the proposed mechanical springs). Their endeavors in simulation were preceded by the extensive review of free piston technology, already mentioned [9], which concluded the most difficult challenge remaining for free piston devices is the control of the piston and that the free piston engine offers a viable platform for $\mathrm{HCCl}$ combustion. Since then, they have studied a variety of operating conditions including spark ignition [23] 
and compression ignition [24, 25]. Their work continues as they pursue the control of the device through various approaches including tailoring the bounce chamber pressure profile to the dynamics of the translator [26, 27]. A schematic of their device is presented in Figure 4.

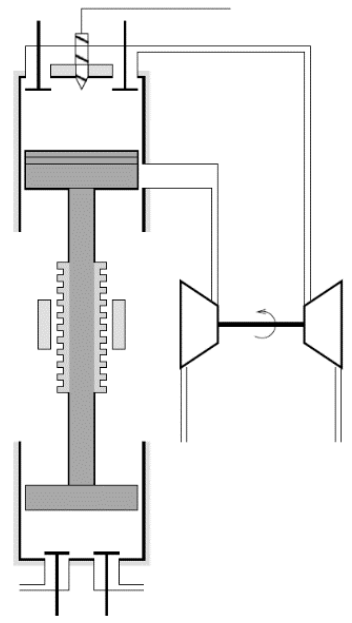

Figure 4: Schematic of Newcastle University's single cylinder free piston engine device with opposing bounce chamber [23].

The researchers at Newcastle have also recently published a review of the latest free piston publications to gain insights into current technical challenges and commercial expectations [28]. Cited challenges including piston motion, heat transfer, vibration, scavenging, and lubrication are impeding the viability of the free piston engine, and despite its known potential, there are few operational prototypes and no clear path to commercialization.

Researchers at the Beijing Institute of Technology published one of their earliest investigative efforts of the free piston engine in 2008 [29]. They report of their use of single free piston engine as a hydraulic pump in which the engine piston is directly coupled to a pumping piston. The combustion event compels the motion of the translator, and the hydraulic work fluid is compressed. The high pressure hydraulic rail is then used as supply for a bounce chamber which returns the translator for engine compression and cycle completion. The researchers utilized MATLAB ${ }^{\circledR} /$ Simulink tools to model the dynamic system response to various geometric parameters and hydraulic loading based on differential and empirical relationships. The numeric analysis was followed by experimental testing in 2010 [30] which discussed the relationships between compression pressure, injection timing, and combustion process and free piston diesel engine performance. Since then their attention has shifted to the application of the FPLE with a linear alternator. Zero dimensional modeling of a dual piston, spark ignition device was presented in [31] wherein a study of nondimensional parameters was used to set up design guidelines for the dual FPLE with alternator. From their work, efficiency is significantly dependent on ignition timing and there is an optimum timing that avoids adverse work during the compression stroke and reduced 
peak pressure from late combustion. Their numeric modeling work also includes dynamic simulations for the control of single cylinder FPLE generator which demonstrates the use of fueling to achieve steady state operation in response to a step change of the alternator load [32]. In general, use of the controller achieves stable oscillation with a new stable condition in around three cycles for the system operating around $56 \mathrm{~Hz}$.

In 2014, development continued with experimental testing of the start-up sequence which used the alternator as a motor to compel translator motion and increase compression [33]. Simulation results were compared with experimental data to show the accuracy of the simplified numeric model as good for the first four cycles and somewhat divergent thereafter. While this represents some validation for many of the numeric modeling techniques applied throughout the literature (including the current work), the report is also important because it announces the collaborative efforts between researchers at Newcastle and Beijing. Since then, the combined team has made significant contributions to FPLE development including further validation of the dual free piston, spark ignited model [34] and the simulation with experimental validation of a single cylinder, spark ignited FPLE generator opposed by a permanent magnet linear motor [35]. For the latter, the experimental device reached an output of $25.9 \mathrm{~W}$ at a system efficiency of $13.5 \%$. Finally, resonant start-up analyses demonstrate the relationships between bore, stroke, translator mass, and steady state frequency of a dual cylinder, diesel compression FPLE device in [36].

A team from the German Aerospace Center (DLR) is working towards a single piston, $8 \mathrm{~kW}$ prototype which includes a mechanical spring for rebounding and operates around $20 \mathrm{~Hz}$ within a battery backup hybrid electric vehicle. Beginning in 2012, the team at DLR started with individual component testing by using controlled hydraulics to emulate the motion of a free piston engine [37]. A two stroke, gasoline fueled, head scavenged engine system is modeled and tested in [38], and the linear electric generator is investigated for optimization in [39, 40]. Today, a functional demonstrator has provided up to $10 \mathrm{kWe}$ at a frequency of approximately $20 \mathrm{~Hz}$, and one of the major challenges remains to be robust control of the free piston. Just as in the prototype development at WVU, slow translator speed is associated with high linear alternator moving mass (the DLR alternator provides a maximum force of 12 $\mathrm{kN})$. The DLR prototype demonstrates a total efficiency of $17.9 \%$ with $5.9 \%$ fuel energy lost to friction and $68.2 \%$ lost to combustion inefficiency [41].

In addition to these more expansive bodies of work is a number of interesting patents and research pieces aimed at developing the linear engine concept in various forms. While a significant review of commercial developments is provided by Newcastle researchers in [28], some of the other research works are provided here. Pempek Systems (Australia) developed the FP3 concept device which fitted three free piston linear engines into a package [42]. Researchers at Chalmers University of Technology 
simulated a dual free piston device to investigate its multifuel capabilities [43]. Researchers from IFP, France undertook simulation work which showed the use of high compression and EGR can yield linear engine indicated efficiencies around 50\% [44]. Toyota has officially announced and is currently working towards a single cylinder, $10 \mathrm{~kW}$, bounce chamber supported device for use in a hybrid electric vehicle system $[45,46]$. Investigators from South Korea's Ulsan University has an operational prototype and has extensively modeled a spring supported, propane fueled, spark ignited, dual cylinder free piston linear engine generator to explore the control of the device and possibilities for transitioning from spark to $\mathrm{HCCl}$ operation [47]. Results from their prototype device are presented in [48] demonstrating a range of operating conditions and performance outcomes. Perhaps most relevant to this dissertation is their experimental analysis of the spring stiffness effects on combustion and free piston translator dynamics [49]. The novel arrangement of their dual piston device makes use of four equivalent compression springs. Reported are five cases with spring stiffness ranging from $0 \mathrm{kN} / \mathrm{m}$ to $14.7 \mathrm{kN} / \mathrm{m}$. Experiments show that raising the spring stiffness results in increased translator frequency (raised from $35 \mathrm{~Hz}$ to 47 $\mathrm{Hz}$ ), increased thermal efficiency (from $6 \%$ to $7.22 \%$ ), and increased power output (on the order of $25 \%$ power increase depending on operating conditions).

From the reviewed literature, it is clear that the proposed implementation of springs to raise frequency and augment the performance of a free piston linear engine is well supported but in need of refinement. Further, it is believed that the springs (or spring-equivalent devices) offer the dual benefit of tailoring the piston dynamics for $\mathrm{HCCl}$ operation. In the next chapter, the influence of springs within an engine system is explored at a fundamental level - a summary of the author's published works $[4,5]$.

\subsubsection{Linear Electric Machine}

The SALEG produces power by directly converting the linear motion of the translator to electrical current through the use of a tubular permanent magnet linear alternator. The linear alternator is an example of a linear electric machine (LEM). These take on two basic applications which are defined essentially by the direction of power flow: into or out of the system. The first, which gained much focus in early research, is the linear motor, which has already been mentioned as the inverted linear alternator being used for start-up of the SALEG. The second, the linear generator or alternator, has become increasingly attractive over the past couple decades. Multiple forms of the LEM exist ranging from the tubular permanent magnet device used in the current research to one- and two-sided, flat-type linear inductance machines, and it is often dependent on the engineering application as to which is best suited. Regardless of the application though, the fundamental theory of the linear electric machine draws from the same set of principles. 
One of the earliest presentations of the theory as applied to permanent magnet linear machines was made by Boldea and Nasar in [50] which described the differential equations for single- and threephase machines. This first paper focused on the theory, and their next demonstrated the physical design of a tubular type linear generator machine based on theory and practical engineering application [51]. The design steps outlined in the second work are widely cited and used to define geometric parameters for the machine including the magnets and stator windings. Later, Nasar and Chen applied a minimizing optimization routine to design such a device based on machine specifications and related constraints [52].

In modeling the linear machine, a variety of approaches have been taken. One of the earliest found in literature is that mentioned above which developed fundamental relationships to describe a permanent magnet system. The difficulty faced is the ability of the fundamental equations to capture the true physical nature of the oscillating component. While useful to describe the device at a high level, idealizations and simplifications were applied that undermined the general accuracy. Other methods both simple and complex - have been applied to the problem. One such is the use of an equivalent electrical circuit.

The equivalent circuit method was introduced for a linear machine as early as 1987 by Nasar and Chen [53]. Here they presented the analysis and development of a circuit which was able to account for saturation, leakage, and armature reaction of a tubular permanent magnet linear alternator (TPMLA). In 1988, Pai and Boldea introduced an equivalent circuit for a linear induction motor [54]. Results of their per-phase circuit model were then compared and validated against experimental testing. In these and other examples which present equivalent circuits, component parameters (inductance and resistance values) typically depend on either results obtained from experimentation or from field analysis of a defined device. This leads to the next commonly employed modeling tool: finite element analysis.

One of the earliest finite element method (FEM) approaches for modeling a linear machine is seen in [55] which presents the use of both field analysis and FEM to develop machine design and equivalent circuit parameters for a linear induction machine. Their work was shown to achieve sufficient accuracy, and FEM is now a commonly employed tool for LEM development.

Modern computational power combined with these modeling techniques allows for sophisticated simulations of linear machines. Consequentially, understanding operational characteristics and evaluating performance of a machine is within reach at an early development stage. Furthermore, parametric studies provide sound justification for design choices. In 2012, the use of 3-D FEM to analyze and predict the performance of a three-phase, tubular transverse flux, permanent magnet machine was demonstrated [56]. The FEM analysis was applied to study flux distribution, density, leakage, harmonics, 
EMF, cogging force, and main thrust force, and comparison with experimental results showed good agreement. In 2015, a magnetic equivalent circuit was employed to model and size a TPMLA [57]. The resulting position dependent circuits were validated against FEM solutions for the device and the cancelation of end effects was achieved by extending the stator with appropriately sized teeth. Lu and Ye employed FEM for the design and analysis of a TPMLA in 2009 [58]. In the analyzed device, a large air gap separation is required and a copper layer is applied to directly produce eddy current power. The dynamic FEM analysis was used to compare geometric parameters and radially versus transversely magnetized structures for optimal performance. Radial is shown to achieve higher power density and results are validated against prototype operation. This is a small sample of the many examples of LEM modeling that can be found in the literature.

Linear electric machines are of interest for many applications where linear motion is involved. In some cases, the importance is actuation by the LEM. In others, power generation through the LEM is achieved as the translating component is forced through the LEM stator component. In generating applications, translator motion can be forced in various ways such as the periodic motion of tidal waves or combustion pressure. Focusing on the use of linear electric alternators within combustion engines, a few important works are described next.

The earliest developments by Nasar and Boldea concerned the use of Stirling free piston engines. More recently, French researchers presented the optimization of a linear induction generator mover [5961]. A detailed theoretical analysis was first provided and then followed by a global simulation of the device, including its control. Then, multi-objective optimization is performed to identify three solutions from the cost-per-unit-of-power design space. Similar to the free piston Stirling application, the free piston internal combustion engine has been gaining momentum for the past 20 years as a viable application for LEM's.

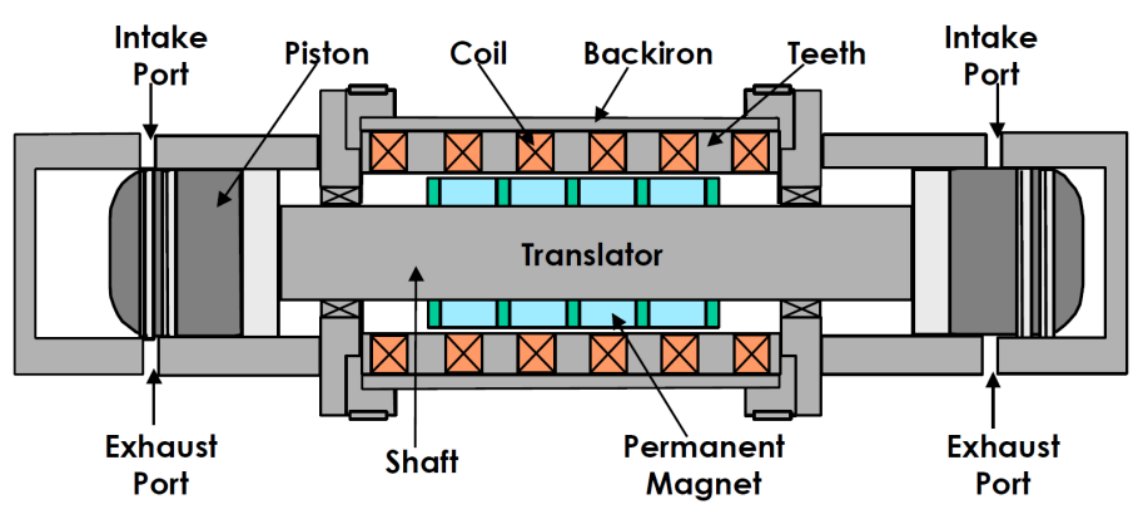

Figure 5: Illustration of dual free piston linear engine generator concept from WVU [62].

Cawthorne of WVU presented a focused dissertation on the optimized design of a TPMLA [62]. With simplified engine modeling for the dual free piston components (see Figure 5 for illustration), FEM 
analysis, and robust MATLAB ${ }^{\circledR}$ routines, a detailed iteration scheme was constructed to design and model the performance of the LEM. This was followed by optimization which found design parameters based on air or iron core topology to maximize efficiency and minimize translating mass. Many of the research groups previously mentioned as developing the free piston linear machine are assuming the use of a TPMLA for high efficiency energy conversion. One of the leading developers of the linear alternator, Libertine FPE Ltd. ${ }^{\circledR}$ from the UK, is currently in the prototype testing phase.

Problems for the linear electric alternator become evident upon examination of the literature. Development efforts are being put into reduction of cogging and detent forces. Cogging forces are seen as the high frequency ripples evident in the force profile and are related to the geometric arrangement of the stator windings and translating magnets while detent forces include the end effects which vary with the relative length of the moving component. As the translator moves through its stroke, these fluctuations of the force profile can have adverse effects on translator dynamics. Methods of reducing their effects are given in $[63,64]$ and amount to optimized component design. Control of the linear electric machine is also a concern though often closely tied to the control of the device to which it is married. Finally, device optimization is difficult, and numerous optimization studies can be found in the literature for a wide set of applications. Balancing the mass, speed, control needs, force and power requirements, price, and other performance characteristics is difficult for any complex system and no exception for the linear electric machine.

\subsubsection{Homogeneous Charge Compression Ignition Combustion}

The simplest description of a $\mathrm{HCCl}$ combustion event involves a quasi-homogeneously mixed chamber of fuel and air which is compressed until ignition occurs. Due to the homogeneity of the mixture, ignition occurs at many points simultaneously causing rapid burning of the fuel and subsequently fast rise in chamber pressure. The concept, while simple in light of this basic description, has proven to be very challenging. An in depth review of $\mathrm{HCCl}$ can be found in [65]. Some of the key highlights will be described here.

$\mathrm{HCCl}$ is of interest because it potentially combines the strengths of conventional spark and compression ignition counterparts. Use of $\mathrm{HCCl}$ offers the opportunity to maintain or increase efficiency comparable to diesel high compression engines while meeting or reducing harmful emissions levels similar to spark ignited gasoline engines. A HCCl engine has the potential to reduce cost and complexity, as there is less need for turbulence inducing piston and inlet shapes, no high pressure injection requirements, and no need for sophisticated after-treatment systems. $\mathrm{HCCl}$ combustion also offers a lower operating temperature platform compatible with a wide range of fuels [66]. 
Unfortunately, these benefits are accompanied by challenges that have not yet been met. Most notable of these is the lack of control available over the $\mathrm{HCCl}$ combustion event. Spark and compression ignition engines maintain spark and injection timing as highly effective control mechanisms - advancing or retarding the timing to affect engine performance. However, the $\mathrm{HCCl}$ event does not directly depend on either a spark or injection event. Instead, the onset of $\mathrm{HCCl}$ combustion is due to chemical kinetics and depends largely on pressure, temperature, stratification, and mixture composition. This challenge leads to another commonly cited problem: operation at $\mathrm{HCCl}$ conditions is constrained to a narrow load range. Practical implementation of $\mathrm{HCCl}$ will require the ability of the engine system to compensate for changes in load by affecting the $\mathrm{HCCl}$ event [66].

Progress towards control of $\mathrm{HCCl}$ combustion in conventional crankshaft-driven engines has been made through various investigations. Intake charge temperature regulation is investigated in [67, 68]. Simulated results show successful control of $\mathrm{HCCl}$ over a wide load range. Internal EGR is examined in [69] via numeric simulation to show successful control of combustion phasing and peak pressure. Of particular interest is the use of variable compression ratio in [68] as a viable control option for $\mathrm{HCCl}$. From experimentation with a multi-cylinder variable compression ratio engine, it is seen that higher compression can replace inlet air preheating as a control mechanism, but increased compression results in increased $\mathrm{CO}$ emissions. It was also seen that high load operation was limited by $\mathrm{NO}_{\mathrm{x}}$ emissions and noise, while low load operation was limited by misfire. This relates to the potential for the free piston engine (also with variable compression) to sympathetically operate with $\mathrm{HCCl}$ combustion where each cycle acts as feedback into the opposing cycle of the dual cylinder SALEG arrangement. Late ignition (relative to top dead center) leads to higher compression ratio while early ignition can result in negative work on the piston, thus reducing the overall system energy with the stroke and compression following. The free piston engine with variable compression also enables the potential to operate on a wide range of fuel types and under ultra-lean conditions. These potential applications and many others have been explored by researchers around the world, and the potential for the $\mathrm{HCCl}$ free piston engine is summarized well in the previously mentioned review by Mikalsen and Roskilly [9].

\subsubsection{Genetic Algorithm Optimization in Engineering}

The genetic algorithm (GA) is an optimization tool that is well-suited for large design spaces with multiple targets and constraints. The common genetic algorithm approach involves both small and large changes to model parameters while in search of a parameter set that leads to best performance. Small changes are often referred to as exploitation and large changes as exploration. These two tools search the available design space and can be combined with concepts such as elitism (wherein the 'best' solution is always kept among the possible solutions) to arrive at a better and better solution. The quality of any 
solution is quantified by the performance index which mathematically combines the desired operational outputs.

A GA is most suited for difficult optimization problems with large search spaces and could be applied to any field of study. Relevant to modeling and ICEs is a work by Polifke et al. which uses a GA to determine optimum rate coefficients for a simplified set of chemical kinetic reaction mechanisms used to describe the combustion of methane [70]. The GA made it possible to model lean-premixed, laminar methane combustion using two- or three- step mechanisms with good accuracy, especially at high pressures. Another application of GAs is the tuning of controller parameters. An example of this out of Tehran employs a GA to tune a fuzzy controller which minimizes fuel consumption and harmful emissions in a hybrid electric vehicle over simulated transient driving cycles [71]. A final example which speaks to the robust capabilities of a GA and the type of application that is pursued in the current research is found in [72]. Sullivan et al. employed a GA to optimize the mechanics and thermodynamics of a StephensonIII six-bar linkage (a possible replacement for the conventional slider-crank mechanism which drives the piston). Their final optimization routine required about two days of computational time and resulted in relatively small improvements over the slider-crank mechanism in terms of engine efficiency. This study relates to the current research closely because of its use of a GA to optimize a dynamic-thermodynamic system across many domains and parameters. In much the same way, a common GA approach is used to optimize the simulated SALEG system. 


\section{CH. 2 FUNDAMENTAL ANALYSIS}

As an initial step in understanding the nature of the system, a fundamental analysis was performed which resulted in various important insights. The analysis described here has been published in two parts $[4,5]$. This chapter reviews the fundamental analysis as described in these publications and lays the groundwork for the numeric simulation which follows.

\subsection{Basic System Description}

Modeling any complex system generally involves breaking down the system into smaller, more manageable pieces. A fundamental model represents the most basic combination of system processes. Such a model provides a foundation for understanding and a baseline for verification of other modeling efforts which may follow.

The most basic elements of this linear engine system are illustrated in Figure 6. A housing is divided into two cylinders. Two pistons are situated opposite each other within the housing and rigidly connected by a rod. Between the bottom of each piston head and the dividing wall of the housing are ideal compression springs. Also seen in Figure 6 are three geometric dimensions: $L_{C}$ represents the length of the cylinder from the system origin to the inside of the cylinder head; $L_{P}$ represents the length of one of the pistons from the center of the translator (pistons connected by rod) to the head of the piston; $b$ represents the bore (diameter) of each piston. Throughout the analysis, instantaneous position of the translator is measured with respect to the center of the symmetric engine housing, and subscripts $l$ and $r$ denote the left or right cylinder respectively.

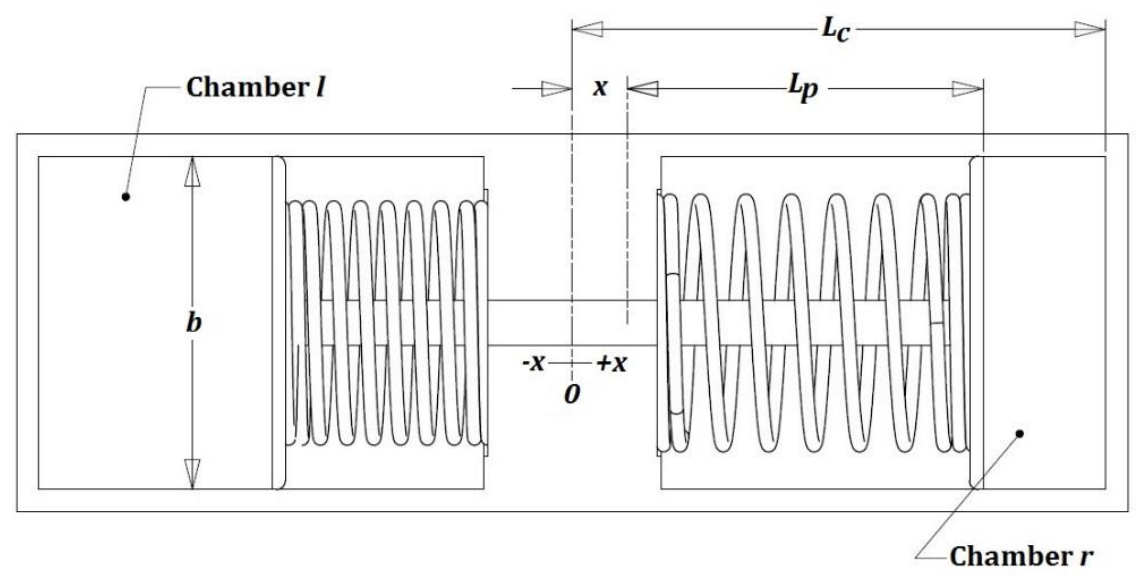

Figure 6: Illustration of simplified dual free piston linear engine system for use in fundamental analysis.

\subsection{Basic Relationships}

A number of initial assumptions must be made in order to build relationships which can describe the motion of the translator. These include: 
- No frictional losses

- Perfectly linear compression springs with no material restrictions

- No heat transfer

- Polytropic gas compression and expansion with equal and constant coefficients

- Ideal air with constant properties

- Perfectly sealed chambers

The geometric parameters can be related to find the instantaneous volume of either chamber through the following.

$$
\begin{gathered}
L=L_{C}-L_{P} \\
V_{l}=(L+x) \frac{\pi b^{2}}{4} \quad V_{r}=(L-x) \frac{\pi b^{2}}{4}
\end{gathered}
$$

The general polytropic relationship for compression and expansion of a gas is known as:

$$
P_{1} V_{1}^{\frac{C_{p}}{C_{v}}}=P_{2} V_{2}^{\frac{C_{p}}{C_{v}}}
$$

where the gas is characterized by the specific heat ratio (specific heat at constant pressure divided by the specific heat at constant volume). The subscripts 1 and 2 represent the first and second states of the gas respectively.

For this portion of the analysis, combustion and exhaust processes are not included so that if a pressure is known at any position, the pressure at any other position can be calculated. Because of device symmetry, the pressure in either chamber is equal when the translator is at its midpoint position $(x=0)$. Combining the volume relationships with the polytropic equality produces the following instantaneous pressure relationships for each chamber.

$$
P_{m p}\left(L \frac{\pi b^{2}}{4}\right)^{\gamma}=P_{l}\left[(L+x) \frac{\pi b^{2}}{4}\right]^{\gamma}=P_{r}\left[(L-x) \frac{\pi b^{2}}{4}\right]^{\gamma}
$$

Here, the subscript $m p$ indicates the midpoint pressure and the exponent $\gamma$ is substituted for the ratio of specific heats (assumed constant). Solving these relationships for the pressures in each chamber and scaling it according to the surface area of the piston head yields the pressure forces on the translator.

$$
F_{P, l}=\frac{\pi b^{2}}{4} P_{m p}\left(\frac{L}{L+x}\right)^{\gamma} \quad F_{P, r}=\frac{\pi b^{2}}{4} P_{m p}\left(\frac{L}{L-x}\right)^{\gamma}
$$

Because the springs are assumed to be perfectly linear and without mechanical limitations, the force in each spring can be related to the spring stiffness $(k)$, known free length of the spring $\left(L_{f l}\right)$, and instantaneous position of the translator $(x)$ relative to system geometry. The relationships for each spring are shown in Eq. 5. 


$$
\begin{aligned}
& F_{\text {spring, },}=k\left(L_{f l}-\left|x-L_{P}\right|\right)=k\left(L_{f l}+\left(x-L_{P}\right)\right) \\
& F_{\text {spring, },}=k\left(L_{f l}-\left|x+L_{P}\right|\right)=k\left(L_{f l}-\left(x+L_{P}\right)\right)
\end{aligned}
$$

The absolute value quantities represent the deflection of the springs dependent on the translator position and can be simplified based on the physical limitation that the left piston will never reach a position to the right $(x \ngtr 0)$ of the origin and vice versa for the right piston. A free body diagram helps to demonstrate how the forces are applied to the translator. This is shown in Figure 7.

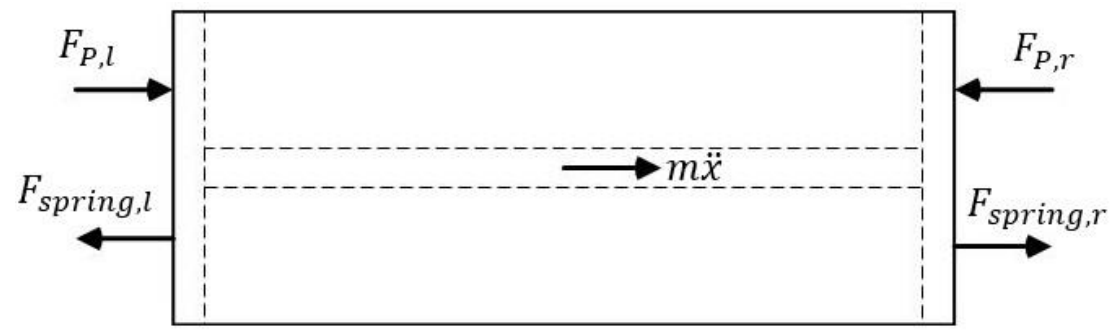

Figure 7: Free body diagram of simple translator for fundamental analysis without friction or work terms.

Summing the forces according to Newton's second law and simplifying gives a second order differential equation in time for the position of the translator. This is shown in Eq. 6.

$$
\begin{gathered}
\frac{\pi b^{2}}{4} P_{m p}\left[\left(\frac{L}{L+x}\right)^{\gamma}-\left(\frac{L}{L-x}\right)^{\gamma}\right]-2 k x=m \ddot{x} \\
x=x(t)
\end{gathered}
$$

From the free body diagram presented in Figure 7, Eq. 6 represents the time dependent motion of the translator as it travels from some position left of the origin to the equal but opposite position right of the origin. The solution, however, is intractable because of its nonlinearity and requires manipulation to reach a non-trivial, closed form solution. The first step is to define nondimensional terms within the equation which can parametrically represent the system. The first of these has already been supplied.

- The ratio of specific heats:

$$
\gamma=\frac{C_{P}}{C_{V}}
$$

- Dimensionless translator position bounded by -1 to the left and 1 to the right:

$$
x^{*}=\frac{x}{L}
$$

- Dimensionless pressure, in this case, the midpoint pressure:

$$
P_{m p}^{*}=\frac{\pi b^{2} P_{m p}}{4 L k}
$$

- Characteristic time: 


$$
t^{*}=\frac{t}{\sqrt{m / k}}
$$

Introducing these terms into Eq. 6 yields the following (Eq. 11).

$$
P_{m p}^{*}\left[\frac{1}{\left(1+x^{*}\right)^{\gamma}}-\frac{1}{\left(1-x^{*}\right)^{\gamma}}\right]-2 x^{*}=\frac{d^{2} x^{*}}{d t^{* 2}}=a^{*}
$$

In Eq. 11, the dimensionless acceleration is introduced as the second time derivative of the dimensionless position. A substitution (shown in Eq. 12) now introduces the dimensionless velocity.

$$
\begin{gathered}
v^{*}=\frac{d x^{*}}{d t^{*}} \quad \Longrightarrow \quad \frac{d^{2} x^{*}}{d t^{*}}=v^{*} \frac{d v^{*}}{d x^{*}} \\
P_{m p}^{*}\left[\frac{1}{\left(1+x^{*}\right)^{\gamma}}-\frac{1}{\left(1-x^{*}\right)^{\gamma}}\right]-2 x^{*}=v^{*} \frac{d v^{*}}{d x^{*}}
\end{gathered}
$$

The new form shown in Eq. 13 can be solved to produce a closed form solution of the dimensionless velocity with respect to the dimensionless position of the translator. Performing the integration yields Eq. 14.

$$
\begin{gathered}
\frac{1}{2} v^{* 2}=\frac{-P_{m p}^{*}}{\gamma-1}\left[\left(1+x^{*}\right)^{1-\gamma}+\left(1-x^{*}\right)^{1-\gamma}\right]-x^{* 2}-C^{*} \\
C^{*}=\frac{-P_{m p}^{*}}{\gamma-1}\left[\left(1+x_{l}^{*}\right)^{1-\gamma}+\left(1-x_{l}^{*}\right)^{1-\gamma}\right]-x_{l}^{* 2}
\end{gathered}
$$

Eq. 14 presents the case where no energy is added to or removed from the system, so the final position of the translator will be an equal distance from the origin but opposite of the starting position. The constant of integration comes from the initial condition which states that at some initial position to the left of the origin $\left(x=x_{l}^{*}\right)$, the velocity is equal to zero $\left(v^{*}=0\right)$. The solution in Eq. 14 is also limited by a similar boundary condition which recognizes that the translator velocity will return to zero at some point to the right of the origin $\left(v^{*}\left(x_{r}^{*}\right)=0\right)$. The first of the author's publications [4] explores this system in greater detail (spring versus pressure limiting cases), but in any case the velocity profile is symmetric with respect to the position origin such that $x_{l}^{*}=-x_{r}^{*}$ and about the velocity axis such that $v^{*}(0)=v_{\text {max }}^{*}$.

\subsection{Basic Heat and Work}

Building upon the most basic system, it is now possible to move towards a more interesting device which reacts to the addition and subtraction of energy during its operation. To maintain simplicity, the following assumptions are made.

- Heat is added and rejected at top and bottom dead center, respectively

- Heat addition and rejection occur instantaneously

- Isentropic expansion and compression of cylinder gases is maintained (no heat transfer)

- Work output is represented as a constant energy removal over the length of the stroke 
With these assumptions, an initial position to the left of the origin $\left(-1<x_{l}^{*}<0\right)$, and a known midpoint pressure, the pressure and volume in each chamber can be calculated. Focusing attention on the left chamber at this initial state, pressure and volume are given by the following.

$$
P_{l, 1}=P_{m p}\left(\frac{L}{L+x_{l}}\right)^{\gamma} \quad V_{l, i}=\left(L+x_{l}\right) \frac{\pi b^{2}}{4}
$$

For any given stroke, when the translator reaches its left-most position, the left piston is at its top dead center (TDC) location. Under the previously outlined assumptions, constant volume heat addition occurs at each top dead center event. Thermodynamic first law energy analysis for a closed system allows the cylinder pressure to be related to the heat addition through the following.

$$
Q_{\text {in }}=\Delta U=m_{g a s} C_{v}\left(T_{2}-T_{1}\right)
$$

Eq. 16

In the previous relationship, $T$ represents the temperature of the gas with subscripts 1 and 2 again representing the first and second state respectively. Also, the heat added $\left(Q_{\text {in }}\right)$, change in internal energy $(\Delta U)$, and mass of the gas $\left(m_{\text {gas }}\right)$ are represented. Because the properties and mass of the ideal gas within each chamber remain constant, the following substitutions can be made.

$$
Q_{\text {in }}=m_{\text {gas }} C_{v}\left(\frac{P_{l, 2} V_{l, i}}{m_{\text {gas }} R}-\frac{P_{l, 1} V_{l, i}}{m_{\text {gas }} R}\right)=\frac{V_{l, i} C_{v}}{R}\left(P_{l, 2}-P_{l, 1}\right)
$$

The ideal gas constant $(R)$ can be related to the specific heat according to the following.

$$
R=C_{P}-C_{V}
$$

This enables the following substitution.

$$
Q_{i n}=\frac{V_{l, i} C_{v}}{C_{p}-C_{v}}\left(P_{l, 2}-P_{l, 1}\right)=\frac{V_{l, i}}{\gamma-1}\left(P_{l, 2}-P_{l, 1}\right)
$$

Then, the initial pressure and volume relationships are applied.

$$
Q_{\text {in }}=\left(\frac{\pi b^{2}}{4}\right)\left(\frac{L+x_{l}}{\gamma-1}\right)\left[P_{l, 2}-P_{m p}\left(\frac{L}{L+x_{l}}\right)^{\gamma}\right]
$$

Rearranging this to solve for the pressure at state two, that is to say the pressure after the heat has been added to the cylinder, the following relationship is formed.

$$
P_{l, 2}=Q_{i n} \frac{4(\gamma-1)}{\pi b^{2}\left(L+x_{l}\right)}+P_{m p}\left(\frac{L}{L+x_{l}}\right)^{\gamma}
$$

So, with the cylinder pressure known at top dead center, the polytropic relationship can be applied to calculate the pressure and related force on the translator at any position.

$$
P_{l, 2} V_{l, i}^{\gamma}=P_{l} V_{l}^{\gamma}
$$




$$
\begin{aligned}
& P_{l}=\left[Q_{\text {in }} \frac{4(\gamma-1)}{\pi b^{2}\left(L+x_{l}\right)}+P_{m p}\left(\frac{L}{L+x_{l}}\right)^{\gamma}\right]\left(\frac{L+x_{l}}{L+x}\right)^{\gamma} \\
& F_{P, l}=\frac{Q_{\text {in }}(\gamma-1)\left(L+x_{l}\right)^{\gamma-1}}{(L+x)^{\gamma}}+\frac{\pi b^{2}}{4} P_{m p}\left(\frac{L}{L+x}\right)^{\gamma}
\end{aligned}
$$

A work term (meant to represent a combination of friction and useful work out of the system) is added to the free body diagram shown in Figure 7 to arrive at Figure 8.

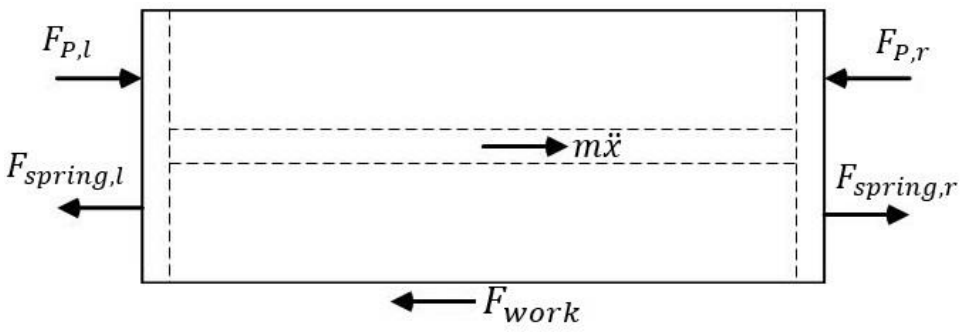

Figure 8: Free body diagram of translator with resistive work force applied.

Summing the forces shown in Figure 8 and simplifying, Eq. 25 is formed to represent the motion of the translator from left to right with heat added to the left cylinder according to Eq. 24 and work removed during the stroke.

$$
\frac{Q_{i n}(\gamma-1)\left(L+x_{l}\right)^{\gamma-1}}{(L+x)^{\gamma}}+\frac{\pi b^{2}}{4} P_{m p}\left[\left(\frac{L}{L+x}\right)^{\gamma}-\left(\frac{L}{L-x}\right)^{\gamma}\right]-2 k x-F_{W}=m \frac{d^{2} x}{d t^{2}}
$$

This is similar in form to Eq. 6 with the addition of the heat and work terms previously described. Again forming nondimensional terms, Eq. 25 can be reduced to Eq. 26

$$
Q_{i n}^{*}\left[\frac{(\gamma-1)\left(1+x_{l}^{*}\right)^{\gamma-1}}{\left(1+x^{*}\right)^{\gamma}}\right]+P_{m p}^{*}\left[\frac{1}{\left(1+x^{*}\right)^{\gamma}}-\frac{1}{\left(1-x^{*}\right)^{\gamma}}\right]-2 x^{*}-F_{W}^{*}=\frac{d^{2} x^{*}}{d t^{* 2}}=v^{*} \frac{d v^{*}}{d x^{*}}=a^{*}
$$

where the nondimensional heat addition and work are given by the following two terms respectively.

$$
Q_{\text {in }}^{*}=\frac{Q_{\text {in }}}{k L^{2}} \quad F_{W}^{*}=\frac{F_{W}}{k L}
$$

Again applying the velocity transformation given in Eq. 12 and integrating with the zero velocity initial condition $\left(v^{*}\left(x_{l}^{*}\right)=0\right)$, the closed form solution of Eq. 26 yields the relationship between position and velocity shown in Eq. 28.

$$
\begin{gathered}
\frac{1}{2} v^{* 2}=-Q_{i n}^{*}\left[\left(\frac{1+x_{l}^{*}}{1+x^{*}}\right)^{\gamma-1}\right]-\frac{P_{m p}^{*}}{\gamma-1}\left[\left(1+x^{*}\right)^{1-\gamma}+\left(1-x^{*}\right)^{1-\gamma}\right]-x^{* 2}-F_{W}^{*} x^{*}-C^{*} \\
C^{*}=-Q_{i n}^{*}-\frac{P_{m p}^{*}}{\gamma-1}\left[\left(1+x_{l}^{*}\right)^{1-\gamma}+\left(1-x_{l}^{*}\right)^{1-\gamma}\right]-x_{l}^{* 2}-F_{W}^{*} x_{l}^{*}
\end{gathered}
$$

This relationship represents the travel of the translator effected by instantaneous heat addition in the left cylinder at TDC and constant work taken out over the length of the stroke. To accomplish a full 
cycle of engine operation, heat rejection is accomplished in the left cylinder by returning the pressure to the midpoint pressure polytropic curve which is analogous to a motoring curve. This instantaneous heat rejection acts as a highly idealized exhaust/scavenging process and is independent of position except that the motoring curve varies with position. The process effectively dissipates any energy within the cylinder in the form of internal gas energy and occurs when the left piston is at its bottom dead center (BDC) position. Left cylinder BDC position coincides with right cylinder TDC position. At this position, heat is added to the right cylinder in exactly the same fashion and amount as described above for instantaneous heat addition to the left cylinder. The right dead center position is identified as $x_{r}^{*}$, and, through nearly the same analysis as was applied to find Eq. 28, the following closed form solutions are found for the dimensionless position, velocity (Eq. 30), and acceleration (Eq. 29) of the translator as it travels from right to left.

$$
\begin{gathered}
a^{*}=v^{*} \frac{d v^{*}}{d x^{*}}=Q_{i n}^{*}\left[\frac{(\gamma-1)\left(1-x_{r}^{*}\right)^{\gamma-1}}{\left(1-x^{*}\right)^{\gamma}}\right]+P_{m p}^{*}\left[\frac{1}{\left(1-x^{*}\right)^{\gamma}}-\frac{1}{\left(1+x^{*}\right)^{\gamma}}\right]+2 x^{*}-F_{W}^{*} \\
\frac{1}{2} v^{* 2}=Q_{i n}^{*}\left[\left(\frac{1-x_{r}^{*}}{1-x^{*}}\right)^{\gamma-1}\right]+\frac{P_{m p}^{*}}{\gamma-1}\left[\left(1+x^{*}\right)^{1-\gamma}+\left(1-x^{*}\right)^{1-\gamma}\right]+x^{* 2}-F_{W}^{*} x^{*}-C^{*} \\
C^{*}=Q_{i n}^{*}+\frac{P_{m p}^{*}}{\gamma-1}\left[\left(1+x_{r}^{*}\right)^{1-\gamma}+\left(1-x_{r}^{*}\right)^{1-\gamma}\right]+x_{r}^{* 2}-F_{W}^{*} x_{r}^{*}
\end{gathered}
$$

The translator returns from the right at position $x_{r}^{*}$ with a negative velocity to a position left of the origin (according to Eq. 30). When the left dead center position is reached again, the instantaneous heat addition and heat rejection processes are applied to the left and right cylinders respectively. The cycle can continue in this fashion to achieve one of three final states.

The three cases are explored in detail in [4] and are more briefly characterized here. The first case demonstrates how adding more energy to the system than that which is being removed results in a rise of compression ratio (shown in Figure 9). Over each cycle, the stroke increases and the energy within each cylinder rises. In a practical setting, uncontrolled runaway of compression ratio could quickly result in mechanical failure of engine components. 

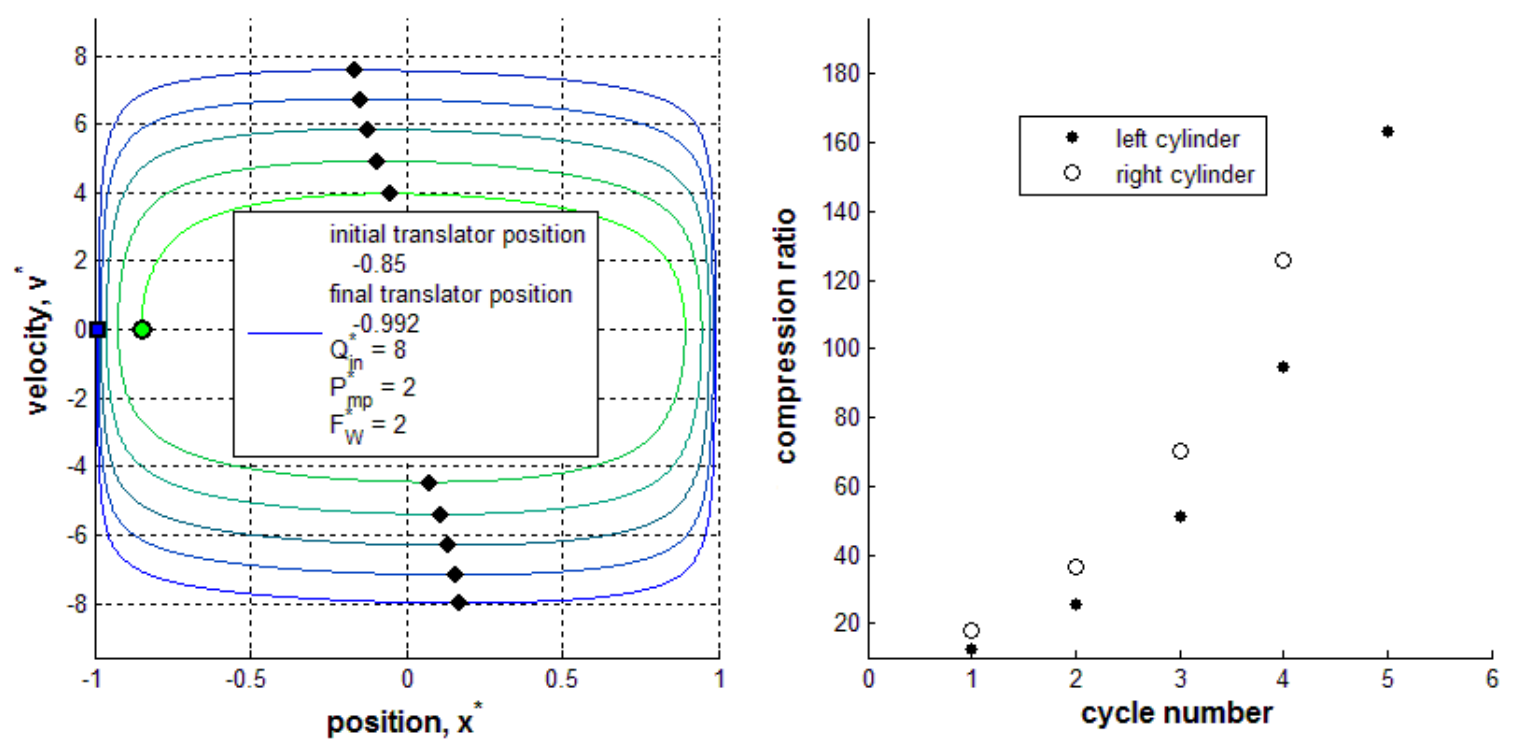

Figure 9: Basic free piston system including dimensionless heat and work [4]. Compression ratio rises as a result of energy imbalance.

In the next case, the opposite result is demonstrated. That is, during each stroke, more energy is removed through work and heat rejection than is added in heat addition. This results in a dampening of the oscillation and decreasing compression ratio as shown in Figure 10.
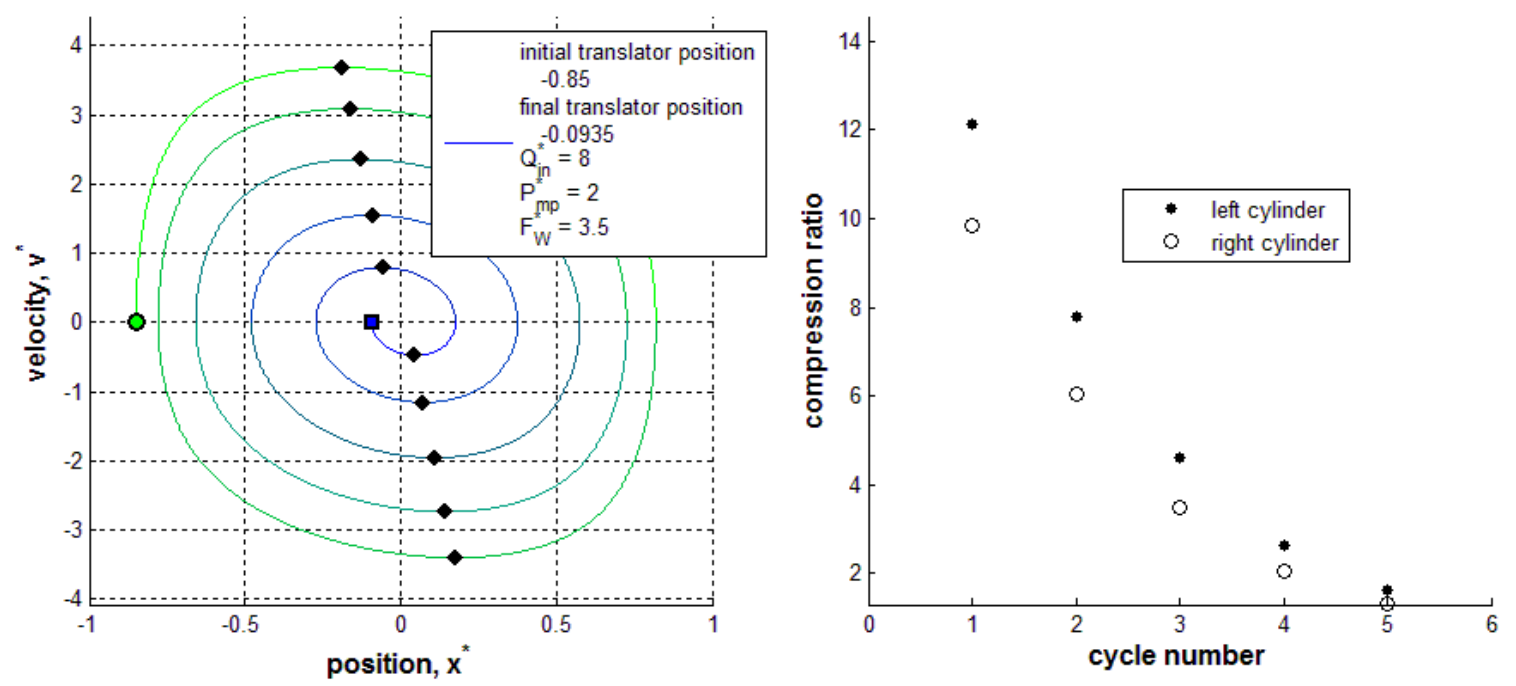

Figure 10: Basic free piston system including dimensionless heat and work [4]. Compression ratio falls as a result of energy imbalance.

The third and final case described here is that of cyclic stability or equilibrium. The first example for this is shown in Figure 11. For the given heat, work, and starting position input parameters, the compression ratio rises with each stroke towards stable operation. 

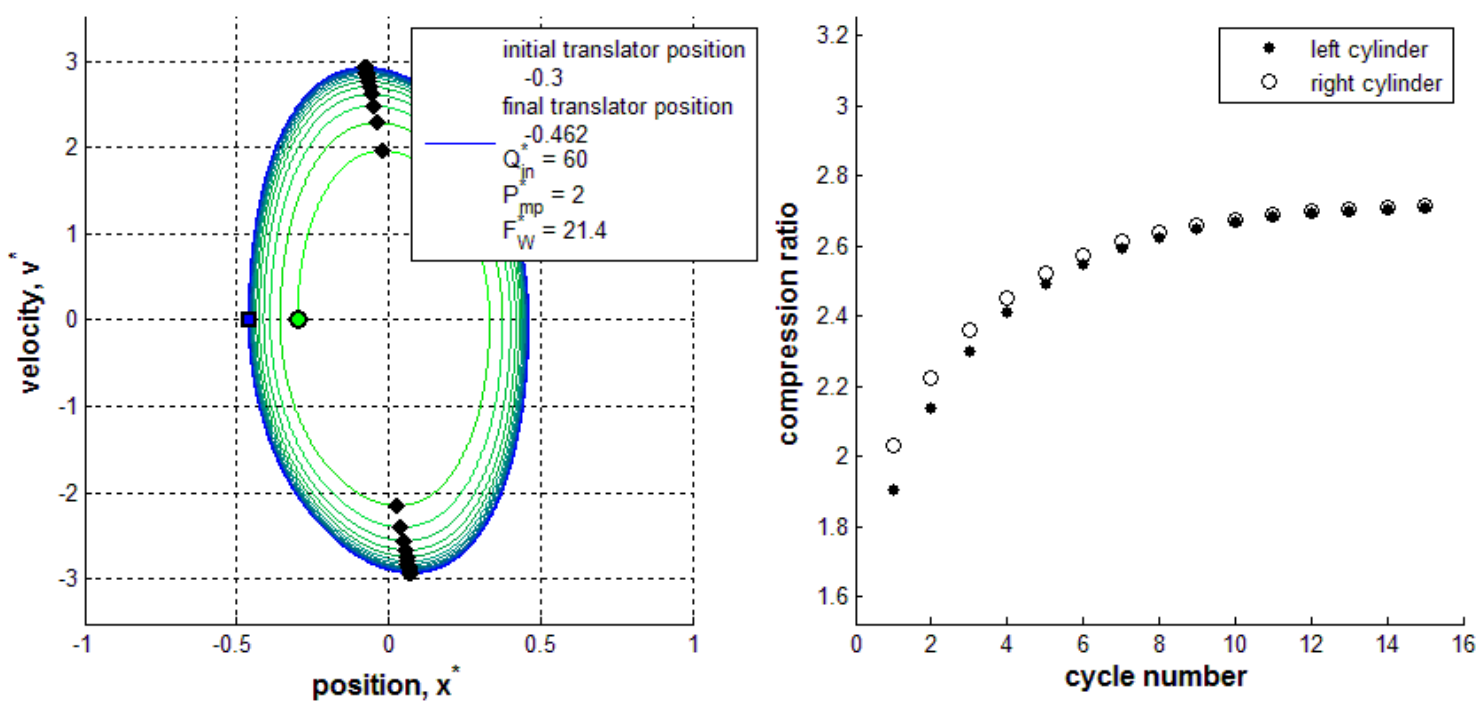

Figure 11: Basic free piston system including dimensionless heat and work [4]. Energy is balanced sufficiently for compression to rise to stability.

In the next example, the same input parameters are applied except for the starting position of the translator. Figure 12 shows the operation of this system. Noting the final translator position shown in Figure 11 and comparing it with that in Figure 12 makes it apparent that the same stable compression ratio is reached in both cases.
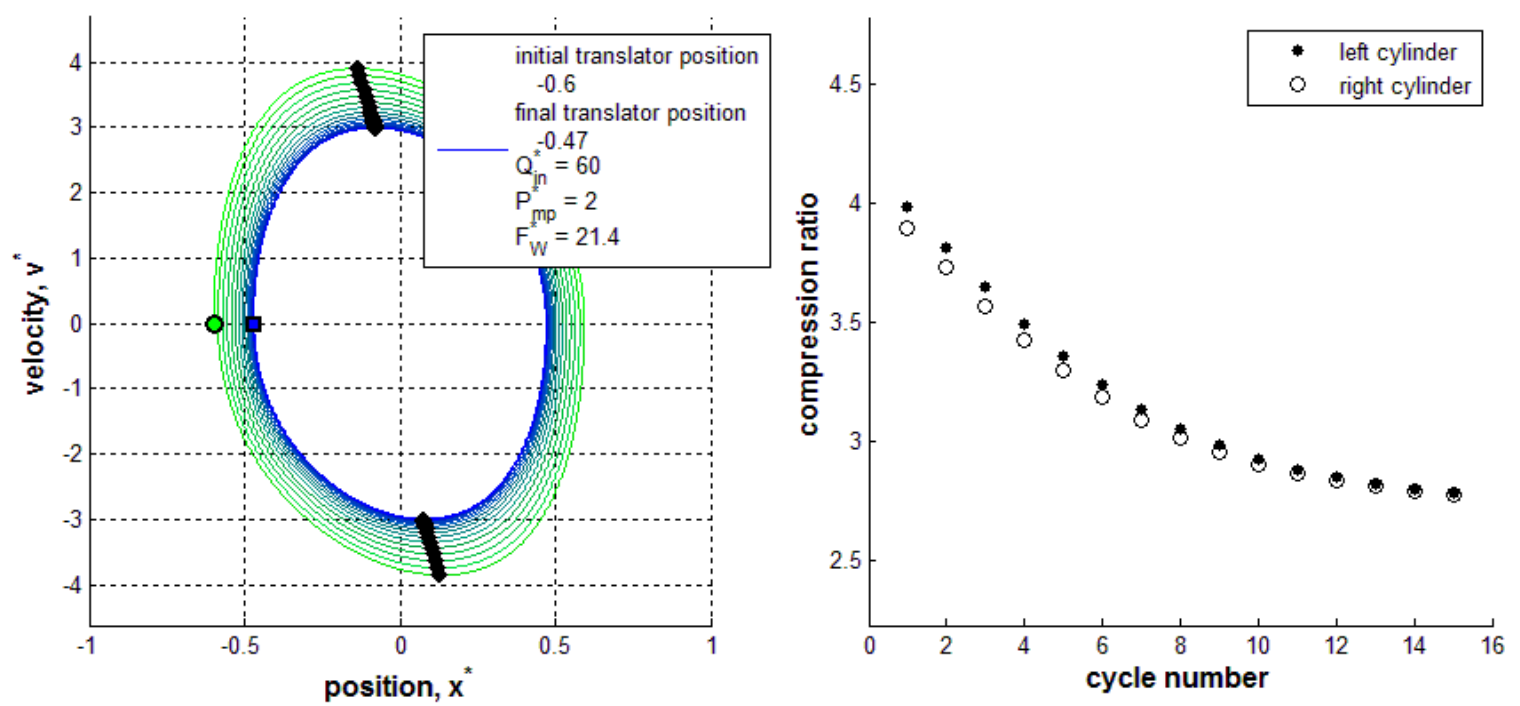

Figure 12: Basic free piston system including dimensionless heat and work [4]. Energy exchange is sufficiently balanced for compression to fall to stability.

Further exploration of the equilibrium case allows for the development of a relationship between the heat added and work removed from the engine system. Based on Otto cycle efficiency for an engine (with the cold air assumption in tow), the ideal amount of energy removed from the system is a function of the heat addition and compression ratio. With this relationship, equilibrium operation can be attained for any known heat input at any given compression. This relationship is shown in Eq. 31. 


$$
F_{W}^{*}=\frac{Q_{i n}^{*}}{x_{r}^{*}-x_{l}^{*}}\left[1-\left(\frac{1+x_{l}^{*}}{1+x_{r}^{*}}\right)^{\gamma-1}\right]
$$

In this form, the compression ratio is present in terms of the starting and ending position of the stroke. Constraining the work energy by this equation guarantees system stability. An example of this application is seen in Figure 13.
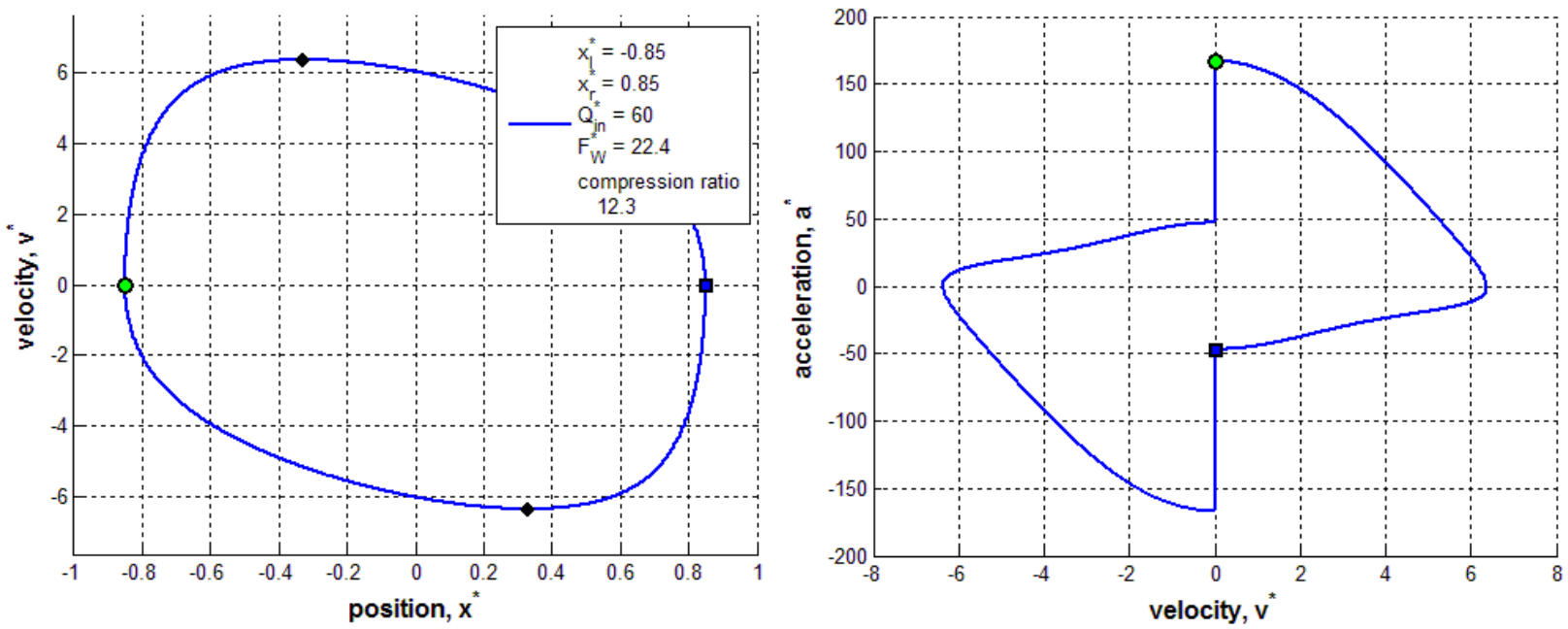

Figure 13: Basic free piston system including dimensionless heat and work [4]. Energy is constrained to operate at equilibrium by the Otto cycle efficiency.

In the equilibrium illustrated by Figure 13, the plot makes it evident that the left dead center and right dead center positions are equal but opposite in their distance from the origin. This $\left(x_{r}^{*}=-x_{l}^{*}\right)$ can be applied to simplify Eq. 31 further.

$$
F_{W}^{*}=\frac{Q_{i n}^{*}}{-2 x_{l}^{*}}\left[1-\left(\frac{1+x_{l}^{*}}{1-x_{l}^{*}}\right)^{\gamma-1}\right]
$$

The application of Eq. 32 is explored in some detail in [4], and it is shown that for any compression ratio (directly related to a known starting position at left dead center position), natural equilibrium involves a very narrow band of tolerance for the proportional amounts of heat and work exchanged within and without the system.

These fundamental concepts of simple heat work and their effects on natural equilibrium are important for building a basic understanding of the dual piston linear engine system. And, arriving at a closed form solution is appealing as it excludes any uncertainty which could be introduced in the numerical solution of a system of nonlinear equations. Next, the model is extended, still within the fundamental (nondimensional) space, to gain insight into the effects of a work profile which includes position dependence, combustion prior to top dead center, and non-adiabatic cylinder 
compression/expansion. These sophistications are more fully described in [5] and will be succinctly detailed in the next few sections.

\subsection{Spatially Dependent Work Profile}

The first step taken to extend the basic model is to develop a dimensionless work force profile which depends on the dynamics of the translator. In the basic system previously examined, the work force was assumed to be constant throughout the entire stroke. Here, a more realistic profile is employed to study the effects of spatially dependent forces on the velocity and acceleration profiles.

In [73], Aichlmayr and Van Blarigan validate a model for the magnetic flux of the alternator as a function of the translator position. In another work [12], Van Blarigan and researchers consider the force exerted by the alternator to be a function of translator velocity. While this is not a contradiction, it does demonstrate that the varied approaches which might be taken to represent a dependent work profile. In this fundamental effort, the velocity of the translator is not known a priori, so to maintain a closed form solution, the work force is constructed as a function of position only. In [8], Aichlmayr provides typical work profiles which might be seen in a free piston alternator device. Based on this, a cosine function is selected of the following form.

$$
A \cos \left(\frac{\pi}{2} x^{*}\right)
$$

Eq. 33

To maintain equilibrium operation of the system, the Otto efficiency is applied as seen in Eq. 31 . Equating the stroke integrals of the two work curves (constant and cosine) ensures that the work output of the system is constrained by the Otto efficiency and allows for the solution of the coefficient $A$ [5]. This relationship is given in Eq. 34 .

$$
A=\frac{\pi F_{W}^{*}\left(x_{r}^{*}-x_{l}^{*}\right)}{2\left[\sin \left(\frac{\pi}{2} x_{r}^{*}\right)-\sin \left(\frac{\pi}{2} x_{l}^{*}\right)\right]}
$$

In the relationship found for the work force amplitude $(A)$, the dimensionless work force constant $\left(F_{W}^{*}\right)$ is calculated using Eq. 31 allowing for easy employment of Eq. 33 to find the work force applied to the translator as a function of the dimensionless translator position. An example of this is seen in Figure 14 and compared to the originally constant work profile. 


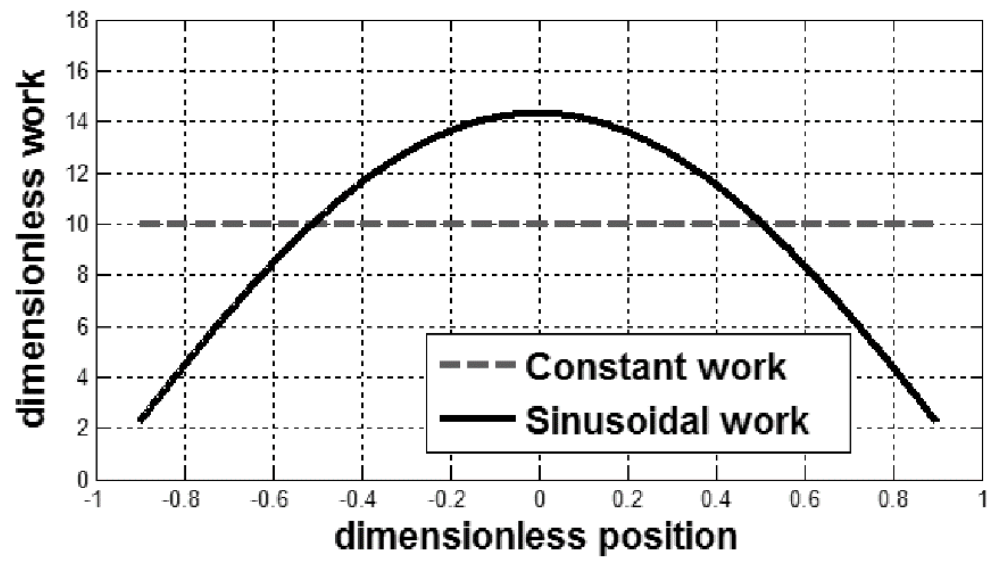

Figure 14: Constant work force profile compared to the cosine profile [5]. The integral of each profile over the position is constrained to be equivalent.

Substitution of Eq. 33 in place of the work force term $\left(F_{W}^{*}\right)$ of Eq. 26 and Eq. 29 and then integrating to find the translator velocity as a function of position allows for engine cycle simulation. The effects of the sinusoidal work profile are demonstrated in Figure 15.
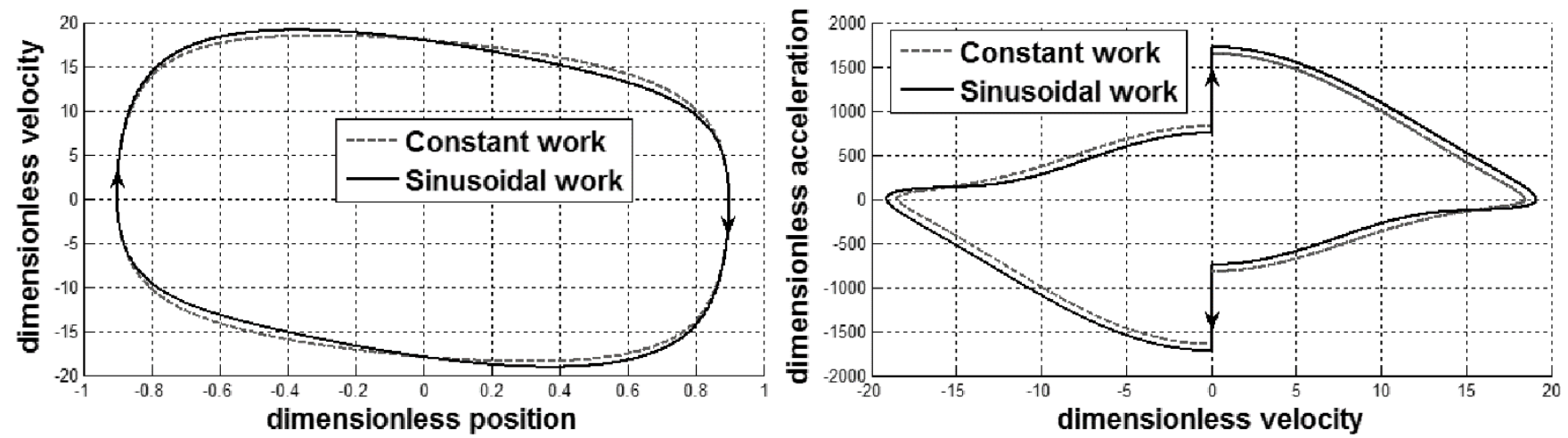

Figure 15: Comparison of the effects of constant and cosine work profiles on translator dynamics [5].

As seen in Figure 15, peak velocity and acceleration of the translator slightly increases with the use of the cosine work profile. This is due to the reduced amount of resistive work force applied to the translator near its dead center positions $\left(v^{*}=0\right)$. Subsequently, the translator accelerates more rapidly towards a greater velocity. Then, around the middle of the stroke $\left(x^{*}=0\right)$, the work force is greater in the case of the cosine profile causing a slightly more rapid decline in velocity. The effects seen are relatively small given any set of operating conditions because given those operating conditions, the work force profile is dictated to be of the same order of magnitude. Further, in any case, the Otto efficiency constraint guarantees the equilibrium operation of the engine system and equal amount of work extracted regardless of the work force profile. Next is discussed the system in which combustion is allowed to occur before piston top dead center. 


\subsection{Combustion Timing Prior to TDC}

Up to this point in the fundamental analysis, two major simplifications have allowed the closed form model with heat addition from combustion. First, combustion has been set to occur at TDC regardless of any conditions within the respective cylinder. In reality, combustion timing is very much dependent upon cylinder pressure and temperature as well as fuel and air mixture conditions. Second, the combustion event has been made to occur instantaneously - manifested as a constant volume change in pressure which basically represents the extremely rapid heat release during a $\mathrm{HCCl}$ combustion event [74].

Adding sophistication to the model while maintaining the closed form analytical solution, the combustion event is kept as a constant volume process, but the combustion timing can be augmented. In a true $\mathrm{HCCl}$ engine, combustion is initiated when temperature, pressure, and fuel composition conditions result in the spontaneous ignition at many points within the cylinder volume. In practical applications, injection timing can be used to retard ignition until after TDC [75]. However, if fuel is injected early during the compression stroke, achieving better homogeneity of the fuel-air mixture, ignition timing is constrained to before or at TDC as the highest pressure and temperature combination will be reached at TDC.

To allow for combustion at a location other than TDC in the fundamental model, the first assumption is that ignition will occur before or up to TDC. To maintain the simplicity of the model, pressure is assumed a suitable surrogate for temperature while mixture conditions are assumed homogeneous. So, during any given stroke, ignition occurs if a prescribed pressure threshold is reached. This threshold is defined relative to the pressure reached in the combustion cylinder at the initial position. The relationships that define subsequent strokes are discretized [5], allowing for closed form solution between discrete steps in position as well as access to cylinder pressure information for combustion initiation. During each stroke, a conditional statement examines the in-cylinder pressure, and if the pressure threshold is reached, heat is added to represent the constant volume combustion process.

As a first example, Figure 15 could represent the simulation of this system in which $100 \%$ of the initial pressure was required to achieve combustion. Because heat and work were related through the Otto cycle efficiency, the pressure threshold was reached during each stroke at exactly top dead center and thus no effects are seen. In another example (simulation results seen below in Figure 16), the combustion pressure threshold was set to $20 \%$ of the initial cylinder pressure to cause combustion significantly prior to TDC. The combustion event is manifested by a sharp change in the velocity profile caused by the constant volume heat release and subsequent instantaneous change in translator acceleration. In the position velocity plot from Figure 16, the abrupt change is most evident during the 
first few strokes and can be seen in the top right and bottom left of the curve - circled areas in the positionvelocity plot, just before top dead center positions.
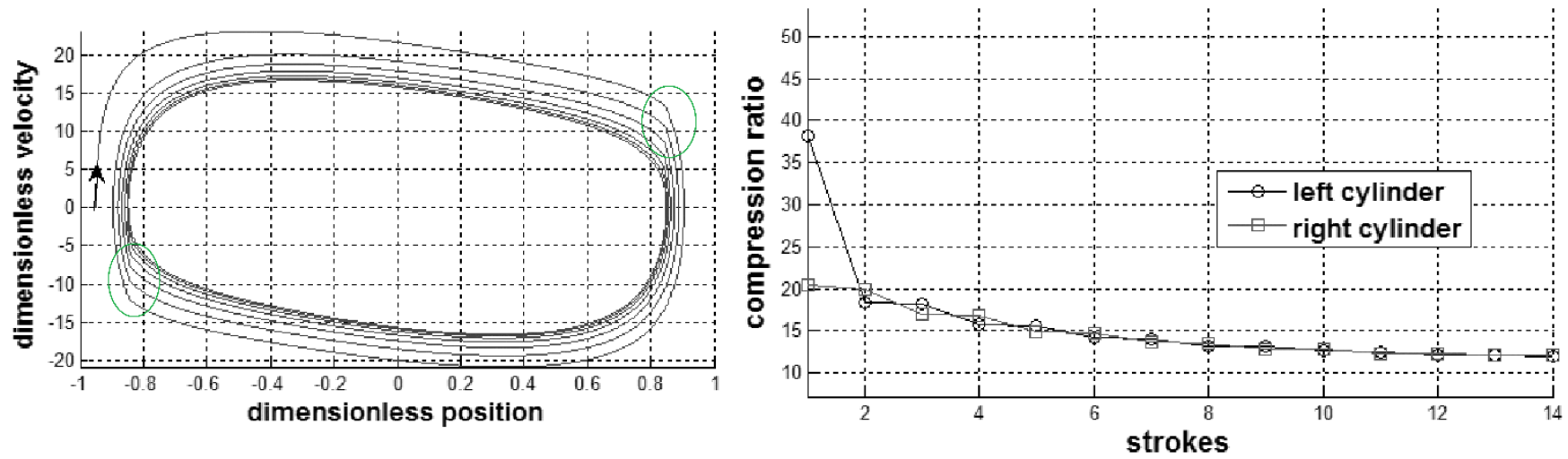

Figure 16: Fundamental simulations where the combustion pressure at $20 \%$ causes combustion to occur prior to top dead center [5].

Also observable in the Figure 16 plots is the decrease of the compression of the engine towards equilibrium operation with TDC combustion. This trend holds for any set of operating parameters with combustion occurring prior to top dead center.

\subsection{Non-Adiabatic Expansion and Compression}

In the basic system, one of the most significant simplifications was the neglecting of heat transfer during cylinder compression and expansion processes. While the theoretical Otto cycle includes isentropic pressure changes (polytropic, adiabatic) for each cylinder, a realistic engine loses heat to the cylinder walls and piston head during both compression and expansion. To achieve this sophistication in the fundamental model, the polytropic exponent $(n)$ is altered such that it no longer equals the specific heat ratio and varies between the compression and expansion processes. This is a simple approach with respect to the large body of research dedicated to studying engine heat transfer, but the next simulations demonstrate its effectiveness. First, Figure 17 illustrates the effect of the polytropic pressure curves during a single cycle simulation with combustion prior to TDC. The curves are compared to the adiabatic pressure curves which have been applied up to this point. 

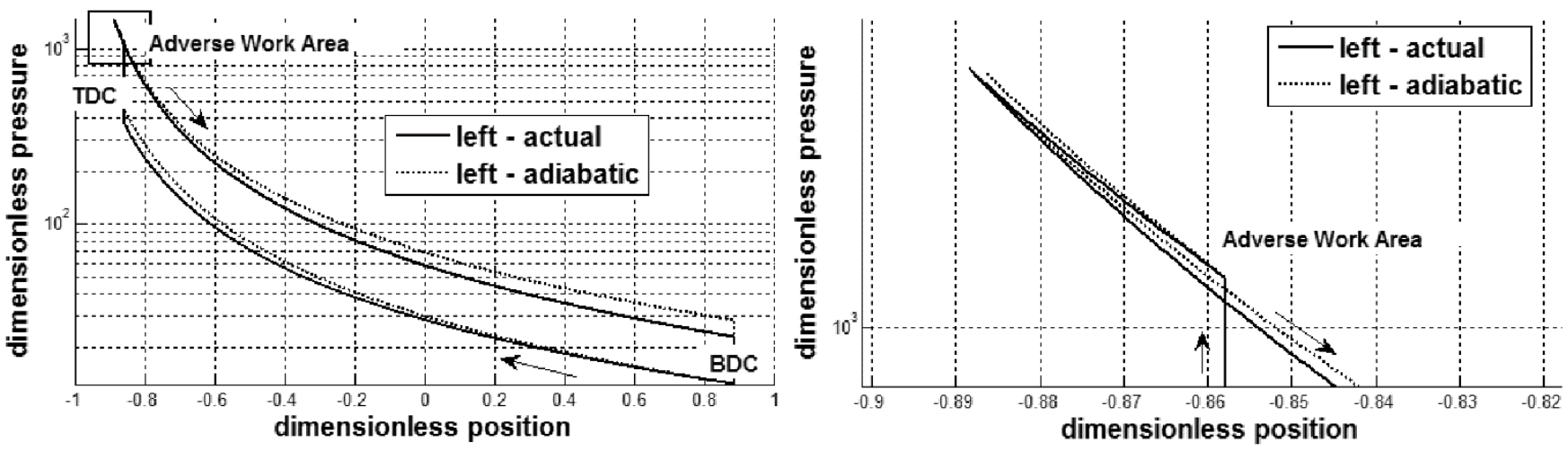

Figure 17: Pressure curves to demonstrate heat transfer and adverse work [5]. The adiabatic profile is compared to the polytropic profiles. Advanced combustion timing and heat transfer combine to allow adverse work.

Energy lost due to heat transfer is evident in the plots through the decreased amount of cylinder pressure in the actual (polytropic) case than in the adiabatic (previously used) case. With the inclusion of pre-TDC combustion, this also allows for adverse work, seen in the zoomed plot as an inversion of the pressure curve. This adverse work is energy further lost in the system because it is consumed in turning the translator around for the next stroke. Previously, adverse work was not present because the cylinder pressure followed the same curve during both expansion and compression processes.

\subsection{Combined Effects}

In combining the effects of these three sophistications, a large number of system responses can be achieved. These are more fully explored in [5] leading to the need to balance heat and work exchanges to maintain equilibrium oscillation. To achieve equilibrium in the simulation, a second law thermodynamic efficiency is applied to the work output to balance the heat transfer and adverse work losses. The position, velocity, and acceleration profiles of a high compression simulation are shown in Figure 18.
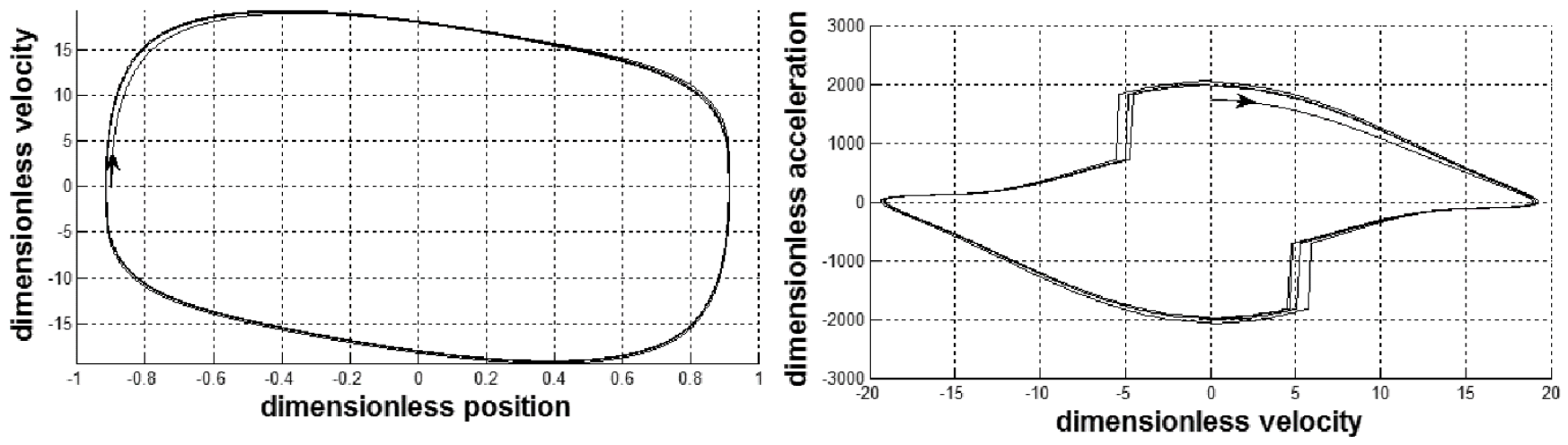

Figure 18: Dynamic profiles for multi-cycle simulation [5]. Fundamental analysis concepts are combined for a high compression equilibrium simulation.

Due to the high compression ratio, the translator residence time around TDC is very short. This is indicated by the steep sides of the position-velocity curve. The combustion event prior to TDC is evident 
in the acceleration profile as the sharp increase before velocity equals zero. Figure 19 gives two additional plots from the same simulation to help further describe the nature of the system.

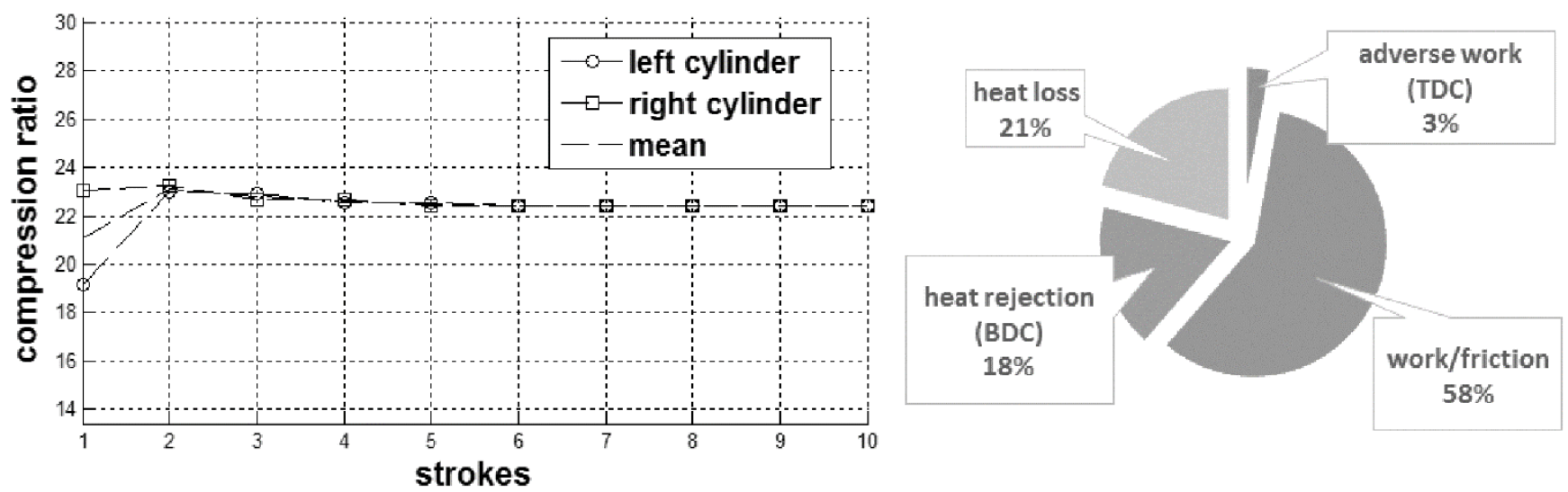

Figure 19: Transient settling of compression ratio and energy balance for multi-cycle simulation [5] - continued from Figure 18.

The initial transience of the system are seen in the variation of the compression ratio as the system settles into its natural dynamic equilibrium operation. In the right hand plot of Figure 19, the results of an energy analysis of the final equilibrium cycle are shown to quantify the simulation. In the chart, energy lost is accounted for in heat transfer losses, heat rejection at BDC, and adverse work around TDC. The work and friction were not handled independently in the model, but if a portion of this were taken to be friction - possibly on the order of $<10 \%$ for the SALEG system [76], the work output and thus overall efficiency would be just under $50 \%$. This is consistent with the high compression ratio application.

The trends and fundamental relationships developed throughout Ch. 2 and the two associated publications [4, 5] offer a foundational platform and verification tool for the numeric and Simulink models. The concept of adverse work around top dead center is demonstrated to be the result of early combustion and heat transfer - i.e. advancing the combustion timing or allowing for high heat transfer both increase the adverse work. Early combustion is shown to reduce stroke length. And, the shape of the work force profile is shown have small effects on the dynamics of the translator. Finally, the relationships - such as the Otto cycle stability relationship between heat and work - might be applied to parametrically study the design and optimization of the SALEG. 


\section{CH. 3 NUMERIC SIMULATION}

Because this research is highly dependent upon simulations of the SALEG, significant effort has been made to develop a sophisticated and robust virtual model. The primary outcome of the modeling is increased understanding of the operation of the device over many cycles at steady state and during transient events. To achieve this, each sub-model is as sophisticated as computationally allowable. For example, there are a number of possible approaches for modeling any single combustion event. Computationally expensive efforts have attempted to combine chemical kinetics with computational fluid dynamics (CFD) across multiple zones within a cylinder. However, the amount of time necessary to complete the routine (on the order of hours for a relatively fast computing system) is not practical for multiple cycles of simulation, especially when those cycles contain multiple combustion events. In addition, pressure wave dynamics, flame front propagation, quench, and knocking are all complex phenomena which compound the difficulty of combustion modeling. Thus, a model is chosen which sufficiently approximates the combustion event but does not encumber the total effort with excessive computational needs. The same principle is applied to each process that contributes to the operation of the SALEG.

This chapter provides a detailed description of the numeric model at the heart of the research and validates the model against examples and results found in current literature. The conceptualization of the linear engine under analysis is illustrated in Figure 1 with selected dimensions. The dual cylinder housing holds the tubular permanent magnet linear alternator (TPMLA) with mechanical compression springs or bounce chambers on either side of the alternator moving mass. Outside of the spring systems are the dual engine pistons and cylinders. Under the piston heads are crankcase spaces and bearing locations for the piston rods which rigidly connect the piston heads to the alternator moving mass. The crankcases allow for compression of the intake gas or mixture, depending on the choice of direct or port injection of fuel, and splash lubrication. Spark plugs in the cylinder heads might be included to provide assistance for low temperature $\mathrm{HCCl}$ combustion when necessary. Alternatively, glow plugs could replace the spark plugs to aid in combustion timing and control, or it may be found that neither are required for stable operation.

Just as in the fundamental analysis, Newton's second law (shown in Eq. 35) provides the basis for the translator dynamics. The sum of the forces acting on the translator is proportional to the moving mass and acceleration $(\ddot{x}=a)$. The forces acting on the translator are illustrated in the free body diagram (FBD) shown in Figure 20 along with the inertial response of the translator.

$$
\sum \vec{F}=m \ddot{x}
$$




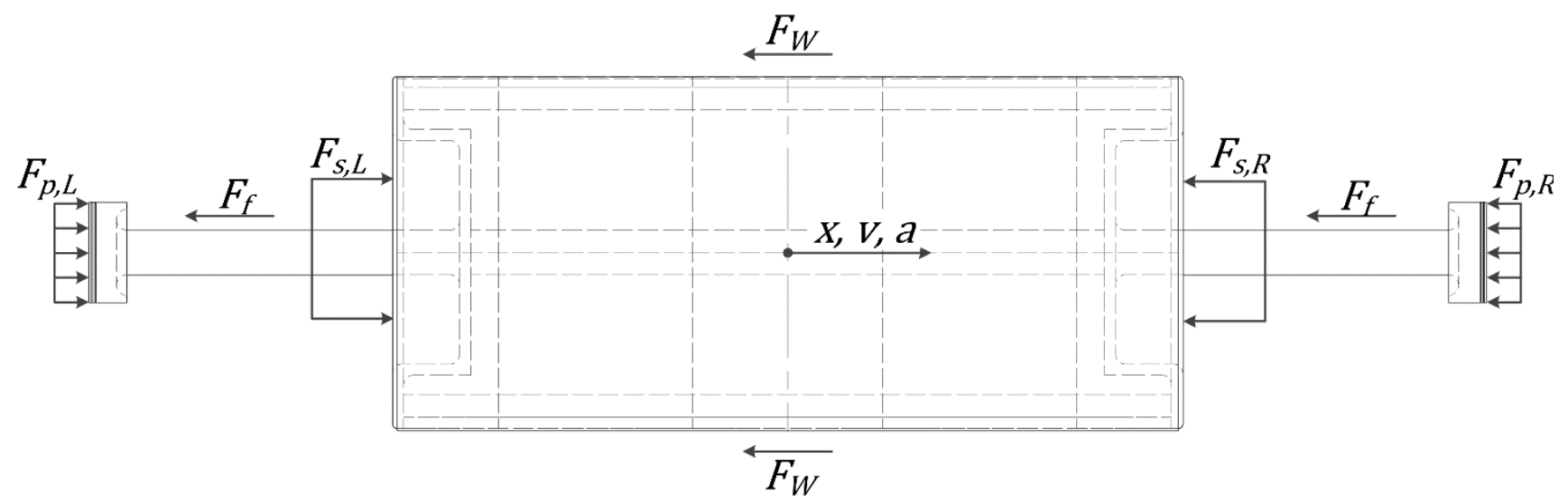

Figure 20: Free body diagram of SALEG translator with forces and inertia terms included.

In the FBD, $F_{p, L}$ and $F_{p, R}$ are the left and right forces associated with the engine cylinder pressures acting against the piston heads, $F_{S, L}$ and $F_{S, R}$ are the spring forces acting on the translator whether bounce chambers or compression springs, $F_{w}$ is the force from the linear electric machine, and $F_{f}$ is the friction acting against the motion of the translator at the bearing and piston ring locations. These forces cause the motion of the translator which is described by position $(x)$, velocity $(v=\dot{x})$, and acceleration $(a)$. Introducing these terms into Eq. 35 yields the dynamic relationship shown in Eq. 36.

$$
F_{p, L}-F_{p, R}+F_{s, L}-F_{s, R}-F_{w}-F_{f}=m \ddot{x}
$$

Eq. 36

To develop the model properly, each of the force terms deserves careful attention and so are described in the subsequent sections.

\subsection{Friction}

One of the anticipated benefits of FPLE technology is the reduction of friction as compared to conventional crankshaft engine technology. Because the linear engine directly drives the generation of electrical power rather than converting from linear kinetic energy (piston) to rotational kinetic energy (crankshaft), the number of components and contact surfaces is significantly reduced. According to researchers in [76] and corroborated in [77], 30\% of the fuel consumed in an engine goes to frictional losses. That is broken down into more specific losses: valve train and crankshaft friction can account for as much as $35 \%$ of the total friction lost; accessories (e.g. oil and water pump, A/C compressor, alternator) then account for $10-20 \%$; and the piston group losses range from $30-45 \%$ of the total friction. The FPLE married to the linear electric alternator eliminates the frictional losses associated with valve train, crankshaft, and accessories. Furthermore, piston group losses are expected to be reduced because of the lack of side thrust applied to piston head by the relatively lateral motion of the connecting rod in a 
crankshaft engine. Recent experimental results from DLR show total friction losses at 5.9\% [41] which is a significant reduction compared to crankshaft technology.

In any case, modeling of the friction force has taken four routes throughout investigations seen in the literature. A sensitivity analysis conducted in [78] shows that the shape of the friction force profile may be insignificant, but the available options are described for completeness. The trivial case is to ignore friction with the assumption that its affects are negligible [79]. However, the work at DLR and many others demonstrate that the amount of energy lost to friction is too significant to be ignored. The second approach for modeling friction is that of a constant force applied to the translator throughout its range of motion. Early correlations for this constant friction within a two-stroke engine are presented by Blair [80] and depend on the mean frictional pressure ( $\mathrm{fmep}$ ) as shown in Eq. 37 and have been applied in recent modeling investigations [35].

$$
\begin{gathered}
\frac{W_{f}}{V_{d}}=f m e p=A S f \\
V_{d}=\frac{\pi b^{2} S}{4} \\
W_{f}=2 F_{f} S
\end{gathered} \Rightarrow F_{f}=A \frac{\pi b^{2}}{8} S f
$$

In Eq. 37, $W_{f}$ is the frictional work, $V_{d}$ is the displaced volume, $S$ is the stroke length, $f$ is the engine speed or frequency as applicable, and $A$ is a constant $150 \mathrm{~kg} \cdot \mathrm{m}^{-2} \cdot \mathrm{s}^{-1}$ independent of system geometry. A third approach is the correlation presented by researchers from Sandia National Laboratories [12] - shown in Eq. 38.

$$
F_{f}=\operatorname{sign}(\dot{x})\left[f_{1}+f_{2}|\dot{x}|\right]
$$

In the correlation from SNL, model constants $f_{1}$ and $f_{2}$ were matched to experimental data gathered from a rapid compression machine used to test free piston technology with $\mathrm{HCCl}$ combustion. The model itself combines viscous damping effects proportional to the velocity of the translator and static friction associated with the force required to move the translator from rest. Unfortunately, the correlated constants are not reported. Finally, the friction has been modeled as only viscous damping (Eq. 39).

$$
F_{f}=c_{f} \dot{x}
$$

Eq. 39

Researchers from WVU correlated the model constant $\left(c_{f}\right)$ with experimental data gathered from a free piston linear engine and validated the model in ring-down testing [19], showing good success for the model. The same research suggested that the damping coefficient should be dependent on the piston bore and translator mass. Without more experimental data, such a correlation cannot be validated. Further, the translator mass effect may be less consequential than the stroke length based on the previously mentioned work by Blair. The viscous damping model is chosen for the present investigation 
because of its simplicity and proven effectiveness. The friction coefficient is calculated for a given model based on Blair's relationship for the average frictional pressure which has been shown to depend on engine geometry and operation. This relationship is given in Eq. 40

$$
c_{f}=A\left(\frac{\pi b^{2}}{8}\right) S_{\max }\left(60 f_{\text {avg }}\right)
$$

In this representation, the leading coefficient $(A)$ is tuned to corroborate with the frictional energy consumption ( $6 \%$ ) findings from DLR, $S_{\max }$ is the maximum stroke, and $f_{\text {avg }}$ is the average engine cycle speed in $\mathrm{Hz}$ scaled by 60 to match Blair's use of engine speed in revolutions per minute (rpm).

\subsection{Compression Springs}

For this investigation, the springs are modeled simply according to a constant stiffness $(k)$ and instantaneous deflection $(\delta)$ according to Eq. 41.

$$
F_{s}=k \delta
$$

Eq. 41

With the assumption of perfect, symmetric springs, the total spring force acting on the translator reduces to Eq. 42 which was demonstrated in the derivation of Eq. 6 and shows an independence of the combined spring effect with respect to geometry so long as symmetry is upheld.

$$
F_{S, L}-F_{S, R}=-2 k x
$$

While Eq. 42 represents a linear force profile for the combined spring effect, it is notable that compression springs do not necessarily have a linear profile and that compression springs do not have to be used at all. The use of regulated (compressed air) bounce chambers is being explored as an alternative, and may in fact offer a suitable means of controlling the SALEG through a wide range of operation.

\subsection{Alternator}

The primary objective of modeling the alternator is to find a relationship for the thrust force applied to the translator (notated as $F_{w}$ in Eq. 36) based on design parameters and instantaneous translator dynamics. Early analysis of a linear electric alternator by Boldea and Nasar [50,51] describes the general equations and practical design of single- and three- phase linear permanent magnet machines. Soon after, they continued their investigation of linear electric machines by demonstrating the use of an equivalent circuit to model a linear induction motor [54]. Previously at WVU, Cawthorne presented his dissertation on the modeling and optimization of a single-phase TPMLA where he employed finite element methods in ANSYS and an equivalent circuit to model the steady state solution of the device [62]. The process was extended to a three-phase device and validated against experimental data in [81, 
82]. The computationally expensive routine employed by Cawthorne is out of the scope of this investigations, but the use of an equivalent circuit continues to be seen in literature due to its computational simplicity.

Aside from the simplicity, the choice of the simplified equivalent circuit model is supported in another sensitivity study presented in [78]. In the study, it is shown that the simplified alternator force model tends to fit the mean value of the more complex model which includes the oscillating, position dependent flux pattern. Despite the apparently large variation in the force profile, the net energy effects on the translator dynamics are consistent between the two model choices.

Three recent publications demonstrate the use of the equivalent circuit model. The first [83], from Ulsan University in South Korea, represents the magnetic field strength with a Fourier series which enables the derivation of position dependent coil flux. Knowing the flux, the equivalent circuit equation can be manipulated to yield a relationship for the alternator thrust force dependent on design parameters and translator dynamics. At the heart of the resulting thrust force equation is a relationship which multiplies the translator velocity by a derived term that includes alternator geometry, equivalent circuit parameters, and translator position. In the second [35], researchers from the Beijing Institute of Technology in China and Newcastle University in the UK consider a simpler approach which multiplies the translator velocity by a load constant - in other words, the simplified thrust force is assumed to be proportional to the translator velocity by a given load constant. Their value for the load constant is given to be $100 \mathrm{~N} \cdot \mathrm{s} / \mathrm{m}$.

Finally, the third example of the equivalent circuit model for the alternator comes out of Shanghai Jiaotong University in Shanghai, China [84, 85]. Finite element analysis and simple equivalent circuit models were compared to demonstrate the validity of the simple model. Their solution involved a proportionality constant to relate the back EMF voltage to velocity which allowed the instantaneous current calculation via transfer function derived from the equivalent circuit. The thrust force was finally calculated based on its proportionality to the current and a second modeling constant. In any case, the simple model is shown to be effective and computationally cheap, but requires the use of modeling constants to accurately represent the nature of the alternator with respect to translator dynamics. Furthermore, these constants must be derived either from empirical data or computationally expensive analysis.

For the current investigation, the simplest of the models is chosen which relates the alternator thrust force to a single lumped parameter and translator velocity. The derivation of the model starts with the equivalent circuit as shown in Figure 21. 


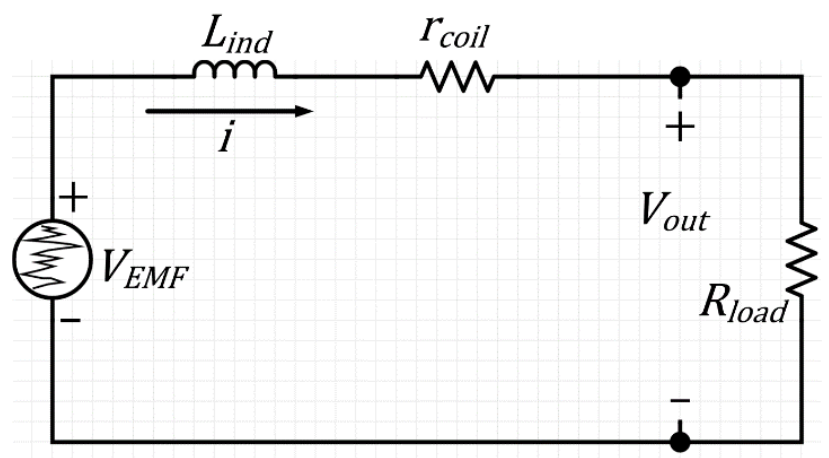

Figure 21: Equivalent circuit for alternator modeling - adapted from [62].

The equivalent circuit represents the average current flow through the alternator coils $(i)$ with an inductor $\left(L_{\text {ind }}\right)$ and coil resistance $\left(r_{\text {coil }}\right)$ along with the externally applied, resistive load $\left(R_{\text {load }}\right)$ [81]. The total circuit voltage equation set is given in Eq. 43.

$$
\begin{gathered}
V_{E M F}=L_{\text {ind }} \frac{d i}{d t}+i\left(r_{\text {coil }}+R_{\text {load }}\right) \\
V_{\text {out }}=i R_{\text {load }} \quad P_{\text {out }}=i^{2} R_{\text {load }}=i V_{\text {out }}
\end{gathered}
$$

The back EMF voltage $\left(V_{E M F}\right)$, output voltage $\left(V_{\text {out }}\right)$, and output power $\left(P_{\text {out }}\right)$ are all related to lumped alternator parameters. Now, Faraday's Law for Electromagnetics states that the EMF voltage is equal to the flux time derivative which can be expanded by the chain rule as shown in Eq. 44.

$$
V_{E M F}=-\frac{d \lambda}{d t}=-\left(\frac{d \lambda}{d x}\right) \dot{x}
$$

The total alternator flux $(\lambda)$ is assumed to be a function of translator position such that $d \lambda / d x=$ constant. This is shown to be approximately true in [79] where the end effects (flux profile around the limits of the translator stroke) show slight deviation from the assumed linear relationship. The force applied by the alternator on the translator is related to the power output through the velocity and the alternator efficiency $\left(\eta_{\text {alt }}\right)$ - given in Eq. 45.

$$
P_{\text {out }}=-\eta_{\text {alt }} F_{w} \dot{x}
$$

Assuming that the alternator efficiency encompasses the dissipation effects (inductor and resistor in the circuit) between the EMF and output voltage, the alternator force equation can be written as shown in Eq. 46.

$$
F_{w}=-\frac{i V_{E M F}}{\eta_{\text {alt }} \dot{x}}=\left(\frac{d \lambda / d x}{\eta_{\text {alt }}}\right) i
$$

Assuming again that the eventual load constant will compensate for dissipation, the current can be directly related to the load resistance and substitution with Eq. 44 yields Eq. 47. 


$$
F_{w}=-\left(\frac{d \lambda / d x}{\eta_{\text {alt }}}\right)\left(\frac{d \lambda / d x}{R_{\text {load }}}\right) \dot{x}=-\left(c_{A}\right) \dot{x}
$$

The alternator force equation describes the proportionality of the force to velocity through the load resistance and a load constant $\left(c_{A}\right)$ which lumps the flux profile and alternator efficiency together. The inverse relationship to load resistance illustrates that an increase of power output requires a decrease of load resistance, and the negative sign shown in Eq. 47 ensures that the alternator force always acts in the opposite direction of the translator velocity.

\subsection{Free Piston Engine Cylinders}

Perhaps the most important and complex of all the processes in the SALEG is that which occurs in each of the engine cylinders. The proposed dual piston linear engine operates as an alternating twostroke, port scavenged, methane fueled, $\mathrm{HCCl}$ combustion engine. The synergetic relationship between $\mathrm{HCCl}$ combustion and the variable compression free piston engine is well described by Lee [78]. Compression ratio rises and auto-ignition timing advances with respect to top dead center sympathetically. However, as start of combustion timing continues to advance, adverse work and heat transfer losses increase leading to reduced system energy and reduced compression. Conversely, as SOC timing recedes towards TDC, compression ratio tends to rise because the turn-around of the translator depends largely on cylinder pressure rise due to combustion. Capturing this and other dynamically interdependent relationships within the SALEG system can only be achieved with sufficient detail given to the engine modeling.

The two engine cylinders are modeled identically with 1-D cylinder pressure modeling techniques found throughout the free piston linear engine simulation literature. Use of a 1-D cylinder model sacrifices the greater accuracy that may be attained from a multi-zone approach which employs either chemical kinetics, computational fluid dynamics, or both to solve for cylinder pressure and temperature. However, such a method substantially increases the computational requirements for a simulation. It is the objective of this investigation to parametrically study the SALEG across a wide array of design possibilities, so model simplicity and accuracy must be balanced against sophistication and computational time.

The engine model is divided into four distinct processes: compression, combustion, expansion, and gas exchange. Cylinder gas leakage around the piston rings is neglected allowing for closed system analysis of the cylinder during compression, combustion, and expansion. While empirical correlations are available for each of these processes, they are often based on conventional engine technology and related through crankshaft position. As such, care must be taken to properly translate each expression to proper representation in the time domain. In addition, positional referencing such as that used in conventional engine technology (e.g. degrees after top dead center) has no real meaning for the free 
piston. Knowing the length of the stroke for a given cycle, it may be mathematically related to conventional terminology, but dead center positions are not fixed for the free piston and so cannot be used in the same manner.

With the assumption that gas leakage around the piston rings is negligible, compression, combustion, and expansion can be modeled as closed system processes. It is also assumed the cylinder gases behave according to the ideal gas law, so the cylinder pressure can be represented in the differential form given by Eq. 48 .

$$
\frac{d P}{d t}=\left(-\gamma \frac{P}{V}\right) \frac{d V}{d t}+\left(\frac{\gamma+1}{V}\right)\left(\frac{d Q_{h t}}{d t}+\frac{d Q_{H R}}{d t}\right)
$$

In Eq. 48, $\gamma$ is instantaneous specific heat ratio, $P$ is instantaneous cylinder pressure, $V$ is instantaneous cylinder volume, and $Q_{h t}$ and $Q_{H R}$ are heat transfer with the cylinder walls and heat release due to combustion respectively. For the ratio of specific heat, a $3^{\text {rd }}$ order polynomial fit is given in [86] which shows good correlation to $\mathrm{HCCl}$ engine data over a temperature range of $550 \mathrm{~K}-2150 \mathrm{~K}$. This relationship is provided in Eq. 49 and is correlated to temperature in units of Kelvin.

$$
\gamma=\left(-9.967 \cdot 10^{-12}\right) \cdot T^{3}+\left(6.207 \cdot 10^{-8}\right) \cdot T^{2}+\left(-1.436 \cdot 10^{-4}\right) \cdot T+1.396
$$

Cylinder heat transfer is modeled by convection and so depends on the instantaneous surface area within the cylinder $(A)$ and temperature difference between cylinder gas and cylinder wall ( $T$ and $T_{\text {wall }}$ respectively), as shown in Eq. 50.

$$
\frac{d Q_{h t}}{d t}=h A\left(T-T_{w a l l}\right)
$$

The wall temperature is assumed constant over the head, cylinder walls, and piston crown. The heat transfer coefficient $(h)$ can be calculated using various empirically correlated formulae, each with strengths and weaknesses according to application. Unfortunately, these empirical relationships are based upon slider-crank motion rather than the unusual and variable motion of the linear engine system. Piston velocity is the usual reference for the in-cylinder charge motion that enhances the heat transfer, along with instantaneous gas temperature, pressure, and cylinder volume. Hohenberg's formulation [87] has seen recent application in free piston engine modeling by Newcastle and Beijing researchers [34]. The relationship (Eq. 51) maintains the desired simplicity while including the terms which have proven to be most significant for cylinder heat transfer.

$$
h=a * V^{b} * P^{c} * T^{d} *(\bar{v}+1.4)^{e}
$$

Eq. 51

In the Hohenberg formulation, $V$ is volume in $\mathrm{m}^{3}, P$ is pressure in bar, $T$ is temperature in $\mathrm{K}$, and $\bar{v}$ is mean piston speed in $\mathrm{m} / \mathrm{s}$. In the original formulation, the empirical constants are: $a=130, b=-0.06$, 
$c=0.8, d=-0.4$, and $e=0.8$. However, the leading constant $(a)$ can be tuned to achieve a desired quantity of heat transfer per cycle. This feature is used to check simulation sensitivity to the selected heat transfer model in a later section.

Heat addition depends on the rate of combustion, which is readily programmed into a time-based model. However, empirical models such as those based on Wiebe functions [88, 89] were also originally derived from slider-crank engine data. There are several possible approaches for modeling any single combustion event. The current 1-D pressure model approach is considered single zone, as opposed to multi-zone methods which sacrifice simplicity and computation time for the potential of increased accuracy. These more extensive studies typically differ in overall objective and do not seek to achieve multiple cycles of operation for the simulated engine - see examples [90, 91].

In his dissertation [92], Mo concludes that combustion timing is the single most important variable for the entire combustion process and presents a series of relationships which sufficiently predicts 1-D $\mathrm{HCCl}$ engine performance. Unfortunately, these correlations are highly dependent on crankshaft position and cannot be adequately re-expressed for the free piston engine in the time domain. Such research indicates that single zone simulations can be satisfactory for accurate prediction of engine cylinder pressure during $\mathrm{HCCl}$ combustion operation, but such accuracy is dependent on proper correlation and application. The current work employs a time-based, single Wiebe function of the classic form [93].

$$
\chi(t)=1-\exp \left[-a\left(\frac{t-t_{S O C}}{C_{d}}\right)^{1+b}\right]
$$

In Eq. 52, the mass fraction burn $(\chi)$ is represented as a function of time, combustion timing $\left(t_{S O C}\right)$, combustion duration $\left(C_{d}\right)$, and shape parameters $a$ and $b$ equal to 5 and 2 respectively [34]. The same investigation also records a constant combustion duration of $5 \mathrm{~ms}$. Model sensitivity to the combustion profile and duration is demonstrated later in this chapter. With the mass fraction burn relationship, the heat release equation can be formulated.

$$
\begin{aligned}
& \frac{d Q_{H R}}{d t}=\eta_{\text {comb }} E_{\text {fuel }} \frac{d \chi(t)}{d t} \\
& \frac{d Q_{H R}}{d t}=\eta_{\text {comb }} E_{\text {fuel }}\left[a \frac{b+1}{C_{d}}\left(\frac{t-t_{S O C}}{C_{d}}\right)^{b} \exp \left(-a\left(\frac{t-t_{\text {SOC }}}{C_{d}}\right)^{1+b}\right)\right]
\end{aligned}
$$

In Eq. 53, the heat release rate is dependent on the mass fraction burn rate and the total heat released which is the product of combustion efficiency $\left(\eta_{\text {comb }}\right)$ and total fuel energy $\left(E_{f u e l}\right)$. The fuel energy is calculated as the product of total fuel mass in the cylinder and the lower heating value (LHV) of the fuel. It is noted here that while a LHV of $48 \mathrm{MJ} / \mathrm{kg}$ is assumed for this investigation, the energy content of the fuel is somewhat arbitrary given the number of other parameters which must be dealt with. In fact, fuel energy changes can be achieved by either varying the LHV or the fuel mass (akin to adjusting 
the equivalence ratio), and any single combustion event is additionally dependent on combustion efficiency which is out of reach for this investigation. On the other hand, fuel properties in general can have significant impact on combustion performance and a range of fuels have been examined for $\mathrm{HCCl}$ combustion [94-96]. The given LHV, along with stoichiometric air-to-fuel ratio of 17.5, is meant to be representative of methane - a relatively high energy content, gaseous fuel with simple molecular structure. Meanwhile, the combustion efficiency is assumed to be $95 \%$, but as Mo demonstrates in [92], efficiency depends on operating conditions such as engine speed, combustion timing, equivalence ratio, and wall temperature. Mo finds a correlation for efficiency based on crankshaft driven engine data, but no such data yet exists for the free piston engine.

For $\mathrm{HCCl}$ combustion, the ignition timing is independent of an external trigger (such as spark or injection) but instead depends on chemical kinetics: temperature, pressure, mixture composition, and stratification [65]. Because the employment of a sophisticated combustion prediction routine is outside the scope of this investigation, the ignition timing is predicted with the use of a knock integral. The general form for the knock integral is given in Eq. 54 .

$$
\int_{0}^{t_{S O C}} \frac{1}{\tau} d t=1.0
$$

When the ports close and compression begins, the integration is initiated. The combustion ignition timing (start of combustion, $t_{S O C}$ ) is found when the integral of $1 / \tau$ equals unity where $\tau$ is a correlated relationship that involves the effects of fuel mixture, temperature, and pressure. Two notable relationships for $\tau$ are available in the literature. The first of these is described in [97] and is validated against data from a Cooperative Fuel Research (CFR) engine fueled by iso-octane and operating on $\mathrm{HCCl}$ combustion. The relationship is provided in Eq. 55

$$
\tau=A \omega \phi^{-x} \exp \left(\frac{b}{T} P^{n}\right)
$$

In Eq. 55, $A, b, n$, and $x$ are model parameters with base values of $9.291 \cdot 10^{-7}, 16909,-0.1121$, and -0.688 , respectively. Meanwhile, $\omega$ is engine speed in revolutions per minute which is equivalent to frequency in units of $\mathrm{min}^{-1}$ for the two-stroke free piston engine, $\phi$ is equivalence ratio, $T$ is gas temperature $(\mathrm{K})$, and $P$ is cylinder pressure in $(\mathrm{kPa})$. The second of the two relationships correlates autoignition of iso-octane from a rapid compression machine (RCM) [98] according to the relationship for $\tau$ given in Eq. 56.

$$
\tau=1.3 \cdot 10^{-7} \cdot P^{-1.05} \cdot \phi_{F O}^{-0.77} \cdot \chi_{O_{2}}^{-1.41} \cdot \exp \left(\frac{E}{R \cdot T}\right)
$$


Similarities between the two ignition timing models are evident by the use of pressure ( $P$ in atm), temperature ( $T$ in $\mathrm{K}$ ), and mixture composition. However, mixture composition is given as the product of effects from equivalence ratio $(\phi)$ and oxygen mole fraction $\left(\chi_{\mathrm{O}_{2}}\right.$ in \%) while $R$ is the universal gas constant $(\mathrm{J} / \mathrm{mol}-\mathrm{K})$ and $E$ is activation energy (33.7 kcal). This model was employed by Lee in recent free piston engine modeling efforts [78], and both are used in the current investigation for the sake of comparison.

The final engine cycle process to be described is the gas exchange. For the ported two stroke engine, cylinder recharge is accomplished as the motion of the piston head around bottom dead center enables the flow of exhaust out and fresh charge into the cylinder. It is a complex process dependent on many factors including pressure, temperature, mixture stratification, port geometry, intake conditions, and engine speed. For the free piston engine, the complexity is exacerbated because stroke length, bottom dead center position, and engine speed can vary with each cycle. Throughout the modeling literature, a common practice is to simplify the problem by assuming an instantaneous process which results in immediate replacement of exhaust gas with intake mixture at intake pressure and temperature. While experimental data is still rare, the technique is validated by Newcastle and Beijing researchers in [34] and by South Korean researchers in [83]. Thus, the simplification is assumed to be reasonable and is applied to the current study. The cylinder mass is divided between three types: burnt mixture, fuel, and ideal air $\left(21 \% \mathrm{O}_{2}\right.$ and $79 \% \mathrm{~N}_{2}$ by volume). The relationship is given in Eq. 57 .

$$
m_{\text {gas }}=m_{\text {burnt }}+m_{\text {fuel }}+m_{\text {air }}
$$

Eq. 57

The total mass inside the closed cylinder remains constant at all times, but the amounts of burnt mixture, fuel, and air vary throughout each engine cycle. If the piston does not reach a position which enables the gas exchange process, the burnt mixture is not replaced by fuel and air. During combustion, the fuel and air are converted to burnt mixture according to the mass fraction burn rate and combustion efficiency. The burnt mixture is assumed to be entirely inert, and the equivalence ratio determines the amount of fuel and air to add to the cylinder during the gas exchange process. Additionally, the cylinder mixture is retained at a specified EGR rate. For each cycle where gas exchange is achieved, amounts of burnt mixture, fuel, and air are all retained in the cylinder according to the EGR rate and fuel and air are added so that the total fuel and air in the cylinder satisfy the specified equivalence ratio. In later simulations, fueling is controlled by affecting the equivalence ratio to cause either increase or decrease in the amount of fuel.

\subsection{Simulink Implementation}

Simulink and MATLAB ${ }^{\circledR}$ offer a powerful set of tools to model and simulate a wide variety of systems. For this investigation, special attention is given to modularity, or component based modeling, 
so that the model can be passed on to future researchers who might desire to incorporate alternative and possibly more sophisticated sub-models for the various processes. In particular, experimental efforts at WVU may eventually result in free piston engine correlations that would greatly increase the accuracy of the virtual model. Moreover, the Simulink model serves as a convenient platform to perform parametric sweeps and testing of various control architectures. This section details the basic architecture of the model beginning at the highest level, shown in Figure 22. At the master level, the main feedback loop is evident as system bus signals are gathered and fed back into the three primary model partitions.

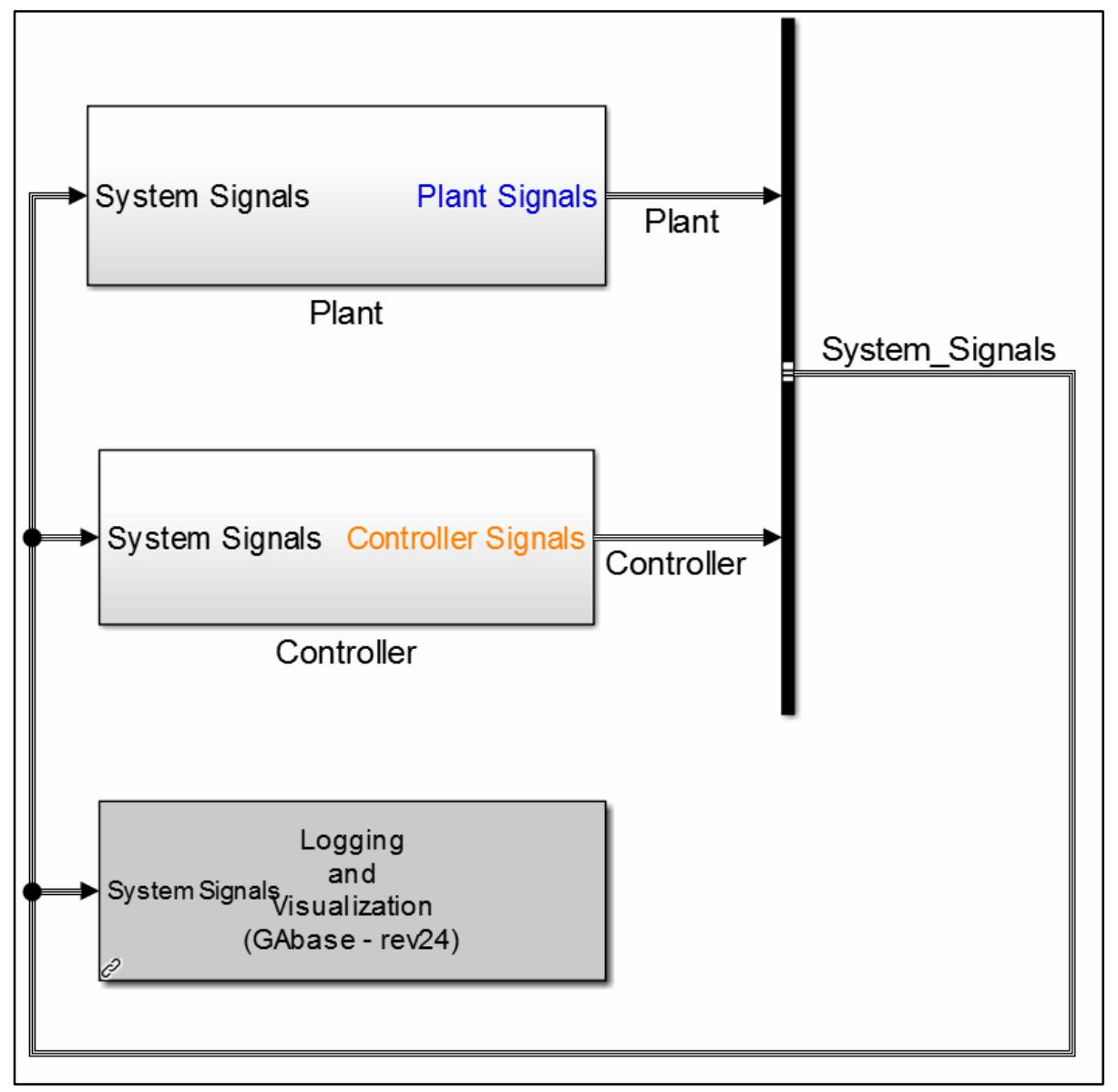

Figure 22: Master level of the Simulink model.

One of the Simulink tools utilized is that of linked library blocks. These are built and stored in an external Simulink file. The use of a Simulink library provides a source for reusable Simulink subsystems and helps to facilitate modeling consistency and reduced simulation time (via treatment of some library blocks as atomic units). In Figure 22, the 'Logging and Visualization' block has a dark gray background to signify that it is a library block. Throughout the Simulink model, all library blocks are designated with the dark gray background. Also, color coding is applied throughout the model to help maintain organization of signal routing. 'Plant' signals are given in blue and 'Controller' signals are in orange. 
The 'Logging and Visualization' subsystem provides no information to the simulation, but stores the data in the MATLAB ${ }^{\circledR}$ workspace for post-processing. The 'Controller' block is developed in greater detail later in Ch. 5 of this dissertation. The primary focus of this section is on the 'Plant' system which calculates all of the forces acting on the translator according to the previously given relationships and solves for the translator dynamic response. Figure 23 shows the layout of the 'Plant' with signal organization evident.

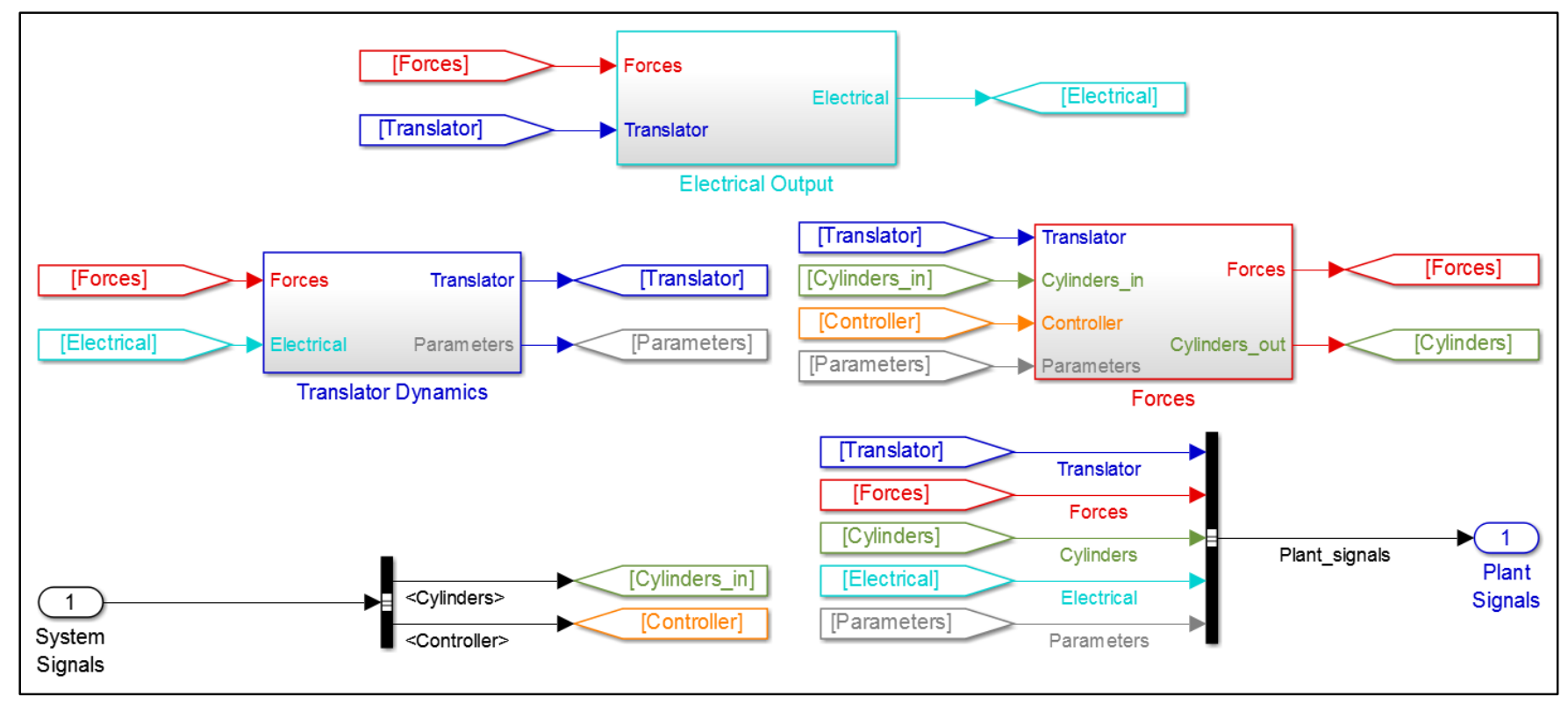

Figure 23: 'Plant' subsystem of the Simulink model.

The 'Plant' system is composed of three subsystems: 'Electrical Output,' 'Translator Dynamics,' and 'Forces.' The 'Electrical Output' subsystem calculates the power output of the 'Plant' via the product of alternator force and translator velocity with an assumed alternator efficiency of $95 \%$ - a fixed value throughout this investigation. The subsystem is shown in Figure 24.

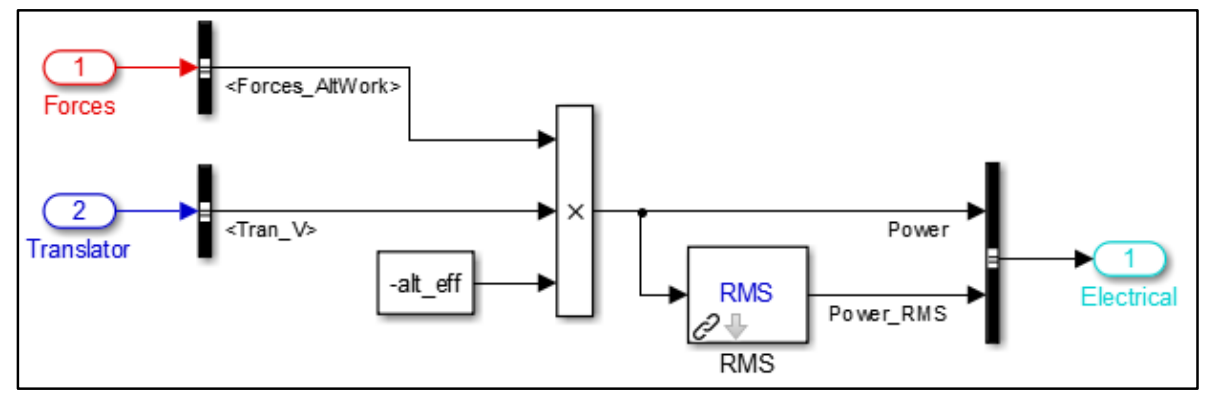

Figure 24: 'Electrical output' subsystem of the Simulink model.

Because the power output of the 'Plant' oscillates in sympathy with the translator velocity, Root Mean Squared (RMS) power is calculated as an additional output. Alternator efficiency is shown with a 
negative to convert the energy lost from the system (a negative value) to positive power output. The 'Translator Dynamic' subsystem is shown in Figure 25.

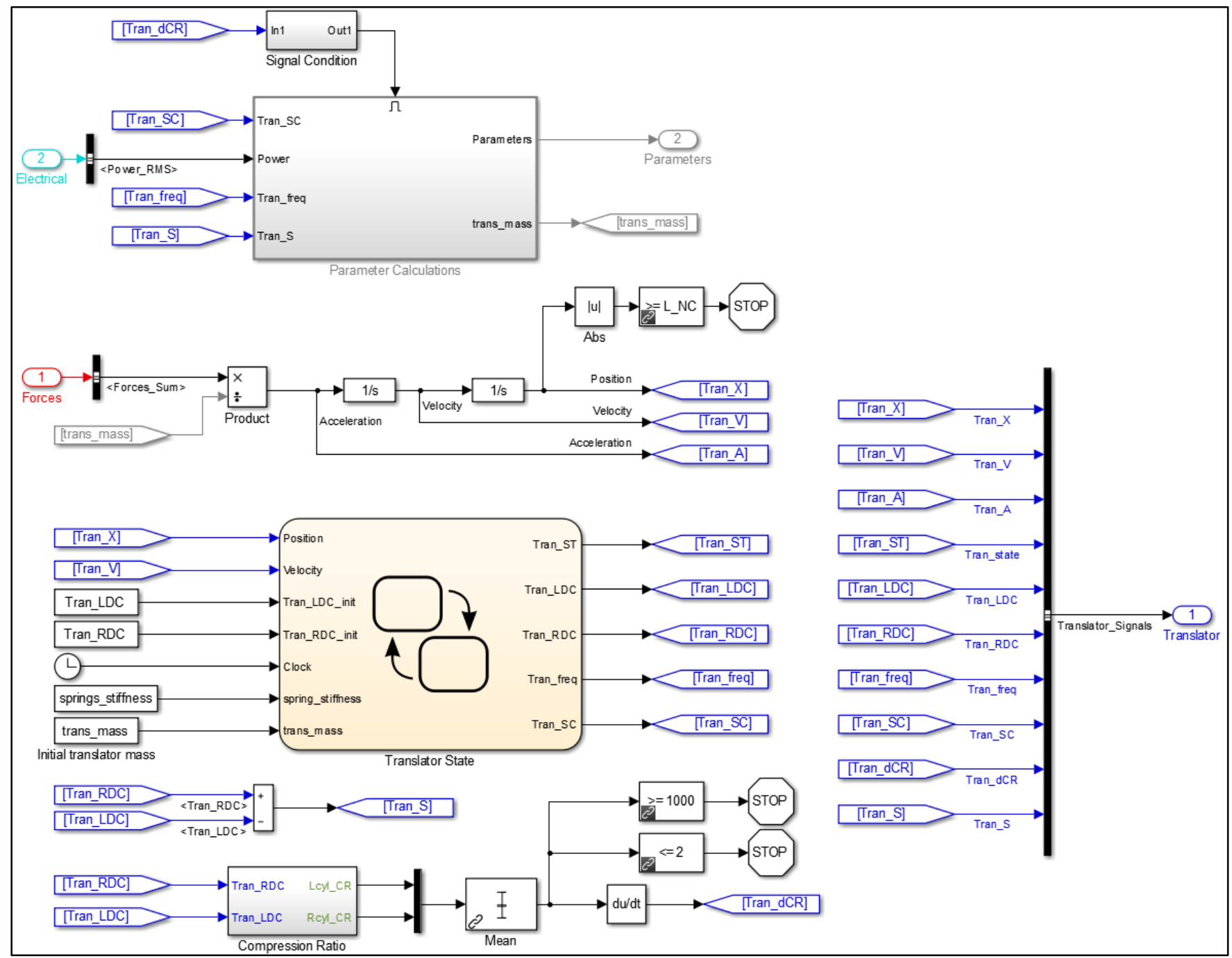

Figure 25: 'Translator Dynamics' subsystem of the Simulink model.

The primary function of the 'Translator Dynamics' subsystem is to calculate the translator position, velocity, and acceleration based on Newton's second law (discussed previously). Additional calculations are seen in Figure 25 which enable various model functions such as simulation stop conditions in cases where a solution grows unstable (e.g. engine ring-up or ring-down). The 'Translator State' flow diagram enables calculations for translator frequency, stroke length, dead center positions, and mean change in compression ratio based on the state of the translator motion. The 'Parameter Calculations' subsystem will be discussed in more detail in a later section. For this particular Simulink model which focuses only on steady state performance, it enables an iterative component design routine for translator mass and system geometry. The 'Parameter Calculations' routine is not included in a separate Simulink model which assumes all system parameters are known and is used to predict transient behavior. 
Figure 26 shows the Forces subsystem which is divided between the four main force groups: 'Alternator,' 'Friction,' 'Springs,' and 'Cylinder Pressures.' Once the forces are calculated, they are summed and passed to the common bus to be distributed with the rest of the system signals as necessary. The appropriate sign (negative or positive as required by the free body diagram in Figure 20) is applied within each of the subsystems.

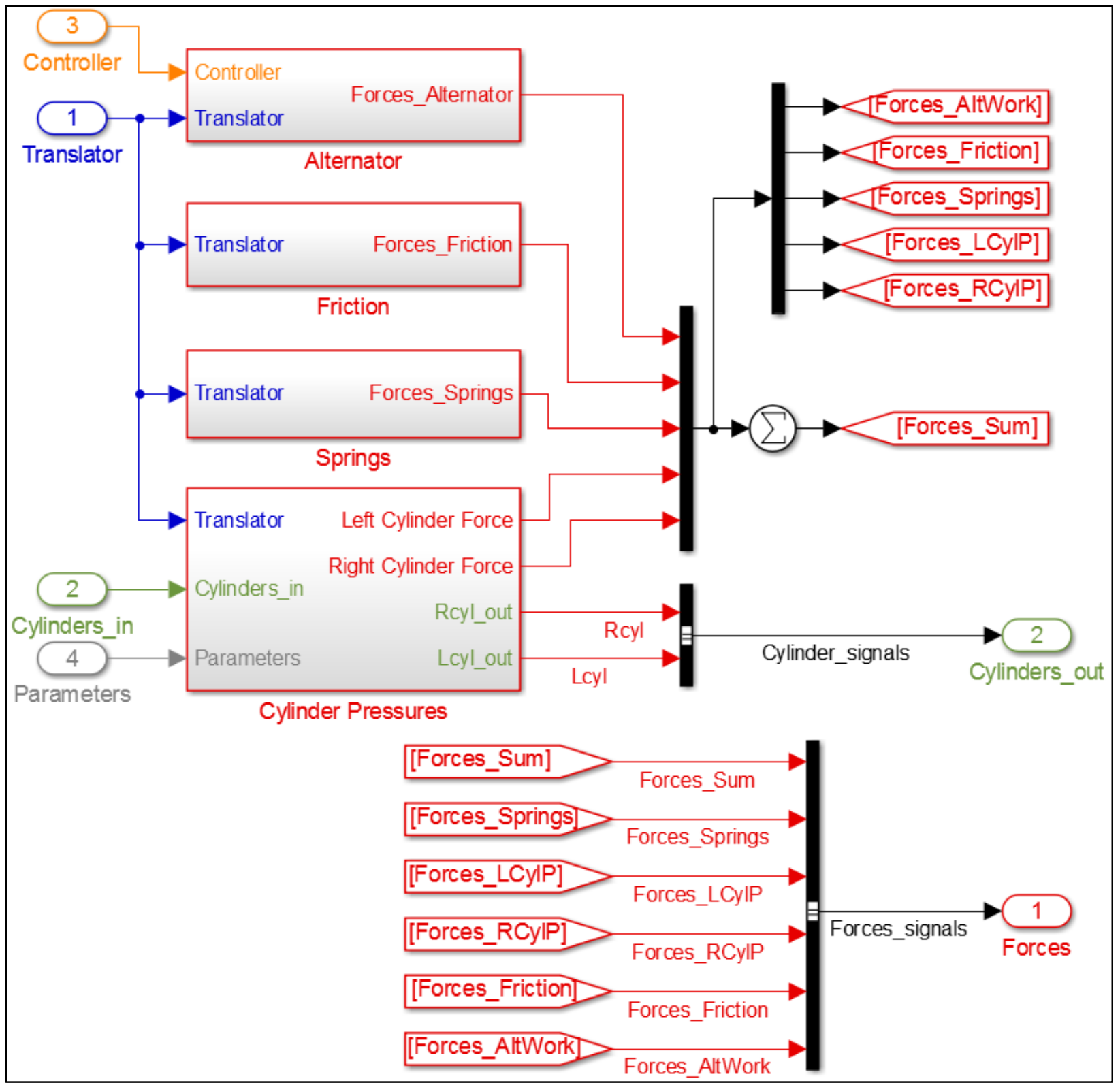

Figure 26: 'Forces' subsystem of the Simulink model.

The 'Alternator' subsystem calculates the force exerted by the alternator on the translator as a function of translator velocity and a load constant. This is shown in Figure 27. Also evident is the effect of the controller which encourages the stability of the system by increasing or decreasing the load 
constant of the alternator depending on the change in stroke length of the translator from stroke to stroke. The effects of this controller are demonstrated in the next section covering model sensitivity.

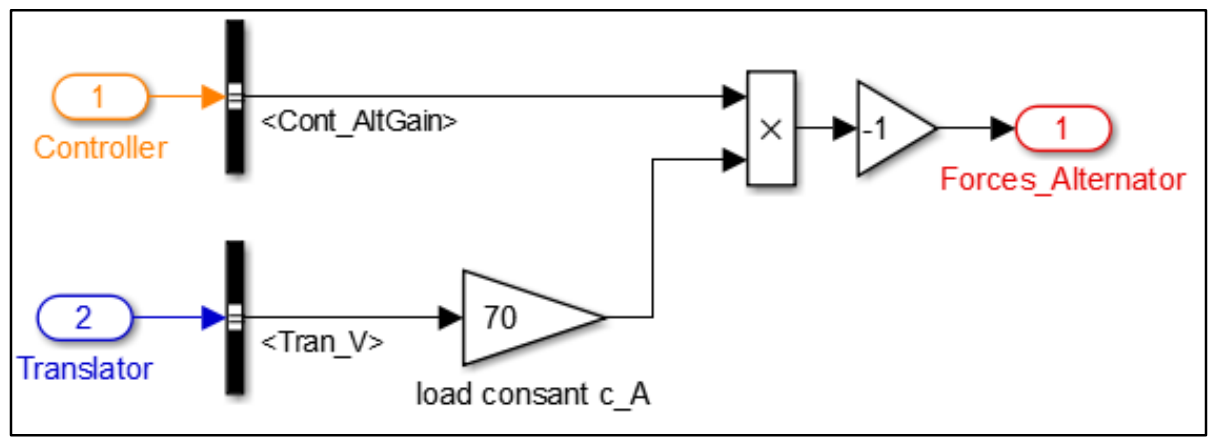

Figure 27: 'Alternator' subsystem of the Simulink model.

The friction model as described in Eq. 40 is shown in Figure 28.

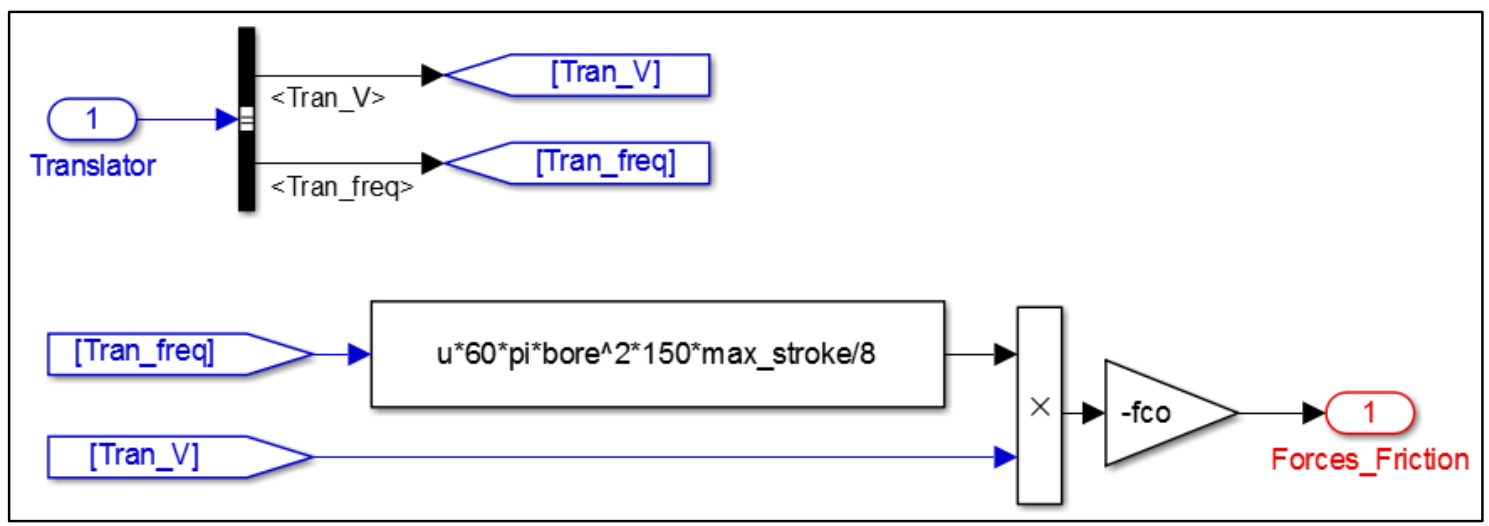

Figure 28: 'Friction' subsystem of the Simulink model.

The friction model is scaled by a friction factor (fco) to tune the frictional losses according to the loss analysis given by DLR in [41]. The two, symmetric ideal compression springs exert a force on the translator according to Eq. 42. The subsystem for the springs is shown in Figure 29.

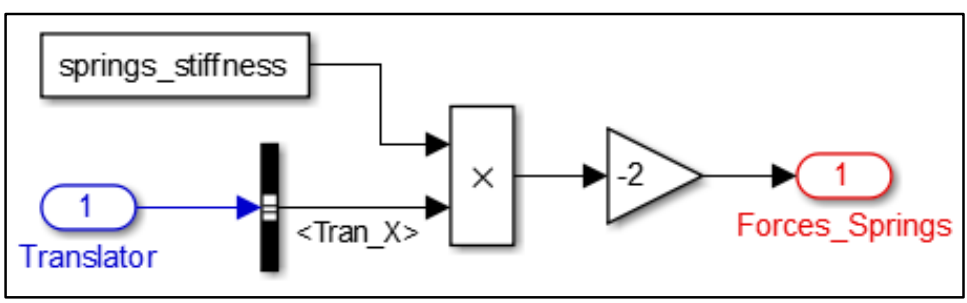

Figure 29: 'Springs' subsystem of the Simulink model.

The most complicated force models are those that involve the engine cylinder pressures. Because of the similarity between the two cylinders, only the right cylinder subsystem is described in this section. Figures describing the left cylinder can be found in Appendix A. For the right cylinder, Figure 30 shows the overall structure of the cylinder model separated between elements that are unique to the right cylinder - calculations for volume and compression ratio, the state flow subsystem, initial conditions, and 
signal storage - and a library block which is common for each of the cylinders and is primarily responsible for cylinder pressure calculation given spatial and temporal changes with each step in the solution.

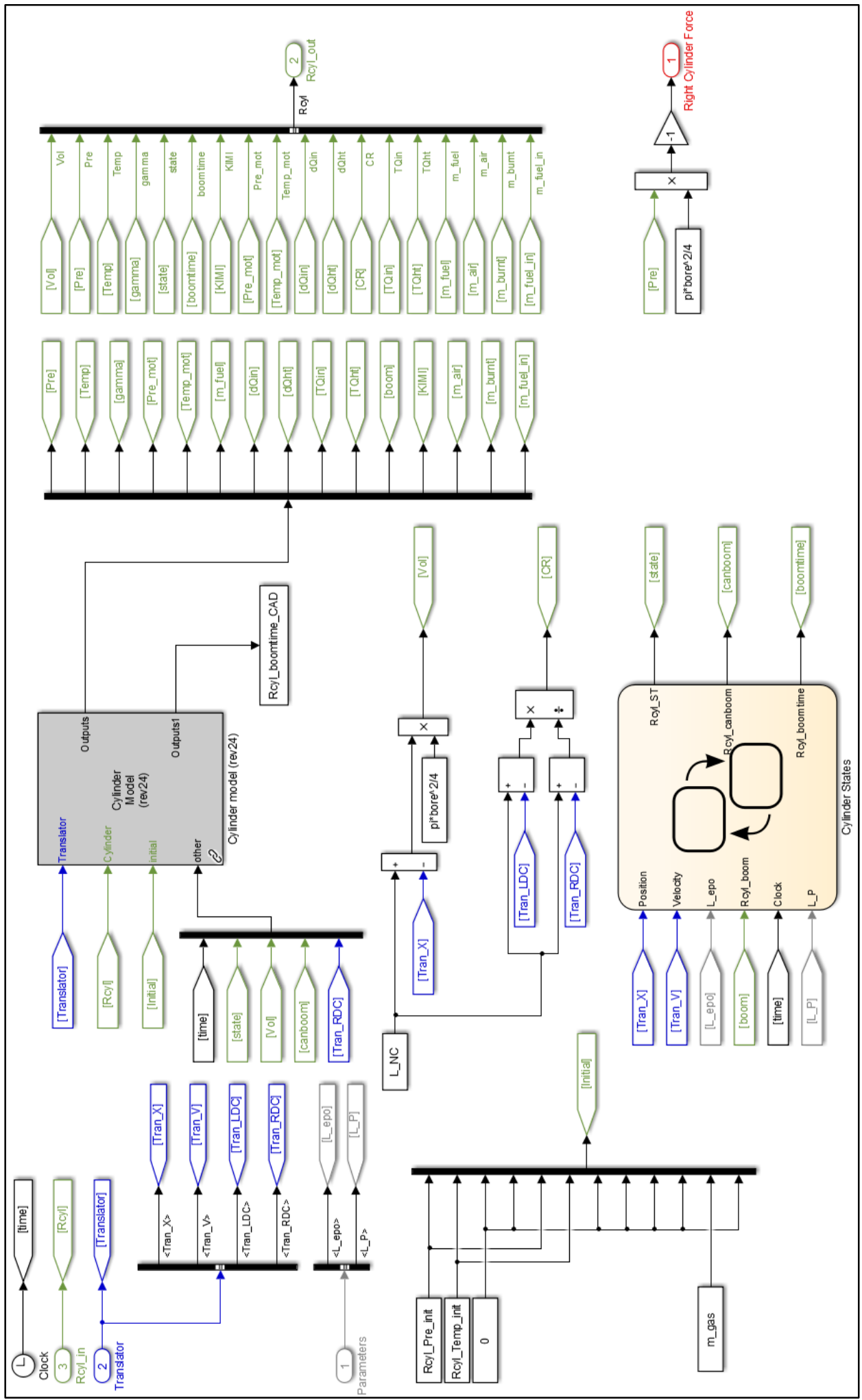

Figure 30: 'Right_Cylinder' subsystem of the Simulink model. 
The cylinder state flow diagram (given in Figure 31) is used to manage the cylinder processes. The ports opening or closing is dependent on position and begins or ends the gas exchange process. If gas exchange does not occur, in other words the piston head does not reach a bottom dead center position which opens the ports, combustion is not enabled during the subsequent compression stroke which begins as soon as the expansion stroke ends. This condition is generally indicative of an unstable system and an eventual ring-down of the translator. Combustion can end during either the compression or expansion stroke, a condition dependent on the translator velocity.

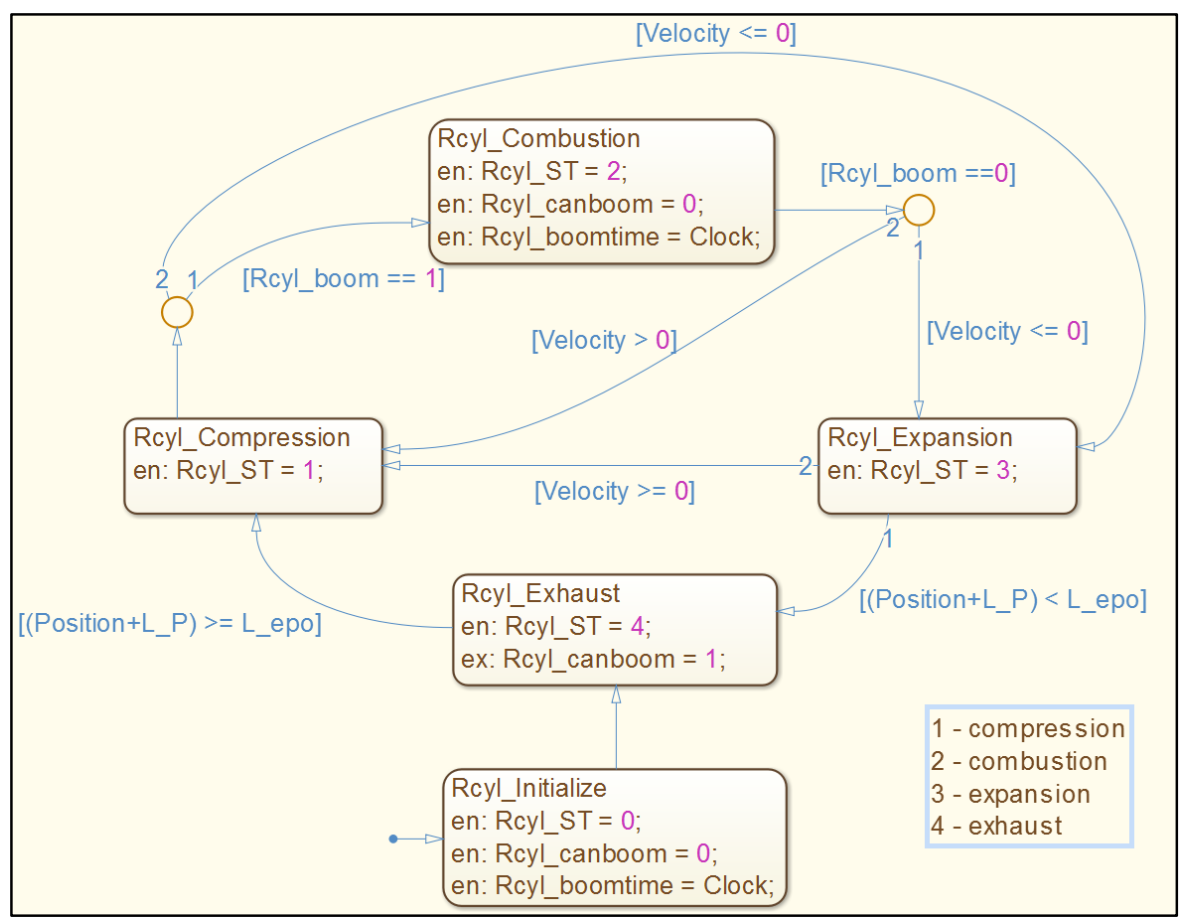

Figure 31: 'Cylinder States' subsystem of the Simulink model.

The library block 'Cylinder model' is common between the two engine cylinders and has undergone the greatest number of revisions throughout the investigation. The 'Cylinder model' takes the complete set of state values for the cylinder and computes the cylinder state for the next time step based on the relationships described in section 3.4 of this dissertation. 


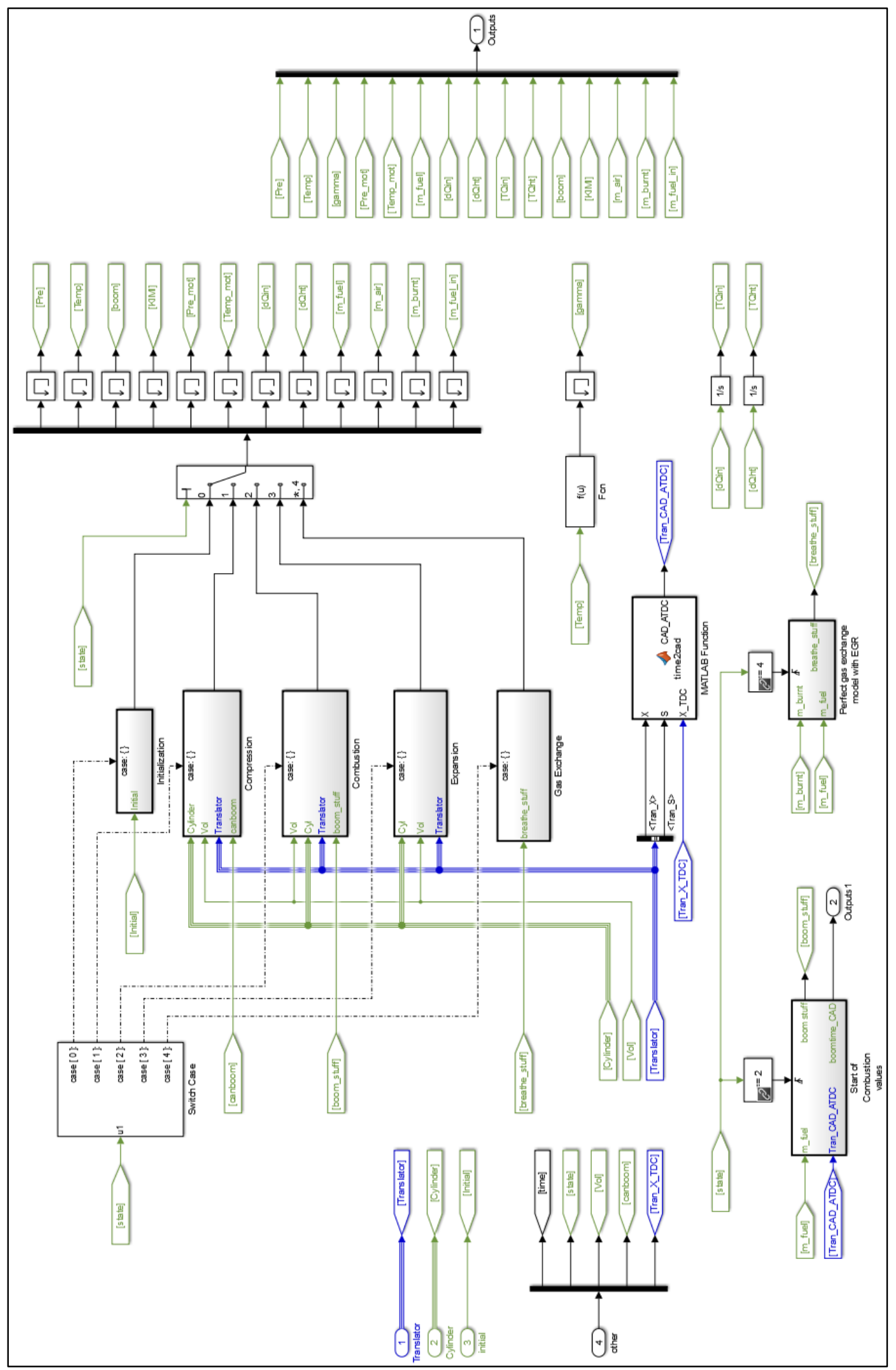

Figure 32: 'Cylinder model' subsystem of the Simulink model. 
The four distinct cylinder processes are evident in Figure 32 along with calculations for process parameters that vary from cycle to cycle. The 'Start of Combustion values' subsystem computes combustion parameters such as the amount of fuel energy to be released during the combustion event. The 'Perfect gas exchange model with EGR' subsystem calculates the amount of fuel to be added to the cylinder during the gas exchange process. Later, a controller signal is supplied to this subsystem to increase or decrease the fueling. The 'Initialization' subsystem passes the initial conditions directly through for the first time step in the simulation. The 'Compression' subsystem is shown in Figure 33.

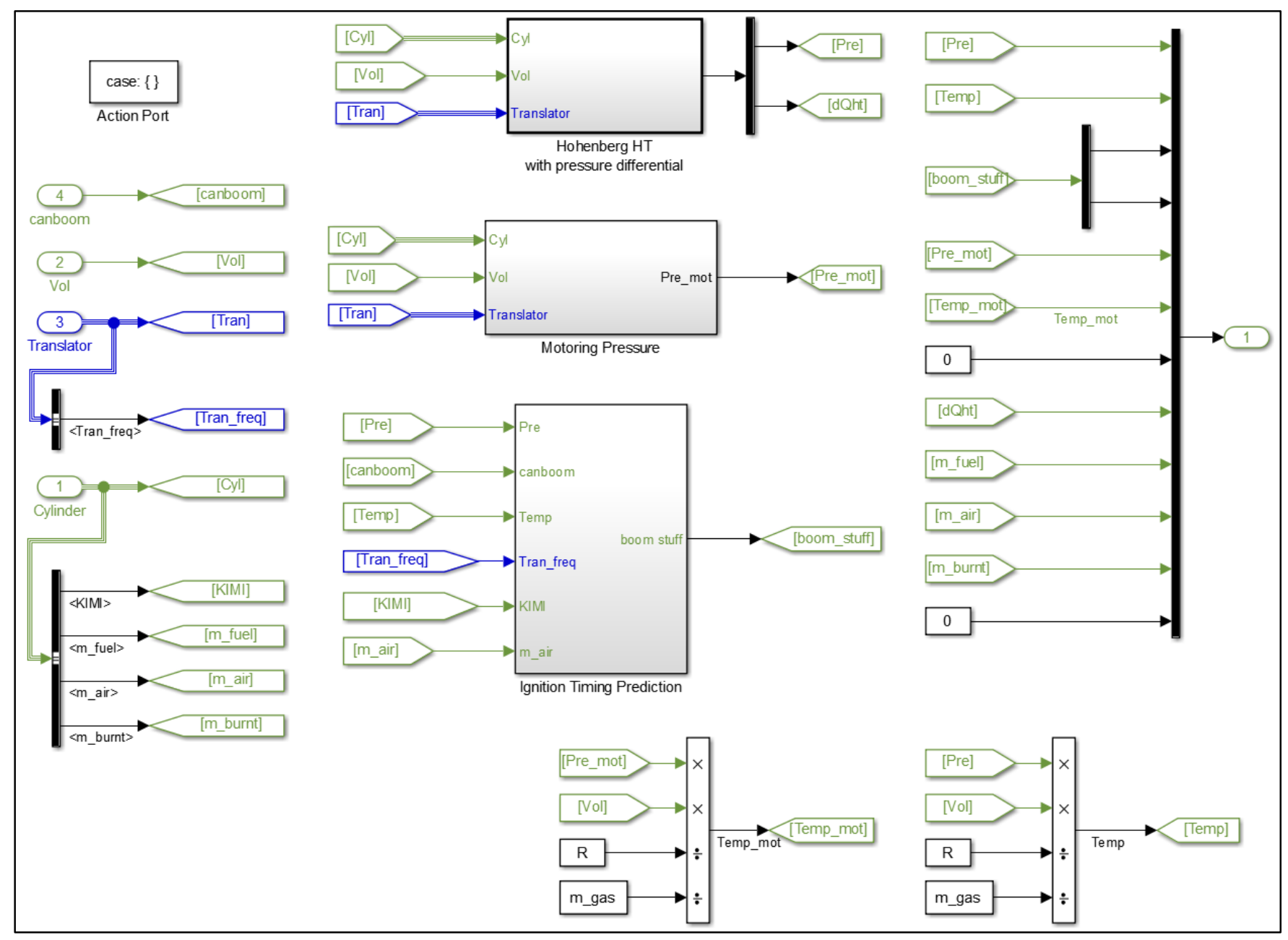

Figure 33: 'Compression' subsystem of the Simulink model.

The compression process is divided into four sets of calculations. The cylinder pressure increases according to the pressure differential and Hohenberg heat transfer relationships described in Eq. 48 and Eq. 51, respectively. The motoring pressure, which is useful in its own right for post-processing analysis and must be supplied for certain heat transfer correlations, is calculated in the same fashion as the actual cylinder pressure. However, after the end of combustion, the cylinder state can return to compression and the separation between motoring and actual pressure profiles requires distinct calculations. The 
'Ignition Timing Prediction' subsystem computes the knock integral according to the $\mathrm{CFR} \mathrm{HCCl}$ engine correlation in Eq. 55 (or RCM ignition model if selected) and provides the trigger which changes the cylinder state to the combustion process. The knock integral integration is reset each cycle at the start of the compression process. Finally, actual and motoring temperatures are calculated based on the ideal gas relationship and the assumed ideal gas constant for air.

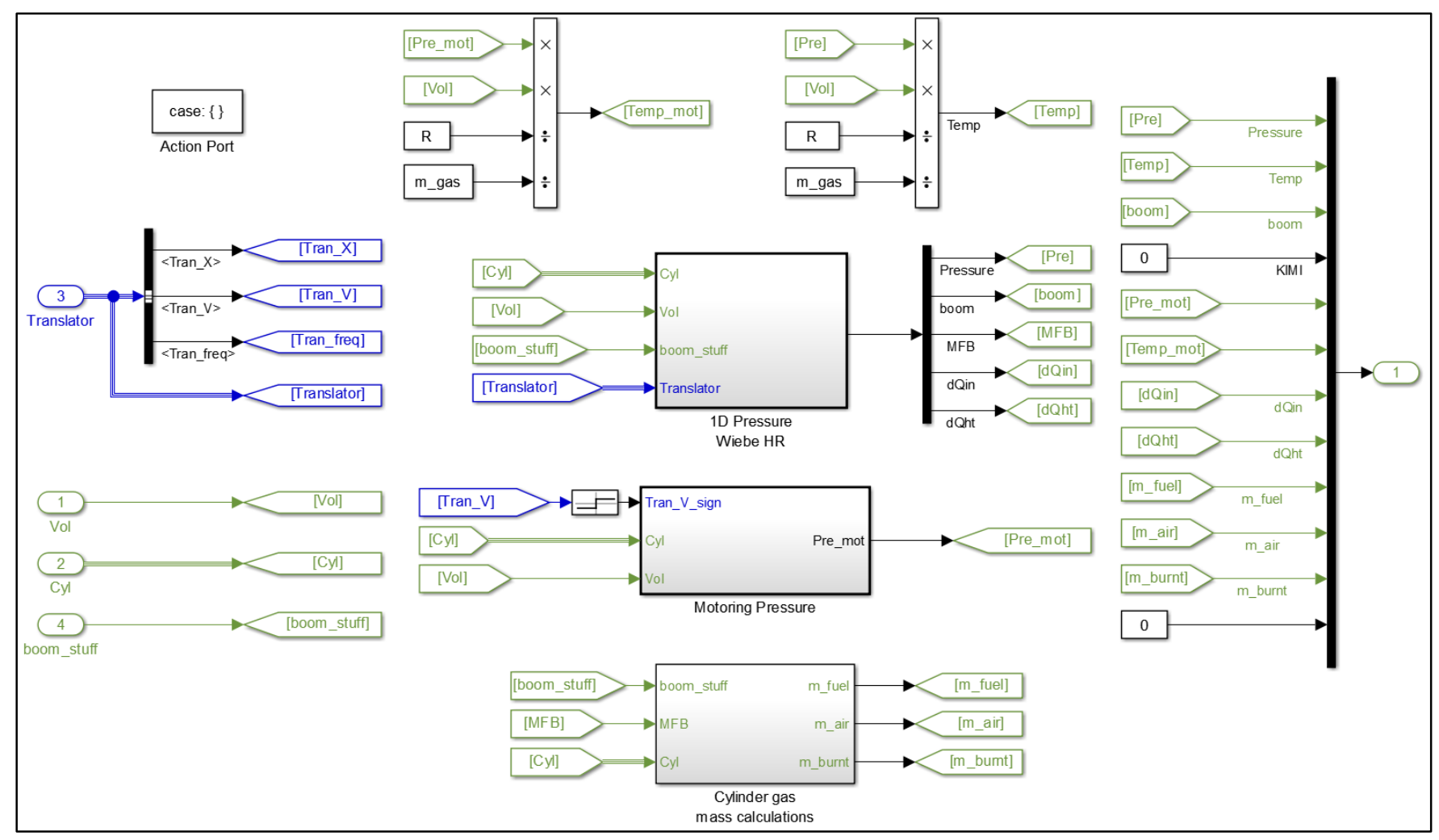

Figure 34: ‘Combustion’ subsystem of the Simulink model.

Figure 34 shows the 'Combustion' subsystem with four sets of calculations. Depending on pressure calculations and the assumed gas properties, actual and motoring temperatures are calculated. Motoring pressure is calculated with the Hohenberg heat transfer model and pressure differential. The '1D Pressure Wiebe HR' subsystem calculates the heat released during combustion and heat transfer to the cylinder wall for the given step in time and provides the mass fraction burnt as a percentage of the total fuel that will combust during the cycle (see Figure 35 for detail). In the 'Cylinder gas mass calculations' subsystem, the mass fraction burnt percentage is used to calculate the distribution of the mass within the cylinder, decreasing the amount of fuel and air while increasing the amount of burnt gas. The total amount of mass within the cylinder remains constant according to mass conservation and the assumption of no leakage. 


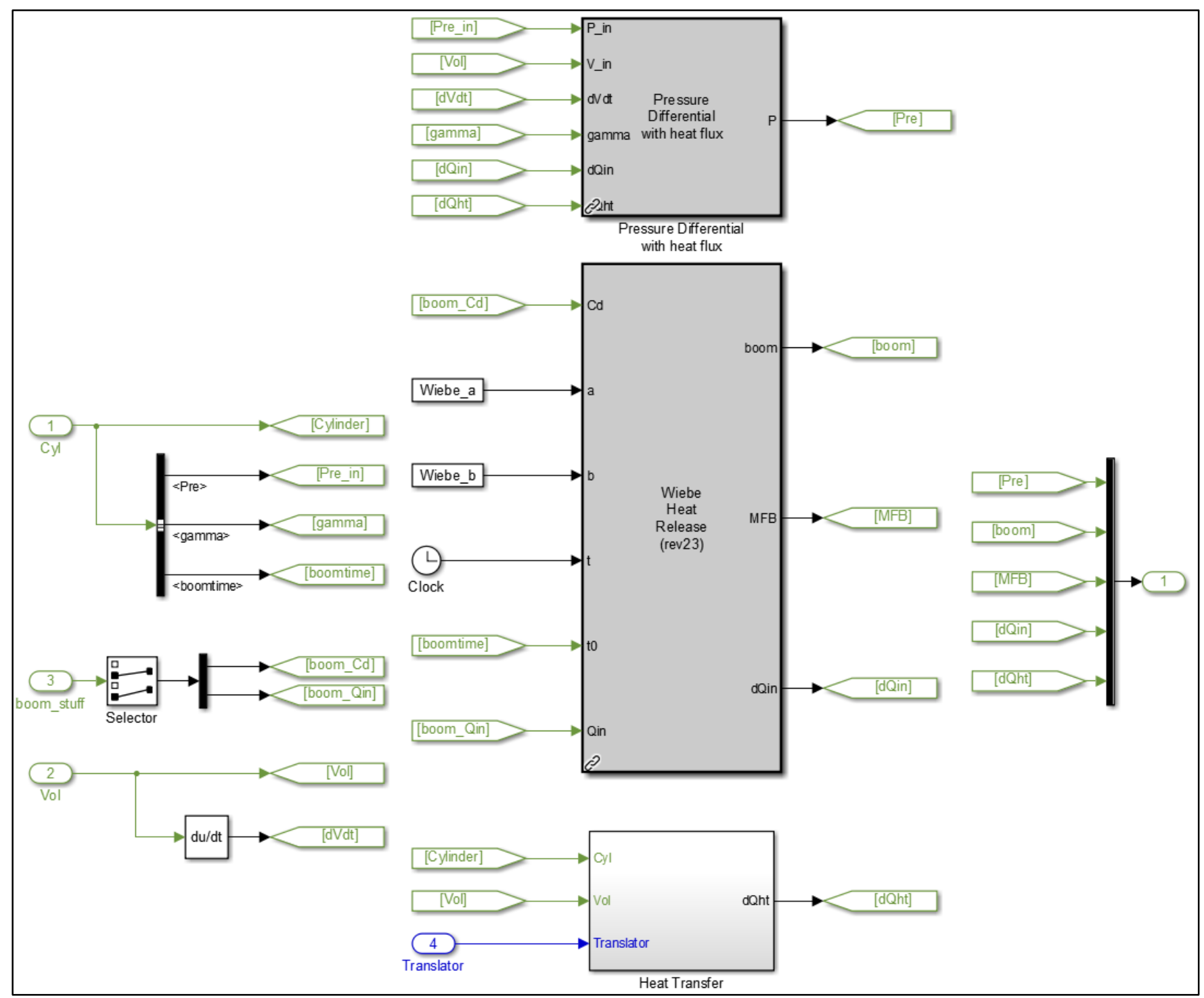

Figure 35: '1D Pressure Wiebe HR' subsystem of the Simulink model.

The expansion process follows the same set of calculations as the compression process, except that the knock integration is not computed. For this simplified 1-D pressure model, it is assumed that $\mathrm{HCCl}$ combustion ignition can only occur during the compression process. The gas exchange process is greatly simplified compared to reality so that the pressure and temperature immediately match intake conditions. The mass calculations then follow Eq. 57 and the corresponding description.

To summarize, the simulation is constructed as a large feedback loop. The position of the translator, conditions in the engine cylinders, loading from the alternator and friction, and translator inertia result in the instantaneous net acceleration. Integrating the acceleration then provides velocity and position of the translator for the next step in time. These values are then used to compute the forces on the translator at the next time step. The process continues until a stop time condition is reached - for example, the 
simulation may be constrained to stop after 3.5 seconds is reached within the simulation space. The complexity of the model stems from the sub-models used to calculate the forces. Specifically, the engine cylinder models consist of numerous calculations related to combustion, heat loss, thermodynamic behavior, and mass flow, even for the relatively simple single zone pressure modeling approach presented. While improvement of the cylinder modeling (and other processes) would greatly benefit the accuracy, simulation of multiple cycles requires greater computation time and limits the model sophistication. The next sections test the given model and its sub-models to demonstrate its precision and repeatability.

\subsection{Model Sensitivity and Validation}

Because this investigation is entirely dependent on the numeric model, it is paramount to verify the prediction capabilities of the simulation. Experimental data is unavailable for direct corroboration, so validation is achieved through a combination of solution method sensitivity testing and comparison to results and trends seen in the literature.

\subsubsection{Base Case}

To facilitate the validation process, a base case is provided. In addition to the Simulink model already described, tuning mechanisms are included to achieve reasonable steady state operation. These tuning mechanisms apply to: the physical parameters by affecting system geometry and translator mass depending on device performance to effectively design the device during and around its operation; the friction model by affecting the friction coefficient depending on energy exchange to ensure a reasonable friction percentage; and the alternator force by affecting the alternator factor based on the change in stroke length to encourage stability. With these mechanisms in place, only steady state solutions are relevant. Later, after optimized device parameters have been established, the tuners are disabled to allow the study of controlled and uncontrolled transient behavior. For now, Table 1 describes the case that is considered for testing and validation.

Table 1: Parameters and steady state performance characteristics for the base case used for testing and validation.

\begin{tabular}{|r|l|}
\hline Bore & $64 \mathrm{~mm}$ \\
\hline Maximum stroke & $200 \mathrm{~mm}$ \\
\hline Port closing distance from cylinder head & $133 \mathrm{~mm}$ \\
\hline Equivalence ratio & 0.6 \\
\hline EGR & 0.12 \\
\hline Intake pressure & $1.05 \mathrm{bar}$ \\
\hline Intake temperature & $51.9^{\circ} \mathrm{C}$ \\
\hline Cylinder mixture mass & $0.484 \mathrm{~g}$ \\
\hline Wall temperature & $277^{\circ} \mathrm{C}$ \\
\hline Spring stiffness & $30 \mathrm{kN} / \mathrm{m}$ \\
\hline &
\end{tabular}




\begin{tabular}{|r|l|}
\hline Total moving mass & $2.39 \mathrm{~kg}$ \\
\hline Hohenberg leading parameter, $a$ & 65 \\
\hline Stroke length & $181 \mathrm{~mm}$ \\
\hline Compression ratio & 20.2 \\
\hline Frequency & $46.9 \mathrm{~Hz}$ \\
\hline Power & $22.7 \mathrm{~kW}$ \\
\hline System efficiency & $35.5 \%$ \\
\hline Mean alternator force & $1.27 \mathrm{kN}$ \\
\hline Peak cylinder pressure & $106 \mathrm{bar}$ \\
\hline Maximum translator acceleration & $16.3 \mathrm{~km} / \mathrm{s}^{2}$ \\
\hline Fuel energy added per cycle & $1.38 \mathrm{~kJ}$ \\
\hline
\end{tabular}

The next plots (Figure 36 - Figure 41) are used to describe this system in more detail. The first shows the velocity of the translator as a function of its position.

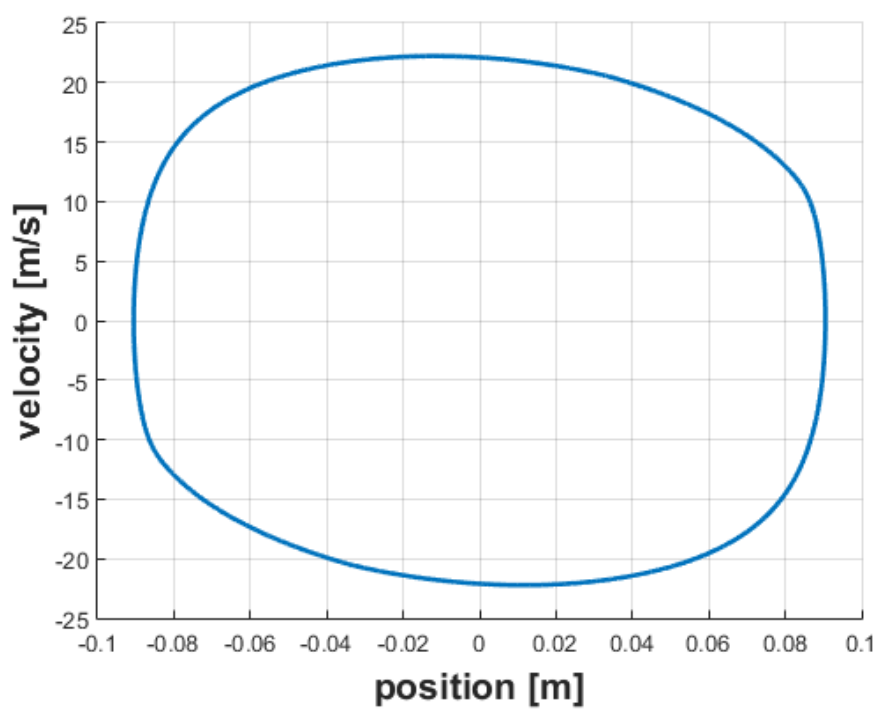

Figure 36: Velocity versus position profile for the testing and validation base case.

The position-velocity profile shown in Figure 36 displays attributes which are seen to be typical for the dual FPLE. The steep sides indicate high acceleration around top dead center and short residence time around the end of the stroke. The velocity profile is also skewed so that maximum velocity occurs prior to the midpoint position $(x=0)$. This effect is dependent on the relative dominance of the springs and is de-emphasized as the spring stiffness increases. The sharp increase in acceleration prior to top dead center is more evident in the velocity-acceleration profile shown in Figure 37. 


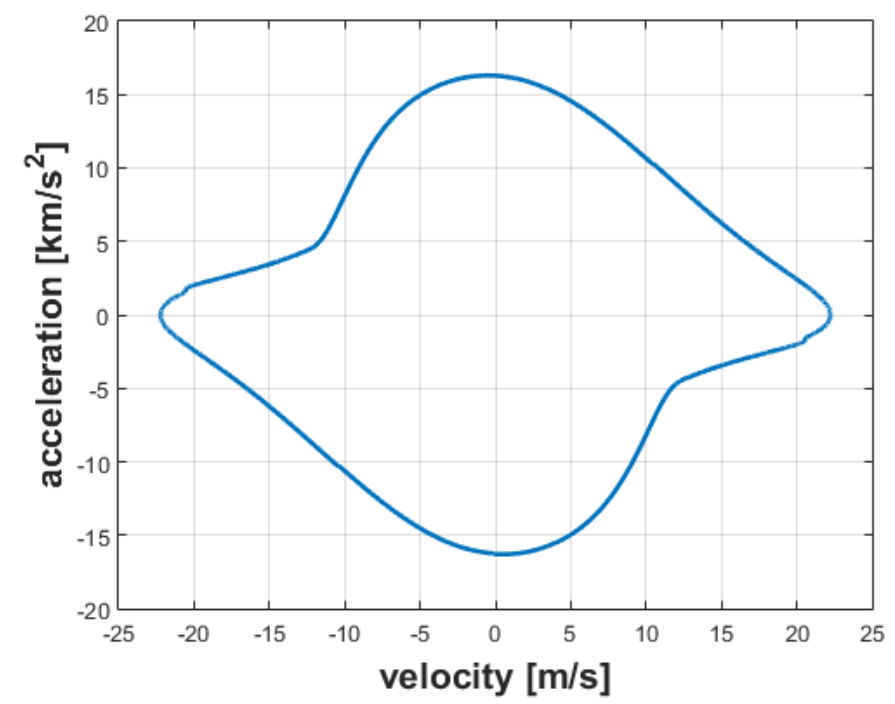

Figure 37: Acceleration versus velocity profile for the testing and validation base case.

Combustion heat release causes rapid increase in translator acceleration prior to top dead center position. Also, a small discontinuity can be seen just prior to the combustion event and is caused by the port opening and instantaneous gas exchange process occurring within the opposite cylinder. The pressure as a function of position is shown in Figure 38.

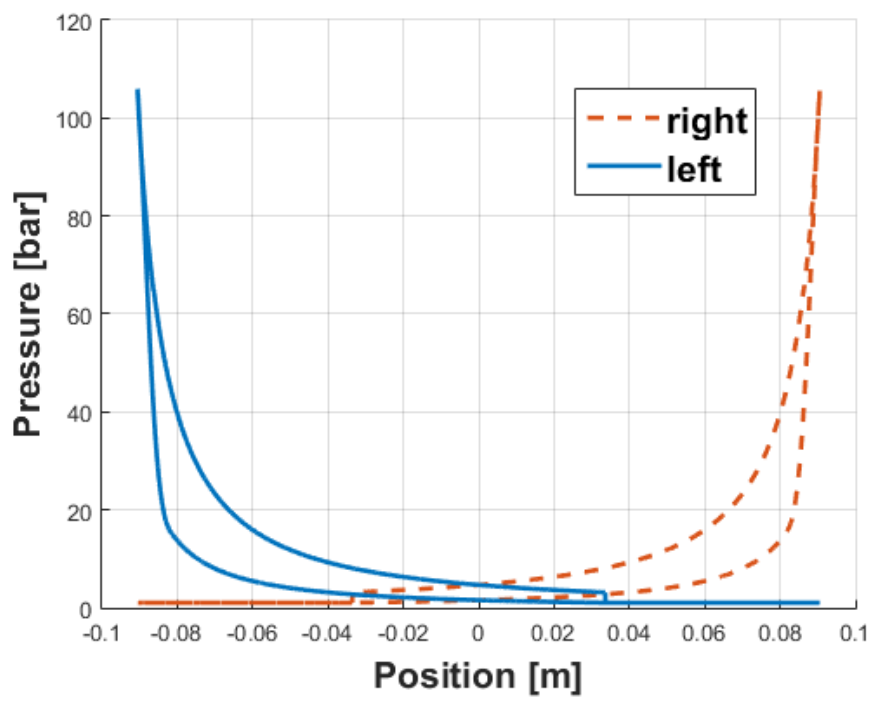

Figure 38: Pressure versus position profile for the testing and validation base case.

The pressure profiles in Figure 38 show the rapid heat addition from combustion as well as the instantaneous drop in cylinder pressure that corresponds to the simplified gas exchange model. Pressure and heat transfer to the cylinder walls are shown as functions of time in Figure 39. 

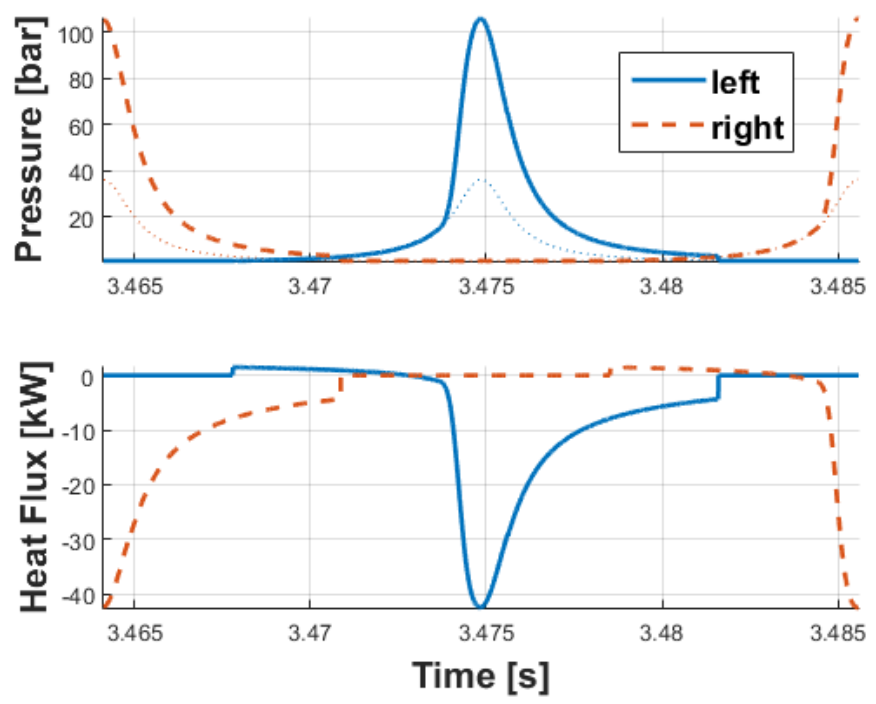

Figure 39: Pressure and heat flux as functions of time for the testing and validation base case.

In the pressure profiles, dotted lines represent the motoring pressure curves which separate from actual cylinder pressure at the point of ignition. Discontinuities in the heat flux profiles are due to the simple gas exchange model which does not include heat transfer. Heat flux rises sharply due to combustion and falls off more slowly than the corresponding pressure profile. The next figure demonstrates the oscillatory nature of power output and system efficiency over one cycle of system operation.
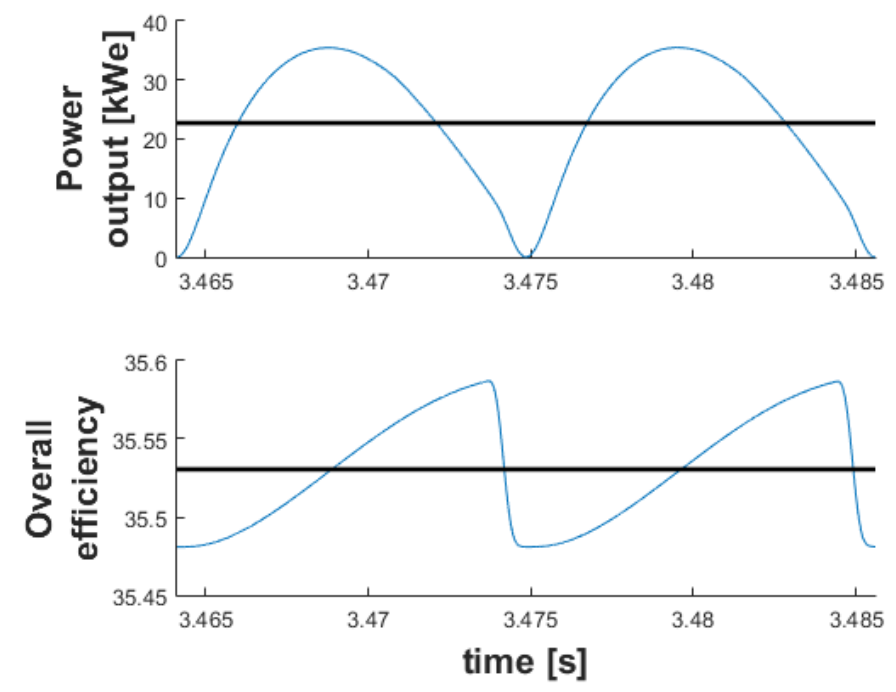

Figure 40: Power and efficiency as functions of time for the testing and validation base case.

Power is proportional to the velocity squared - the product of force and velocity where the force is defined as proportional to the velocity. Thus, the power output profile in Figure 40 is shown to oscillate at twice the cycle frequency of the translator. Efficiency is also shown to oscillate within a narrow band due to the periodic nature of combustion events and power output. The solid black lines correspond to 
the mean power and efficiency values over the cycle. Mean efficiency over a complete cycle is used to define the steady state system efficiency. The final figure given for the base case shows the energy loss distribution at steady state.

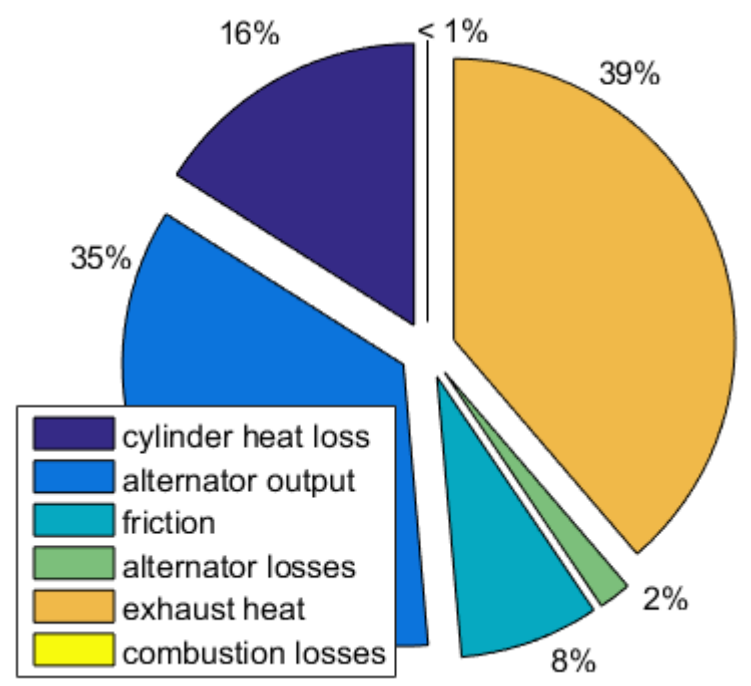

Figure 41: Energy balance for the testing and validation base case.

The pie chart in Figure 41 shows exhaust heat rejection as the leading cause for energy inefficiency. Combined with cylinder heat transfer, more than half $(55 \%)$ of the fuel energy is lost to heat. Along with the frictional losses, these values corroborate well with the energy consumption findings presented by DLR researchers in [41]. Additionally, high efficiency power output (relative to conventional engine technology) is shown for this un-optimized case, but it is worth noting that peripheral components (such as fuel and air compressors), along with power conversion inefficiencies, will likely reduce the overall efficiency.

\subsubsection{Effects of Solution Method}

Within Simulink, a wide variety of solution method parameters can be specified. For the base case, a fixed step size of five microseconds is employed for a simulated time of 3.5 seconds to ensure a steady state solution is reached. While the fixed step approach is required due to the complexity of the model and the need to compile the model for rapid simulation deployment, various solvers are available ranging from first- to eighth-order accuracy. MathWorks ${ }^{\circledR}$ help documentation within Simulink provides guidelines for selecting an appropriate solver given model complexity, desired accuracy, and computational limitations. This sensitivity analysis demonstrates a process similar to that suggested by MathWorks ${ }^{\circledR}$ and begins with Table 2 which shows the importance of step size for the solution. 
Table 2: Effects of step size on the steady state solution while all other base case attributes are held constant.

\begin{tabular}{|c|c|c|c|c|c|c|}
\hline $\begin{array}{c}\text { Step } \\
\text { size [s] }\end{array}$ & $\begin{array}{c}\text { Time } \\
\text { required [s] }\end{array}$ & $\begin{array}{c}\text { File } \\
\text { size [MB] }\end{array}$ & $\begin{array}{c}\text { Peak } \\
\text { pressure [bar] }\end{array}$ & $\begin{array}{c}\text { Stroke } \\
\text { length [mm] }\end{array}$ & $\begin{array}{c}\text { Efficiency } \\
\text { [\%] }\end{array}$ & $\begin{array}{c}\text { Power } \\
\text { [kWe] }\end{array}$ \\
\hline $1.0 \mathrm{E}-4$ & 0.589 & 0.263 & unstable & unstable & unstable & unstable \\
\hline $5.0 \mathrm{E}-5$ & 0.829 & 12.14 & 179.7 & 187.8 & 53.30 & 33.47 \\
\hline $1.0 \mathrm{E}-5$ & 2.22 & 59.64 & 108.2 & 181.6 & 36.90 & 23.75 \\
\hline $0.5 \mathrm{E}-5$ & 4.19 & 117.6 & 105.9 & 181.1 & 35.53 & 22.71 \\
\hline $1.0 \mathrm{E}-6$ & 29.1 & 562.7 & 103.6 & 180.7 & 34.50 & 22.04 \\
\hline $0.5 \mathrm{E}-6$ & 68.4 & 1084 & 103.3 & 180.7 & 34.38 & 21.99 \\
\hline
\end{tabular}

As might be expected, computational time and file size increase linearly with reduced step size. However, improvement to model precision plateaus as step size continues to decrease. Modeling work presented by Newcastle and Beijing researchers utilized a step size of ten microseconds [35]. Given the desire to balance model precision, computation time, and file size, the base case step size appears to be reasonable. Next, Table 3 demonstrates how the order of accuracy of the Simulink solver can affect model precision.

Table 3: Effects of Simulink solver order of accuracy on the steady state solution while all other base case attributes held constant.

\begin{tabular}{|c|c|c|c|c|c|}
\hline $\begin{array}{c}\text { Simulink } \\
\text { solver, accuracy }\end{array}$ & $\begin{array}{c}\text { Time } \\
\text { required [s] }\end{array}$ & $\begin{array}{c}\text { Peak } \\
\text { pressure [bar] }\end{array}$ & $\begin{array}{c}\text { Stroke } \\
\text { length [mm] }\end{array}$ & $\begin{array}{c}\text { Efficiency } \\
\text { [\%] }\end{array}$ & $\begin{array}{c}\text { Power } \\
\text { [kWe] }\end{array}$ \\
\hline ode1 (Euler) & 3.11 & 106.9 & 181.2 & 35.94 & 22.90 \\
\hline ode2 (Heun) & 3.12 & 105.9 & 181.1 & 35.53 & 22.71 \\
\hline ode3 (Bogacki-Shampine) & 3.63 & 105.9 & 181.1 & 35.53 & 22.71 \\
\hline ode4 (Runga-Kutta) & 4.19 & 105.9 & 181.1 & 35.53 & 22.71 \\
\hline ode5 (Dormand-Prince) & 5.30 & 105.9 & 181.1 & 35.53 & 22.71 \\
\hline ode8 (Dormand-Prince) & 9.66 & 105.9 & 181.7 & 35.53 & 22.71 \\
\hline
\end{tabular}

Simulink offers the selection of various fixed step solvers, each with different orders of accuracy. In Table 3, six of the solvers are examined, each with the order of accuracy indicated by the value tacked to the abbreviation 'ode.' For example, ode1 refers to an ordinary differential equation solver of first order accuracy. As the order or accuracy increases, the computation time required increases. However, model precision ceases to be improved after the increase from first to second order accuracy. For the demonstrated base case, the fourth order solver was selected to ensure precision. To reduce the simulation time while remaining conservative against the possibility that different simulations may require higher solver accuracy, the third order solver is selected.

Finally, the virtual time at which the simulation ends (termed 'Stop Time' in Simulink) can be varied to allow more or less time for the simulated system to achieve steady state operation. This is not to be confused with the computational time required to complete the simulation that has been discussed so far. As an example, Figure 42 demonstrates the base case simulation achieving steady state operation after 
around 0.2 seconds. The simulation stop time was set for one second, and the time required to complete the simulation (computational time) was 1.24 seconds.
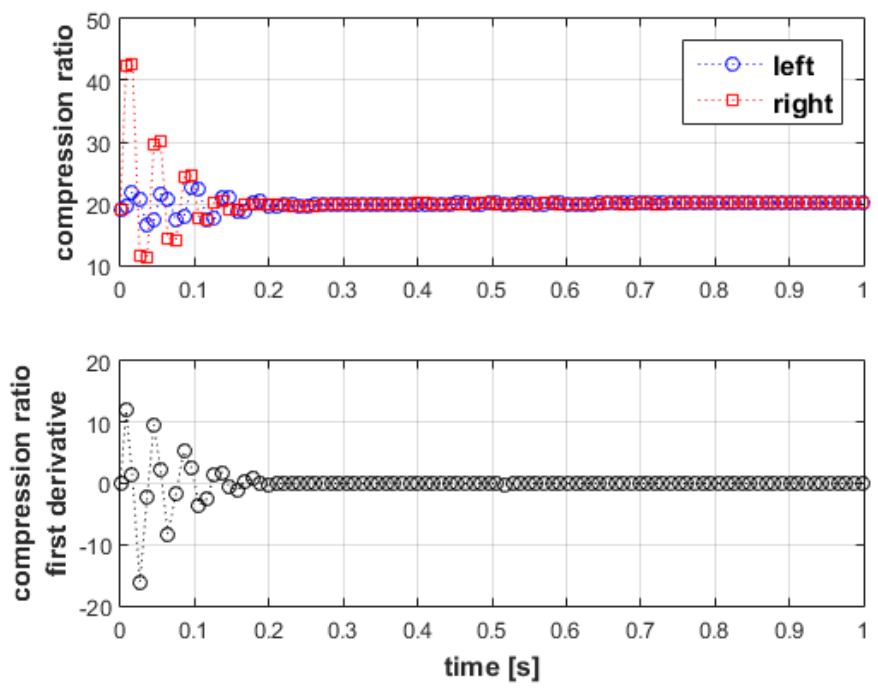

Figure 42: Example of the testing and validation base case achieving steady state after 1 second of virtual time. The results of this analysis are presented in Table 4.

Table 4: Effects of stop time on steady state simulation results.

\begin{tabular}{|c|c|c|c|c|c|c|}
\hline $\begin{array}{c}\text { Stop } \\
\text { time [s] }\end{array}$ & $\begin{array}{c}\text { Time } \\
\text { required [s] }\end{array}$ & $\begin{array}{c}\text { File } \\
\text { size [MB] }\end{array}$ & $\begin{array}{c}\text { Peak } \\
\text { pressure [bar] }\end{array}$ & $\begin{array}{c}\text { Stroke } \\
\text { length [mm] }\end{array}$ & $\begin{array}{c}\text { Efficiency } \\
\text { [\%] }\end{array}$ & $\begin{array}{c}\text { Power } \\
\text { [kWe] }\end{array}$ \\
\hline 1.0 & 1.24 & 34.42 & 105.7 & 181.0 & 35.51 & 22.61 \\
\hline 1.5 & 1.67 & 51.10 & 105.8 & 181.0 & 35.50 & 22.69 \\
\hline 2.0 & 2.23 & 67.76 & 105.4 & 181.0 & 35.51 & 22.92 \\
\hline 2.5 & 2.64 & 84.40 & 105.8 & 181.1 & 35.52 & 22.81 \\
\hline 3.0 & 3.21 & 101.1 & 106.1 & 181.1 & 35.53 & 22.71 \\
\hline 3.5 & 3.64 & 117.7 & 105.9 & 181.1 & 35.53 & 22.71 \\
\hline
\end{tabular}

Table 4 shows no obvious trend as the virtual stop time is increased aside from the computational time required to complete the simulation and resulting file size required to store the results. While the demonstrated base case was presented with a stop time at 3.5 seconds to ensure steady state operation, the stop time can be reduced to shorten computation time and file size as necessary. It is noted that different parameter cases will result in a range of dynamic response times, so a conservative stop time of two seconds is chosen as the lower limit.

\subsubsection{Effects of Heat Transfer Model}

The convection heat transfer model previously described depends on the gradient between gas and wall temperature, instantaneous cylinder surface area, and an instantaneous heat transfer coefficient. While the correlation by Hohenberg was presented for use in this model, various empirically correlated formulae, each with strengths and weaknesses, can be found in the literature. Unfortunately, 
these empirical relationships are based upon slider-crank motion rather than the unusual and variable motion of the linear engine system. Piston velocity is the usual reference for the in-cylinder charge motion that enhances the heat transfer, along with instantaneous gas temperature, pressure and cylinder volume. Hohenberg's formulation is chosen because of its relative simplicity and recent application by Newcastle and Beijing researchers [35]. However, this section compares the model by Hohenberg with models by Woschni [99], Annand [100], and Chang [86]. Note that the model by Chang is also referred to as the $\mathrm{HCCl}$ modified Woschni correlation. These four models are summarized well in [101], and Hohenberg's relationship is provided in Eq. 51 (repeated here for reference).

$$
h=a * V^{b} * P^{c} * T^{d} *(\bar{v}+1.4)^{e}
$$

Eq. 51

The Hohenberg correlation is examined with respect to its empirically correlated parameters. Referring back to Eq. 51, five parameters are used to define the correlation: $a$ is the leading coefficient equal to 130 in the original publication but set to 65 in the base case to scale down the heat transfer; $b$ is the volume exponent equal to $-0.06 ; c$ is the pressure exponent equal to $0.8 ; d$ is the temperature exponent equal to -0.4 ; and $e$ is the velocity exponent equal to 0.8 . Figure 43 shows the results of the Hohenberg heat transfer model parametric sweeps with the Hohenberg parameters normalized against the base case values so that the base case corresponds to unity in each of the figures. The figures demonstrate that each of the correlated parameters can have significant impact on translator dynamics and can play an important role in heat loss and subsequent steady state performance.
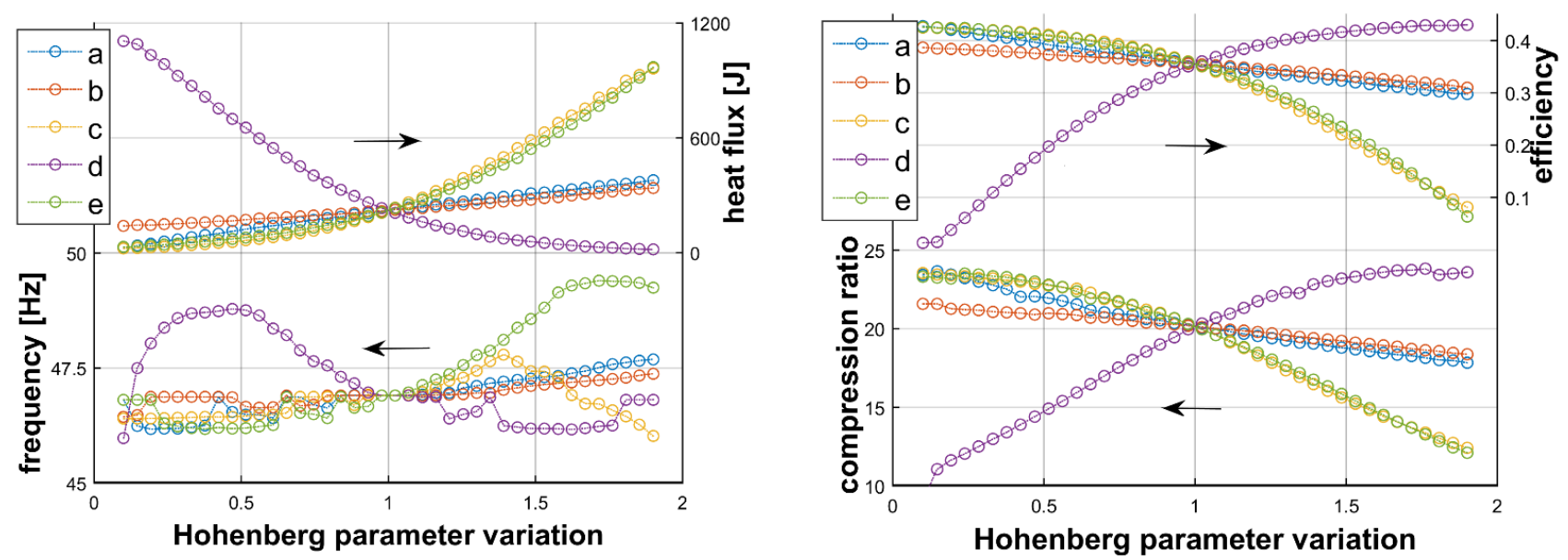

Figure 43: Effects of Hohenberg parameters on frequency and heat flux.

Figure 44 and Figure 45 show the effect of using four different formulae to describe the heat loss. In these four cases, the total heat lost is equal to the base case (16\%), but the distribution of the heat loss with respect to the translator position is shown to yield subtle difference in stroke length and peak pressure. The differences associated with the heat transfer models is most noticeable in terms of 
instantaneous heat flux, whereas the thermodynamic relationships tend to mask the differences in the pressure domain. This is explained by the fact that the total heat loss over the time span is most important, rather than the precise heat loss at any moment. In addition, the formulae do not differ substantially and all employ the temperature difference in calculating the heat flux.
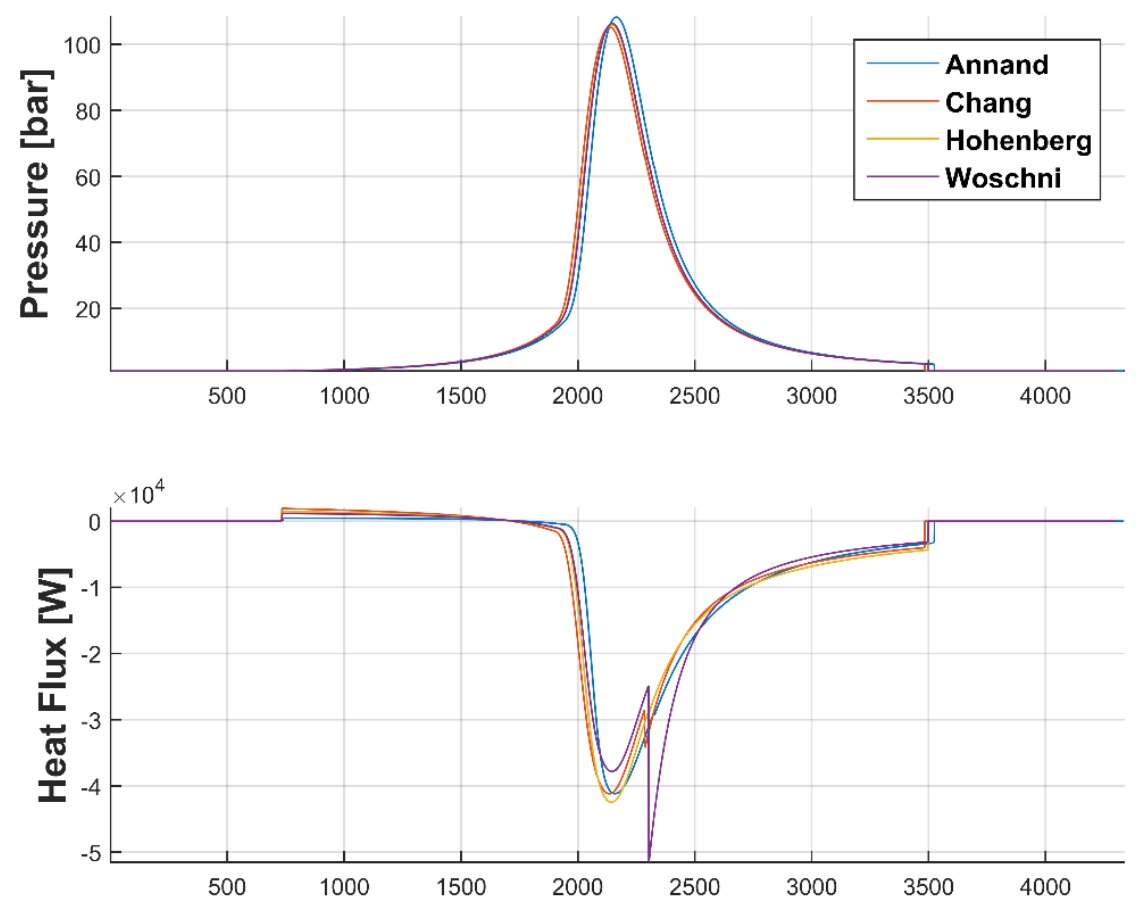

Figure 44: Comparison of cylinder pressure and heat flux profiles four heat transfer models.

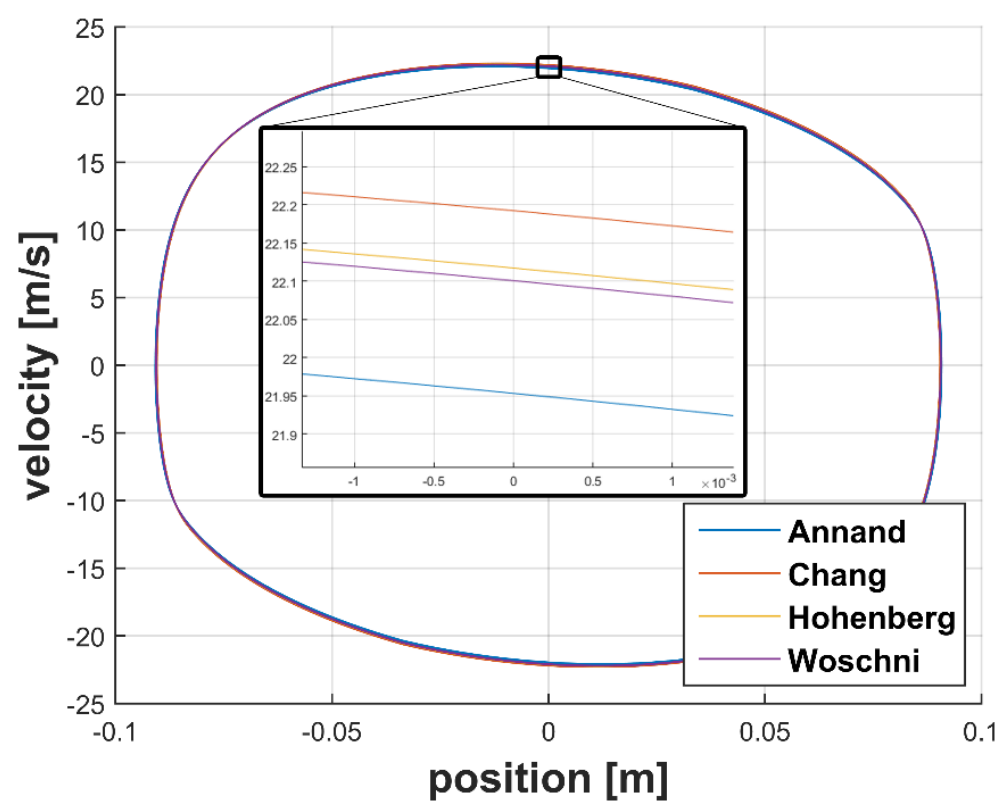

Figure 45: Comparison of translator dynamic profiles four heat transfer models. 


\subsubsection{Effects of Ignition Timing Model}

The combustion process is perhaps the most challenging process to represent accurately with the 1-D pressure model. Using the knock integral method as described for this numeric model enables prediction of the ignition timing, but it will be shown in this section that the empirical relationship can have significant effect on the start of combustion and subsequent device operation. The base case employs the relationship given in Eq. 55 which was correlated to CFR engine data. This relationship is repeated here for reference.

$$
\tau=A \omega \phi^{-x} \exp \left(\frac{b}{T} P^{n}\right)
$$

Eq. 55

The given formulation employs four parameters: the leading coefficient, $A=9.291 \cdot 10^{-7}$; the equivalence ratio exponent, $x=16909$; the temperature coefficient, $b=-0.1121$; and the pressure exponent, $n=$ -0.688 . Starting with the leading coefficient $(A)$, Figure 46 shows how reducing $A$ can advance the combustion timing and lead to adverse work around top dead center.

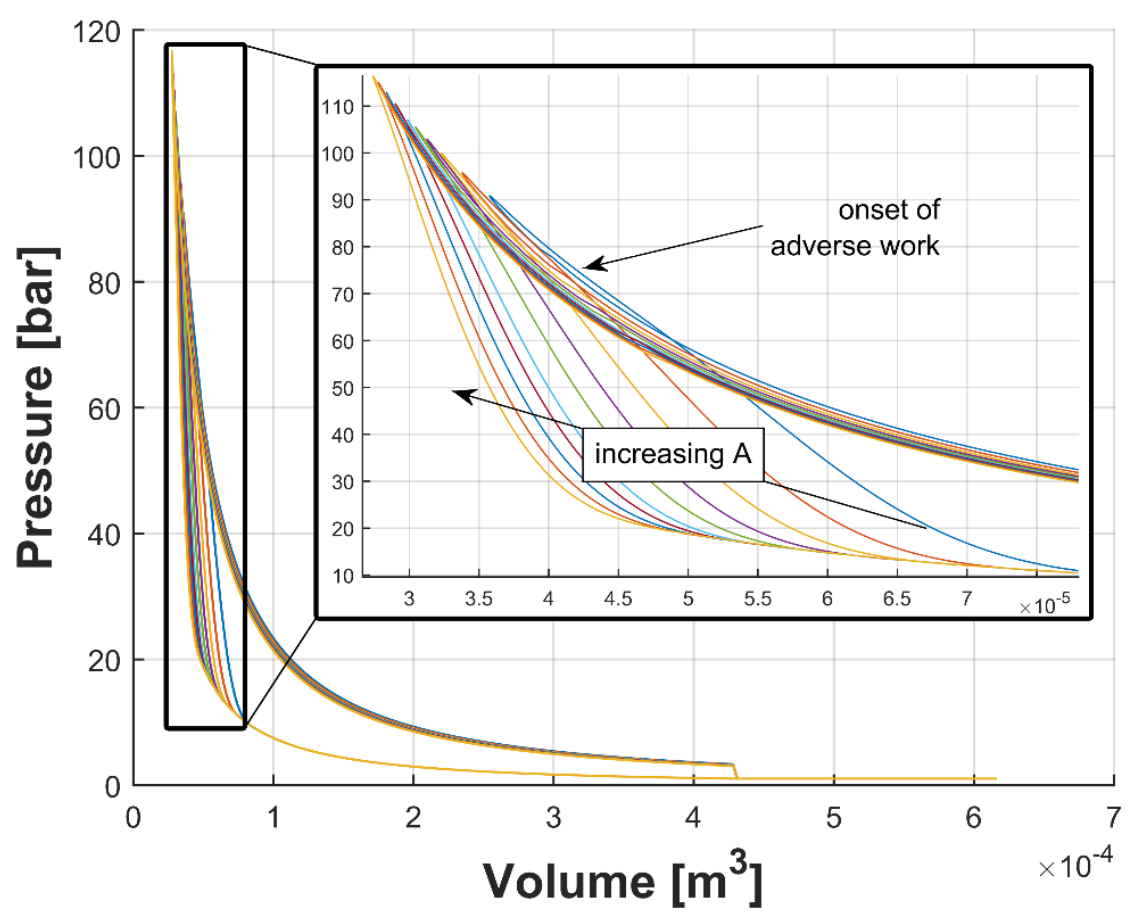

Figure 46: Effect of varying the CFR ignition model leading coefficient $(A)$ on pressure profile.

The departure of the pressure curves from the motoring curve in pressure-volume diagram corresponds to the earlier or later ignition due to varying values of $A$. The largest value of $A$ implies the longest ignition delay in the Eq. 54 integral, and results in pressure rise later in the cycle. In contrast, the very early onset of combustion causes rapid pressure rise as the piston is still moving towards the head, and results in adverse pressure and negative work. This negative work arises because heat is being lost 
to the cylinder walls at a higher rate than combustion is contributing to pressure rise in this location. Just as in a slider-crank engine, both early and late ignition can be detrimental to the efficiency. If combustion were to occur all on the compression stroke, then without heat transfer, the rising and falling parts of the pressure curve would lie on top of one another around top dead center. With heat transfer, unacceptable losses occur. Performing a similar parameter sweep of the temperature coefficient $(b)$ provides insight on the effect of ignition timing on system stability. Figure 47 shows the position-velocity profiles for a range of cases where $b$ is varied.

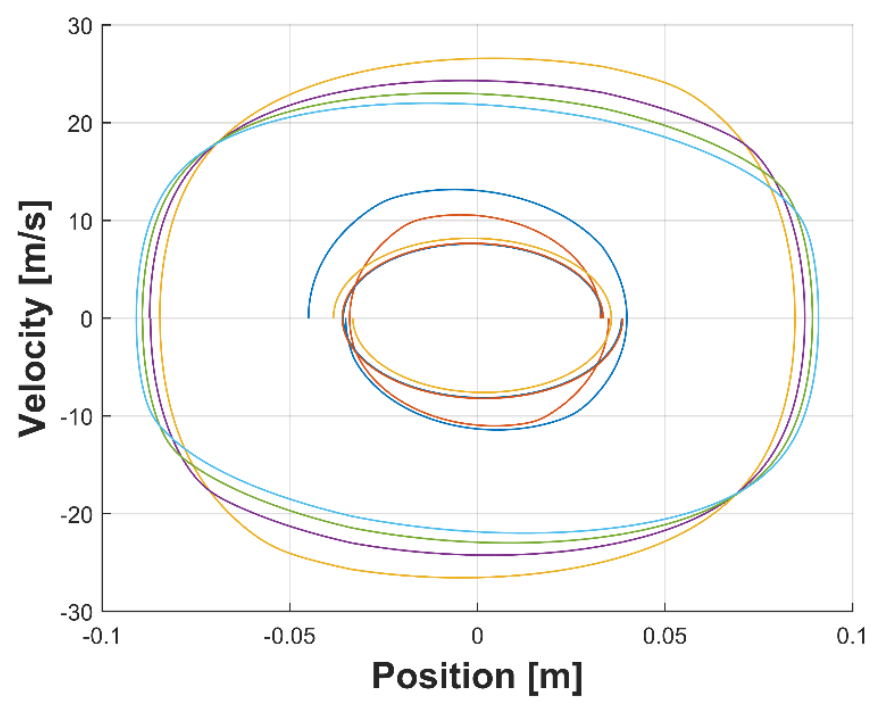

Figure 47: Effect of varying the CFR ignition model temperature coefficient $(b)$ on translator dynamic profiles. The color of each profile corresponds to the pressure profiles in Figure 48.

The position-velocity profiles with the smallest stroke shows the lack of cyclic repeatability: the SALEG is in the process of "ringing down" and will eventually stall. The cause becomes clear with the pressure-volume plots shown in Figure 48. 


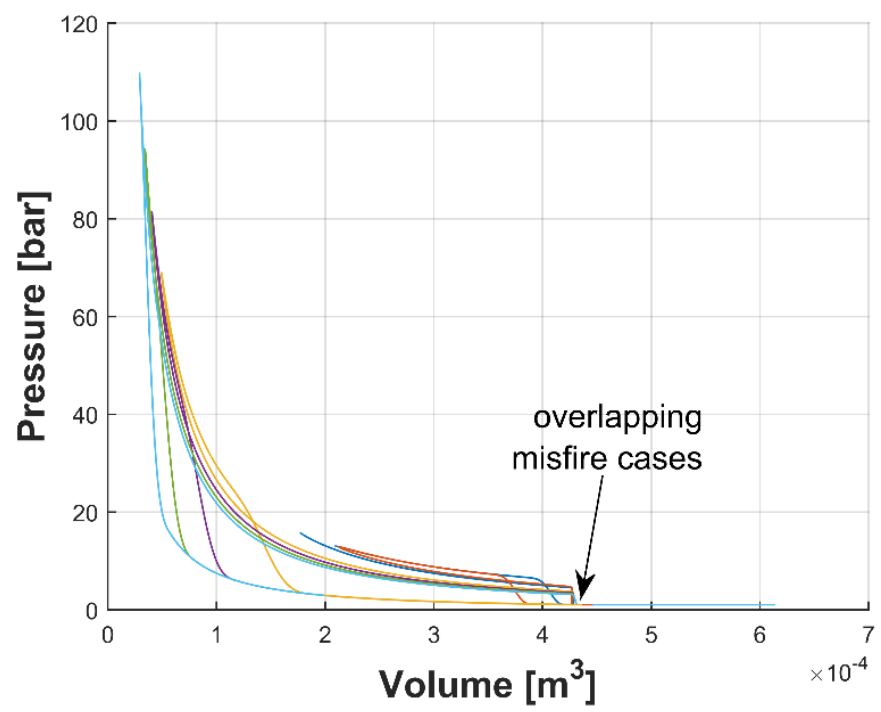

Figure 48: Effect of varying the CFR ignition model temperature coefficient $(b)$ on pressure profiles. The color of each profile corresponds to the dynamic profiles in Figure 47.

When the ignition is sufficiently late, the peak pressures lie above 80 bar and the SALEG enjoys a full stroke; but for the earliest ignition conditions, stable operation cannot be achieved due to the excessive adverse work and eventual misfire. Figure 49 examines the case where power density (due to varying equivalence ratio) is varied rather than the point of ignition.

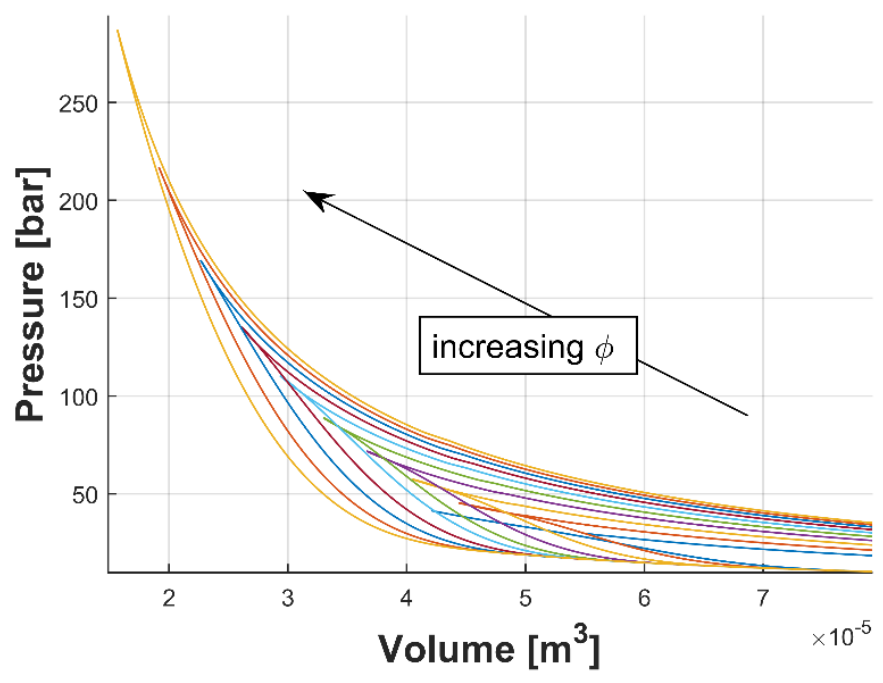

Figure 49: Effect of equivalence ratio on CFR model ignition timing prediction on cylinder pressure.

In these pressure curves, negligible adverse work is evident because ignition does not occur early enough to allow heat transfer to compete with the combustion pressure rise. The ignition timing at larger volumes when less fuel is added coincides with the reduced stroke. Peak pressure is reduced not only due to the reduced amount of heat that is released during combustion, but because the stroke (and compression ratio) is reduced. The effects of $A, b$, and equivalence ratio are summarized along with the remaining two parameters in Figure 50. 

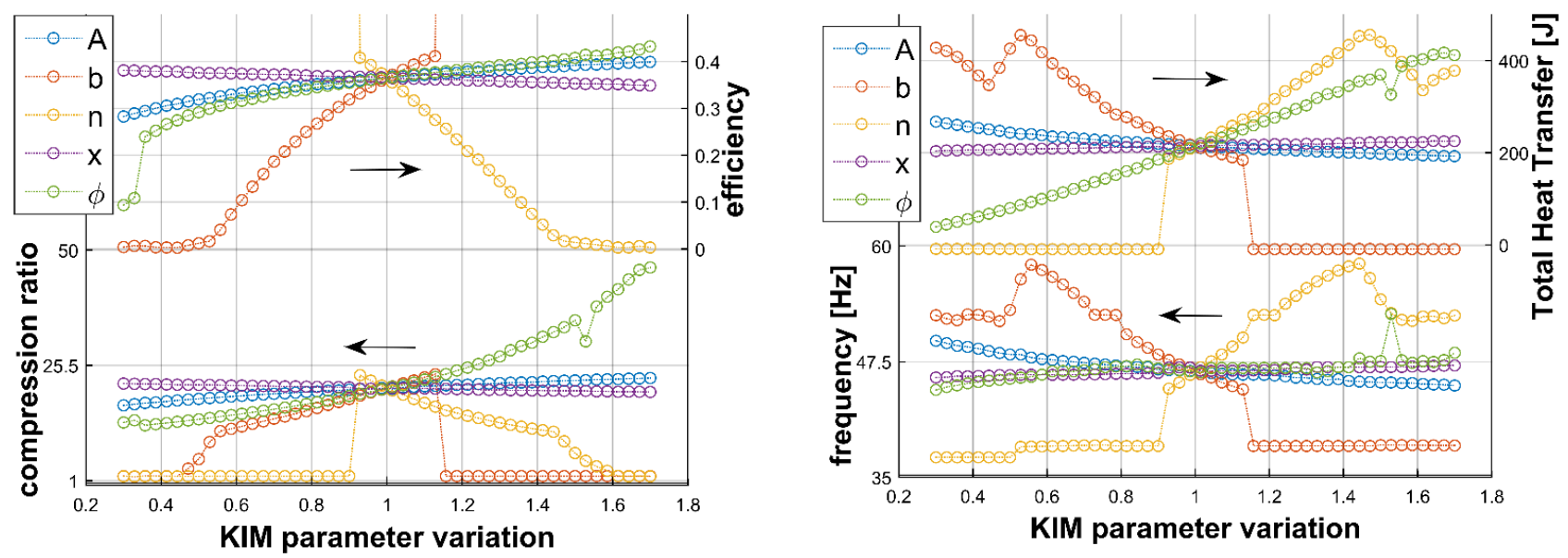

Figure 50: Effects of varying CFR model ignition timing parameters on compression ratio, efficiency, frequency, and total heat transfer.

The previous two plots show how the ignition timing can have significant impact on system performance and that ignition model parameters play a very important role. For both plots, the horizontal axis corresponds to the parameter being varied normalized against the base case so that the base case is seen at unity. Parameters $b$ and $n$ are seen to have the greatest impact on ignition timing as indicated by the unstable operation points (points corresponding to efficiency of zero or infinity).

$$
\tau=1.3 \cdot 10^{-7} \cdot P^{-1.05} \cdot \phi_{F O}^{-0.77} \cdot \chi_{O_{2}}^{-1.41} \cdot \exp \left(\frac{E}{R \cdot T}\right)
$$

The alternative model given in Eq. 56 and recently used in free piston modeling work by Lee [78] involves a slightly different structure and takes into account EGR effects on ignition timing. The model parameters are correlated to combustion data gathered from a rapid compression machine operating with iso-octane. Figure 51 shows the comparison between the base case with the CFR model and RCM ignition models. 


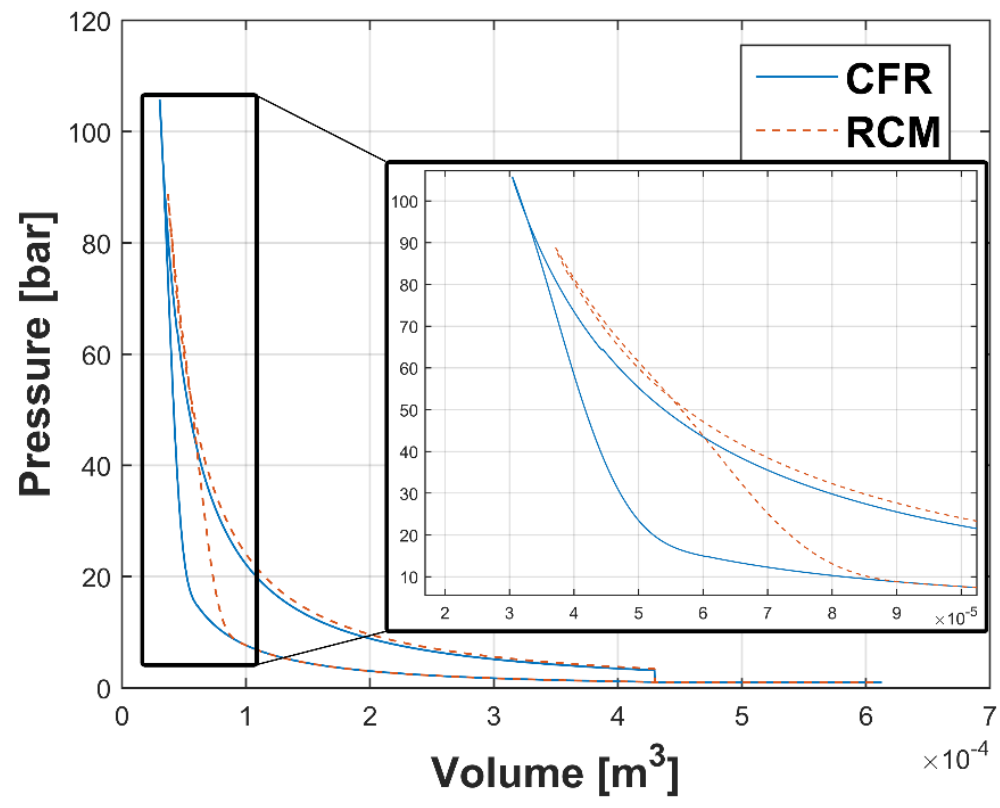

Figure 51: Comparison between CFR and RCM ignition timing models on cylinder pressure.

The base case with the RCM ignition model shows significantly earlier combustion timing as compared to the CFR model. As before, the advanced ignition timing causes earlier rise in pressure leading to negative work around TDC, shorter stroke length, smaller compression ratio, and reduced peak pressure. Examining the effects of EGR within the RCM model more closely, Figure 52 shows that EGR has little effect on performance, but increased EGR delays ignition timing while at the same time reducing peak pressure because of reduced power density.

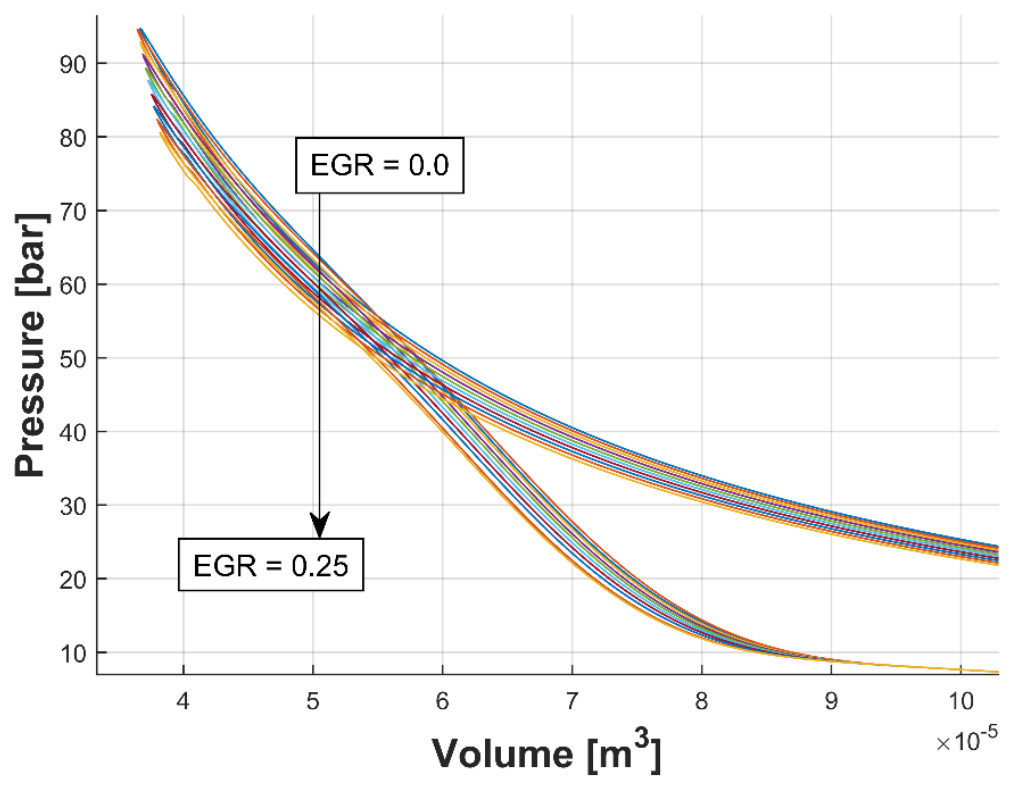

Figure 52: Effects of EGR on the base case cylinder pressure with the RCM ignition model. 
Because the combustion timing is of paramount importance for accurate modeling of the engine, the simplifications made to represent the engine with the 1-D pressure model add significant uncertainty to the model. The stochastic nature of gas exchange, heat transfer, and combustion are exacerbated within the free piston whose dynamic response is greatly interdependent on many variables. The choice to employ the CFR engine within the base case is no more or less supported than the RCM model, and it is emphasized that additional research is necessary for the free piston engine operating within an autoignition combustion regime.

\subsubsection{Effects of Combustion Heat Release Profile}

The single Wiebe heat release profile is presented in Eq. 53 and its corresponding mass fraction burn profile given in Eq. 52. For reference, these equations are repeated below. While the Wiebe function is classically used in 1-D pressure modeling of an engine, alternatives have been presented throughout the literature. Additionally, combustion is a highly stochastic process dependent on cylinder wall temperature, cylinder geometry, mixture stratification, fuel properties, and rate of pressure rise. For the slider-crank engine, single zone modeling at a constant engine speed allows correlations to be drawn for $\mathrm{HCCl}$ combustion duration, efficiency, and heat release profile based on engine operation parameters and known crank angle [92]. However, correlations for the free piston engine are more difficult to form and depend on experimental data which is more difficult to gather. Nonetheless, examining the effects of combustion duration and burn profile on translator dynamics is important to understand the impact of assumptions made within the numeric model. Thus, Figure 53 and Figure 54 show the results of varying the combustion duration through a range of values from 0.1 milliseconds to 0.03 seconds. Note that because the shortest duration is near the same order of magnitude as the base case solution step size, the step size was reduced to one microsecond for this set of simulations.

$$
\begin{gathered}
\chi(t)=1-\exp \left[-a\left(\frac{t-t_{S O C}}{C_{d}}\right)^{1+b}\right] \\
\frac{d Q_{H R}}{d t}=\eta_{\text {comb }} E_{\text {fuel }}\left[a \frac{b+1}{C_{d}}\left(\frac{t-t_{S O C}}{C_{d}}\right)^{b} \exp \left(-a\left(\frac{t-t_{S O C}}{C_{d}}\right)^{1+b}\right)\right]
\end{gathered}
$$




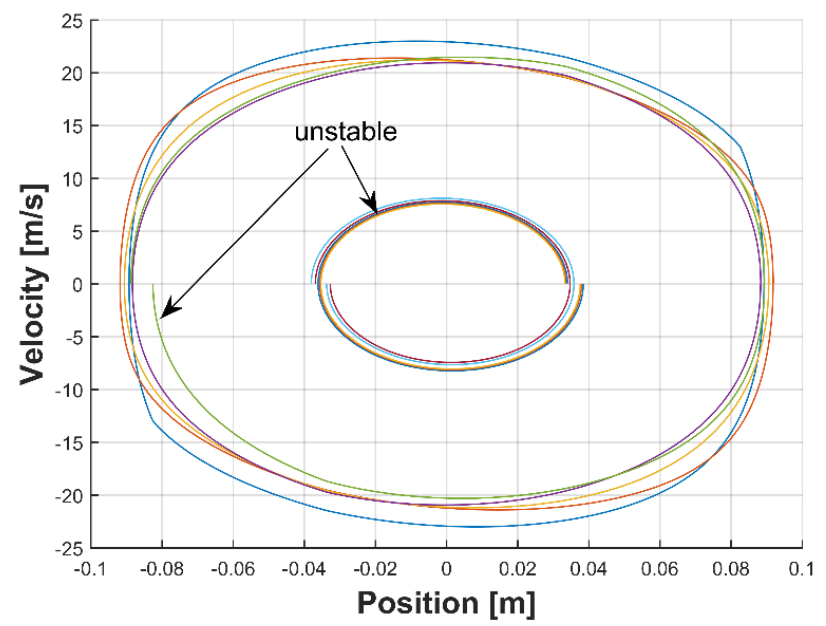

Figure 53: Effect of varied combustion duration on translator dynamic profiles. The color of each profile corresponds to those seen in Figure 54 .

The dynamics of the translator are demonstrated in Figure 53 for the given range of combustion duration values so that each profile represents a separate simulation wherein the combustion duration is assumed constant. For some cases, the combustion event is extended for too great a length of time leading to unstable behavior.

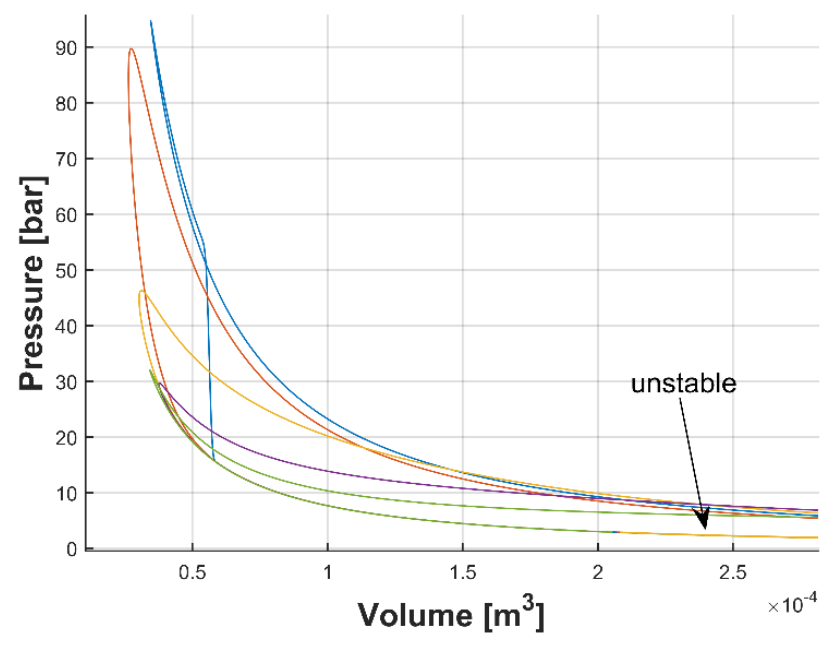

Figure 54: Effect of varied combustion duration on cylinder pressure. The color of each profile corresponds to those seen in Figure 53.

Trends for the variation of combustion duration are given in Figure 55. 

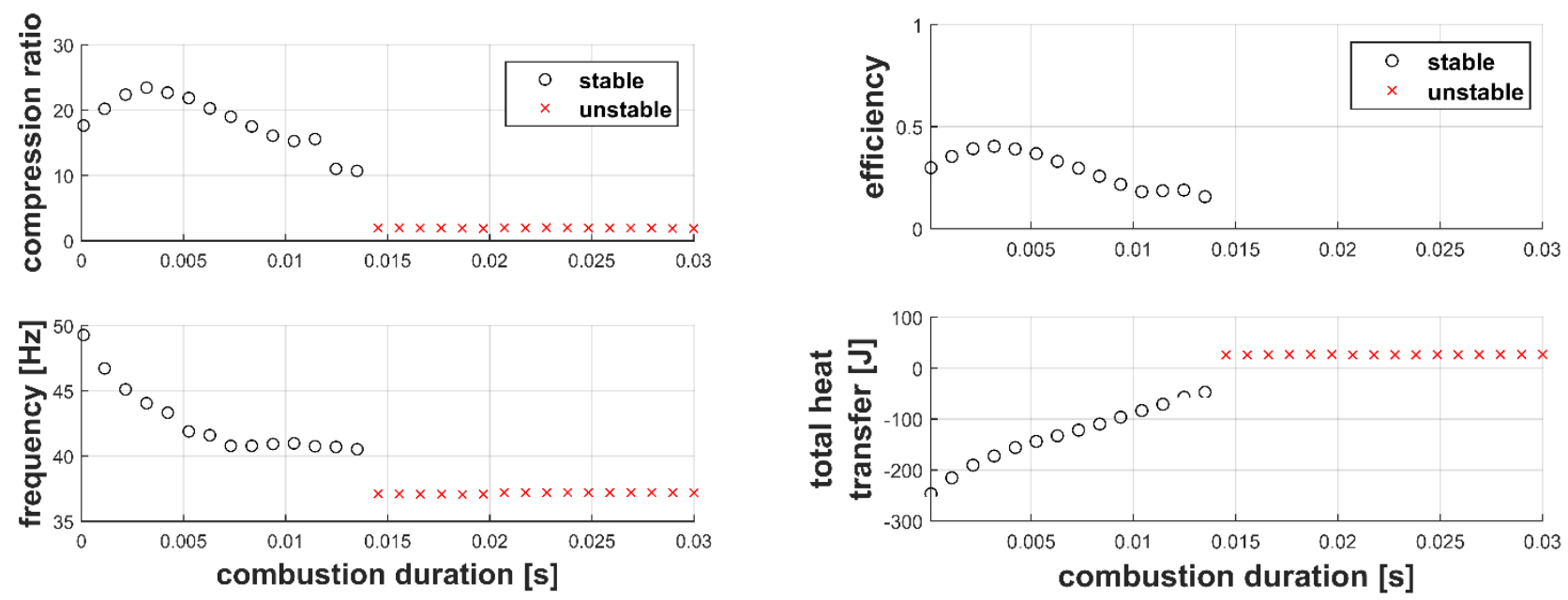

Figure 55: Effects of varied combustion duration on base case frequency, compression ratio, efficiency, and heat transfer. Stable and unstable cases are distinguished by ' 0 ' or ' $x$,' respectively.

The points marked with an ' $x$ ' indicate unstable systems, while the 'o' points can be used to identify trends for stable, steady state operation. Increasing combustion duration results in significant decrease to engine speed and reduced heat transfer losses. Relatively smaller changes are seen for compression ratio and efficiency.

Next, a second combustion heat release profile is considered within the model. The double Wiebe function is presented by Yasar et al. as a means to account for the cooler gas regions near the cylinder walls that burn more slowly [102]. In his dissertation, Shoukry applied single zone modeling with a variation of the double Wiebe profile to study a direct injection compression ignition free piston engine [103]. This formulation is given in Eq. 58.

$$
\frac{d Q_{i n}}{d t}=a \frac{Q_{i n, p}}{t_{p}}\left(M_{p}+1\right)\left(\frac{t}{t_{p}}\right)^{M_{P}} \exp \left[-a\left(\frac{t}{t_{p}}\right)^{M_{p}+1}\right]+a \frac{Q_{i n, d}}{t_{d}}\left(M_{d}+1\right)\left(\frac{t}{t_{d}}\right)^{M_{d}} \exp \left[-a\left(\frac{t}{t_{d}}\right)^{M_{d}+1}\right]
$$

The double Wiebe is composed of two Wiebe functions where the first captures the premixed burn phase and the second is for the diffusive burn phase. The shape factors $M_{p}$ and $M_{d}$ are given as 3 and 0.5 , respectively, and $a$ is taken to be 1.2. For a fixed amount of heat released during combustion, it is assumed that the heat released during premixed and diffusion burn phases must add up to the total heat (i.e. $Q_{i n}=Q_{i n, p}+Q_{i n, d}$ ). Similarly, the combustion duration must be the sum of the time spent in each of the burn phases $\left(C_{d}=t_{p}+t_{d}\right)$. Using the double Wiebe relationship, two profiles are formed for comparison and shown in Figure 56. It is noted that the author is not suggesting the use of the double Wiebe or any particular profile, but it has been shown to be a viable option depending on application so should be considered within the numeric model. 

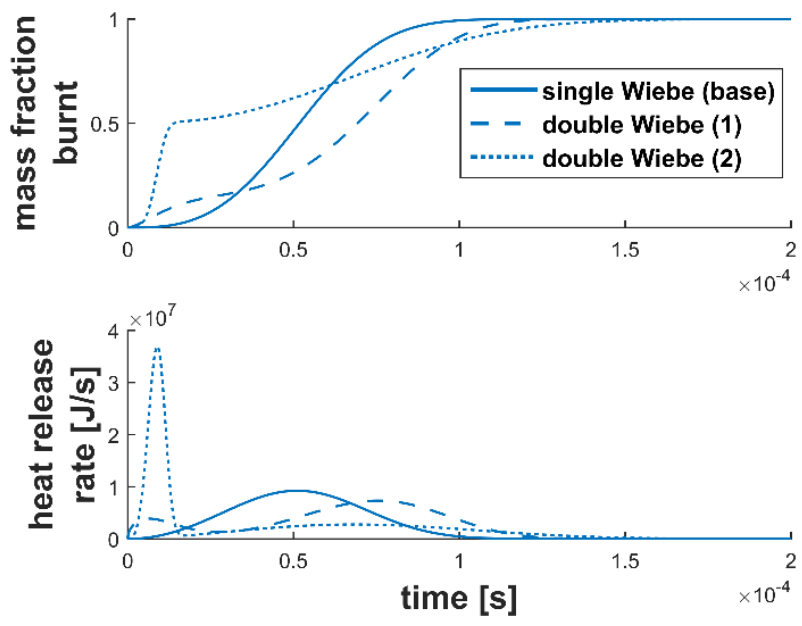

Figure 56: Comparison between single and double Wiebe heat release profiles.

Comparing the double Wiebe heat release profiles, the first shows a rapid initial burn of a small amount of fuel followed by a decrease in burn rate and second rapid burn phase until completion. The second shows burning of just over half of the fuel very early in the combustion process followed by a long steady diffusion burn phase. The effects of each burn profile are compared in Figure 57 and Table 5.
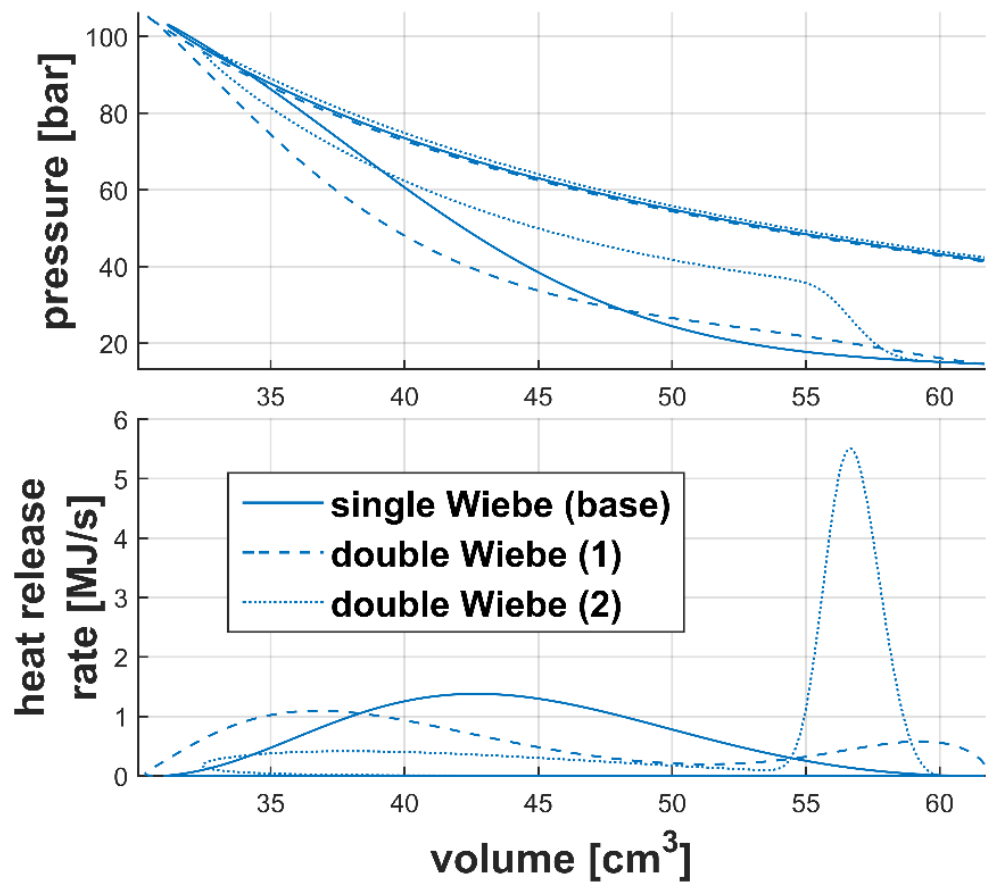

Figure 57: Effects of the varied burn profiles on cylinder pressure for the testing and validation base case. 
Table 5: Summary of effects for the base case simulation with varied burn profiles.

\begin{tabular}{|c|c|c|c|c|c|c|}
\hline Profile & $\begin{array}{c}\text { Peak } \\
\text { pressure } \\
\text { [bar] }\end{array}$ & $\begin{array}{c}\text { Stroke } \\
\text { length } \\
\text { [mm] }\end{array}$ & $\begin{array}{c}\text { Compression } \\
\text { ratio }\end{array}$ & $\begin{array}{c}\text { Efficiency } \\
\text { [\%] }\end{array}$ & $\begin{array}{c}\text { Power } \\
\text { [kWe] }\end{array}$ & $\begin{array}{c}\text { Frequency } \\
\text { [Hz] }\end{array}$ \\
\hline single Wiebe (base) & 103.2 & 180.7 & 19.69 & 34.49 & 22.25 & 47.02 \\
\hline double Wiebe (1) & 105.9 & 181.2 & 20.27 & 35.50 & 22.56 & 46.61 \\
\hline double Wiebe (2) & 96.94 & 179.9 & 18.86 & 32.75 & 21.39 & 47.83 \\
\hline
\end{tabular}

Examining the results of the varied combustion heat release profile, effects on performance are seen, but the high interdependence of the system makes assessment difficult. Based on Figure 56, the combustion duration is slightly longer for the double Wiebe profiles and longest for the second case because of the inclusion of a diffusion burn phase. The second double Wiebe profile also resulted in the poorest performance of the three simulations. This might be explained by the relatively long diffusion burn phase which allowed heat transfer losses to compete with combustion heat release to decrease the peak pressure and total energy in the system. While the frequency was not so greatly affected, stroke length and compression were decreased, contributing again to the lower efficiency and power output.

\subsubsection{Effects of Alternator Proportional Controller}

For the steady state simulation, a simple proportional controller is incorporated into the load equation to raise the value of the constant if stroke length increases and reduce the constant if stroke length falls. This approach is meant to encourage the stability of the translator for steady state analysis and does not represent any true effort in practical controller design for transient response. Furthermore, component design is integrated into the current simulation package so that transient analysis is irrelevant. The weighting factor $\left(W_{A}\right)$ is multiplied directly to the alternator load constant (as shown in Eq. 47), and its relationship with stroke (the proportional controller equation) is given in Eq. 59.

$$
W_{A}=1-G_{A}\left(\frac{S_{\text {targ }}-S}{L}\right)
$$

The weight applied to the alternator load depends on a gain $\left(G_{A}\right)$ and the difference between target stroke length $\left(S_{\text {targ }}\right)$ and instantaneous stroke length $(S)$ normalized by the system geometry $(L$ is the neutral clearance gap between piston head which is synonymous with half of the maximum stroke length). While the purpose of the controller is to encourage system stability, either the target stroke length or proportional gain can be varied to affect the dynamic response of the translator. For the base case, a low gain of 7.5 is used to allow the SALEG to find stability at different stroke lengths (and hence compression ratios) as other parameters are varied. To understand the effects of the gain value, two plots are given in Figure 58. 

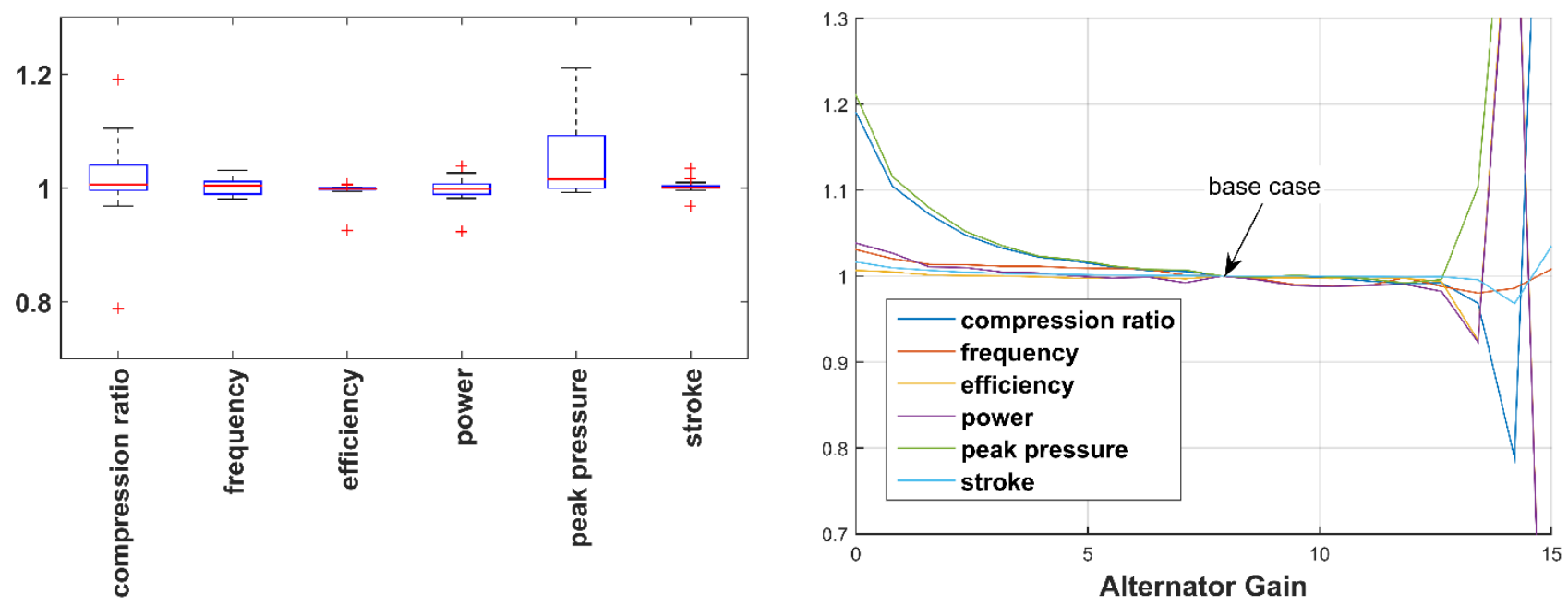

Figure 58: Normalized steady state performance characteristics compared when the alternator proportional controller gain is varied.

For the Figure 58 plots, each of the data are normalized against their own mean and then against the base case to present them together for comparison. The left-hand plot demonstrates the statistical spread of the data while the right-hand plot demonstrates that within a certain range, the gain has no significant effect on the performance of the SALEG. Performing the same type of parameter sweep for the target stroke length, the results are shown in Figure 59.

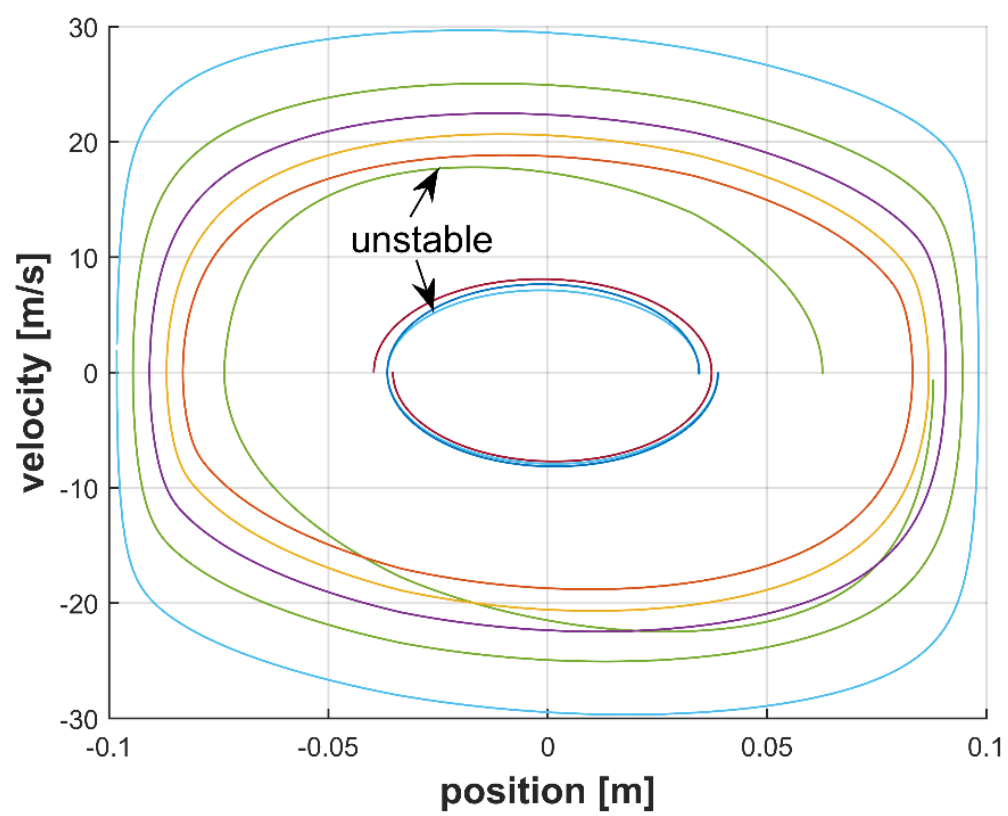

Figure 59: Effects of target stroke length on translator dynamics. Steady state stroke length and velocity are shown to increase as target stroke length is raised.

Demonstrated by the position-velocity plot is the significant effect of target stroke length on the steady state response of the SALEG. Except for the unstable cases, the magnitude of the positionvelocity profile grows with the value for target stroke length. By imposing a high stroke length target within 
the base case $(90 \%$ of the maximum possible stroke), the translator is encouraged to operate at a higher stroke (thus higher compression ratio). However, as demonstrated throughout the previous sensitivity analyses, the controller is not given the authority to affect the same steady state response for all sets of operating conditions.

\subsubsection{Comparison to Literature Results}

Experimental data for the SALEG is not available, so validation of the numeric model relies on the sensitivity studies given in the previous sections and comparison to experimental data that has been reported in the literature. Namely, data from two published experimental studies is presented here and compared with the numerical model. Details of the experimental models are presented in Table 6, and the available parameters are applied within the numeric model with the goal of achieving similar results between simulation and experiment.

Table 6: Summary of prototype device parameters validated in literature

\begin{tabular}{|c|c|c|}
\hline & $\begin{array}{c}\text { University of } \\
\text { Ulsan [83] }\end{array}$ & $\begin{array}{c}\text { West Virginia } \\
\text { University [17] }\end{array}$ \\
\hline Bore & $30 \mathrm{~mm}$ & $36.4 \mathrm{~mm}$ \\
\hline Maximum stroke & $31 \mathrm{~mm}$ & $50 \mathrm{~mm}$ \\
\hline Mass & $0.8 \mathrm{~kg}$ & $2.5 \mathrm{~kg}$ \\
\hline Pistons & 2 & 2 \\
\hline Fuel or LHV* & $46.296 \mathrm{MJ} / \mathrm{kg}$ & - \\
\hline Combustion duration & not given & $2.85 \mathrm{~ms}$ \\
\hline Intake pressure & $1.1 \mathrm{bar}$ & $1.35 \mathrm{bar}$ \\
\hline Intake temperature & $300 \mathrm{~K}$ & $341 \mathrm{~K}$ \\
\hline Equivalence ratio* & 1.0 & - \\
\hline Exhaust port location & $18 \mathrm{~mm}$ & $19 \mathrm{~mm}$ \\
\hline Spring stiffness & $2.9 \mathrm{~N} / \mathrm{mm}$ & 0 \\
\hline Ignition & $\mathrm{Spark}$ & Spark \\
\hline
\end{tabular}

*WVU researchers assumed $25 \mathrm{~J}$ of heat addition per stroke

Starting with the Ulsan model comparison, Figure 60 demonstrates the corroboration between the numeric model and Ulsan's prototype operation. The main difference between the two models is overprediction of peak cylinder pressure and translator velocity by the numeric model. With more intimate knowledge of the combustion and heat transfer characteristics, it would likely be possible to achieve a better fit. As it is, energy loss pathways and model parameters were estimated, while holding to the given physical parameters listed in Table 6, to achieve the given results. Additionally, Ulsan's prototype used spark to initiate the combustion event at a known translator location for steady state operation. The 
numeric model with RCM ignition timing model, EGR rate of 30\%, single Wiebe heat release profile, and combustion duration of three milliseconds is employed to approximate the combustion timing and event.
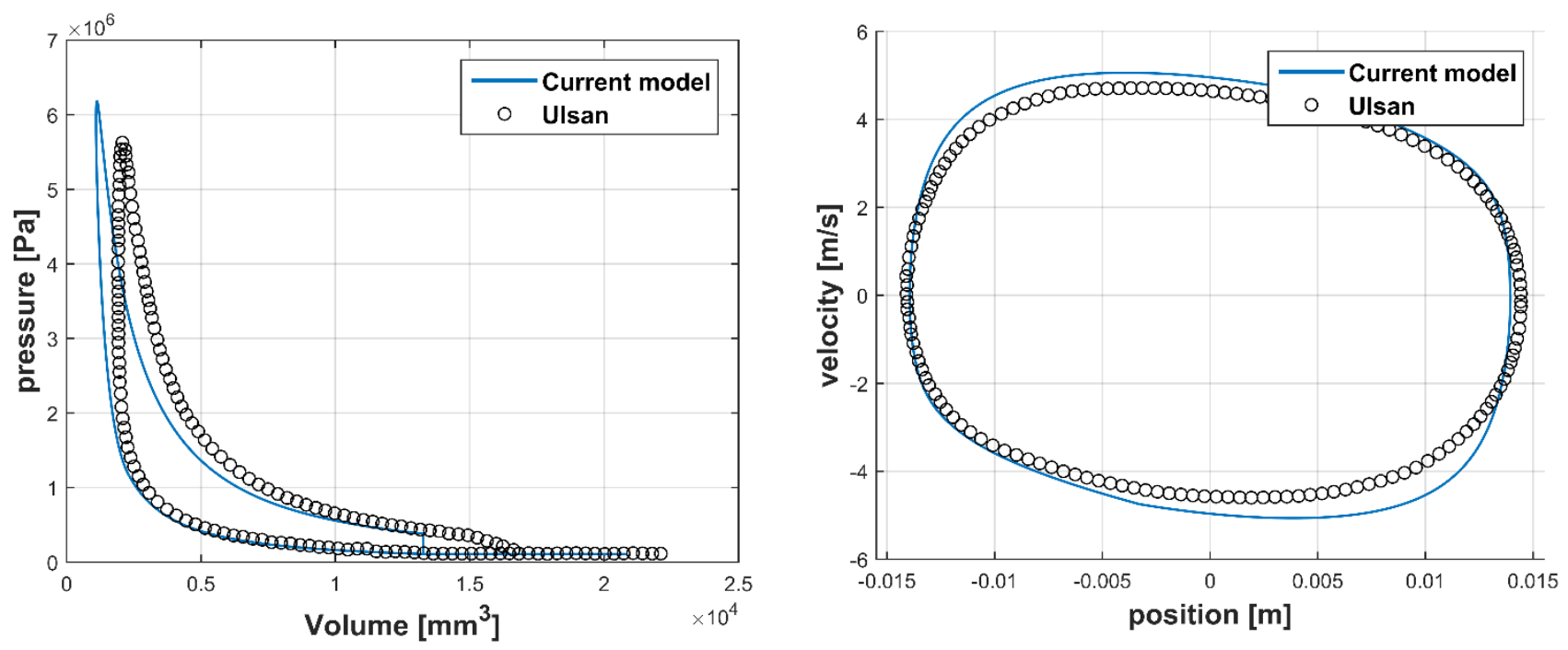

Figure 60: Comparison of simulation to University of Ulsan experimental data.

Second, the model from West Virginia University is compared with the current numerical simulation.

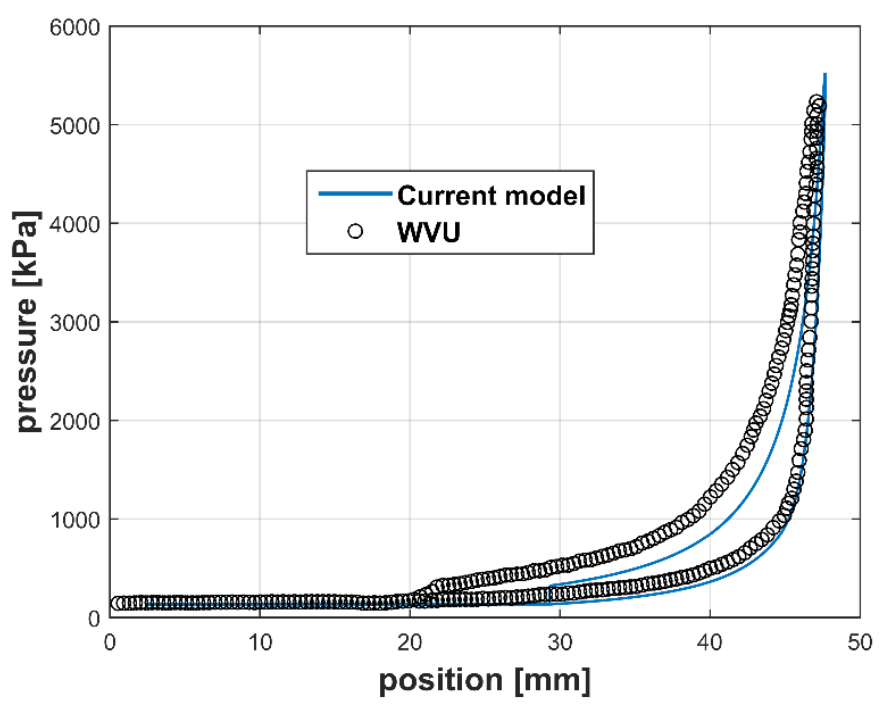

Figure 61: Comparison of current simulation to West Virginia University experimental data.

In terms of peak pressure magnitude and location, the numeric model is shown to better predict the performance of the WVU device than the Ulsan device. The CFR ignition model is used to predict ignition timing while the single Wiebe is used for combustion heat release profile. The numeric model suffers most from under-prediction of the expansion pressure profile which likely leads to the underprediction of stroke length: close scrutiny of the left turn around point shows the current model stroke ending sooner than the WVU prototype. The lower expansion curves are due the differences in 
combustion profile and duration. The rapid $\mathrm{HCCl}$ combustion is short compared to the flame front propagation burn of the spark ignited engines, causing the reduced expansion profile.

\subsection{Conclusions}

A detailed description of the numerical simulation model and its implementation is given along with sensitivity analysis and validation of the results from the model. Significant focus has been placed on development of the model because of its importance for the rest of this work. Additionally, construction of the model in Simulink affords substantial modularity for additions and alterations in support of future research.

From the sensitivity analysis, thresholds were given for time step and simulation time. By examining these and the solution method, it is shown that computational time can be minimized to around 2.5 seconds per simulation. Note that this is the time required after the model has been compiled and run as an executable rather than from the Simulink environment. Nonetheless, the build and run operations can be completed via MATLAB ${ }^{\circledR}$ script, and this feature is extensively used to accomplish the parametric study and optimization in the next chapter.

The choice of combustion heat release and heat transfer profile shape is shown to be of small importance. In fact, the shape of the flux curve is of much lesser importance than the total amount of energy added to or subtracted from the system. This is corroborated by Lee [78] concerning both friction and alternator models as well. Predicting the relative amounts of energy exchanged within the system represents the most significance as well as the most uncertainty.

Essential characteristics for accurate modeling are combustion duration and ignition timing. For $\mathrm{HCCl}$ combustion, these parameters can entirely shape the performance of the device (allowing that a stiff enough spring could be used to de-emphasize the effects of the cylinder pressures but not the overall energy balance). Two ignition delay models are presented as viable options and each are used in comparison with prototype device data. The RCM model tends to predict earlier combustion and tries to account for the effects of EGR while the CFR model has also been proven to adequately predict transient $\mathrm{HCCl}$ combustion. The data suggests that ignition control will be a key parameter to ensuring operational stability, and certainly efficiency. Although it has been claimed that FPLE's can adapt automatically in $\mathrm{HCCl}$ operation by increasing compression ratio until ignition occurs, the authority of this adaptation is limited. For the next chapters, CFR model is employed within the model, but the choice is somewhat arbitrary given the current state of free piston engine development and the amount of uncertainty involved in modeling other engine processes. For instance, constant values are assumed for combustion duration and efficiency, but are certainly subject to scrutiny and detract from the accuracy of the model. 
While the current investigation focuses on the use of this model to study the dynamics of the SALEG, the overarching achievement presented here is a robust modeling platform which can be passed on to support a wide variety of FPLE research. It is the author's expectation that experimental data will eventually be available for improvement of the simulation. Additionally, advanced computational methods (such as finite element analysis for the linear alternator or chemical kinetics calculations for the combustion process) could be integrated into the simulation or design routine to augment the predictive accuracy of the model. 


\section{CH. 4 PARAMETRIC STUDY AND OPTIMIZED DESIGN}

In the previous chapter, the numeric model was described and tested to show that the model presents valid responses for comparison of the simulated SALEG. This chapter examines the design space of the SALEG based on the numeric model. Data gathered from parametric sweeps are used to demonstrate trends and relationships between input settings and steady state behavior. Unique to this study is the integration of component design within the simulation.

While detailed component design is not within the scope of this investigation, practical design considerations must be taken into account for a parametric study of system design to have meaning. As an example, the total moving mass of the system has significant impact on performance (i.e. increased mass corresponds to lower frequency and reduced power density). To raise the frequency, the spring stiffness might be increased. However, raising the stiffness of a mechanical spring system also entails an increase of the total system inertia, implying a loss to the frequency. Many similar "give and take" examples can be seen as a result of the highly interdependent nature of the SALEG. It is the purpose of this chapter to explore the design space with respect to some practical design considerations and to gain important insights into the behavior of the SALEG.

\subsection{Parameter Definitions}

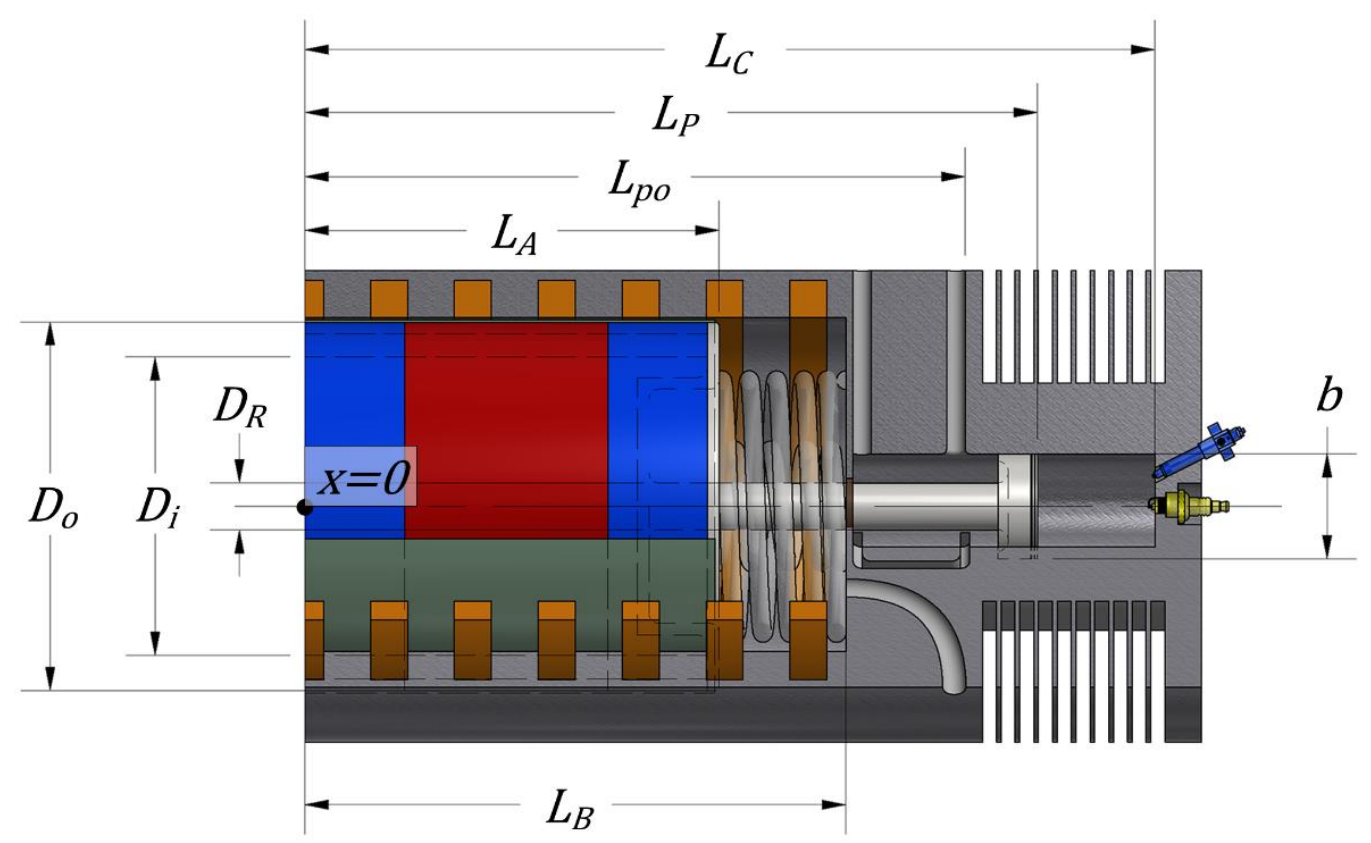

Figure 62: Illustration of the right half of the SALEG with primary dimensions included.

For this study, the primary dimensions of the SALEG are described in Figure 62. The illustration shows only the right half of the SALEG with the translator in its neutral position. In other words, with the 
free piston translator at its position in the figure (the midpoint position), the entire system is symmetric. The midpoint of the system is indicated at the $x=0$ location. All horizontal dimensions originate from the midpoint and all diameters are revolved around a horizontal axis running through the midpoint. Descriptions for each dimension are given in Table 7.

Table 7: Summary of the dimensions and their descriptions from the SALEG illustration in Figure 62.

\begin{tabular}{|c|c|}
\hline Dimension & Description \\
\hline$D_{o}$ & Outer diameter of alternator mover \\
\hline$D_{i}$ & Inner diameter of alternator mover \\
\hline$D_{R}$ & Diameter of translator connecting rod \\
\hline$b$ & Engine cylinder bore \\
\hline$L_{A}$ & Length of alternator mover from midpoint \\
\hline$L_{B}$ & Length from midpoint to inner rod bearing location \\
\hline$L_{p o}$ & Length from midpoint to ports opening location \\
\hline$L_{P}$ & Length from midpoint to piston crown \\
\hline$L_{C}$ & Length from midpoint to cylinder head \\
\hline
\end{tabular}

Each of these dimensions are related to practical design requirements. The alternator mover dimensions are dependent on alternator design calculations; the connecting rod diameter must meet structural design requirements; and the spring seat depth is a defining parameter for design of the compression springs. It is notable that significant literature is dedicated to designing the tubular permanent magnet linear alternator (e.g. [50, 51]). Mechanical spring design is a topic which might deserve similar attention. Design optimization for stiffness, size, weight, and fatigue life is difficult, and might be made more so by thermal influences as heat is transferred from the engine cylinders. Novel spring mechanism design, which might include everything from air springs to cantilever bars, could be the topic of multiple studies. This study considers only the simple case which involves the linear spring force relationship given in Eq. 41. Other practical design considerations encompass the horizontal dimensions given in Figure 62. The length of the alternator mover (as well as its inner and outer diameters) is defined by the steady state stroke length, engine speed, and power output according to [51] and given in Eq. 60 .

$$
D_{o}=\frac{P_{\text {out }}}{\pi m_{r} \tau f_{x r} \bar{v} \eta_{\text {alt }}}
$$

Eq. 60

The relationship in Eq. 60 shows the outer diameter $\left(D_{o}\right.$ in $\left.\mathrm{m}\right)$ as a function of power $\left(P_{\text {out }}\right.$ in W), number of poles $\left(m_{r}\right)$, pole pitch $(\tau$ in $\mathrm{m})$, specific thrust force $\left(f_{x r}=1.32 \cdot 10^{4} \mathrm{~N} / \mathrm{m}^{2}\right)$, mean translator speed $(\bar{v}$ in $\mathrm{m} / \mathrm{s}$ ), and rated alternator efficiency $\left(\eta_{\text {alt }}\right)$. For high efficiency in the range of $95 \%$, it is recommended to match the alternator design to the steady state operation of the translator [51]. So the pole pitch is set to equal the stroke and, along with mean velocity and power output, taken from steady state operation. This implies a feedback loop where steady state operation affects the alternator design which in turn affects 
steady state performance. The speed of the engine in cycles per second can be used to compute the mean velocity of the translator at steady state (Eq. 61).

$$
\bar{v}=2 \cdot \bar{S}_{S S} \cdot \bar{f}_{s S}
$$

Eq. 61

The mover diameter can now be mapped according to four parameters: stroke, frequency, power, and number of alternator poles. This map is given for a two pole design in Figure 63 which shows very large alternator diameters $(20 \mathrm{~m})$ are required to achieve high power output at low speed and short stroke. Alternator diameter decreases exponentially with increases in frequency and stroke. By Eq. 60, as the number of poles increases, the diameter of the alternator decreases. In contrast, the length of the alternator increases with the increase of poles, and the real importance of this discussion is the alternator moving mass which is a function of both alternator length and diameter. For a more rigorous design process, the optimal magnet height would be found via the magnet volume for a given flux density with finite element analysis tools. Such an endeavor is beyond the scope of this investigation, so the inner diameter of the magnets is assumed to be $80 \%$ of the calculated outer diameter. For a constant material density and with known length, the volume and mass of the alternator mover is available.

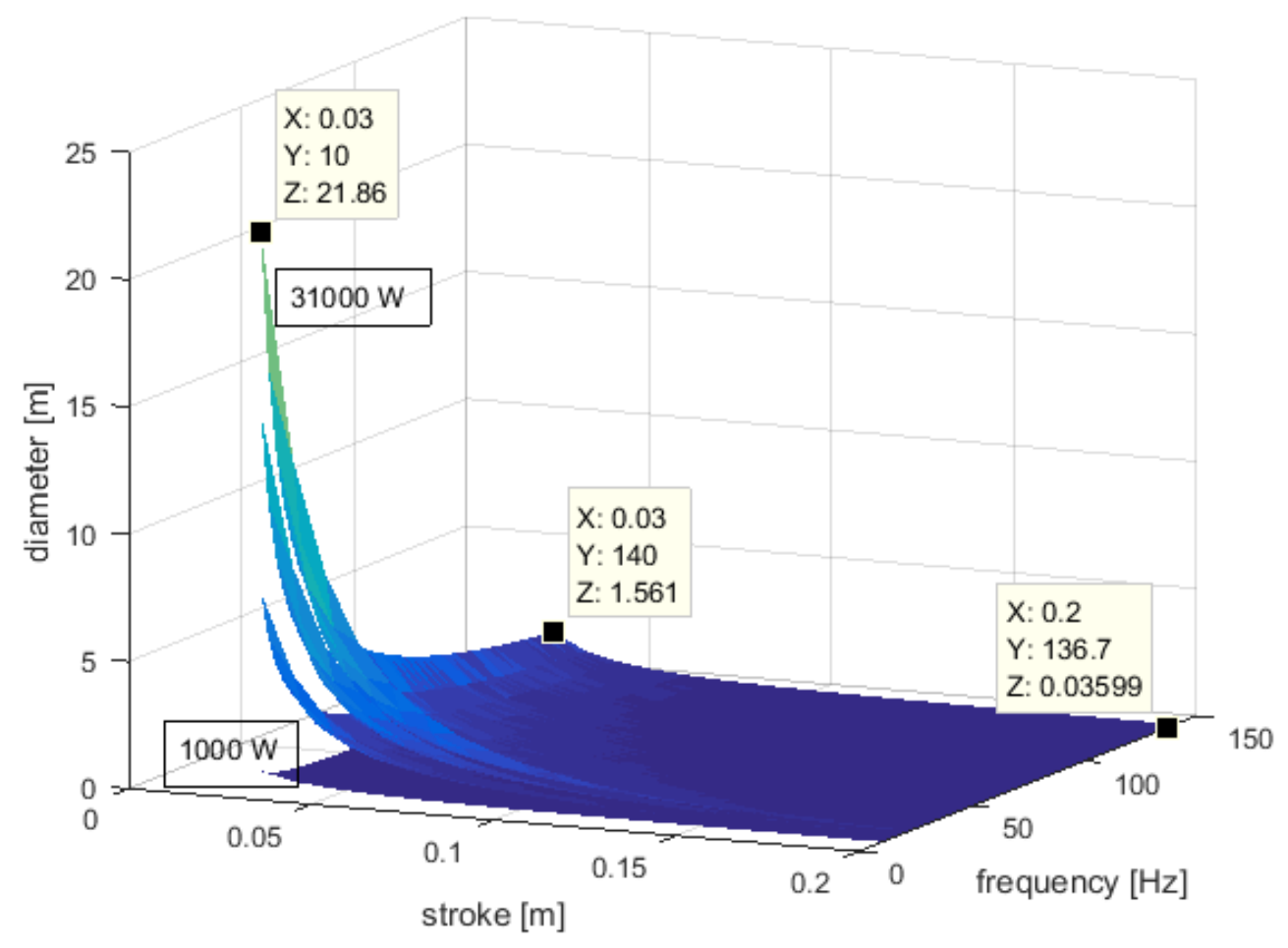

Figure 63: Alternator parameter map for a two pole design based on Eq. 60. Points are given for reference where $X$ indicates stroke, $Y$ indicates frequency, and $Z$ indicates diameter.

Assuming a two pole design (which differs from that shown in Figure 62), the length parameter $L_{A}$ must be equal to length of the stroke $\left(L_{A}=S_{S S}\right)$. To avoid clearance issues, the space between the bearing location $\left(L_{B}\right)$ and the alternator is set equal to the piston and cylinder neutral clearance length (Eq. 62). 


$$
L_{C}-L_{P}=L_{B}-L_{A}=L
$$

The neutral clearance length was previously given in Eq. 1, and it remains to be one of the defining parameters of the system as the maximum stroke is equal to twice the natural clearance length. Bearing and piston skirt lengths are assumed to equal a total of $14 \mathrm{~mm}$, but it is noted that bearing design for minimal friction deserves the attention of a separate design study. To accommodate the opposite piston and cylinder geometry, the 'crankcase' space beneath the piston must also have a neutral clearance of length $L$. With these constraints, the length from the midpoint of the alternator mover to the piston crown can be related to a previously defined term (Eq. 63).

$$
L_{P}=L_{B}+0.014 m
$$

Eq. 63

The length of the piston is important for calculation of the rod mass and for parameterizing the port location. The port opening location is parameterized according to the theoretical limits of the translator motion. If the piston shown in Figure 62 were moved to its leftmost limit (achieving the maximum clearance between piston and cylinder head), the port opening value at the piston crown location would correspond to zero. Conversely, a parameterized value of one for the port location corresponds to the point at which the piston crown contacts the cylinder head. The subscripted percentage in Eq. 64 corresponds to the port location parameter which must be in the domain $(0,1)$.

$$
L_{p o}=L_{p o \%} \cdot(2 \cdot L)+\left(L_{P}-L\right)
$$

Eq. 64

Now that the diameter and length parameters are defined, the mass of the translator can be represented. Without a rigorous design effort, assumptions are made to complete the calculations. Starting with the piston head, an increase of piston bore corresponds to an increase of mass.

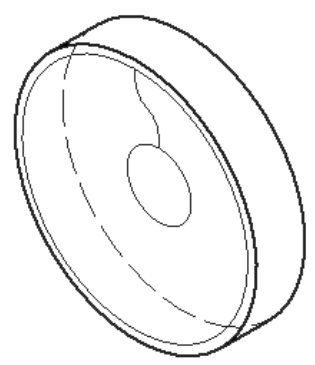

Figure 64: Illustration of the basic piston head design. The underside of the piston head is shown in the forefront and the crown of the piston head is assumed to be a flat surface.

Assuming a fixed skirt length of $8 \mathrm{~mm}$, basic design (Figure 64), and material density $\left(7800 \mathrm{~kg} / \mathrm{m}^{3}\right)$, mass data can be gathered as bore is varied. A second order polynomial relationship is formed to relate the mass of the piston heads to the bore. The relationship is shown in Eq. 65 and its plot follows in Figure 65. 


$$
m_{\text {bore }}=82.3 \cdot b^{2}+0.0136 \cdot b-8.16 \cdot 10^{-5}
$$

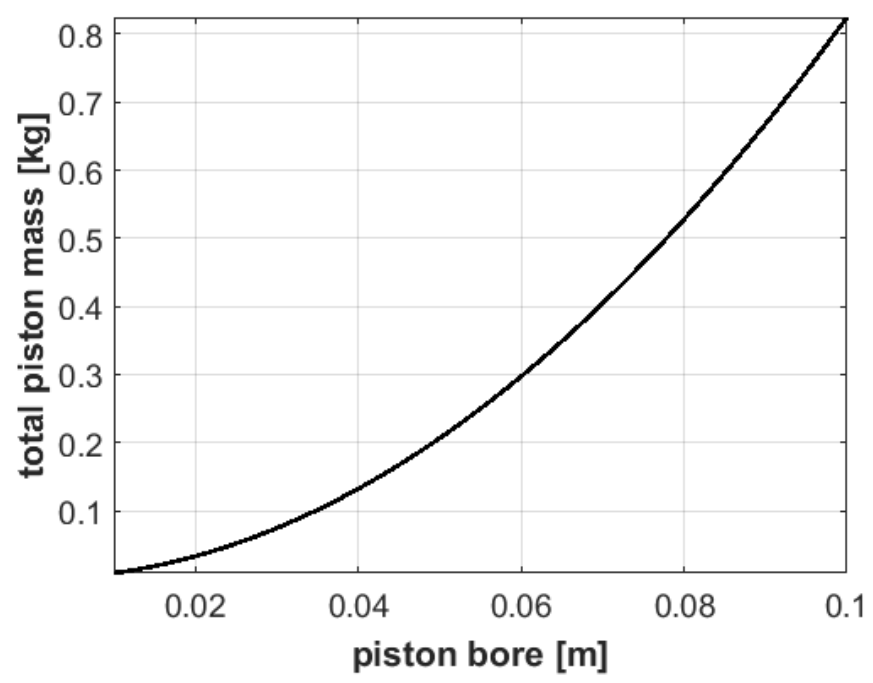

Figure 65: Quadratic relationship for the mass of piston heads dependent on bore diameter.

It is assumed that the translator connecting rod extends from beneath the right piston head, through the alternator mover, to beneath the left piston head. Practically, the cross sectional surface area of the rod must withstand the maximum acceleration and associated dynamic stresses that could lead to mechanical failure. The rod also acts as the bearing surface for the translator, so thermal effects from friction and heat conduction from the piston head are worth investigation. In short, the mass of the translator should be minimized while maintaining safe performance. The geometry of the translator does not lend itself to simple analysis, so a fixed cross sectional area of $75 \mathrm{~mm}^{2}$ is assumed for the full length of the translator rod. With constant material density (same as that given for the piston heads), the mass of the rod $\left(m_{\text {rod }}\right.$ in $\mathrm{kg}$ ) scales proportionally with its length $\left(L_{P}\right.$ in $\left.\mathrm{m}\right)$ by Eq. 66.

$$
m_{\text {rod }}=0.585 \cdot 2\left(L_{P}-0.008\right)
$$

Eq. 66

Spring design was mentioned as a complicated endeavor with many possible paths. This investigation cannot deal with such complexity within the simulation, but it is important to recognize a relationship between spring stiffness and total moving mass. Thus, a linear relationship is formed to represent the mass penalty associated with increased spring stiffness (Eq. 67) where the mass in kilograms is calculated with respect to spring stiffness in Newtons per meter.

$$
m_{\text {springs }}=\frac{10}{350000} k
$$

Recognizing that the natural frequency of an oscillating system is strongly dependent on $\sqrt{k / m}$, the frequency of the free piston engine is directly affected by Eq. 67. These effects are demonstrated in Figure 66. 


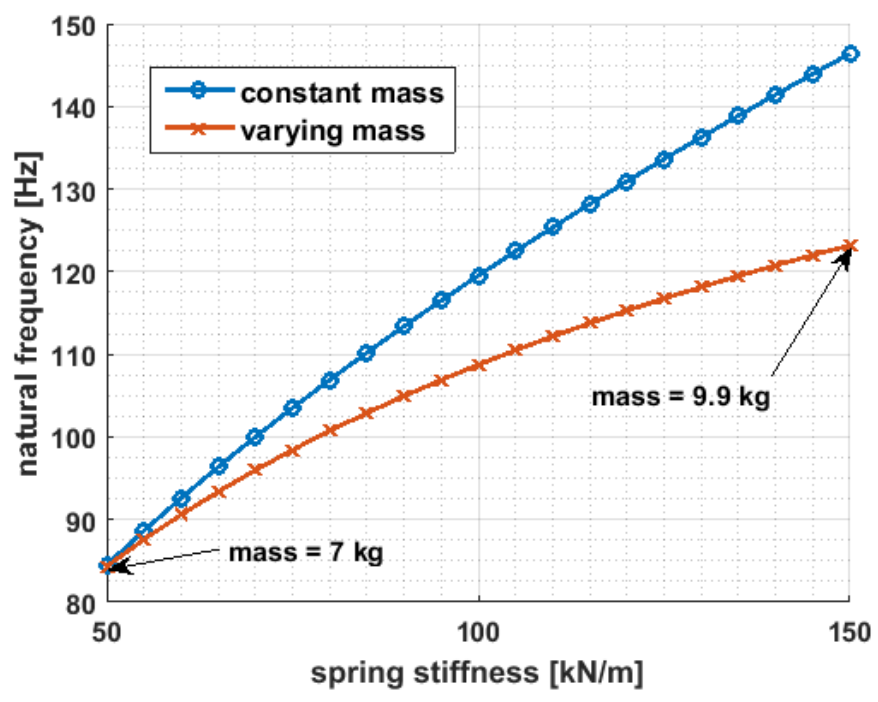

Figure 66: Comparison between natural frequency increase with and without the inclusion of spring inertia variation.

Separation between the case with constant mass and the varying mass case reinforces the importance of spring design. While increasing the frequency of the engine may lead to better performance and may be accomplished by raising the spring stiffness, the increase of inertia must be managed to maintain the effectiveness of the spring design. Furthermore, the relationship between spring stiffness and moving mass is not as simple as the linear relationship given in Eq. 67. For true spring design, system geometry and operation will play important roles.

Aside from the geometric and mass relationships given, additional independent parameters are used to define the intake conditions and engine cylinder characteristics. The eight total independent parameters under consideration are summarized in Table 8 with descriptions for each.

Table 8: Summary of independent parameters used to define the SALEG steady state design simulation.

\begin{tabular}{|c|c|}
\hline Symbol & Description \\
\hline$b$ & engine cylinder bore \\
\hline$L$ & maximum half stroke $\left(L_{C}-L_{P}\right)$ \\
\hline$L_{p o \%}$ & location of ports given as percentage of allowable \\
\hline$k$ & spring stiffness \\
\hline$P_{i n}$ & intake pressure \\
\hline$T_{\text {in }}$ & intake temperature \\
\hline$\phi$ & equivalence ratio \\
\hline$E G R$ & exhaust gas retained as percentage \\
\hline
\end{tabular}

Aside from the input parameters, primary system outputs and measures of performance must also be defined. System efficiency is defined as the amount of fuel energy converted to alternator power after losses occur due to combustion and alternator inefficiencies, adverse work around top dead center, heat transfer, friction, and heat rejection. Mean power output by the alternator, peak cylinder pressure, compression ratio, stroke, frequency, and designed translator mass provide a quantitative view of the 
system. Finally, cycle stability is measured as the change in translator start and stop locations after a full cycle, normalized by the system length geometry $(L)$. This is shown in Eq. 68 where $x_{1}$ and $x_{2}$ correspond to the start and stop positions of the translator, respectively.

$$
\text { stability }=\frac{\left|x_{1}-x_{2}\right|}{L}
$$

For the following parametric study and optimization, these eight inputs and outputs are the focus for understanding the nature of the SALEG.

\subsection{Effects of Primary Parameters}

The eight parameters given in Table 8 do not include all of the parameters that could be used to define the system (such as spring or alternator design parameters, variations in fuel characteristics, or more specific port or cylinder geometry). They do, however, represent the primary defining parameters of the system and bring to bear a large multi-dimensional design space. This parametric study begins with individual parameter sweeps stemming from the Ch. 3. base case. Starting with bore, Figure 67 demonstrates the effect of bore on system stability.

Again, stability is measured by the difference between translator start and stop positions for the final cycle of simulation where each simulation is stopped after 2.5 seconds of simulated time or if the piston contacts the cylinder head. The stability calculation is then normalized by the neutral clearance length so that zero corresponds to perfect stability. This is not the only possible measure of stability. The cycle-to-cycle variation of stroke length is a similar measure. A weakness in either approach is that the stop time (discussed in section 3.6.2 as the time within the simulation space that the simulation is stopped) is apart from the translator behavior. In other words, the simulation could end at any point during a cycle if the stop time is reached. Furthermore, for two different simulations, the stop time will likely cause each simulation to end at different points along the respective cycle. Based on the sensitivity analysis in section 3.6.2, the selected stop time ensures that steady state behavior is reached if possible. However, for an unstable simulation, the repeatability of the stability calculation is low if the stop time is varied. For the following parametric study, stability is viewed within the context of regions of unstable behavior and with respect to complimentary indications such as excessively high compression ratio. The change in dead center position has proven to be a sufficient measure of stability for this parametric study and the following optimization. In the final chapter, the dead center position is shown to be useful as a mechanism for control. 

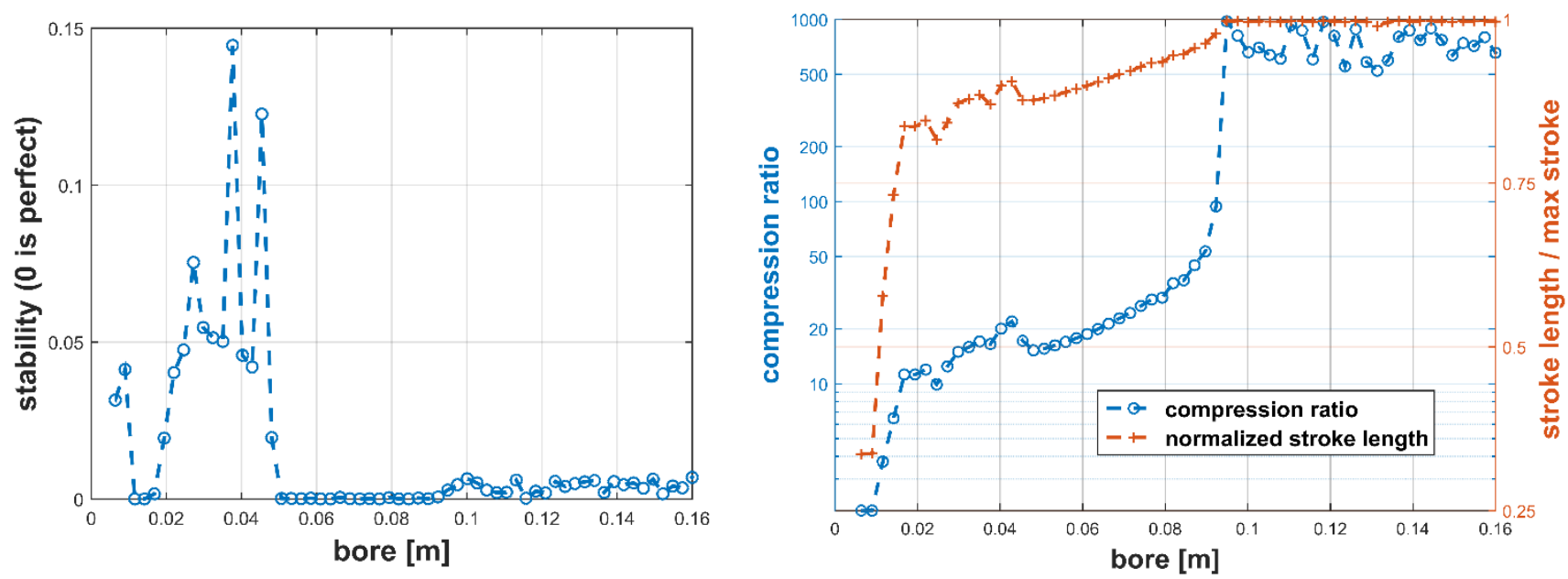

Figure 67: Parametric sweep of bore showing its effects on compression ratio, stroke, and stability.

In Figure 67, the left-hand plot shows stability while the right-hand plot shows compression ratio and normalized stroke length, all as functions of bore. For the base case, bore was equal to $64 \mathrm{~mm}$. Demonstrated in the stability plot is a central region where stability is consistent and then two outer regions where unstable behavior arises. Instability is particularly high in the left region when bore drops below a threshold, in this case about $50 \mathrm{~mm}$. The left-hand unstable region corresponds to erratically decreasing compression ratio and stroke length in the right-hand plot, eventually falling off as compression falls below ten and combustion is no longer achieved.

Instability is related to the relative cylinder energy. Reducing the cylinder bore results in lower cylinder energy. In the lower unstable region, cylinder energy is sufficiently reduced so that stable oscillation cannot be maintained. For the simulation cases between about 20 and $40 \mathrm{~mm}$ cylinder bore, ring-down of the translator is prevented within the simulation space by the alternator controller and integrated component design calculations. The 'transient' nature of these solutions results in the instability.

Comparing the stability plot to the compression and stroke length plot, the stable region coincides with smooth increases of compression ratio and stroke length. Through the stable region, increasing bore leads to higher system energy and higher compression ratio. The stable simulations in this region reflect negligible cycle-to-cycle variations, resulting from balanced energy exchange within the system.

The lack of stability in the right-hand region is shown to coincide with unrealistically high compression which increases by a factor of ten from 50 when the bore exceeds about $80 \mathrm{~mm}$. These high compression cases are the result of excess cylinder energy that leads to runaway compression events beyond the ability of the system to dissipate energy through friction, heat transfer, and the 
electromagnetic resistance of the alternator. The ability of the small alternator controller to increase load is purposefully limited to reduce its effect on steady state performance.

The excessive compression shown in Figure 67 corresponds to peak pressures of greater than 250 bar in the right-hand plot of Figure 68. The current model does not include material limitations, and therefore knows no limit to in-cylinder pressure. In the same figure, peak pressure, power density, frequency, and efficiency increase almost linearly through the stable region. Power density is calculated as the ratio of steady state power output to designed translator mass and is considered a reasonable surrogate for power density.
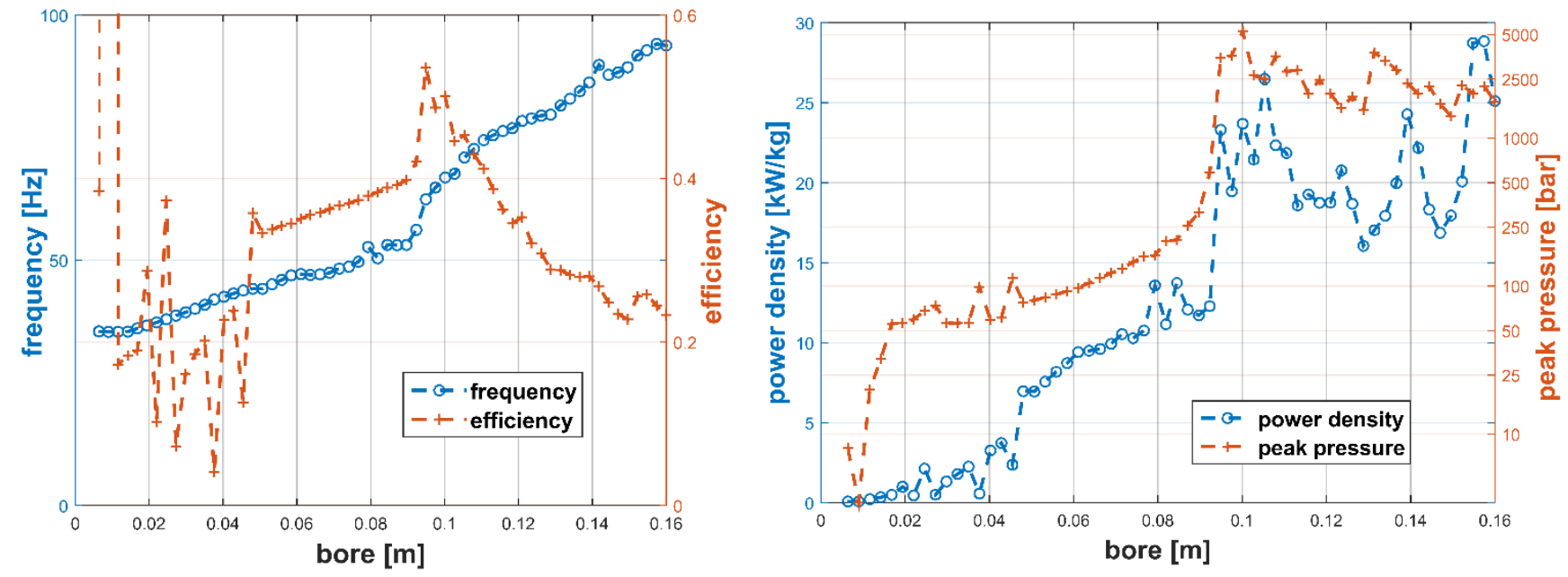

Figure 68: Parametric sweep of bore showing its effects on frequency, efficiency, power density, and peak pressure.

Through the stable region, efficiency rises linearly as a function of bore. The rise in efficiency is enabled by the rise in compression ratio, and overcomes the increased heat transfer losses that are associated with greater in-cylinder surface area. This is substantiated by the behavior in the upper unstable region. Despite the unrealistically high compression ratio, the compression ratio remains generally constant as bore continues to increase. Meanwhile, the increasing in-cylinder surface area allows for greater heat transfer and decline in efficiency. The insights discussed here related to energy balance are similarly demonstrated throughout the next parametric sweeps. Next, variation of allowable stroke length is considered with all other parameters kept constant according to the base case. 

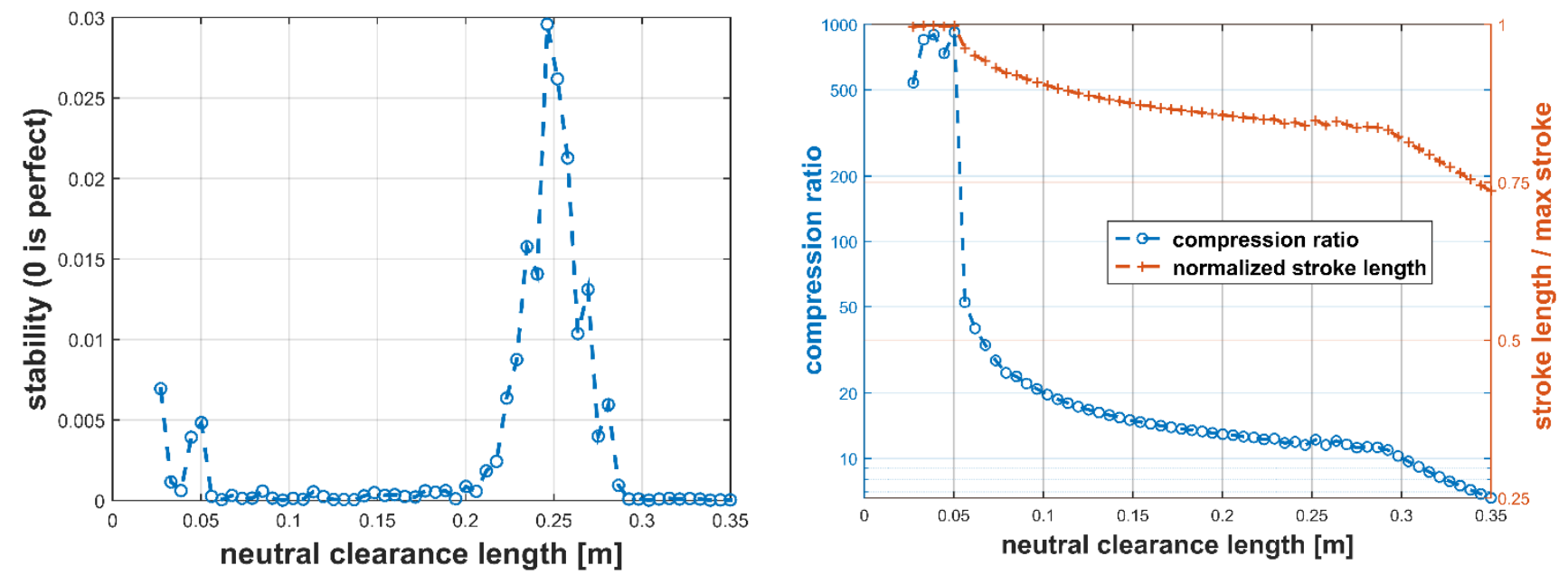

Figure 69: Parametric sweep of neutral clearance length showing its effects on compression ratio, stroke, and stability.

Figure 69 demonstrates the effects of the neutral clearance length on stability, compression ratio, and stroke length. Recall that the neutral clearance length is the space between the piston crown and cylinder head when the translator is at its midpoint position. Raising the neutral clearance length is equivalent to raising the maximum stroke. It is helpful to recognize again that the steady state stroke length is normalized by the maximum possible stroke even while the maximum stroke parameter is varied. This ensures unbiased comparison between solutions as the neutral clearance length is increased. The base case neutral clearance length is $100 \mathrm{~mm}$.

System stability is mostly held through the range of clearance values but falters as the clearance drops below $50 \mathrm{~mm}$. Additionally, a peak of instability arises in the region around $250 \mathrm{~mm}$. Further investigation reveals that the instability is caused not by an uncontrolled rise in expansion or ringing down, but is rather a case of rhythmic stability (showing a beat frequency). Rhythmic stability is not applicable within this steady state discussion due to the integrated component design routines. However, it is notable that the unstable behavior is manifested as the compression ratio falls near ten, similar to the unstable threshold seen previously when bore was varied. As the neutral clearance is raised past the $300 \mathrm{~mm}$ mark, natural steady state solutions are again found, though the decline in compression ratio and stroke length becomes more rapid. Comparing these plots with efficiency and power density seen in Figure 70, it becomes evident that the simulations with clearance length above $300 \mathrm{~mm}$ are 'ringing down' and will eventually come to rest.

Aside from stability concerns, raising the maximum stroke (clearance length) decreases compression ratio and stroke length. These effects are primarily the result of greater in-cylinder surface area and thus greater heat transfer loss. In line with the impact on compression, maximum stroke is shown to also effect efficiency. The lowest stable clearance length corresponds to efficiency greater than 
$40 \%$ and the lowest to below $20 \%$. For power density, a maximum is seen (considering only the stable region) around $135 \mathrm{~mm}$. For an extended view of the combined effects of bore and maximum stroke, the interested reader can refer to Figure 117 and Figure 118 in Appendix C.
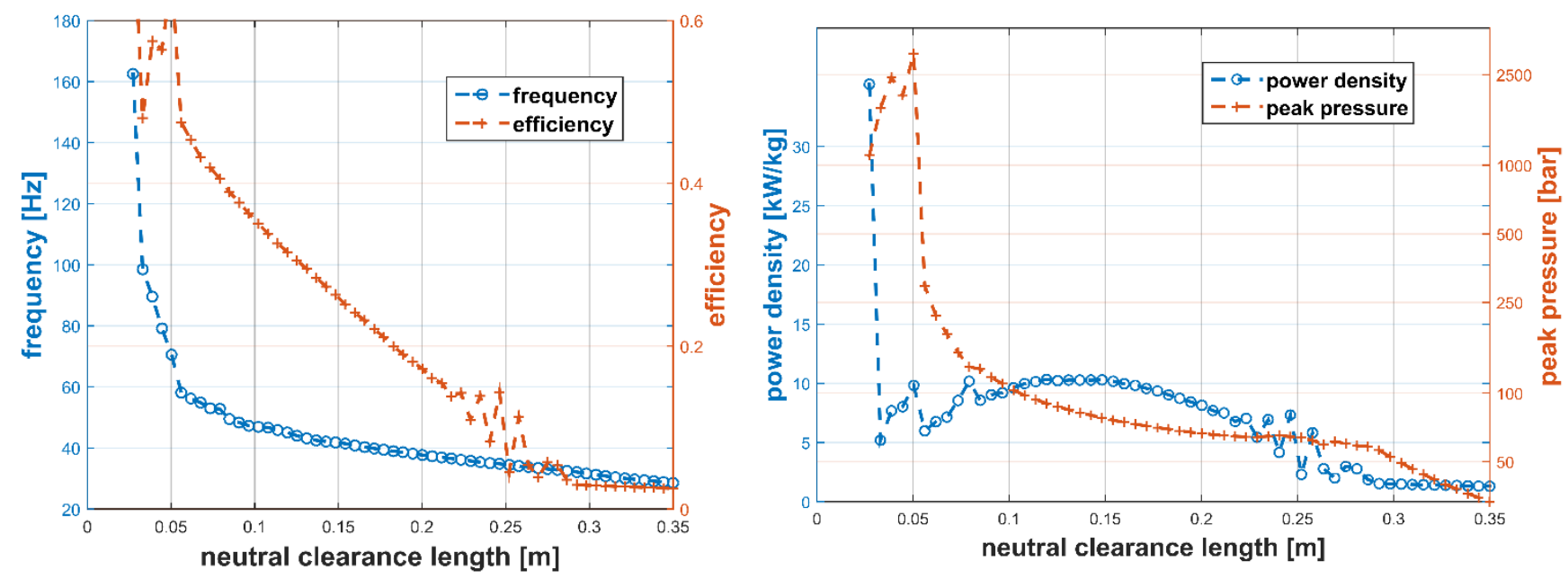

Figure 70: Parametric sweep of neutral clearance length showing its effects on frequency, efficiency, power density, and peak pressure.

During the design phase, port geometry is an essential design element that defines the gas exchange process. Unfortunately, the simplification of the gas exchange process undermines the usefulness of the parametric sweep through port location, but Figure 71 and Figure 72 demonstrate that the port location is bounded. The left bound - corresponding to a port location very near bottom dead center minimum - is due to the inability of the piston to reach a location with the port open. The right boundary indicates port placement that does not allow sufficient compression for ignition. Otherwise, moving the port location towards the cylinder head leads to decreases in frequency, compression ratio, power density, and peak pressure, but has negligible effect on efficiency. While these are consistent with the implied reduction of trapped cylinder volume (thus reduced cylinder energy), the true effects are uncertain due to the likely yet unspecified variations of cylinder pressure, temperature, and mixture associated with port location and geometry. 

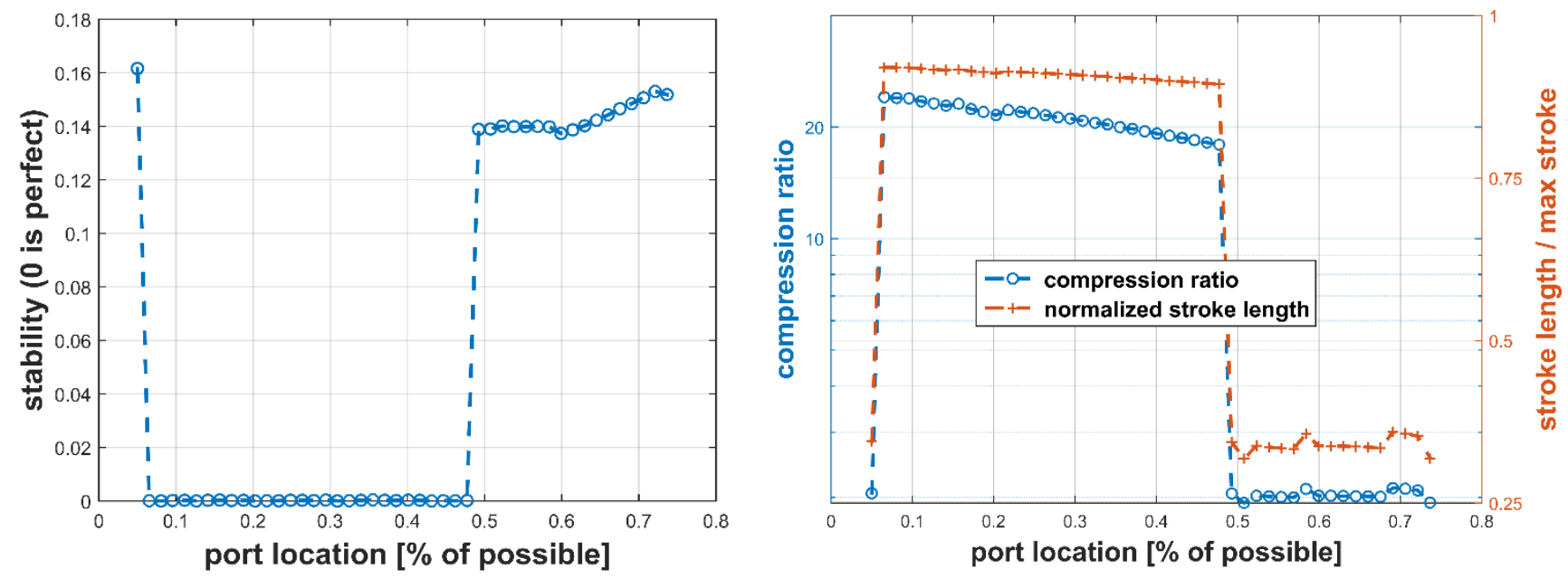

Figure 71: Parametric sweep of relative port location showing its effects on stability, compression ratio, and stroke length.
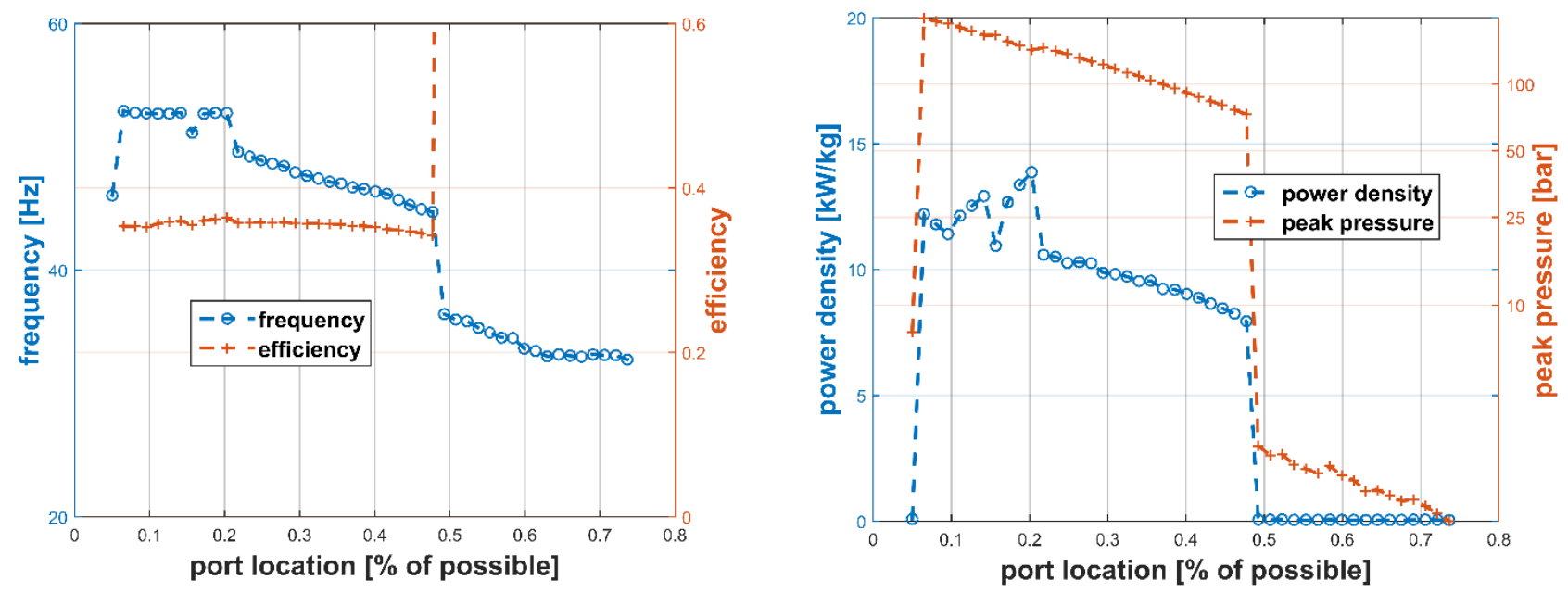

Figure 72: Parametric sweep of relative port location showing its effects on frequency, efficiency, power density, and peak pressure.

Next are considered the effects of intake pressure and temperature with pressure leading the discussion. Figure 73 shows the effects of raising the intake pressure on stability, compression ratio, and stroke length. Consistent throughout both Figure 73 and Figure 74 (showing the effects on frequency, efficiency, power density, and stroke length) is the intake pressure limitation around two bar. Above two bar, high cylinder energy leads to runaway compression and instability. It is notable here that intake pressures above about 1.5 bar result in peak cylinder pressures that may not be permissible considering material constraints. 

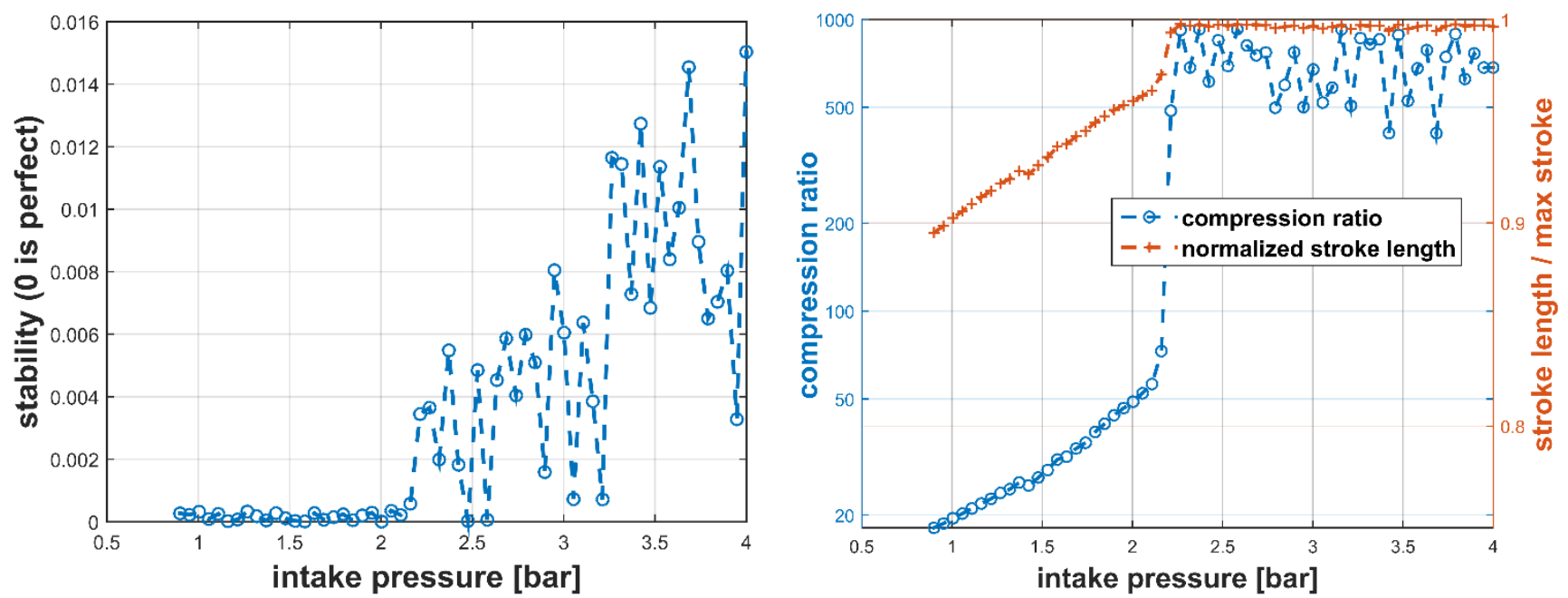

Figure 73: Parametric sweep of intake pressure showing its effects on stability, compression ratio, and stroke length.

Within the stable region, raising the intake pressure results in increases for all performance characteristics, pointing towards the possible benefit of a boosted intake system. Practically speaking however, the inclusion of boost may add cost and complexity and detract from overall system efficiency and power density by requiring additional system components.
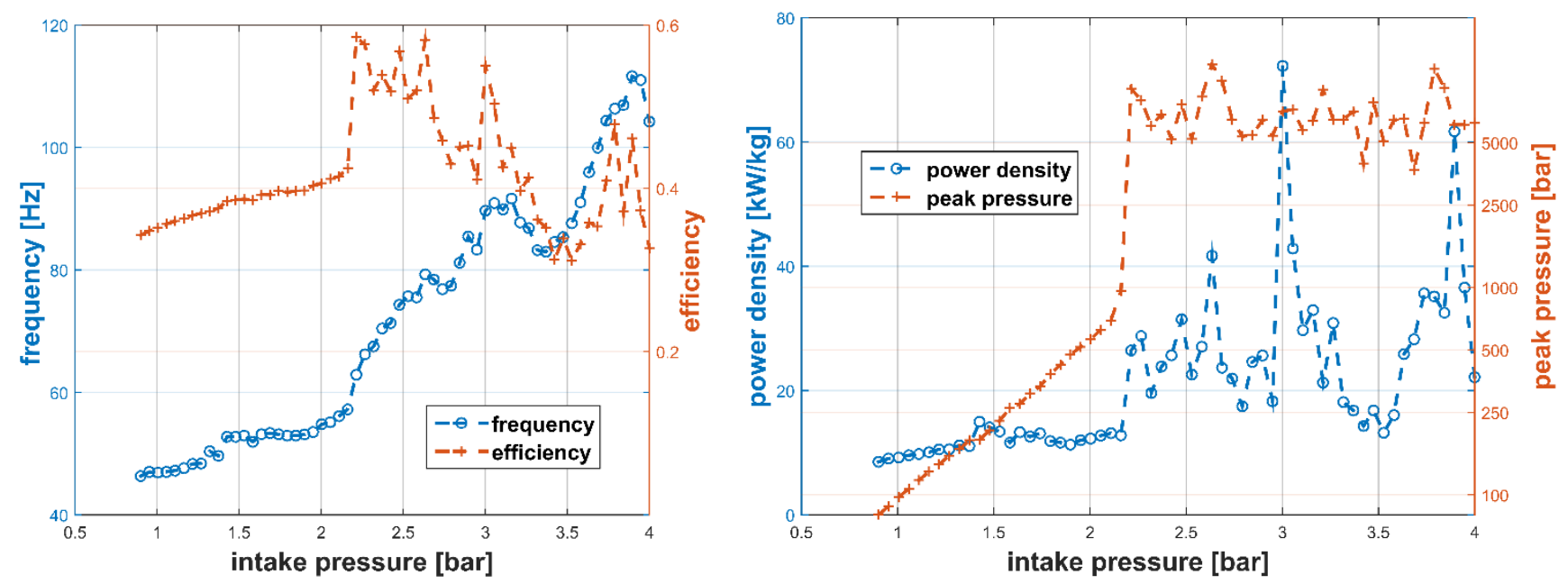

Figure 74: Parametric sweep of intake pressure showing its effects on frequency, efficiency, power density, and peak pressure.

The effects of intake temperature are given in Figure 75 and Figure 76 . Similar to the effects of intake pressure, stable operation is seen for increasing intake temperature but is limited by an upper bound. Dissimilar to intake pressure, increasing intake temperature is seen to cause decreases of compression, stroke length, efficiency, power density, and peak pressure. These decreases are due to the impact of temperature on start of combustion and inverse relationship between temperature and cylinder mass (according to ideal gas law). As intake temperature increases, combustion timing is 
advanced with respect to TDC. Early combustion timing combined with heat transfer has been shown to cause an inversion of the pressure-volume profile that causes adverse work while turning the piston around. The negative work then leads to lower compression and efficiency loss. The shortened stroke allows for higher frequency operation. It is important to note that the wall temperature is assumed to be $550 \mathrm{~K}$ through all parametric simulations. This is not consistent with steady state operation when intake temperature is raised. A more robust model for wall temperature should be pursued in future research to ensure the accuracy of these findings.
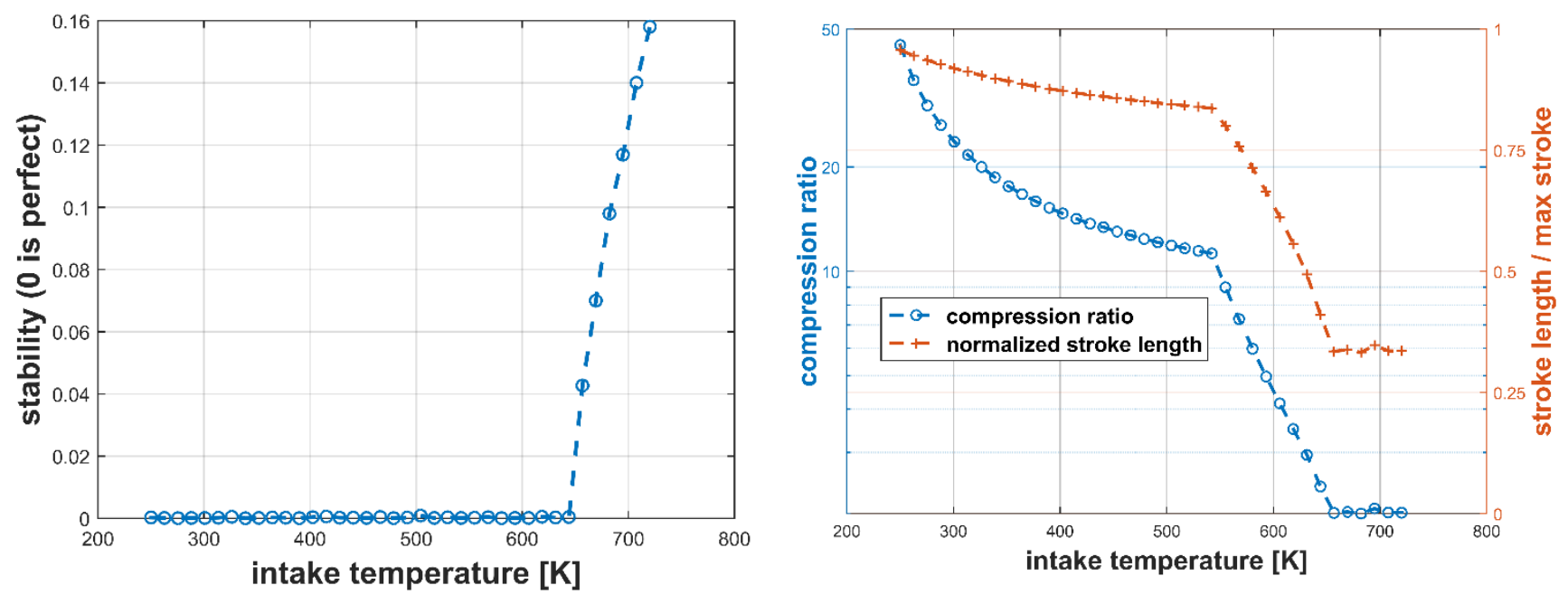

Figure 75: Parametric sweep of intake temperature showing its effects on stability, compression ratio, and stroke length.
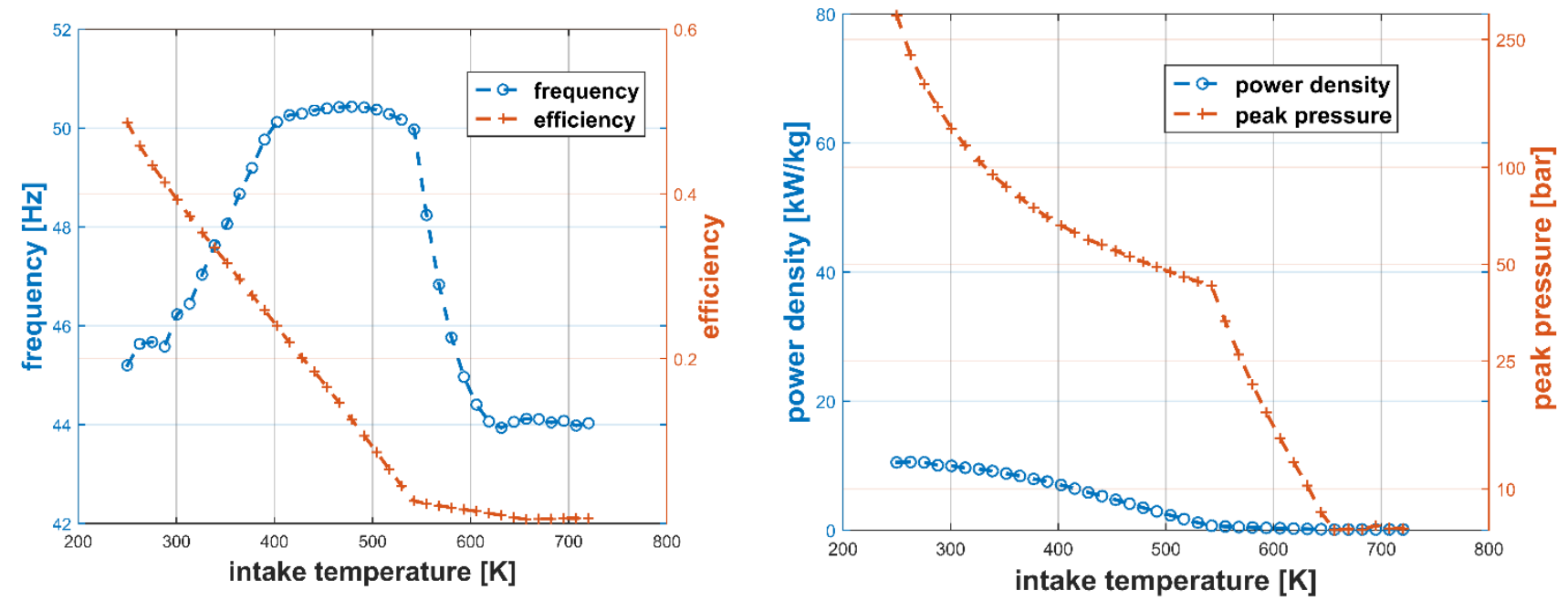

Figure 76: Parametric sweep of intake temperature showing its effects on frequency, efficiency, power density, and peak pressure.

Significant to the SALEG is the application of mechanical springs to support cyclic stability and increased frequency of the free piston engine. Previously it was shown that including a fraction of the 
spring mass in the total moving mass can have negative consequences on system frequency compared to the case where spring mass is neglected (via the natural frequency discussion around Figure 66). Figure 77 and Figure 78 demonstrate that while stability is ensured with spring stiffness greater than 50 $\mathrm{kN} / \mathrm{m}$, continuing to raise the stiffness results in decreased performance. The most significant loss in performance is in power density, where increasing spring mass contributes to the loss in conjunction with lowered frequency, while slightly lowered compression is tied to small losses in efficiency and peak pressure.
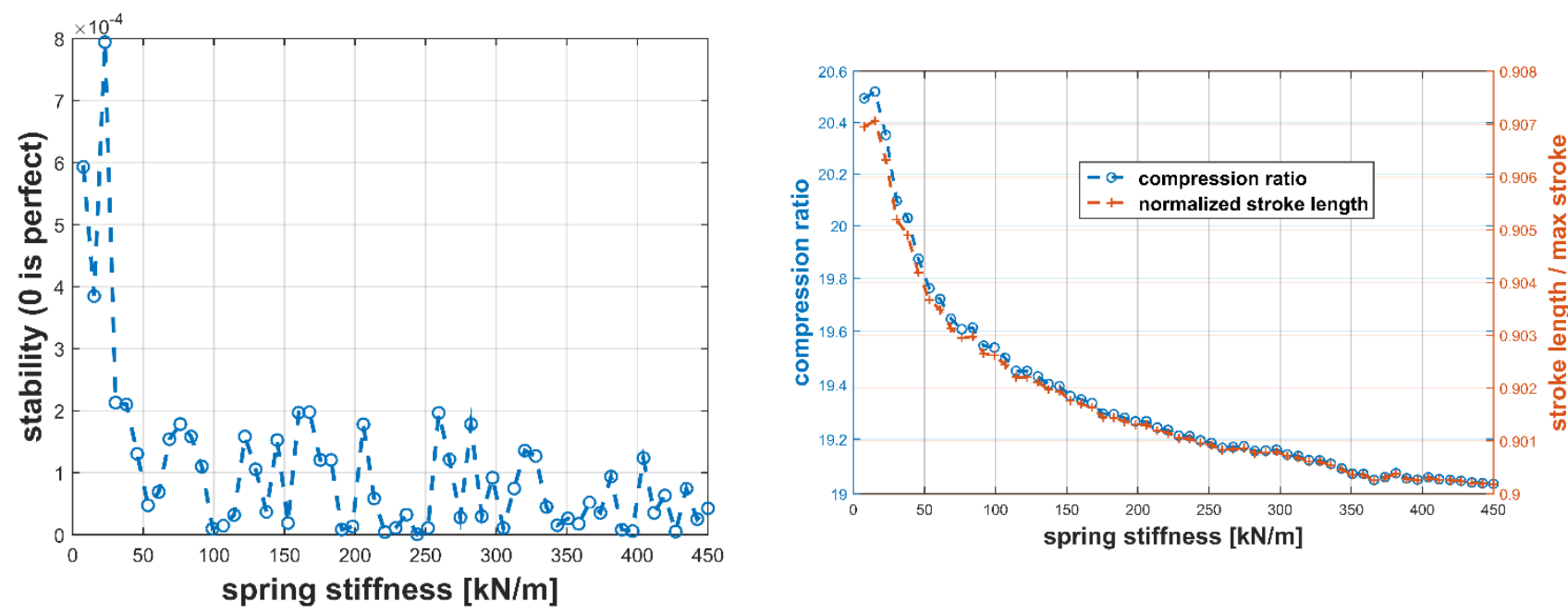

Figure 77: Parametric sweep of spring stiffness showing its effects on stability, compression ratio, and stroke length.
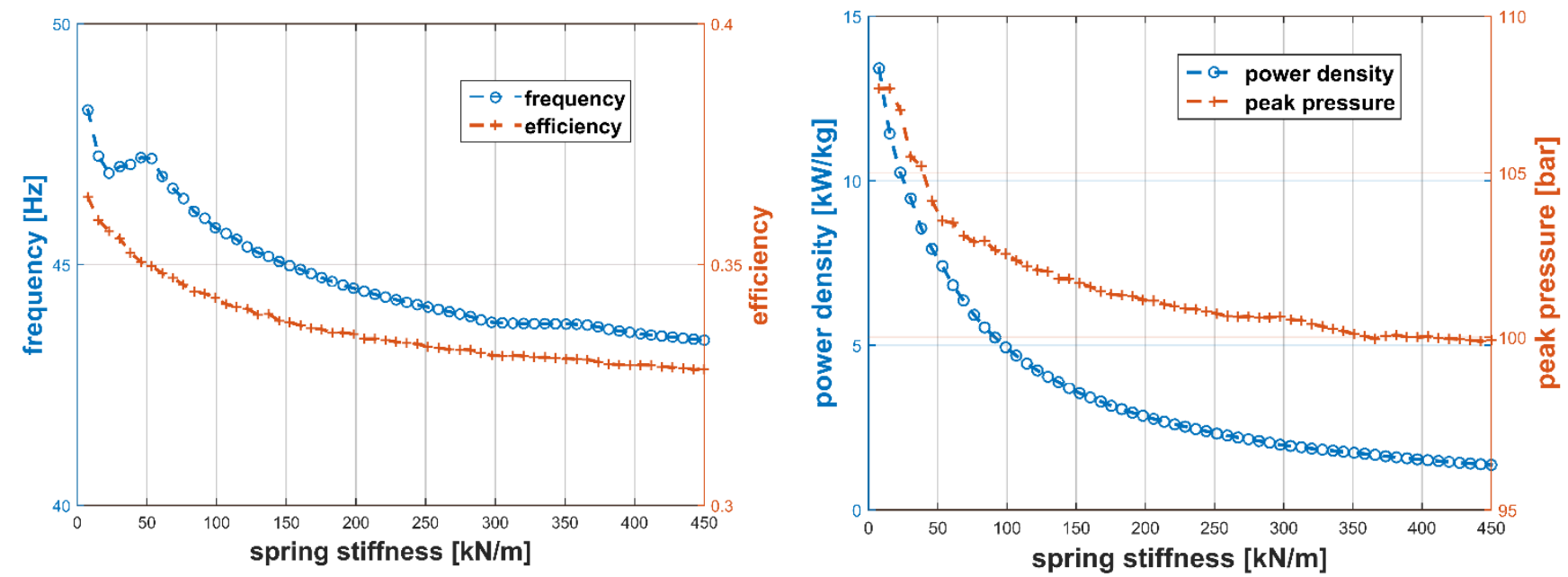

Figure 78: Parametric sweep of spring stiffness showing its effects on frequency, efficiency, power density, and peak pressure.

Operating in the ultra-lean $\mathrm{HCCl}$ combustion regime was discussed in the literature review as having high potential when combined with the free piston engine. While this investigation cannot cover the effects of equivalence ratio on the combustion process with any real detail, the high level effects of 
equivalence ratio are demonstrated in Figure 79 and Figure 80. For the given range of equivalence ratio, stability is consistent until the ratio nears a threshold around 0.2. Otherwise, rising equivalence ratio shows a positive influence on all performance factors. The responses for stroke length and peak pressure are linear while the ability to raise frequency and power density are limited after around 0.6.
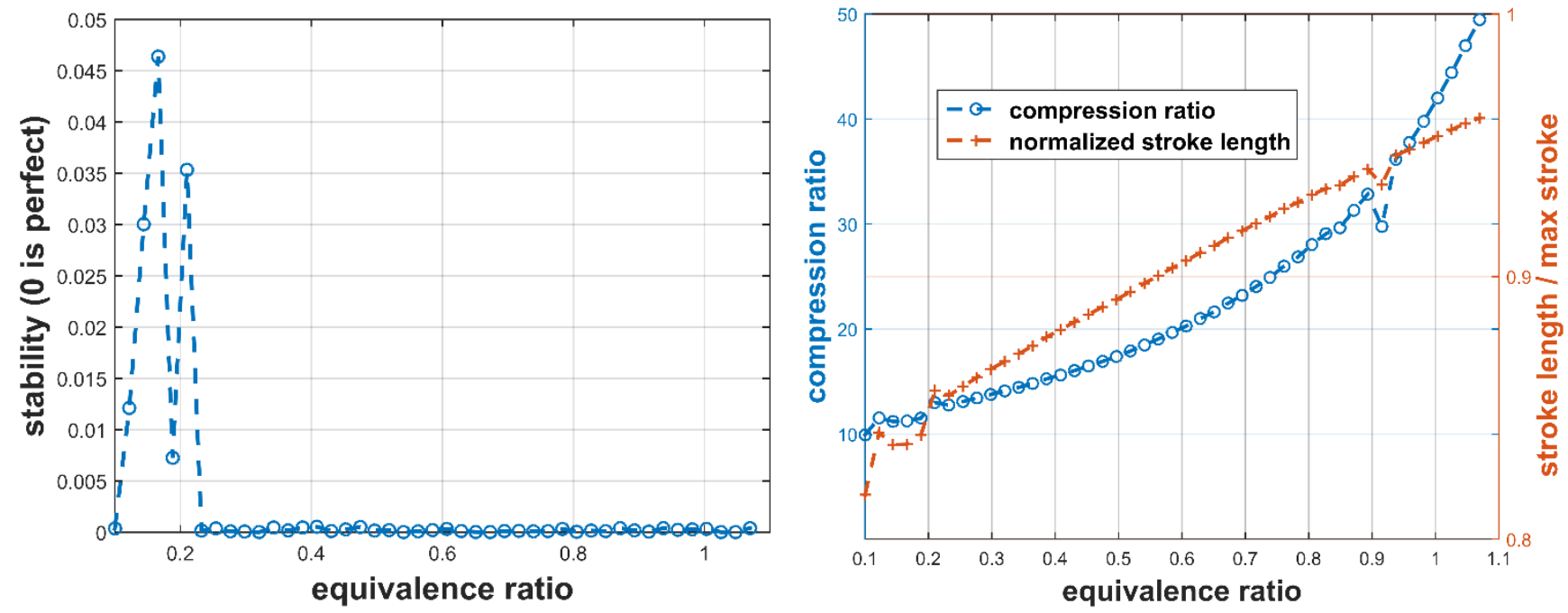

Figure 79: Parametric sweep of equivalence ratio showing its effects on stability, compression ratio, and stroke length.
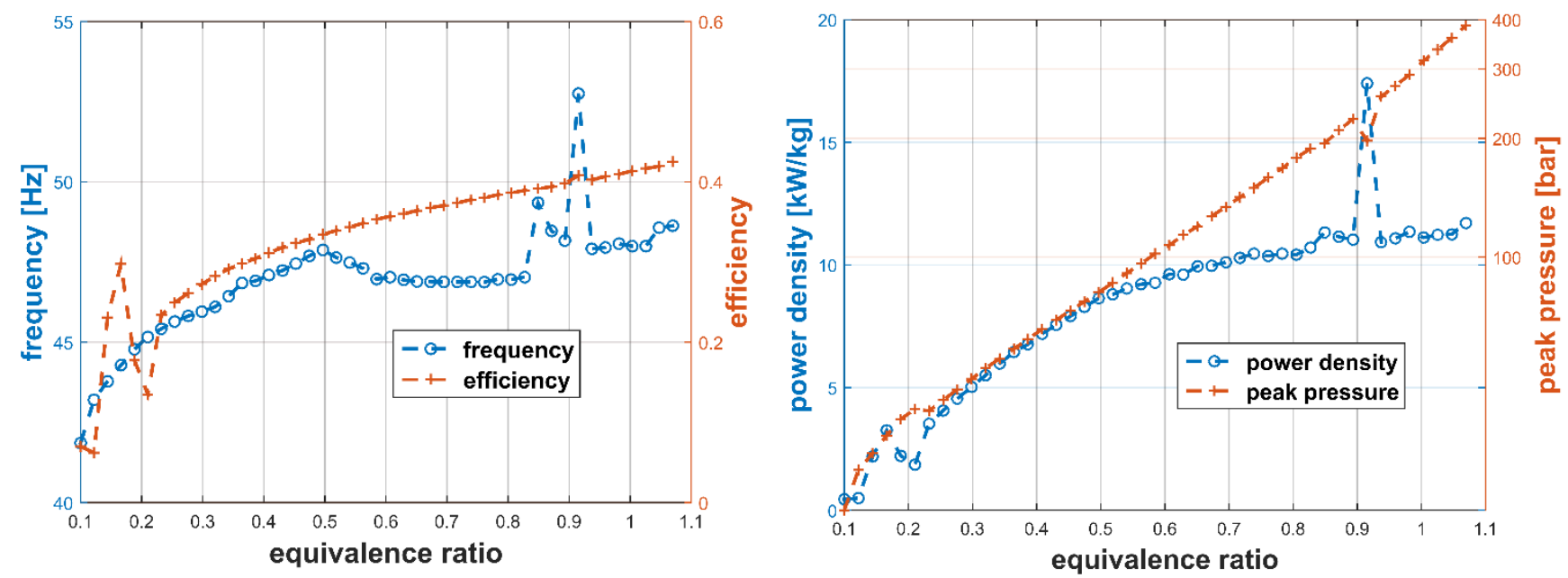

Figure 80: Parametric sweep of equivalence ratio showing its effects on frequency, efficiency, power density, and peak pressure.

Finally, the effects of EGR are shown in Figure 81 and Figure 82 . These results prove to be less insightful because the base case simulation involves the CFR ignition model (which does not include EGR effects) meaning that increase of EGR is equivalent to reduced equivalence ratio. This applies in the current model where both are related only to the amount of fuel energy added for each stroke of the engine, but not is in fact more complex within a real engine where EGR can significantly affect the incylinder processes. As it happens, the given EGR range (0-50\%) coincides with the equivalence ratio 
range of approximately $0.4-0.7$. For the same parametric sweep of EGR with the RCM ignition model, see Figure 115 and Figure 116 in Appendix C. The RCM model usage produces similar trends, except for efficiency which sees negligible increase as EGR is raised.
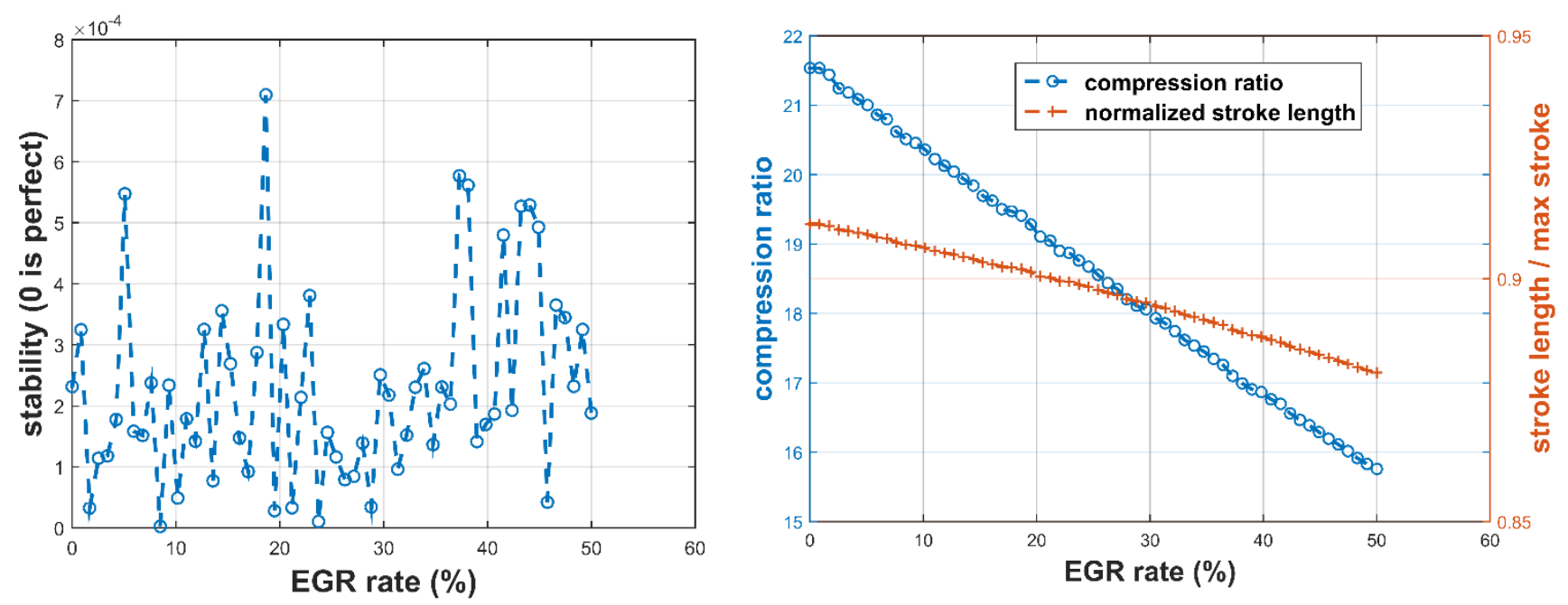

Figure 81: Parametric sweep of EGR showing its effects on stability, compression ratio, and stroke length.
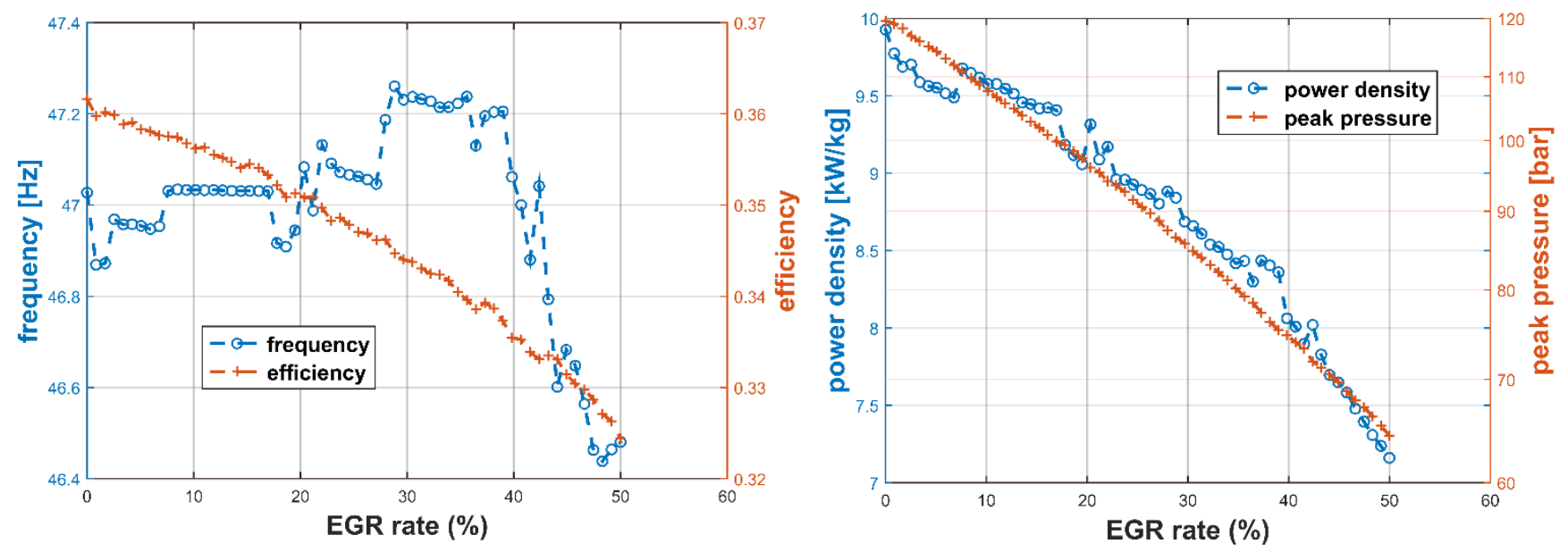

Figure 82: Parametric sweep of EGR showing its effects on frequency, efficiency, power density, and peak pressure.

Reviewing the trends shown through the previous parametric sweeps, decreasing compression ratio and stroke length are nearly always accompanied by decreases in efficiency, power density, and peak pressure. In general, it is difficult to affect frequency, but the most effective tool is shown to be the cylinder pressure (through either intake pressure or port location). Intake temperature is shown to strongly affect combustion timing and system performance. Fuel ignition too far in advance of top dead center reduces the compression ratio and stroke length leading to lower efficiency.

System stability is shown to be a function of the energy balance. Intake conditions and system geometry are seen to have lower and upper limits. These limits depend on the management of energy 
within the system. If combustion energy is not utilized or rejected, compression rises. If instead energy is used or rejected in excess to that added by the fuel, compression is reduced. Unchecked, the former will lead to excessive cylinder pressure while the latter will eventually result in system stall.

The parametric study shows that between the 'ring-up' and 'ring-down' operational boundaries is a significantly large design space around the given base case. Moreover, for a fixed design, controllable operating conditions, such as intake temperature and equivalence ratio, are shown to have significant impact on performance. Throughout this study, model simplifications limit the accuracy of the results. However, the trends demonstrated by the study are consistent and important for understanding the design space and implications for energy balance and system stability. These few plots present a small amount of information that can be gathered through parametric analysis. Supplemental figures in Appendix $\mathrm{C}$ present more data for the interested reader.

\subsection{Optimization of the SALEG}

Given the size of the design space (with eight input parameters and a wide range of possible performance targets and constraints), an optimization routine for high efficiency, steady state operation could take many forms. For this investigation, a genetic algorithm is chosen for its simplicity and robustness. The general concepts behind the GA was described in the introduction, and a flow chart is given in Figure 83 to help describe it further. 


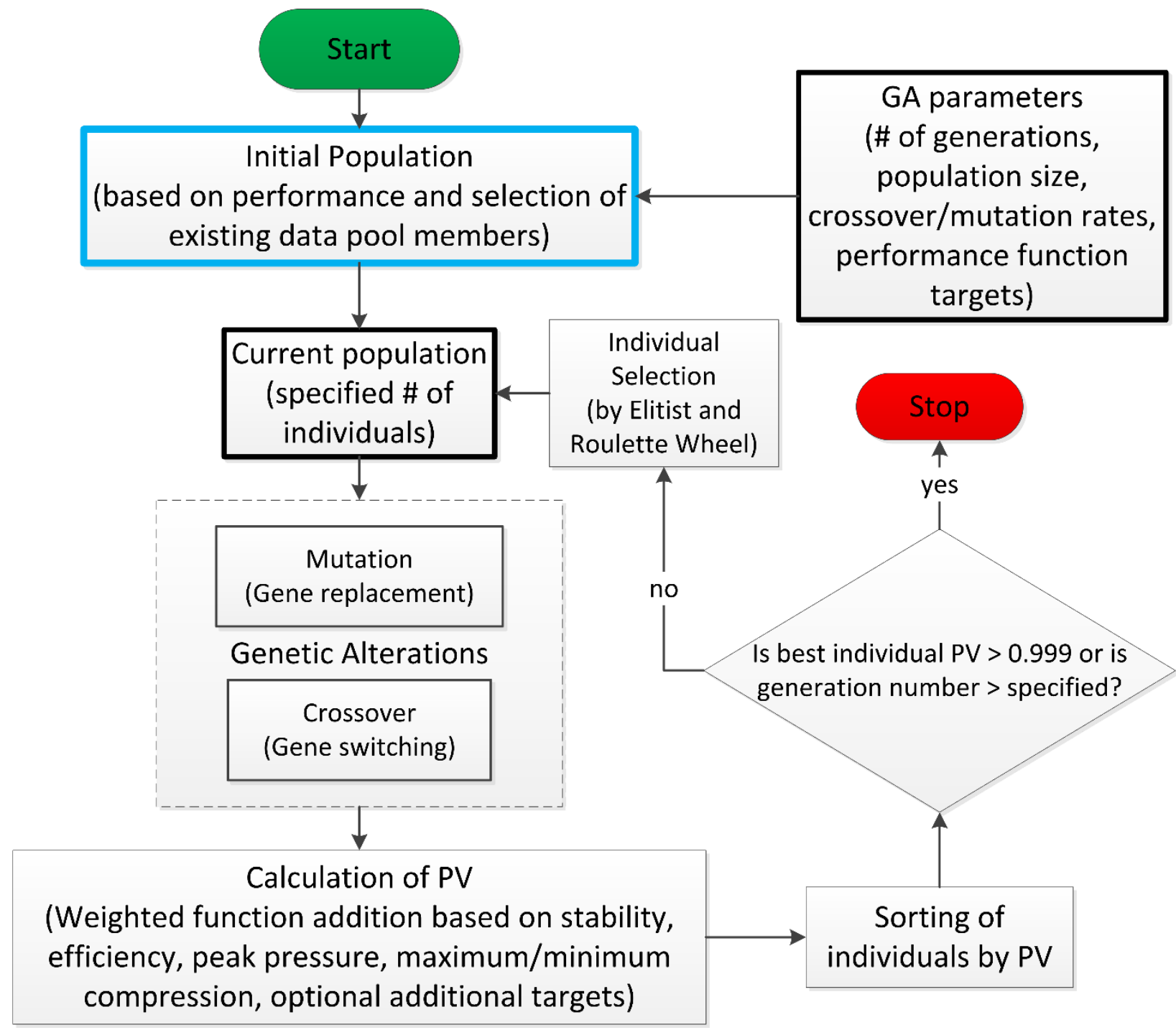

Figure 83: Flow chart diagram for the genetic algorithm optimization routine.

Essential parameters are required to begin the GA. First, the number of generations defines the maximum number of times the optimization loop is allowed to proceed. The size of the population defines the number of individuals that are held at any time. A larger population with more data might speed up the optimization process but at the cost of computational time required. Crossover and mutation rates define the percentage of individuals that undergo reproduction by genetic alternation for each generation. This may seem redundant given that the size of the population could be used to accomplish the same task. However, increasing the crossover rate relative to the mutation rate is useful for more exploratory searching of the design space while mutation is useful for exploiting the strengths of current individuals.

The current investigation is used as an example. At the start of the GA routine, a population size of 500 individuals is defined along with equal mutation and crossover rates of $25 \%$. Raising the population size or genetic alteration rates will require greater computational time for each generation, but can result 
in more robust searching of the design space. For common GA approaches, it is typical to strive for a balance between the computational requirements and GA parameters that suits that application. In addition, the application drives the suitability of the genetic alteration rates in relation to each other (mutation versus crossover). Crossover causes more significant alterations to an individual and is best suited for exploration of the design space. A 'good' crossover rate avoids local maxima within the design space while not overriding the benefits of mutation.

Meanwhile, mutation is responsible for smaller alterations to an individual and is best suited for exploitation of favorable genetic traits. A 'good' mutation rate enables improvement of existing solutions, similar to gradient-based optimization routines. For the current investigation, the given population size and alteration rates prove to be effective. Each optimization run is allowed to continue for a maximum of 70 generations or until the best individual performs better than 0.999 out a best possible of one.

When an individual undergoes mutation, each of the eight input parameters (commonly referred to as genes) may or may not be perturbed according to an additional uniformly random value. The amount of perturbation depends on a randomly selected value according to a normal distribution centered on the current value where the standard deviation is scaled according to the allowable range for the given gene. For crossover, a 'parent' individual is paired with a second, randomly selected individual to serve as its 'mate.' Then, a split point is randomly chosen based on a uniform distribution. At this split, both the parent and its mate are broken apart, and the parent's genes before the split point are combined with the mate's genes after the split point. Examples of these functions are illustrated in Figure 84.

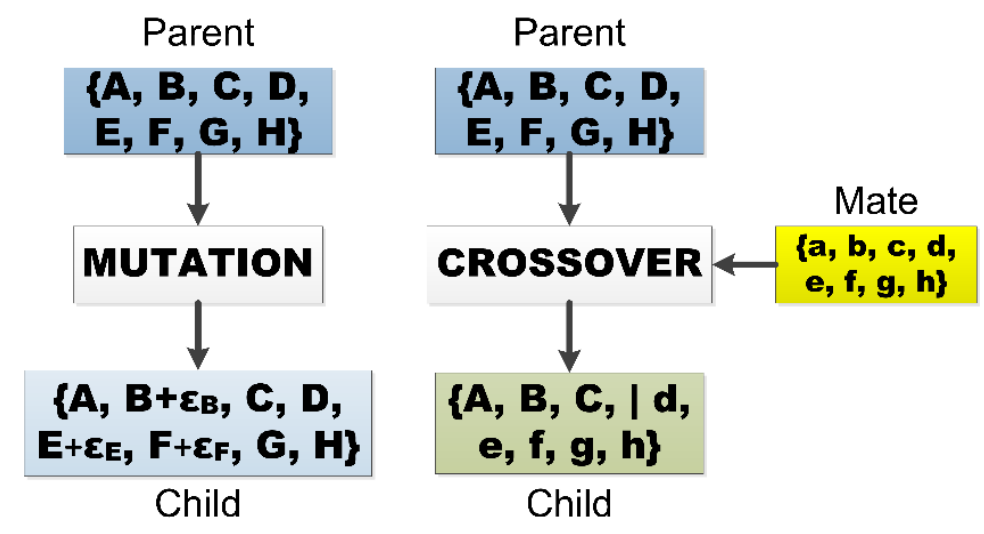

Figure 84: Illustrations to describe mutation and crossover genetic alterations.

After the new individuals have been formed, the steady state simulation routine (the same that was applied for the parametric sweep study) is applied to determine system performance with the given set of input parameters. The performance function determines a performance value (PV) for each individual. This function can be customized to accommodate multiple performance targets and system constraints. The primary optimization targets are efficiency and stability where efficiency is maximized. 
Additionally, a target power can be specified, driving the SALEG optimization towards high efficiency operation at the desired power output. Constraints are included in the performance function to help limit the optimization routine to practical responses. In the current algorithm, these constraints are applied to compression ratio and peak pressure.

With targets and constraints established, performance is assessed on a scale from zero to one where ill-performing individuals receive as low as zero and optimal individuals can receive as high as one. Weights are assigned to targets and constraints to specify the relative importance of each. A weight function is defined for each target and constraint. Exponential functions are used for the weight profiles to enable continuous profiles and encourage gradient climbing behavior within the optimization routine. The shapes of each weight function can be used to raise or lower the flexibility of a target or constraint based on a specified tolerance. For a given individual, its performance value is calculated by summing its score from each of the weighted target and constraint maps and normalizing the score against the maximum possible. Figure 85 shows the weight functions defined for determination of individual performance values. Note that variations of the profiles are explored later in this section.
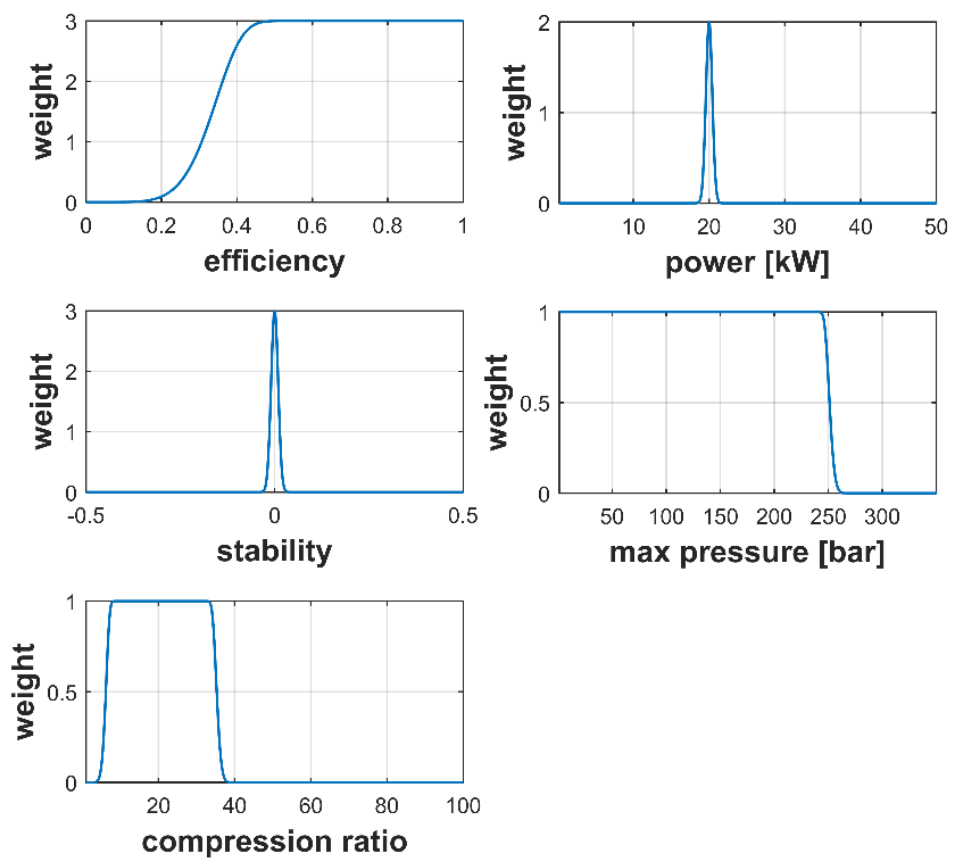

Figure 85: Typical weight function profiles for performance value calculation in the genetic algorithm optimization routine.

It is shown that stability and efficiency are weighted highest among the targets with target power following. Compression ratio and peak pressure constraints are weighted to be least impactful. The maximum pressure (peak in-cylinder pressure during steady state cycling) constraint is set at 250 bar. Though, cylinder gas blow-by past the piston rings is neglected in the simulation and will likely increase with maximum pressure. The compression ratio constraint is set to a range of $5-35$, extending above 
and below practical engine operation. These constraints can be modified, and additional constraints can be added to the performance function.

The relative weights of each target and constraint can be adjusted to affect the behavior of the genetic algorithm. The weights shown in Figure 85 reflect that high efficiency and stability are sought more than any other target or constraint. After performance is assessed for the new individuals, the original population and new individuals are combined and sorted by rank so that the best performing individual is ranked first. Selection of the individuals within the population that survive to the next generation is based on elitist and roulette wheel selection strategies. Elitist selection ensures that the best performing individual always survives to the next generation to maintain the traits of the individual. Roulette wheel selection gives more opportunity for survival to individuals with higher performance values so that ill-performing individuals could survive but are less likely to do so. The selection strategy tends to raise average performance of the population as the generations pass.
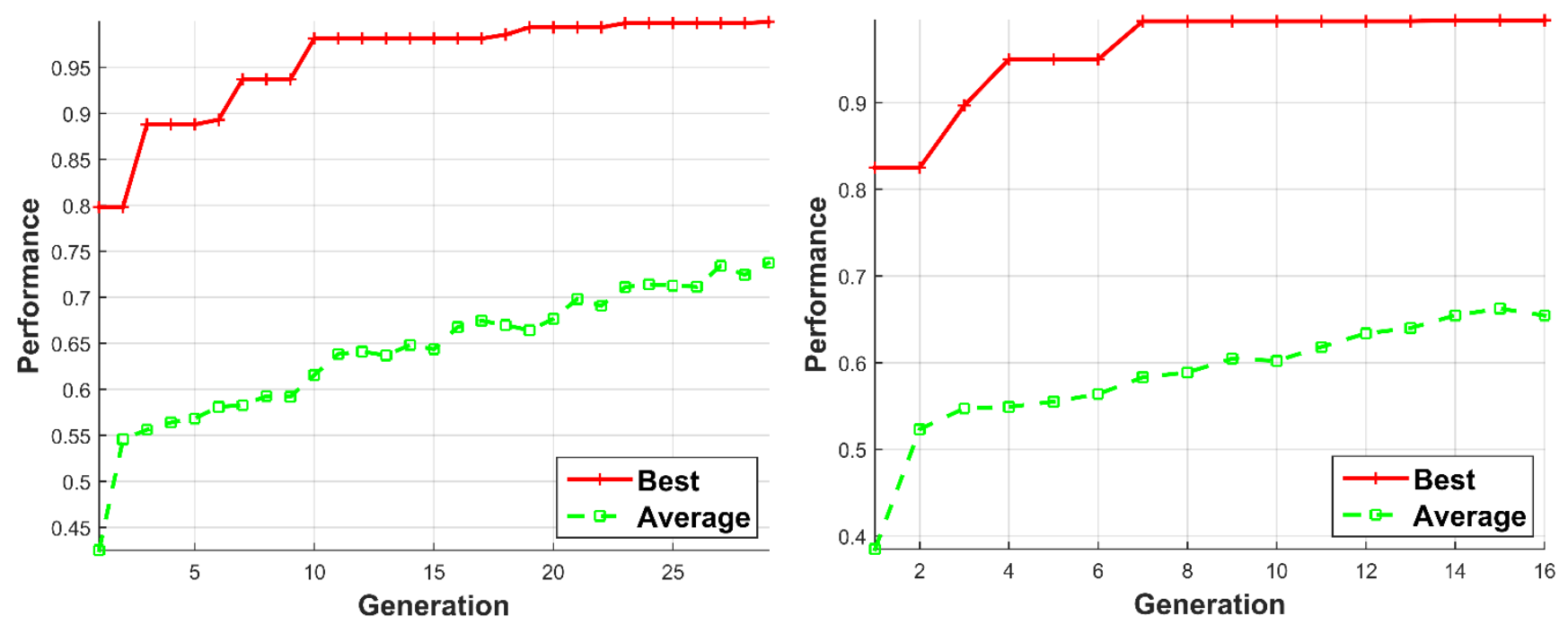

Figure 86: GA optimization response for $\mathbf{2}$ kW (left) and $\mathbf{4}$ kW (right) SALEG devices.

Given the GA as described, typical responses for the GA optimization routine with specified target power are shown in Figure 86. The average performance of the population is assessed at the end of each generation along with the best performing individual within the population. The left plot shows the optimization of a $2 \mathrm{~kW}$ SALEG while the right plot shows the same for a $4 \mathrm{~kW}$ device. In Table 9, optimized parameter sets for a range of devices is given, each based on the weight maps shown in Figure 85 but with different target powers. 
Table 9: Summary of optimized SALEG devices based on weight maps in Figure 85.

\begin{tabular}{|c|c|c|c|c|c|c|c|c|c|}
\hline Target power & $1 \mathrm{~kW}$ & $2 \mathrm{~kW}$ & $3 \mathrm{~kW}$ & $4 \mathrm{~kW}$ & $5 \mathrm{~kW}$ & $10 \mathrm{~kW}$ & $15 \mathrm{~kW}$ & $20 \mathrm{~kW}$ & $25 \mathrm{~kW}$ \\
\hline Compression ratio & 14.59 & 29.84 & 17.04 & 19.70 & 20.14 & 22.29 & 29.57 & 26.43 & 25.41 \\
\hline Frequency [Hz] & 47.49 & 49.10 & 44.14 & 73.86 & 45.55 & 48.84 & 45.97 & 44.58 & 52.51 \\
\hline Efficiency [\%] & 45.11 & 49.26 & 43.91 & 47.01 & 45.40 & 46.93 & 48.17 & 46.04 & 44.85 \\
\hline Power [kW] & 1.058 & 2.015 & 2.964 & 3.925 & 5.003 & 10.02 & 15.13 & 20.03 & 25.14 \\
\hline Peak pressure [bar] & 81.99 & 237.0 & 137.3 & 74.69 & 128.3 & 100.5 & 182.1 & 185.9 & 206.6 \\
\hline Stroke length [mm] & 50.92 & 42.26 & 75.39 & 49.26 & 84.57 & 107.9 & 125.9 & 153.6 & 148.2 \\
\hline Moving mass [kg] & 3.119 & 4.785 & 5.539 & 1.186 & 5.028 & 3.675 & 4.274 & 5.769 & 6.463 \\
\hline Power density [kW/kg] & 0.3392 & 0.4211 & 0.5351 & 3.3094 & 0.9950 & 2.727 & 3.540 & 3.472 & 3.890 \\
\hline Bore $[\mathrm{mm}]$ & 25.34 & 24.34 & 25.03 & 47.65 & 34.47 & 54.48 & 47.56 & 49.87 & 60.00 \\
\hline $\begin{array}{c}\text { Maximum stroke } \\
\text { to bore }\end{array}$ & 2.305 & 1.857 & 3.387 & 1.144 & 2.710 & 2.167 & 2.833 & 3.323 & 2.672 \\
\hline $\begin{array}{l}\text { Neutral clearance } \\
\text { length [mm] }\end{array}$ & 29.21 & 22.60 & 42.39 & 27.26 & 46.70 & 59.02 & 67.37 & 82.85 & 80.16 \\
\hline $\begin{array}{c}\text { Actual } \\
\text { displacement [cc] }\end{array}$ & 51.36 & 39.33 & 74.19 & 175.7 & 157.8 & 503.1 & 447.3 & 600.1 & 838.1 \\
\hline Port location [\%] & 27.87 & 47.41 & 43.47 & 39.83 & 40.40 & 45.24 & 43.50 & 44.45 & 38.92 \\
\hline Intake pressure [bar] & 1.426 & 1.695 & 1.715 & 1.420 & 1.315 & 1.389 & 1.168 & 1.489 & 2.355 \\
\hline Intake temperature [K] & 250.0 & 250.0 & 250.0 & 319.3 & 263.5 & 250.0 & 250.0 & 250.0 & 259.2 \\
\hline $\begin{array}{c}\text { Spring } \\
\text { stiffness [kN/m] }\end{array}$ & 100.0 & 147.1 & 178.0 & 15.00 & 151.7 & 89.87 & 96.67 & 144.8 & 202.2 \\
\hline Equivalence ratio & 0.2264 & 0.8661 & 0.6170 & 0.2418 & 0.6550 & 0.3000 & 0.6286 & 0.5682 & 0.2426 \\
\hline EGR rate [\%] & 10.60 & 34.02 & 25.51 & 7.718 & 30.73 & 18.57 & 8.995 & 20.81 & 22.32 \\
\hline
\end{tabular}

Examining the data, very few trends are evident. Generally, bore and neutral clearance length (maximum stroke) rise with power output (corresponding to longer stroke and greater displacement). The strong weighting for efficiency helps to ensure that the $>40 \%$ target is met. Intake temperature is consistently low. Referring back to the parametric sweep, lower intake temperature enables higher cylinder energy, higher compression ratio, and greater efficiency. The $4 \mathrm{~kW}$ SALEG device stands out amongst the other optimizations with substantially higher frequency. The same device also records the smallest maximum stroke to bore ratio, smallest spring stiffness, greatest power density, and lowest peak cylinder pressure. The relatively high power density is related to the high frequency (which enables smaller alternator design) and low spring stiffness (recalling the total moving mass relationship with spring stiffness discussed previously). The relatively high frequency is also facilitated by the lower maximum stroke to bore ratio (demonstrated by the left-hand plot of Figure 118). 
The wide range of results points towards a flaw in the optimization routine. The GA did find parameter sets which met the performance requirements - the performance value for each individual in Table 9 is greater than 0.998 out of a maximum of one. However, the combination of targets and constraints given in Figure 85 allows multiple high performance solutions to exist. To address this fault, the compression ratio constraint is tightened to an upper limit of 25 . These results are summarized in Table 10.

Table 10: Summary of optimized SALEG devices with varied target power and tightened compression ratio constraint rangind from 5-25.

\begin{tabular}{|c|c|c|c|c|c|c|c|c|c|}
\hline Target power & $1 \mathrm{~kW}$ & $2 \mathrm{~kW}$ & $3 \mathbf{k W}$ & $4 \mathrm{~kW}$ & $5 \mathrm{~kW}$ & $10 \mathrm{~kW}$ & $15 \mathrm{~kW}$ & $20 \mathrm{~kW}$ & $25 \mathrm{~kW}$ \\
\hline Compression ratio & 18.02 & 20.15 & 23.07 & 22.53 & 21.59 & 23.48 & 24.13 & 23.50 & 21.96 \\
\hline Frequency [Hz] & 57.62 & 65.33 & 59.56 & 64.09 & 56.07 & 52.51 & 56.42 & 56.55 & 51.86 \\
\hline Efficiency [\%] & 47.71 & 48.9 & 48.46 & 47.68 & 45.17 & 42.62 & 42.56 & 41.81 & 39.34 \\
\hline Power [kW] & 0.9833 & 2.000 & 2.975 & 4.025 & 4.996 & 9.994 & 15.01 & 20.04 & 25.04 \\
\hline Peak pressure [bar] & 54.50 & 86.82 & 155.6 & 80.60 & 129.8 & 101.9 & 166.2 & 104.1 & 76.28 \\
\hline Stroke length [mm] & 32.50 & 38.50 & 47.5 & 52.56 & 67.44 & 96.51 & 110.9 & 131.4 & 164.4 \\
\hline Moving mass [kg] & 2.172 & 1.755 & 3.161 & 2.393 & 3.747 & 4.428 & 4.326 & 2.717 & 5.077 \\
\hline Power density [kW/kg] & 0.4530 & 1.140 & 0.9412 & 1.682 & 1.333 & 2.257 & 3.470 & 7.376 & 4.932 \\
\hline Bore $[\mathrm{mm}]$ & 37.50 & 37.50 & 36.89 & 54.18 & 46.13 & 60.00 & 61.61 & 77.85 & 97.50 \\
\hline $\begin{array}{l}\text { Maximum stroke } \\
\text { to bore }\end{array}$ & 0.9685 & 1.134 & 1.404 & 1.060 & 1.604 & 1.752 & 1.955 & 1.837 & 1.847 \\
\hline $\begin{array}{c}\text { Neutral clearance } \\
\text { length [mm] }\end{array}$ & 18.16 & 21.26 & 25.90 & 28.72 & 37.00 & 52.55 & 60.22 & 71.52 & 90.05 \\
\hline $\begin{array}{c}\text { Actual } \\
\text { displacement [cc] }\end{array}$ & 71.79 & 85.04 & 101.5 & 242.4 & 225.4 & 545.8 & 661.2 & 1251 & 2455 \\
\hline Port location [\%] & 29.72 & 29.07 & 28.50 & 19.52 & 19.99 & 40.03 & 15.60 & 33.83 & 26.50 \\
\hline Intake pressure [bar] & 0.9000 & 1.274 & 1.774 & 0.9000 & 1.388 & 1.250 & 1.473 & 1.332 & 0.9000 \\
\hline Intake temperature [K] & 250.0 & 250.0 & 250.0 & 250.0 & 250.0 & 250.0 & 250.0 & 250.0 & 250.0 \\
\hline $\begin{array}{c}\text { Spring } \\
\text { stiffness }[k N / m]\end{array}$ & 63.28 & 43.47 & 86.91 & 53.17 & 101.3 & 132.0 & 96.01 & 29.87 & 121.3 \\
\hline Equivalence ratio & 0.2000 & 0.2065 & 0.2000 & 0.2000 & 0.2000 & 0.2784 & 0.2000 & 0.2000 & 0.2000 \\
\hline EGR rate [\%] & 20.89 & 11.39 & 3.64 & 22.62 & 9.818 & 12.34 & 17.75 & 16.59 & 19.13 \\
\hline
\end{tabular}

Comparing these results with the previous set where compression was constrained from 5-35 (Table 9), it is evident that limiting the compression ratio has significant impact on the design optimization. Reducing the compression ratio constrains the optimization space to reduce maximum stroke to bore ratio. This in turn raises the frequency, reduces moving mass, and increases power density (as compared to the higher compression ratio results). 
The downfalls of this design regime are low equivalence ratio, low intake temperature, and for some designs, low intake pressure. While auto-ignition of fuel may be possible for these ultra-lean conditions, actual engine operation will be greatly affected by charge stratification. The risk of misfire increases as equivalence ratio is reduced. Additionally, intake temperature and intake pressure are not reflective of common ambient conditions.

Focusing on the design of the $4 \mathrm{~kW}$ device, a range of optimization points can be achieved. For these designs, the lower limit for intake temperature is $300 \mathrm{~K}$, the lower limit for intake pressure is $1 \mathrm{bar}$, and the lower limit for equivalence ratio is 0.35 . Having retained the data collected through many optimization runs, multi-dimensional scatter plots can be used visually explore the design space for the described case. The first plot is given in Figure 87.

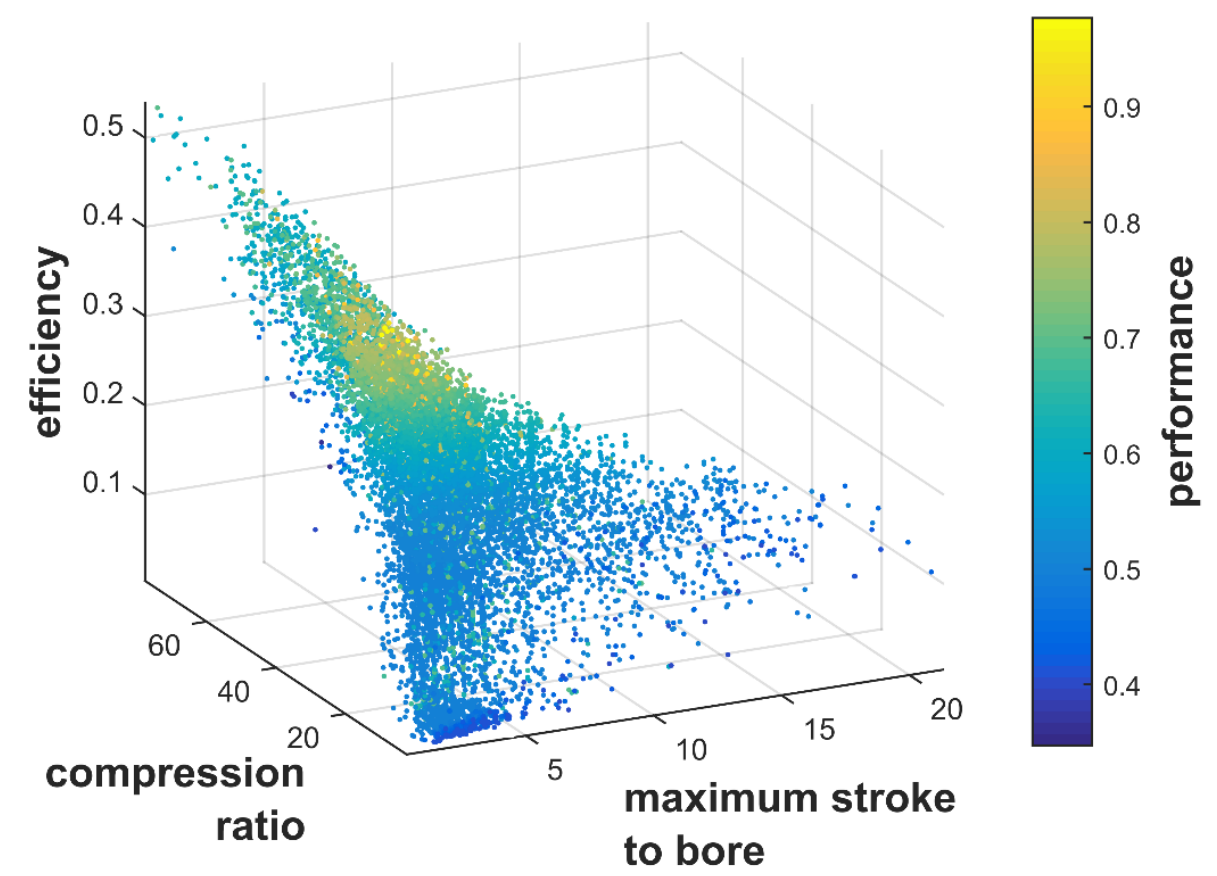

Figure 87: Design map of maximum stroke to bore ratio, compression ratio, efficiency, and overall performance for the $4 \mathrm{~kW}$ SALEG. Lower limits are applied so that intake temperature is greater than $300 \mathrm{~K}$, intake pressure is greater than 1 bar, and equivalence ratio is greater than 0.35 for all data points.

For each of the data points shown in Figure 87, the performance value is calculated according to the maps given in Figure 85 except that the compression ratio constraint is set for 5-25. The design map shows that peak performance is achieved with low maximum stroke to bore ratio, maximum compression ratio (with respect to the upper constrain limit), and results in an efficiency grouping around $40 \%$. The plot demonstrates only a single design zone. The next design map relates the maximum stroke to bore ratio to frequency and power density (Figure 88). 


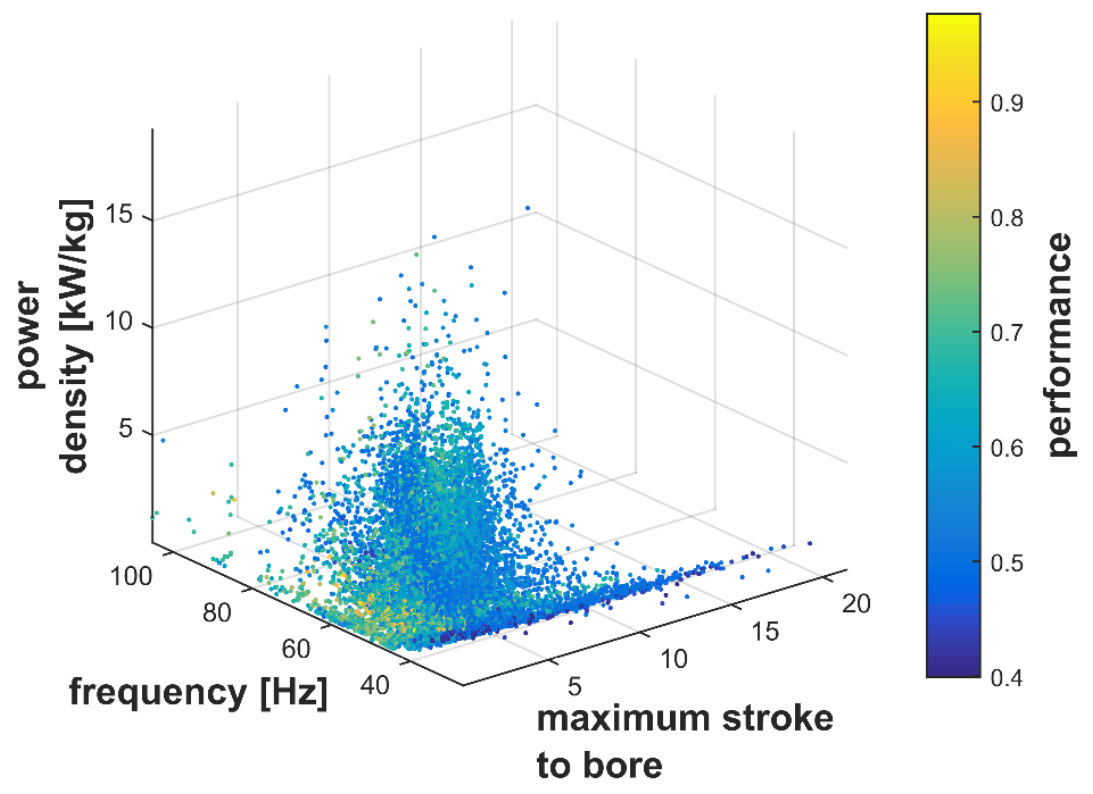

Figure 88: Design map of maximum stroke to bore ratio, frequency, power density, and overall performance for the 4 kW SALEG. Lower limits are applied so that intake temperature is greater than $300 \mathrm{~K}$, intake pressure is greater than 1 bar, and equivalence ratio is greater than 0.35 for all data points.

This second scatter plot with maximum stroke to bore ratio shows a bifurcation in the design space. While the performance is maximized for maximum stroke to bore ratio values near 1 with frequency grouped around $60 \mathrm{~Hz}$, a second design space with ratios around 5 shows higher power density. It is worth recalling that power density is not included in the current performance definition. The next plots shift the focus to intake temperature and equivalence ratio.

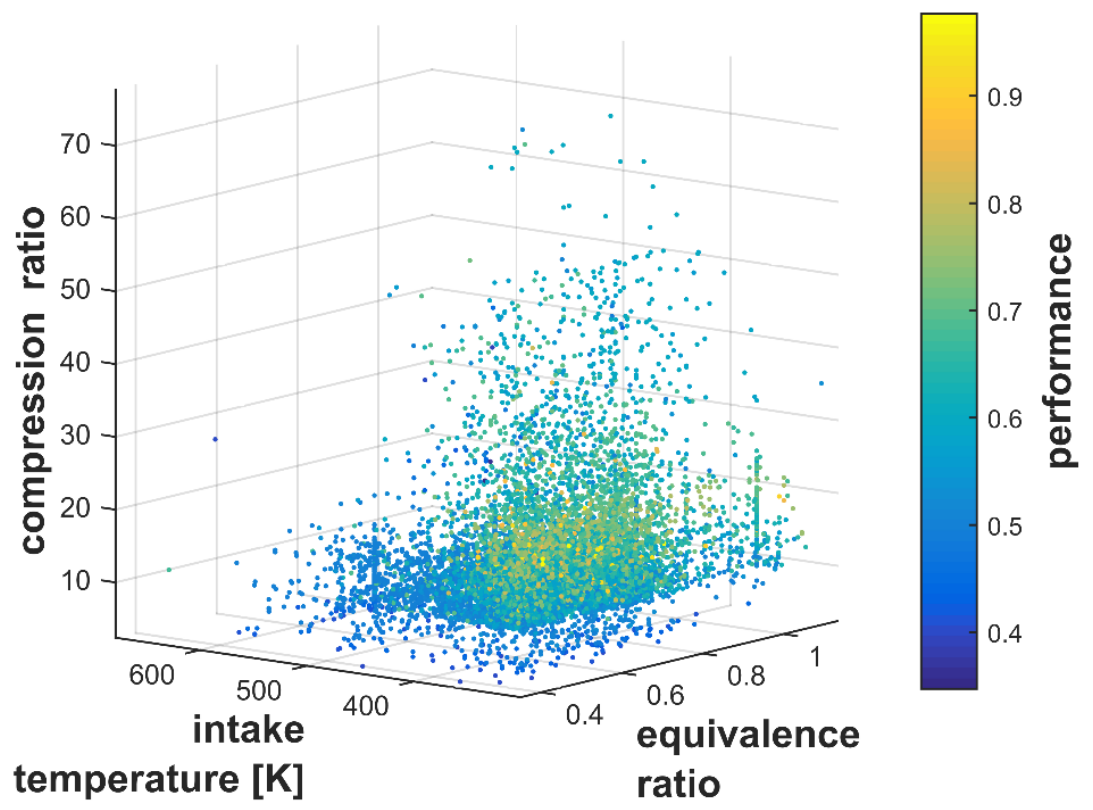

Figure 89: Design map of equivalence ratio, intake temperature, compression ratio, and overall performance for the 4 kW SALEG. Lower limits are applied so that intake temperature is greater than $300 \mathrm{~K}$, intake pressure is greater than 1 bar, and equivalence ratio is greater than 0.35 for all data points. 
Figure 89 shows the effects of equivalence ratio and intake temperature on compression ratio and design performance. Referring to the compression ratio, a 'floor' is observed representing the threshold for combustion and SALEG stability. Also, the high performance individuals are grouped around compression ratio of 20 . As intake temperature is reduced, the compression ratio ceiling is raised allowing for more high performance individuals. This concurs with the findings seen throughout the optimization and parametric studies. For equivalence ratio, high performance individuals are seen throughout the domain of equivalence ratio values available in the data set, but are grouped between the lower limit of 0.35 and about 0.7 . This indicates that optimized performance can be found for a wide range of equivalence ratio. Improvement of the cylinder model, specifically regarding the combustion process, could very likely affect the design space around equivalence ratio.

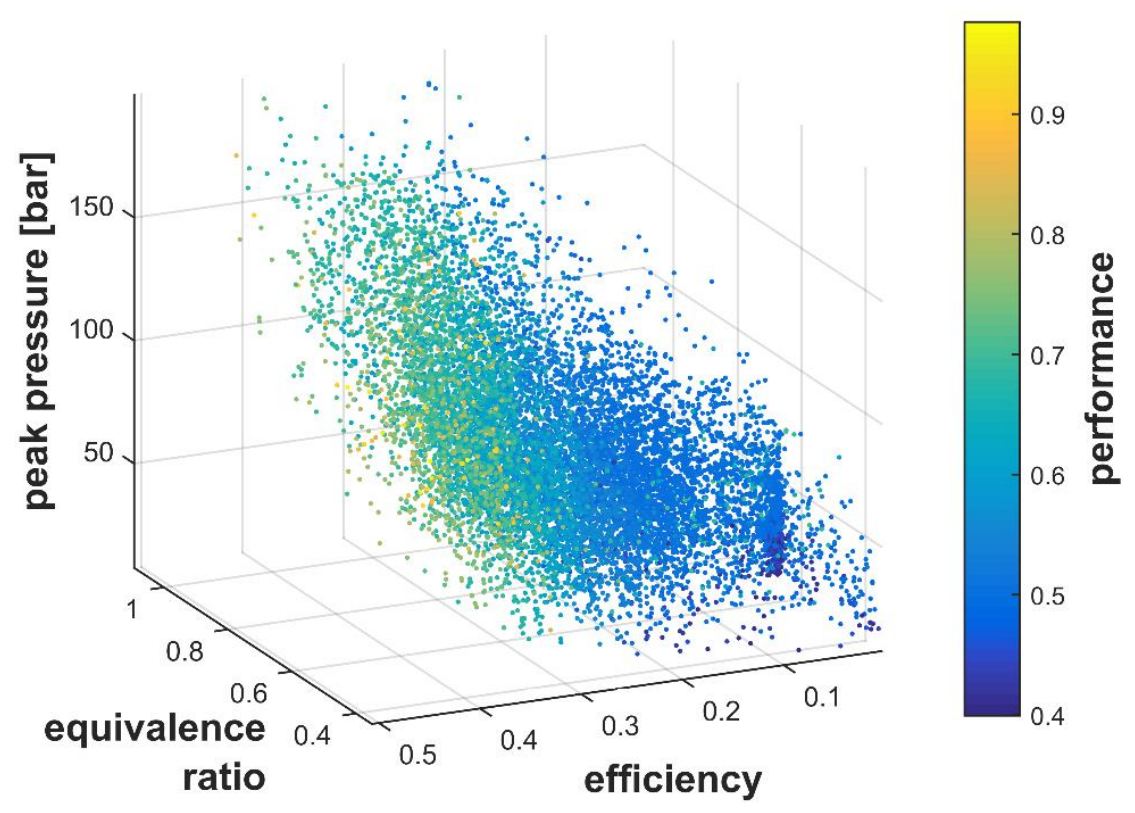

Figure 90: Design map of equivalence ratio, efficiency, peak cylinder pressure, and overall performance for the $4 \mathrm{~kW}$ SALEG. Lower limits are applied so that intake temperature is greater than $300 \mathrm{~K}$, intake pressure is greater than 1 bar, and equivalence ratio is greater than 0.35 for all data points.

Figure 90 shows a second perspective of the equivalence ratio design space with respect to peak pressure, efficiency, and performance. Efficiency and performance are strongly related due to the definition of performance. In the high performance region, corresponding to efficiencies around $40 \%$, wide ranges for both equivalence ratio and peak pressure are seen. Lastly, the design space for intake temperature, frequency, power, and performance is shown in Figure 91. 


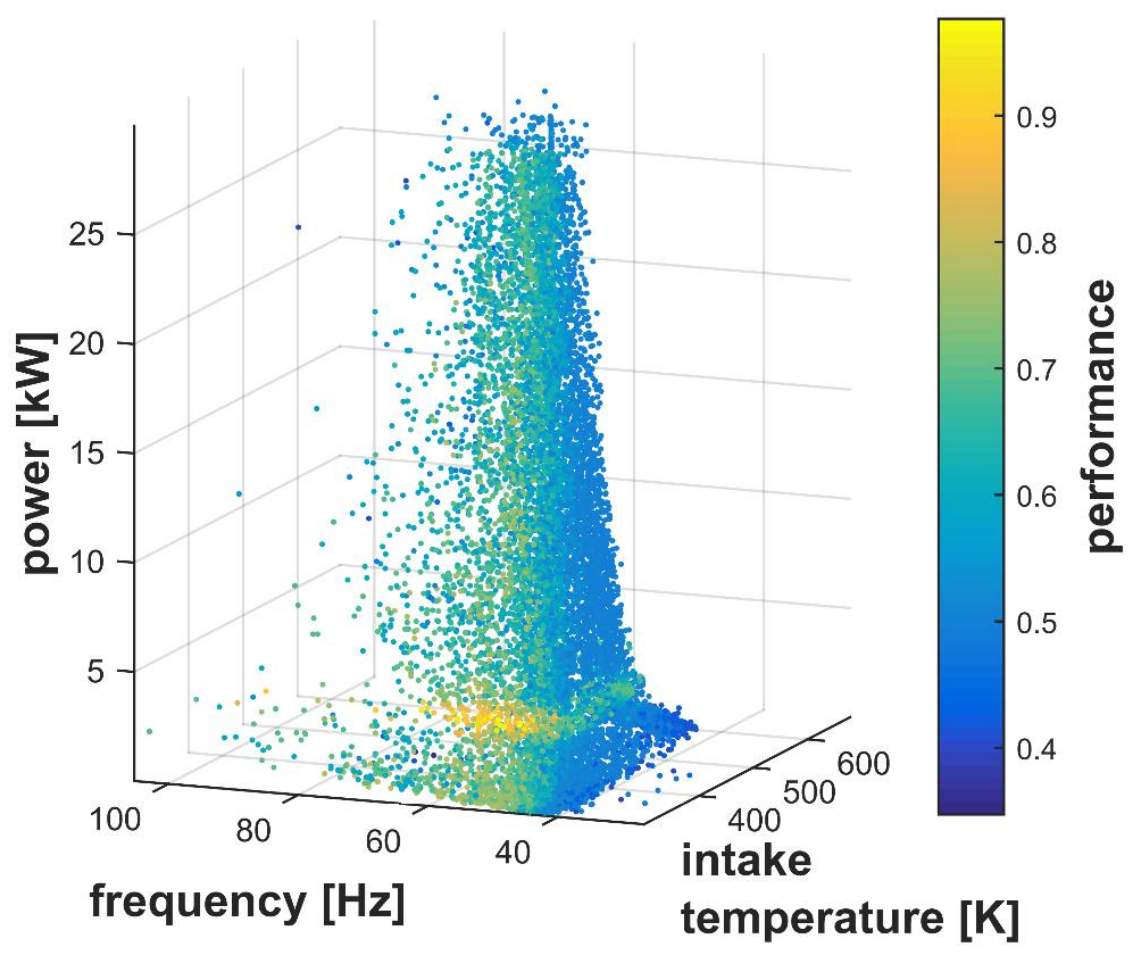

Figure 91: Design map of intake temperature, frequency, power, and overall performance for the 4 kW SALEG. Lower limits are applied so that intake temperature is greater than $300 \mathrm{~K}$, intake pressure is greater than 1 bar, and equivalence ratio is greater than 0.35 for all data points.

Figure 91 shows a well-defined design space for the power output as a function of engine speed and intake temperature. A band of comparatively high performance cases is seen along the $4 \mathrm{~kW}$ plane. Otherwise, the design space for intake temperature and frequency narrows as power demand is raised. Again, lower intake temperature results in higher performance. Performance is not significantly affected by frequency so long as frequency remains above a lower threshold (about $45 \mathrm{~Hz}$ for the current space).

The design space scatter plots serve as visual aids for understanding the design space of the simulated SALEG. They also serve to verify the repeatability of the optimization routine. The design space plots (specifically Figure 87 and Figure 91) show unambiguous regions of optimal design.

Despite the repeatability of the design optimization, it is worth examining the weights given for the targets and constraints to understand their effect on the design space. Thus, Table 11 is given to show the effects of four different weighting schemes. Each set of weights is given as a set of integers ordered according to the following: \{efficiency, stability, power, compression ratio, peak pressure\}. For each set of weights, the top two optimization points are provided. Examining the table, the top two design points are identical regardless of the weighting. This is in part due to the fact that the same source of data was used to quickly search the design space. However, the data source consists of many thousands of design points, so is reasonable for this sensitivity analysis. 
Table 11: Sensitivity of $4 \mathrm{~kW}$ optimized designs to variations of the GA performance weights.

\begin{tabular}{|c|c|c|c|c|c|c|c|c|}
\hline \multirow{2}{*}{$\frac{\text { Weights* }^{*}}{\text { Compression ratio }}$} & \multicolumn{2}{|c|}{$3,3,2,1,1$} & \multicolumn{2}{|c|}{$1,1,1,1,1$} & \multicolumn{2}{|c|}{$2,2,2,1,1$} & \multicolumn{2}{|c|}{$1,1,1,3,3$} \\
\hline & 19.64 & 21.29 & 21.29 & 19.64 & 21.29 & 19.64 & 21.29 & 19.64 \\
\hline Frequency [Hz] & 49.26 & 46.25 & 46.25 & 49.26 & 46.25 & 49.26 & 46.25 & 49.26 \\
\hline Efficiency [\%] & 42.58 & 41.77 & 41.77 & 42.58 & 41.77 & 42.58 & 41.77 & 42.58 \\
\hline Power [kW] & 3.882 & 3.953 & 3.953 & 3.882 & 3.953 & 3.882 & 3.953 & 3.882 \\
\hline Peak pressure [bar] & 96.79 & 112.2 & 112.2 & 96.79 & 112.2 & 96.79 & 112.2 & 96.79 \\
\hline Stroke length [mm] & 70.94 & 71.50 & 71.50 & 70.94 & 71.50 & 70.94 & 71.50 & 70.94 \\
\hline Moving mass [kg] & 3.871 & 8.176 & 8.176 & 3.871 & 8.176 & 3.871 & 8.176 & 3.871 \\
\hline Power density [kW/kg] & 1.0028 & 0.4835 & 0.4835 & 1.0028 & 0.4835 & 1.0028 & 0.4835 & 1.0028 \\
\hline Bore [mm] & 40.68 & 40.68 & 40.68 & 40.68 & 40.68 & 40.68 & 40.68 & 40.68 \\
\hline $\begin{array}{l}\text { Maximum stroke } \\
\text { to bore }\end{array}$ & 1.931 & 1.931 & 1.931 & 1.931 & 1.931 & 1.931 & 1.931 & 1.931 \\
\hline $\begin{array}{l}\text { Neutral clearance } \\
\text { length }[\mathrm{mm}]\end{array}$ & 39.27 & 39.27 & 39.27 & 39.27 & 39.27 & 39.27 & 39.27 & 39.27 \\
\hline $\begin{array}{c}\text { Actual } \\
\text { displacement [cc] }\end{array}$ & 184.4 & 185.9 & 185.9 & 184.4 & 185.9 & 184.4 & 185.9 & 184.4 \\
\hline Port location [\%] & 47.17 & 47.17 & 47.17 & 47.17 & 47.17 & 47.17 & 47.17 & 47.17 \\
\hline Intake pressure [bar] & 1.498 & 1.498 & 1.498 & 1.498 & 1.498 & 1.498 & 1.498 & 1.498 \\
\hline Intake temperature [K] & 305.4 & 305.4 & 305.4 & 305.4 & 305.4 & 305.4 & 305.4 & 305.4 \\
\hline $\begin{array}{c}\text { Spring } \\
\text { stiffness [kN/m] }\end{array}$ & 113.1 & 261.9 & 261.9 & 113.1 & 261.9 & 113.1 & 261.9 & 113.1 \\
\hline Equivalence ratio & 0.4190 & 0.4830 & 0.4831 & 0.4185 & 0.4831 & 0.4185 & 0.4831 & 0.4185 \\
\hline EGR rate [\%] & 21.93 & 26.12 & 26.16 & 21.93 & 26.16 & 21.93 & 26.16 & 21.93 \\
\hline
\end{tabular}

* Weights are given as ordered integer sets according to the following: \{efficiency, stability, power, compression ratio, peak pressure $\}$. The weighting given in Figure 85 would be represented by $\{3,3,2,1,1\}$.

To finish the optimization study, a final design point is presented in Table 12. For this design point, the performance function did not include a target power, leaving the GA open to optimize any output power within the space. The SALEG device specified in Table 12 is presented to the reader as a reasonable design solution. Given the limitations of the numeric modeling brought on by the previously described simplifications, it is recommended that the design be investigated through more sophisticated modeling of its combustion and gas exchange processes and prototype development with the intent to match the target performance. In addition, designs shown in previous tables may also be viable and might warrant similar treatment. 
Table 12: Final design optimization without target power included in performance evaluation.

\begin{tabular}{|c|c|}
\hline Compression ratio & 21.51 \\
\hline Frequency [Hz] & 52.53 \\
\hline Efficiency [\%] & 44.77 \\
\hline Power [kW] & 5.723 \\
\hline Peak pressure [bar] & 116.6 \\
\hline Stroke length [mm] & 77.09 \\
\hline Moving mass [kg] & 2.758 \\
\hline Power density [kW/kg] & 2.075 \\
\hline Bore [mm] & 40.67 \\
\hline Maximum stroke to bore & 2.080 \\
\hline Neutral clearance length [mm] & 42.30 \\
\hline Actual displacement [cc] & 200.3 \\
\hline Port location [\%] & 31.35 \\
\hline Intake pressure [bar] & 1.028 \\
\hline Intake temperature [K] & 301.3 \\
\hline Spring stiffiness [kN/m] & 75.0 \\
\hline Equivalence ratio & 0.6047 \\
\hline EGR rate [\%] & 20.93 \\
\hline
\end{tabular}

\subsection{Conclusions}

Combining the numeric model presented in Ch. 3 with simplified component design, the SALEG has been defined in terms of eight independent parameters. Sweeps of these parameters are used to study the SALEG with respect to performance characteristics such as stability, energy balance, efficiency, power density, and frequency. Employing the same steady state design simulation tool and a genetic algorithm optimization routine, the design space of the SALEG is explored and designs are presented for the SALEG with varied target output power.

The study shows that low intake temperature and low maximum stroke to bore ratio promotes high efficiency with high power density. The size and shape of the design space indicates that the currently simulated SALEG may be better suited for steady state power production below $10 \mathrm{~kW}$, as the design space narrows when target power is raised. Optimizing for efficiency with reduced compression ratio is shown to reduce the maximum stroke to bore ratio.

While this study of the design space is not exhaustive, at least one bifurcation is evident regarding the relationship between power density and maximum stroke to bore ratio. Other branches of the design space might be observed given different model conditions or optimization goals. Of particular interest is 
improvement of the relationships between EGR, port geometry, equivalence ratio, and combustion. The simplified 1-D pressure model does not account for the complex relationships between these parameters and performance characteristics.

Additionally, liberties were taken in this investigation to simplify the component design calculations. The use of mechanical compression springs is assumed, but many spring designs are available. Particularly interesting is the possibility of air springs (where the pressure in the bounce chamber can be tailored to specific operation and react to transient behavior). Assuming the air mass is negligible, bounce chambers would contribute no moving mass to the system, thus mitigating the negative impact on frequency and power density.

Recognizing that the simplifications taken in the modeling limit that accuracy of the results, this parametric and optimization study represents early steps towards a commercially viable product. The SALEG is shown to have excellent potential, and defining the true and ideal operating regime will likely require years of prototype testing and validation. However, optimization work similar to what has been presented here has also been instrumental in successful proposal efforts at WVU, and it will presumably similar research efforts that move the free piston linear engine towards optimized operation. 


\section{CH. 5 TRANSIENT OPERATION AND CONTROL}

One of the most significant challenges barring the path of the free piston linear engine is that of piston motion control. Without the constraints of mechanical linkages (such as the crankshaft and piston rod in the conventional engine), the free piston translator moves only according to its own inertia and the forces acting upon it. Thus, cycle-to-cycle variation becomes both a source of opportunity (enabling the potential to affect compression ratio to enhance performance) and a source of difficulty. Performance of the SALEG at steady state operation was explored through the parametric study, showing that high efficiency could be reached under the right conditions. During transient operation, however, maintaining operation becomes the primary concern while the return to high performance becomes the secondary goal. The first transient event to be discussed is that of motored, resonant start-up.

\subsection{Resonant Start-Up}

The concept of employing device resonance to achieve start-up stems from the fact that the free piston within the SALEG is essentially a mass-spring-damper system. The engine cylinders and compression springs (still assumed to be mechanical for the current investigation) provide an effective stiffness while the heat transfer and friction combine to dampen the oscillations of the translator. Without combustion, the engine cylinders operate along their motoring curves, and the alternator is inverted to provide an excitation force for the translator. The excitation continues until sufficient stroke and compression ratio are achieved to facilitate fuel injection and combustion. Once combustion is achieved, the alternator inverter is disengaged, and the alternator is allowed to draw power.

This bounced starting method has been suggested by various researchers and explored in both analytical and numerical spaces for a dual cylinder free piston device. In 2006, an investigation from Kyungnam University in South Korea considered the use of an inverted three-phase permanent magnet linear alternator as a motor to force the oscillation in a linear engine at resonance to achieve sufficient compression [104]. They concluded that feedback control from translator position sensing would be required for precise control of the alternator inverter. In 2014, researchers from Newcastle University and the Beijing Institute of Technology published the developmental and experimental results which showed the motored resonance approach can achieve sufficient compression in a dual piston free piston engine device [33]. The Beijing researchers then extended the bouncing start-up investigation in [36], where a constant starting force, which changed with velocity, was employed to achieve a pulse width modulated square wave. For a $3 \mathrm{~kg}$ translator mass with bore of $50 \mathrm{~mm}$, they simulated a compression ratio of $\sim 20$ and engine frequency of $\sim 21 \mathrm{~Hz}$ with a forcing amplitude of $55 \mathrm{~N}$. They parametrically explored the effects of cylinder bore and translating mass, concluding that decreased stroke-to-bore ratio increases steady state frequency response. Reducing the translator mass can also increase frequency but additionally 
results in lower force capabilities of the electric motor due to physical limitations. Former research at WVU suggested that, like other dynamic resonance systems, it would be frequency of the excitation force rather than its magnitude that would lead to suitable stroke length and compression ratio [82].

Recent research by the author investigated the resonant start-up of a single cylinder free piston linear engine, also a steady state generator device [7]. The work was conducted with a numeric model and found that motoring force frequency is indeed more impactful than motoring force magnitude. It was shown that friction is highly influential on the resonant behavior of the free piston, but that the effects (as well as the effects of heat transfer) were more significant with regard to the overshoot and settling time responses of the free piston rather than the steady state response. While the single cylinder device differs from the dual cylinder SALEG in dynamic profiles as well as architecture, it is expected that these findings hold true for any free piston engine. It is also expected that the resonance of any system will depend on its geometric and physical parameters.

However, unlike the former investigation, the purpose of the current research is not to explore the effects of device parameters on resonance, but rather to achieve start-up of a given device. This depends on the implementation of a controller that can detect the dynamic state of the translator and respond to affect motor operation and resulting compression ratio. Then, after sufficient compression has been achieved, the controller must cause fuel injection and engagement of the alternator. Additionally, the controller should be able to provide transitional support to ensure successful steady state operation with combustion and alternator load. To facilitate the transient study, the following SALEG device is presented with respect to its nominal steady state performance. The device is described through Figure 92, Figure 93, and Table 13.
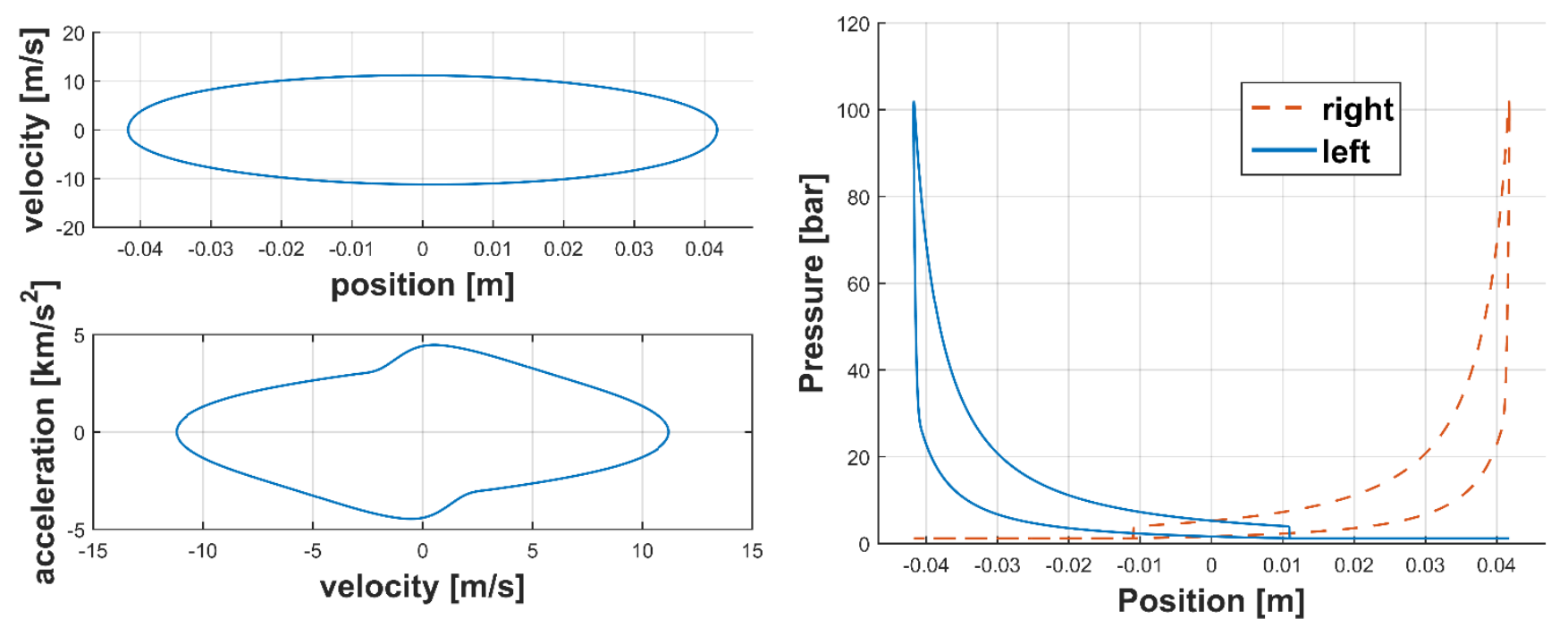

Figure 92: Translator dynamic profiles and pressure traces for the Ch. 5 base case. 

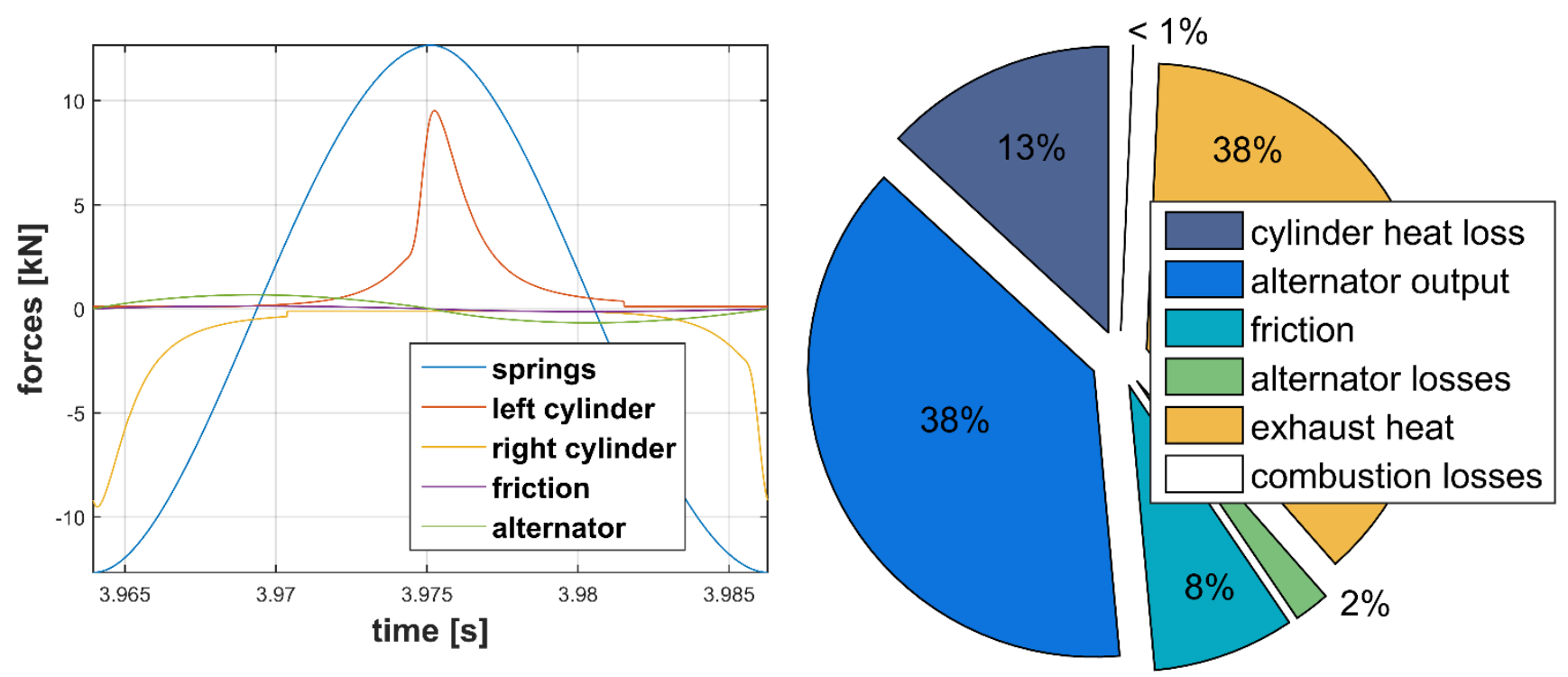

Figure 93: Forces acting on the translator and steady state energy analysis of the Ch. 5 base case.

Table 13: Summary of design parameters and stead state performance characteristics of the Ch. 5 base case.

\begin{tabular}{|r|l|}
\hline Bore & $34.5 \mathrm{~mm}$ \\
\hline Maximum stroke & $93.4 \mathrm{~mm}$ \\
\hline Port closing distance from cylinder head & $57.6 \mathrm{~mm}$ \\
\hline Equivalence ratio & 0.655 \\
\hline EGR & $30.7 \%$ \\
\hline Intake pressure & $1.20 \mathrm{bar}$ \\
\hline Intake temperature & $263^{\circ} \mathrm{K}$ \\
\hline Cylinder mixture mass & $0.0853 \mathrm{~g}$ \\
\hline Wall temperature & $550{ }^{\circ} \mathrm{K}$ \\
\hline Spring stiffness & $151.7 \mathrm{kN} / \mathrm{m}$ \\
\hline Total moving mass & $4.93 \mathrm{~kg}$ \\
\hline Stroke length & $83.5 \mathrm{~mm}$ \\
\hline Compression ratio & 17.9 \\
\hline Frequency & $44.8 \mathrm{~Hz}$ \\
\hline Power & $3.81 \mathrm{~kW}$ \\
\hline System efficiency & $38.2 \%$ \\
\hline Mean alternator force & $449 \mathrm{~N}$ \\
\hline Peak cylinder pressure & $102 \mathrm{bar}$ \\
\hline Peak translator acceleration & $4.46 \mathrm{~km} / \mathrm{s}^{2}$ \\
\hline Peak translator velocity & $11.2 \mathrm{~m} / \mathrm{s}$ \\
\hline Fuel energy added per cycle & $222 \mathrm{~J}$ \\
\hline Beading parameter, & 65 \\
\hline a fix &
\end{tabular}

Because a fixed cylinder wall temperature is used in the current investigation, this start-up discussion is limited to the hot-start scenario where the engine is at rest but is already at steady state operational temperatures. Given the base case SALEG device, the numeric model is altered to include two modes of operation for the alternator. Motoring mode is modeled with a sinusoidal force profile with 
constant frequency and amplitude while the previously used alternator mode is maintained to convert translator kinetic energy into output power. Examining the natural resonance of the device can be accomplished by applying a spectrum of motor frequency and amplitude conditions until the translator reaches steady state under the motored condition. For this phase of the study, the controller is not allowed to transition the device out of motored operation thus allowing steady state to be reached for any motor excitation condition. Figure 94 shows the effects of varied motor force amplitude on stroke length (normalized against maximum stroke) and compression ratio.

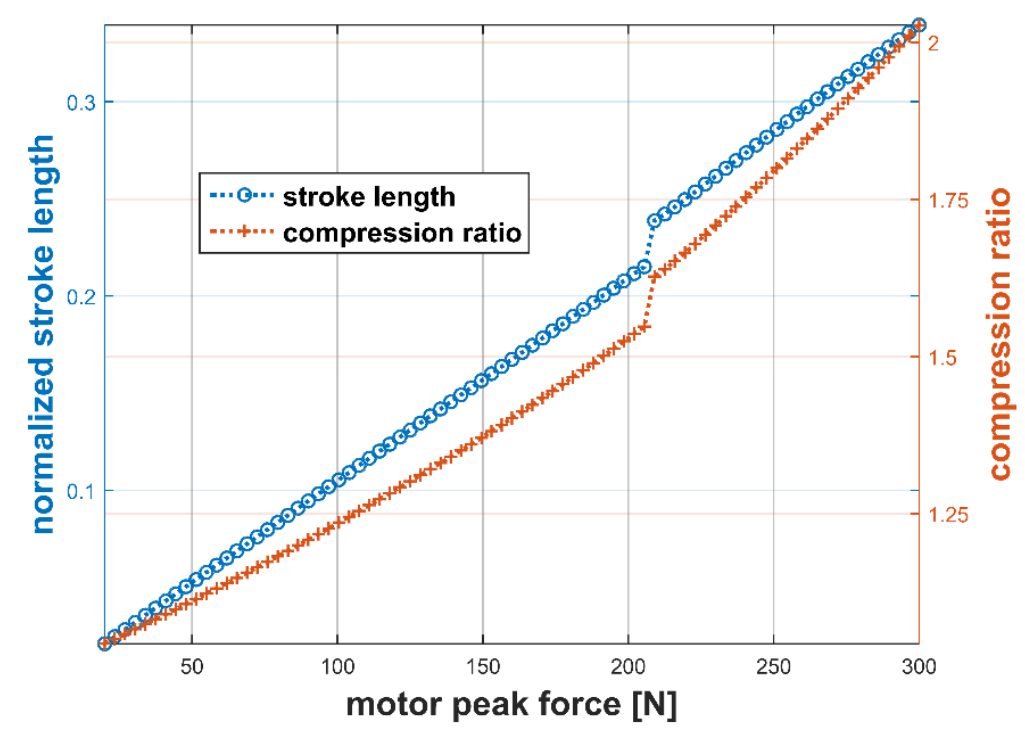

Figure 94: Simulation of base case being motored by the inverted alternator with sinusoidal force profile. Force frequency is $35 \mathrm{~Hz}$ and force amplitude is varied.

Noting the scale of the left and right vertical axes, varying the motor force amplitude brings no significant change to the steady state operation of the translator. The small step change in the otherwise linear profile is a result of the stroke having increased sufficiently to open the ports and enable gas exchange. It may be that continuing to increase the motor amplitude could eventually achieve desired compression and stroke, but the force amplitude of the motor is physically limited depending on alternator parameters. Eventually, further increase of the force amplitude would require a larger alternator. Instead, it is the frequency of the motoring force that should be varied to achieve sufficient stoke and compression. This is demonstrated in Figure 95. 


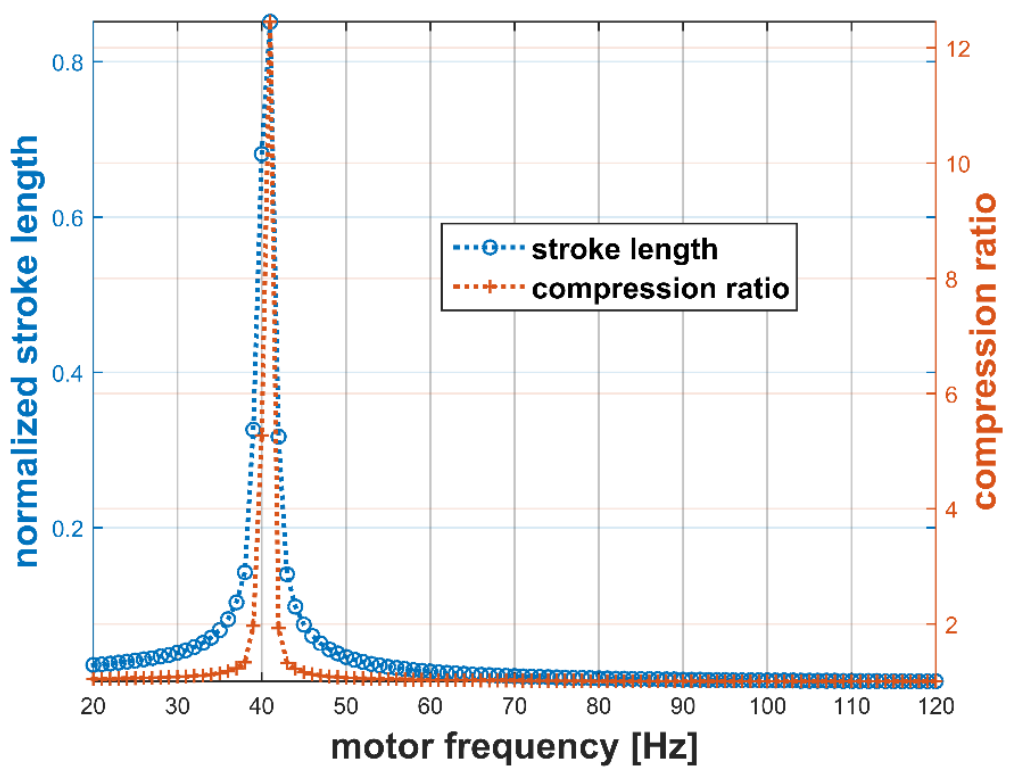

Figure 95: Simulation of base case being motored by the inverted alternator with sinusoidal force profile. Force amplitude is $66 \mathrm{~N}$ and force frequency is varied.

By varying the motor frequency, the natural resonance of the base case SALEG device is shown to be just above forty cycles per second. The start-up concept is based on the existence of this resonant point, calling for a controller that can tune the motor frequency according to the rise or fall of the compression ratio, eventually matching the motor frequency to the resonant frequency to succeed in high compression and stroke. Theoretically, the system resonant frequency for the current SALEG device is known (by Figure 95), so it would be possible to immediately match the motor frequency and achieve desired operation to enable the transition to fired operation. However, practical operation of the device would likely affect its resonance (e.g. carbon build-up on a piston, compression spring fatigue, or cylinder gas blow-by around the piston rings). Thus, it is more desirable to construct a controller that can find the resonance and affect start-up without a priori knowledge of the resonance.

In building this controller, it is considered that the only signal information available is the translator position, but that the signal information is continuous and accurate. From the model, copious amounts of data are generated (including compression ratio, stroke length, translator dynamics, and cylinder states), but it cannot be assumed that this information is entirely available to the controller. Limiting the signals available to the controller is based on realistic considerations and results in a controller algorithm that must perform additional computations.

From the position signal, velocity and acceleration are immediately available through its derivative, though it is noted that the derivative of a noisy signal increases the noise in the derived signals. When the translator velocity equals zero, a dead center position has been reached, and at every dead center position, the stroke length and compression ratio are calculated. These calculations provide 
continuous stair-stepped signals for compression ratio and stroke length which are employed by the controller to adjust the motor frequency and alternator state as necessary.

The controller is composed of four parts: a state flow diagram to command the mode of the alternator (motor or alternator); the motor controller which adjusts the motor frequency to increase compression ratio and stroke; the alternator controller that was previously discussed in section 3.6.6; and the fuel control which at this point is limited to one of two states, command fuel at a constant equivalence ratio or command no fuel. The state flow diagram operates according to a simple threshold. If the compression ratio is above the threshold, fired operation is commanded; otherwise, motored operation is commanded. For the current device, a suitable threshold is found to be a compression ratio of twelve. The fuel control needs no further explanation and details for the alternator controller can be found in the previous section.

The motor controller consists of proportional and integral components. The controller is built on the premise of beginning with a motor frequency that is known to be less than the resonant frequency. While this limits its robustness, it enables a simpler controller that can be built to adjust the motor frequency in a single direction (increasing) rather than in two (increasing and decreasing). Starting with a base frequency, the proportional component raises the motor frequency according to the error between a target compression ratio and the actual compression ratio. By itself, the proportional controller was found to be insufficient. The total controller is shown in Figure 96.

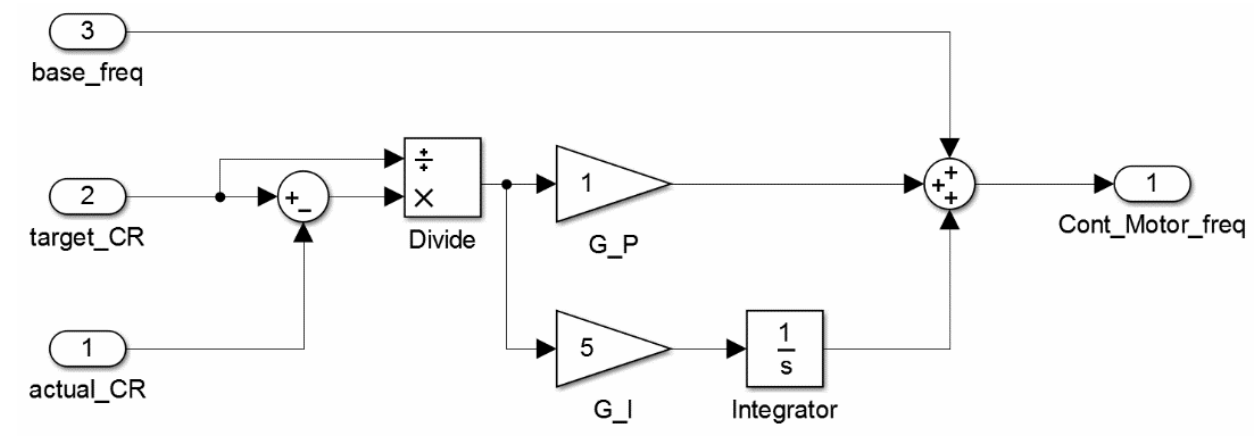

Figure 96: Motor controller subsystem in the Simulink model used for SALEG start-up.

By including the integral component, a moving bias (dependent on the accumulation of compression ratio error) is added to the base frequency along with the proportional component. Note that the compression ratio error is normalized by the target compression so that the controller gains are independent of system scale aside from motor frequency results. The relationship for the controller is presented in Eq. 69. 


$$
\begin{aligned}
& f_{\text {motor }}=f_{\text {motor, base }}+E+5 \int_{0}^{t} E d \tau \\
& E=\frac{C R_{\text {target }}-C R_{\text {actual }}}{C R_{\text {target }}}
\end{aligned}
$$

For the current SALEG device, a target compression ratio $\left(C R_{\text {target }}\right)$ of 19 is chosen because it exceeds the steady state performance compression, and the controller gains are set ane and five for the proportional and integral components, respectively. These values are shown to be effective, but are not optimized. Meanwhile, base motor frequency $\left(f_{\text {motor,base }}\right)$ can be varied parametrically to achieve different transient responses. The next two figures demonstrate a successful start-up given an initial motor frequency of $30 \mathrm{~Hz}$.
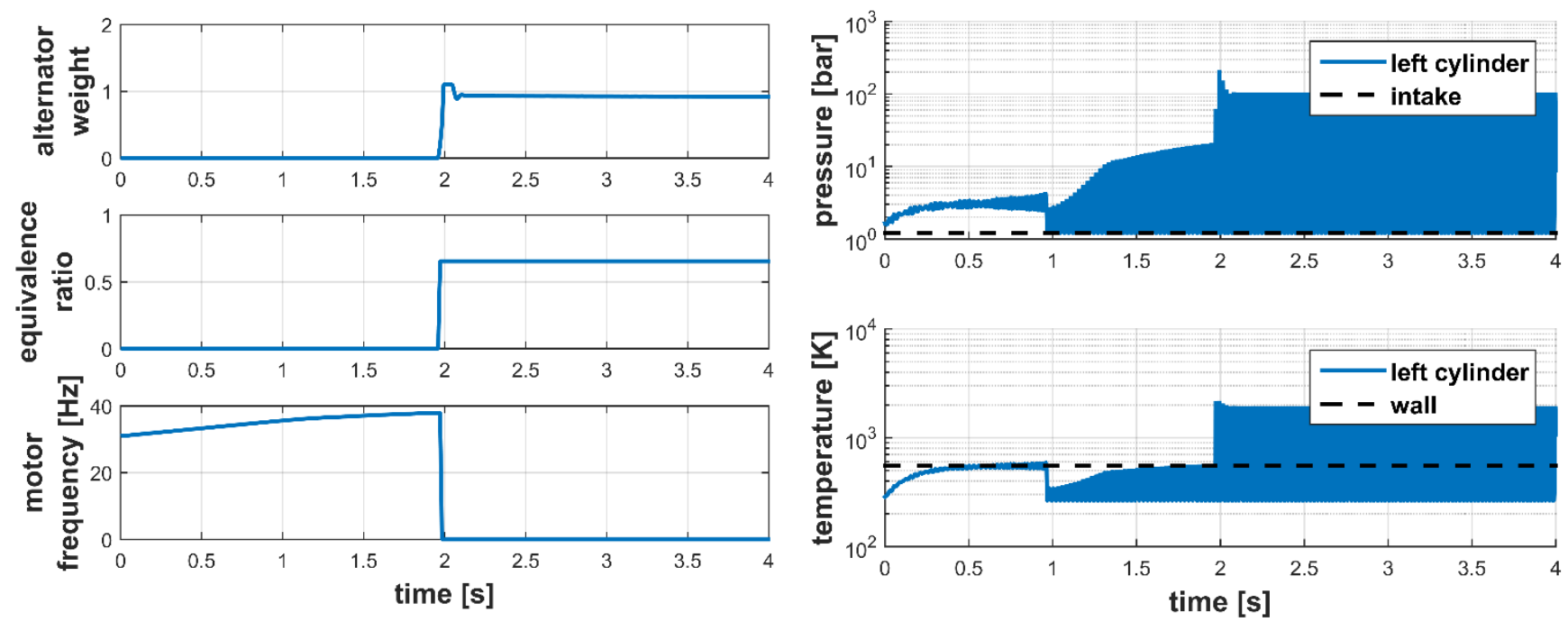

Figure 97: Controller outputs and engine cylinder transient response during start-up of the SALEG.

Figure 97 provides selected outputs from the controller in the left-hand plot. In the right-hand plot are the transient response of the left cylinder temperature and pressure for a successful start-up of the current SALEG with a base motor frequency of $30 \mathrm{~Hz}$ and motor force amplitude (peak force) of $264 \mathrm{~N}$. The alternator weight refers to the scaling factor applied to the alternator force by the alternator controller to encourage stability - a value of one corresponds to an unaffected alternator force while zero corresponds to no alternator force. The maximum alternator weight is limited by the physical alternator parameters, so a saturation limit of 1.1 is applied within the controller.

Both alternator weight and equivalence ratio rises coincide with the fall of motor frequency and rise in cylinder temperature and pressure of the right hand plots. These sudden changes, occurring just prior to two seconds, indicate the point at which motored operation transitions to fired operation. Prior to the transition, the motor frequency is slowly raised in accordance with controller demand. At the same time, the cylinder pressure and temperature undergo two distinct phases. Before the one second mark, 
the wall temperature causes the cylinder gases to heat and pressure to rise. These changes occur because the stroke length does not yet accommodate gas exchange. The sharp change near the one second mark occurs as the stroke length increases sufficiently to open the ports and allow gas exchange. Then, between one and two seconds, peak pressure and temperature rise according to the increase of compression ratio. Just before the two second mark, sufficient compression is achieved to cause fuel injection leading to combustion and sharp rise in peak pressure and temperature. The transient behavior of compression ratio and stroke are shown in Figure 98.
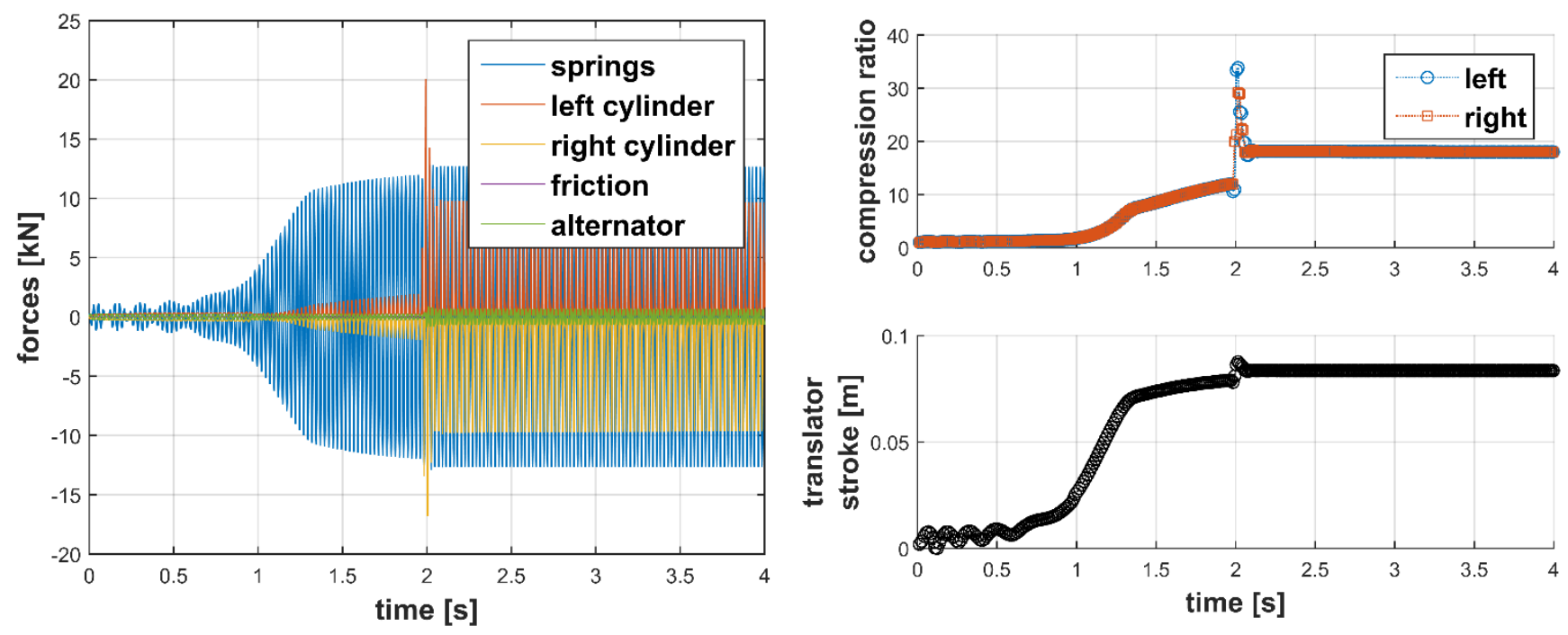

Figure 98: Forces acting on the translator, compression ratio, and stroke length during SALEG start-up.

Noting that the force from the springs is reflective of the translator position, early oscillatory motion is evident leading up to the point at which the resonant zone is entered, beginning around the one second mark and leading up to the transition point near two seconds. For this case, the total start-up time is under 2.5 seconds, and the steady state performance matches that seen in Table 13 and the preceding figures. Next, four different responses are given in Figure 99, each resulting from a different initial motor frequency. 


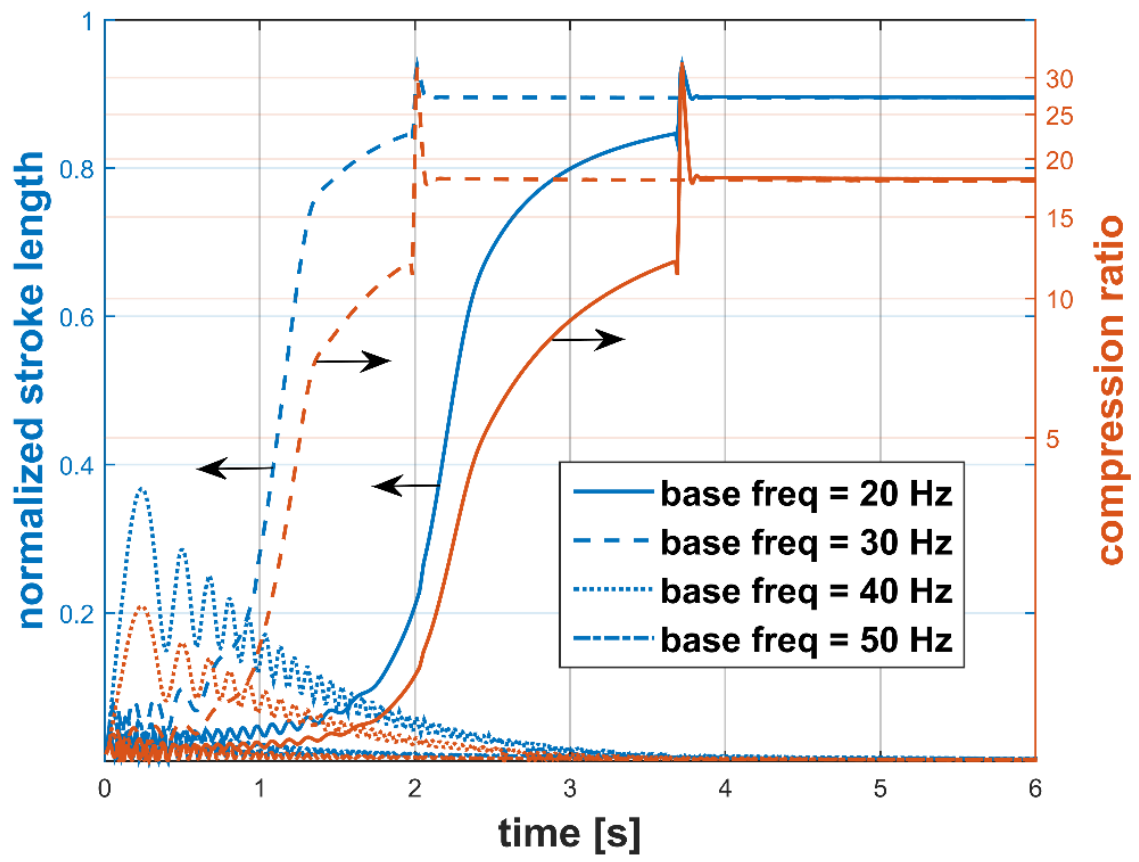

Figure 99: Variations in SALEG start-up response as initial motor frequency is varied.

Recalling that the resonant frequency for this simulated SALEG is just above $40 \mathrm{~Hz}$, both cases where the motor frequency begins sufficiently below the resonant zone result in successful start-up. The start-up time can be reduced by causing the base frequency to be closer to the resonant frequency. However, the third case - where the initial motor frequency is set to nearly match the resonant frequency - results in overshoot by the controller and unsuccessful start-up. Because the controller is designed only to raise the motor frequency, both the third case $(40 \mathrm{~Hz})$ which overshoots and the final case $(50 \mathrm{~Hz})$ that is clearly above the resonant frequency cannot result in successful start-up.

This section demonstrates two important concepts for start-up of the SALEG or likely any free piston device. First, the dynamic behavior of the translator is similar to a classical mass-spring-damper system wherein a natural frequency exists and resonance can occur when the frequency of the excitation force concurs. Second, a controller, dependent only on translator position, may be sufficient to "hot-start" the SALEG by employing its resonance to increase the compression ratio and stroke length until sufficient for combustion and steady state, fired operation. Alternative architectures for the controller as well as optimized controller parameters could improve the response of the SALEG. And, the controller would be made more robust if it could both raise and lower the motor frequency to achieve start-up regardless of the initial motor frequency.

\subsection{Response to Change in Load Demand}

During operation, an engine must be able to respond to changes in load. In the conventional engine, change in power output is accomplished by fuel and air management and results in a change of engine 
speed. Previously discussed in the literature review as a concern for the $\mathrm{HCCl}$ engine is its narrow operating range. The free piston engine has the potential to enlarge the envelope with the use of varied compression ratio, but requires a controller that can balance system stability against meeting load demand. For the steady state SALEG, performance is optimized for engine operation at peak power output. To maximize the power density, the alternator mover mass is minimized. Together these statements imply that maximum power draw by the alternator coincides with optimized power output. Allowing that maximum alternator power draw may exceed optimized power gives authority to the alternator controller to help maintain stability (as described in section 3.6.6) and leads to the overshoot seen in the previous start-up figures.

Load demand, however, may vary from the optimized load point and should be met so long as the demand is less than the maximum output of the alternator. The current SALEG device is optimized to provide $3.8 \mathrm{kWe}$ of power, but it is possible that the alternator would only be required to provide a percentage of that power. Two steps are taken to impose a change in load demand within the simulation space. The first is to supply the power demand signal in the alternator controller. The power demand signal can be altered as desired to simulate step changes, ramp changes, or duty cycles. The second is to build a controller that attempts to drive the alternator output towards meeting the performance target by affecting the alternator load constant. The power demand controller consists of both proportional and integral components, but is held secondary to the stability controller by a weighted mean of the two controller commands. The total alternator controller is referred to as the P+PI controller. Given that the current hot start routine achieves nominal maximum power output, decreasing step and ramp changes are applied within the simulation. The result of a decreasing step changes is seen in Figure 100.
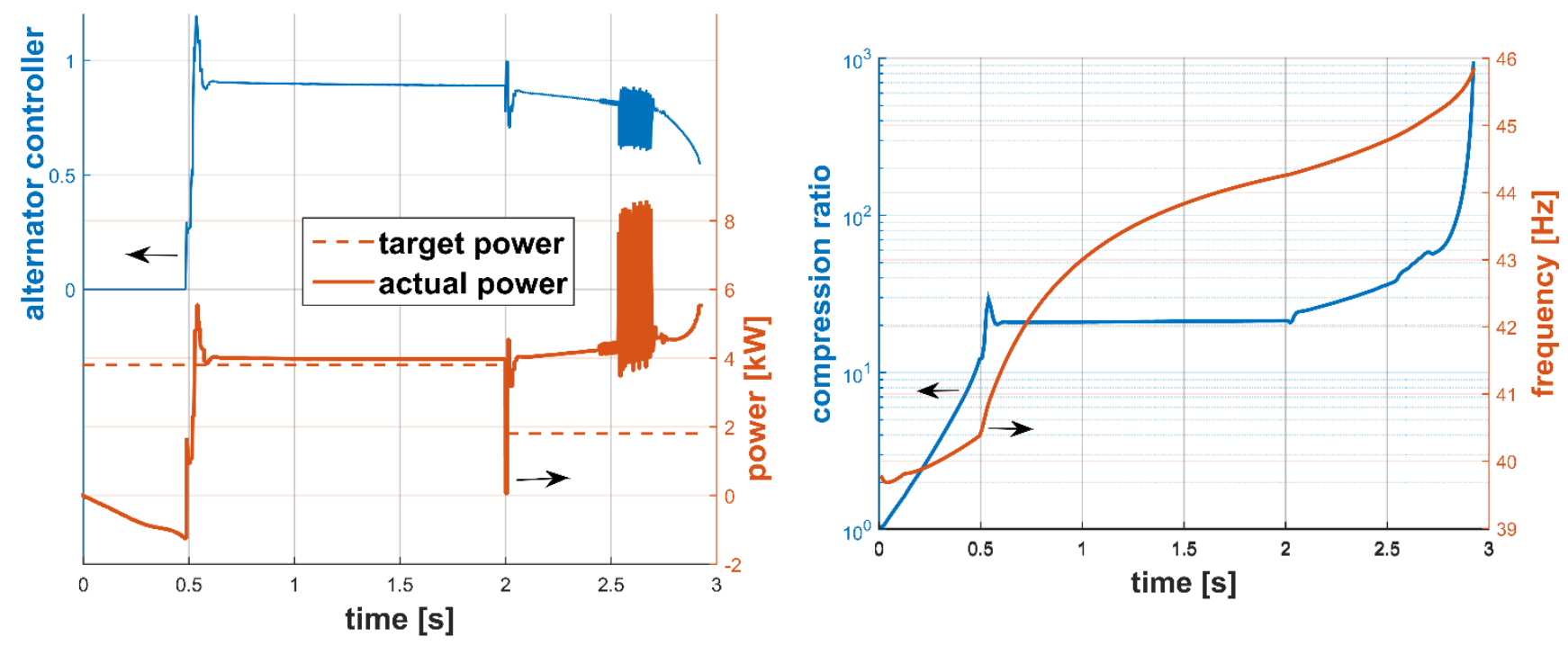

Figure 100: Response of the SALEG as it reacts to a step decrease in power demand. Runaway compression results from using only the alternator P+PI controller to respond to the change in load demand. 
The left-hand plot in Figure 100 demonstrates the mean power output of the alternator as a function of time along with the power demand and alternator P+PI controller command signal. The early start-up phase of the simulation is shown followed by a steady state operation phase. At the two second mark, a decreasing step change of $-2 \mathrm{kWe}$ is imposed. The $\mathrm{P}+\mathrm{PI}$ controller reacts slowly because the proportional gain on the demand component is kept low to aid in stability. Examining the right-hand plot of Figure 100 shows that the decreasing load command results in increasing compression ratio and engine speed. This leads to the unintended consequence of increased power output despite the controller signal. The system eventually grows unstable.

After extensive experimentation with gain values, the simple alternator $\mathrm{P}+\mathrm{PI}$ controller proved to be insufficient. This result is not surprising given the architecture of the $\mathrm{P}+\mathrm{PI}$ controller which attempts to maintain stability at a constant stroke length regardless of the desired power output. The dynamics of the SALEG demand that the applied load be matched to energy input through fuel and subsequent losses. This is a tenuous balance for the free piston, as demonstrated by the fundamental analysis and unstable regions seen throughout the parametric analysis. In short, an additional control variable is needed that responds to the load demand to affect the total operation of the SALEG.

The parametric sweeps also demonstrated the strong response of the SALEG to intake temperature. Returning to the literature, intake temperature is demonstrated as a possible control mechanism for $\mathrm{HCCl}$ combustion in a crankshaft driven engine [67] via the use of simulation to map the engine response to varied input conditions. A similar approach is taken here to drive the SALEG operation through intake temperature management. Figure 101 shows the power and compression ratio of the SALEG as functions of intake temperature.

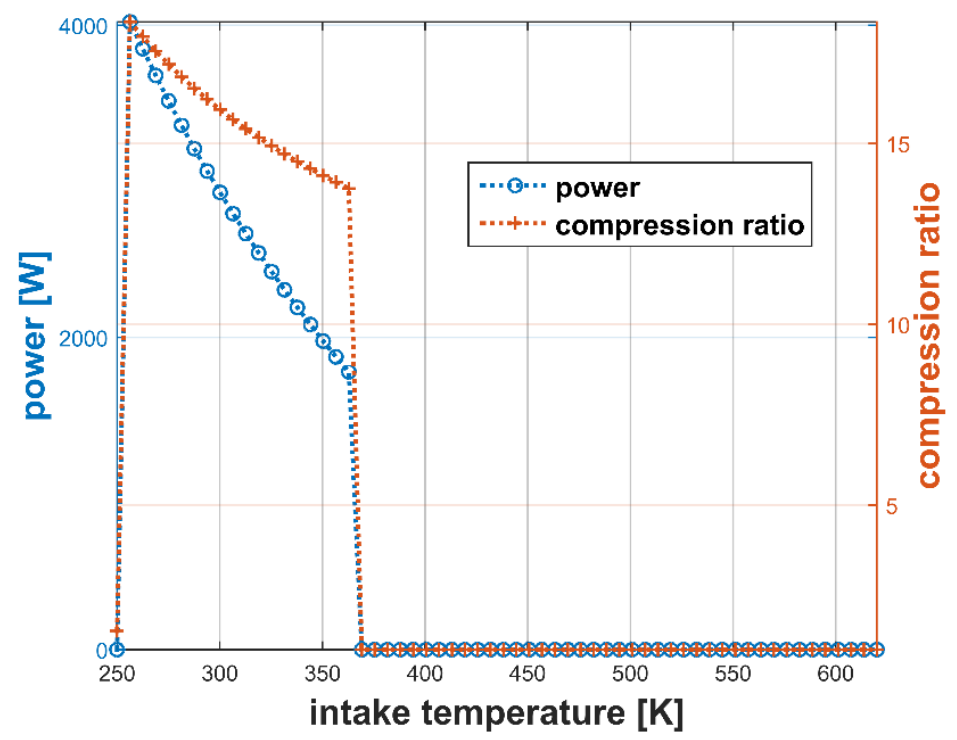

Figure 101: Parameter sweep of intake temperature showing the relationships between intake temperature, power, and compression ratio. 
To affect power output of the system, an intake temperature controller is constructed. Mapping between power demand and compression ratio provides the target compression for the SALEG. The error between target and actual compression then drives an integral controller to affect intake temperature. Because the map shows that the SALEG is limited to a specific range, the interpolation between power and compression ratio is clipped rather than extrapolated. For the Simulink representation of this controller, see Figure 113 in Appendix A. With the intake temperature controller included in the simulation, the same step down of power demand is applied. The results are given in Figure 102.
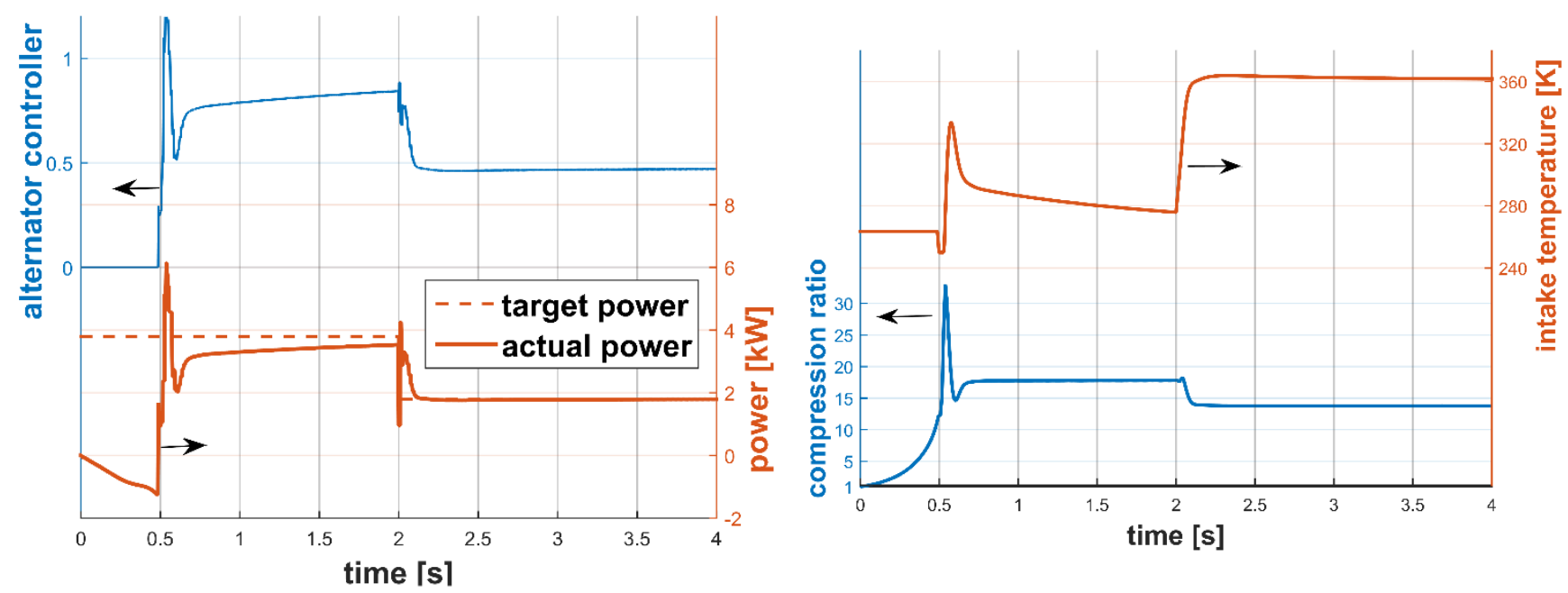

Figure 102: Successful response of the SALEG to a step decrease in load demand. Target compression ratio is matched to power demand and intake temperature is controlled to meet target compression ratio.

The ability of the intake temperature controller to meet the power demand is evident in Figure 102. The increase of intake temperature corresponds to the drop in compression ratio. This occurs because intake temperature causes earlier combustion and thus adverse work around top dead center. Both peak pressure and efficiency are reduced - with the system efficiency dropping to $22 \%$. Unfortunately, inclusion of the intake temperature controller also increases the time needed for the SALEG to achieve peak performance after start-up, as shown in the left-hand plot. Next, the response to ramp decrease in power demand is shown. 

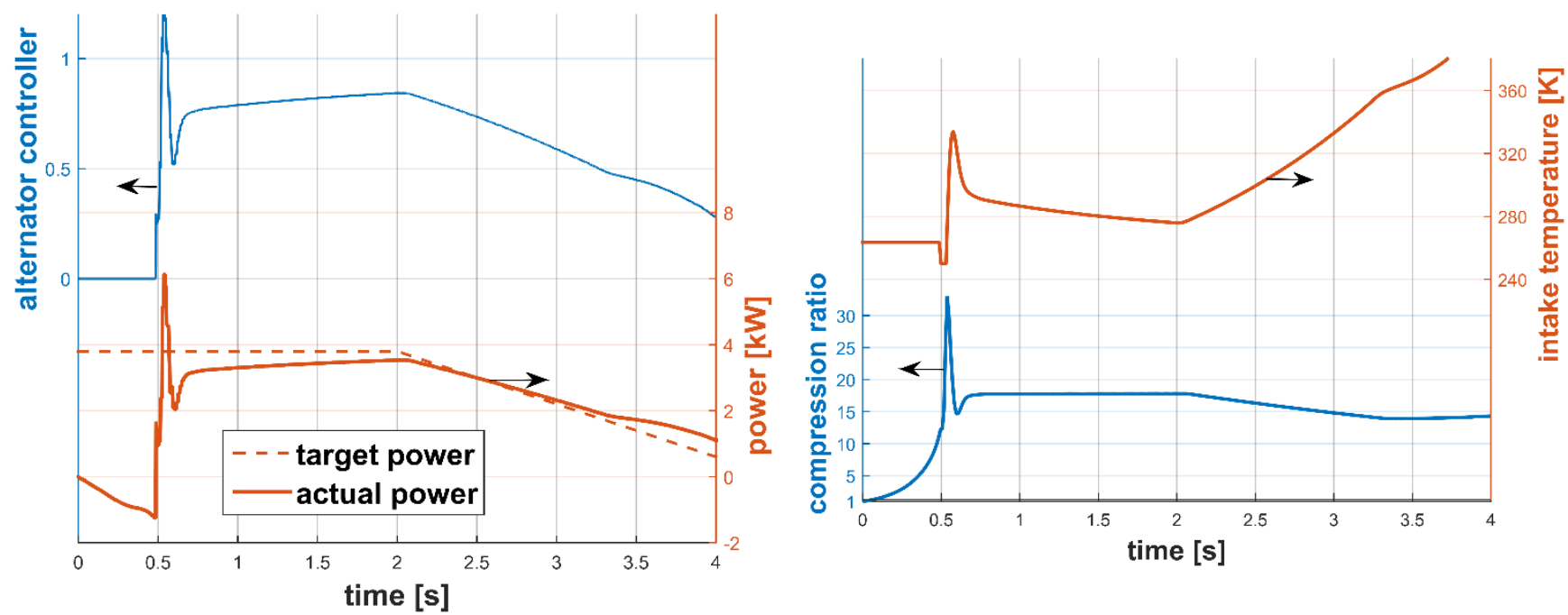

Figure 103: Successful response of the SALEG to a ramp decrease in load demand. Target compression ratio is matched to power demand and intake temperature is controlled to meet target compression ratio.

Figure 103 shows that the intake temperature controller responds well to the steadily decreasing demand of power until the power demand falls outside the stable range shown in Figure 101. For this low load operation, the intake controller is no longer sufficient. This low load operation also carries significance for efficiency which is reduced to $15 \%$ as compared to the $38 \%$ at full throttle.

While the intake temperature greatly improved the ability of the SALEG to respond to changes in load, it is limited in its ability. Practically, the intake temperature can be heated by the exhaust stream from the engine. Exhaust pulses are minimized in the SALEG because of the alternating two stroke cycles, but increasing intake temperature might still be considered a long term control variable with its effects not being fully realized for multiple cycles. Fuel control is the more immediate option for affecting engine operation. Control over fuel injection can be used to change engine operation within one cycle and is strongly related to power and compression ratio. 


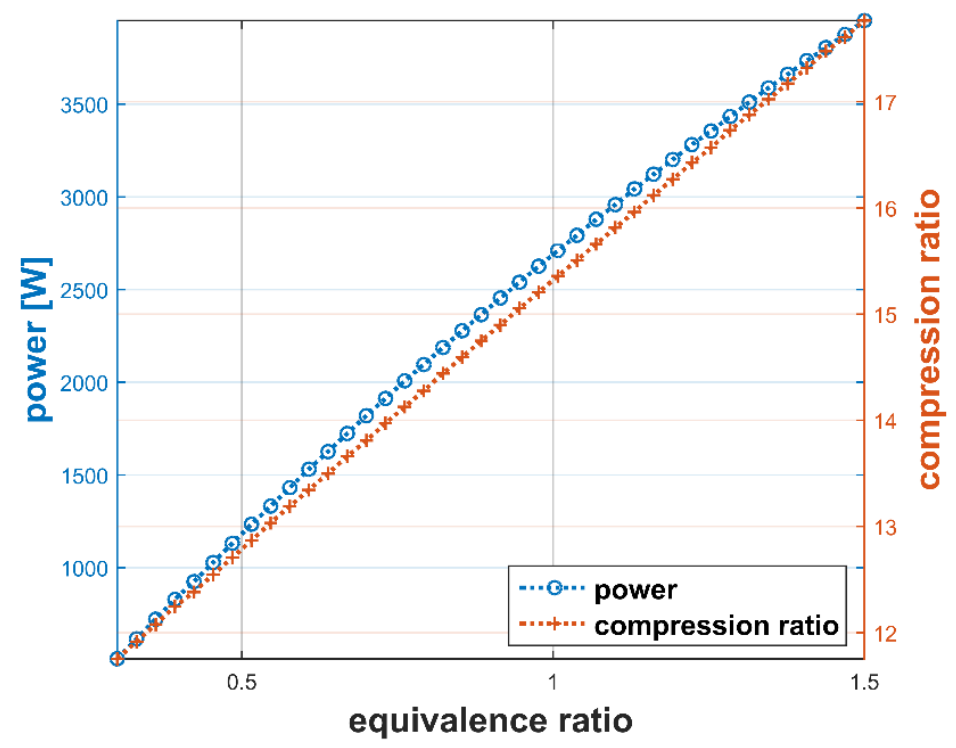

Figure 104: Relationship between equivalence ratio, power, and compression ratio.

Figure 104 shows power and compression ratio dependent on equivalence ratio for the base case SALEG except with intake temperature set to $370 \mathrm{~K}$, the limiting temperature in Figure 96. This demonstrates that stable operation can be achieved for output power of less than $1000 \mathrm{~W}$ at the maximum stable intake temperature. Extending the use of equivalence ratio, a map of stable operating points can be constructed for compression ratio and power as functions of both equivalence ratio and intake temperature. These maps are given in Figure 105.
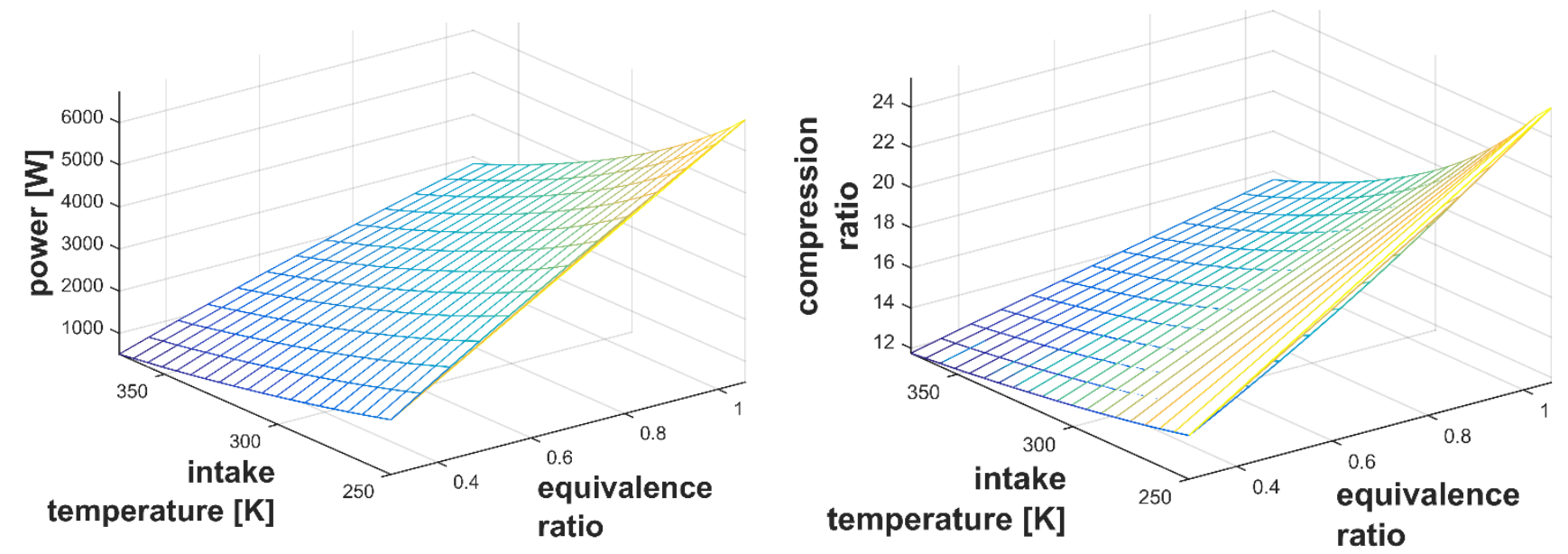

Figure 105: Intake temperature and equivalence ratio maps for power and compression ratio.

The maps shown in Figure 105 reveal that a significant amount of operating space is available for the current SALEG device. The relationship between power and compression remains consistent, however, so that power demand can be translated to target compression ratio which can then be used within a controller to drive either the equivalence ratio or intake temperature. Unfortunately, the combined use of intake temperature and equivalence ratio does not appear to offer benefits for efficiency, which is 
significantly reduced at part load. The strong relationship between power and compression ratio, and then between compression ratio and efficiency, may indicate that reduced power is tied to reduced efficiency.

\subsection{Recovery from Engine Misfire}

Simple misfire can be simulated in the Simulink model with the use of a step change applied to the fuel control that causes the fuel to be cut after a specified time. Combining this with the start-up might be said to represent a shortened duty cycle for the SALEG, and is illustrated in Figure 106. Demonstrated are three modes of operation. The first is start-up wherein compression ratio, stroke length, and frequency are raised as the motor frequency is controlled to match resonance. The initial motor frequency is set to $38 \mathrm{~Hz}$ to achieve rapid start-up, and only the alternator proportional stability controller is active. The alternator power shows increasing negative power during the first mode indicating that the alternator is operating as a motor and that power input by the alternator is increasing with translator speed. The second mode is steady state operation with constant fuel and power output. Frequency has a relatively long rise time compared to compression ratio and stroke length.
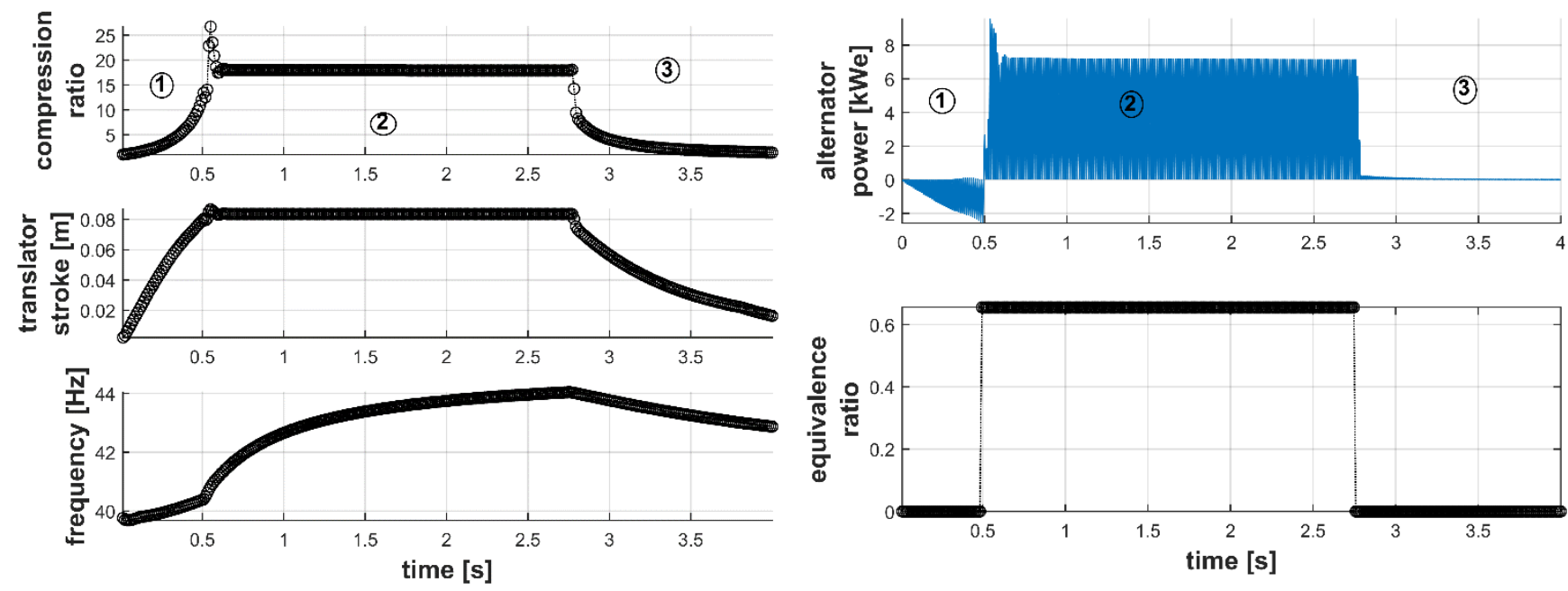

Figure 106: Simulated short duty cycle of SALEG with motored resonant start-up, steady state operation, and ending with fuel being cut.

The final mode is after fuel has been cut. Looking closely at the power plot, there is a short transition period between full power draw and no power draw as the alternator controller reacts to the sudden lack of fuel input. Through the remainder of the third mode is translator "ring-down" as friction dampens the translator motion. It is notable that the fall-off of compression is very steep after misfire, indicating that recovery depends on rapid controller response. Another condition that might lead to misfire is excessive load and loss of compression. This case is presented in Figure 107. 

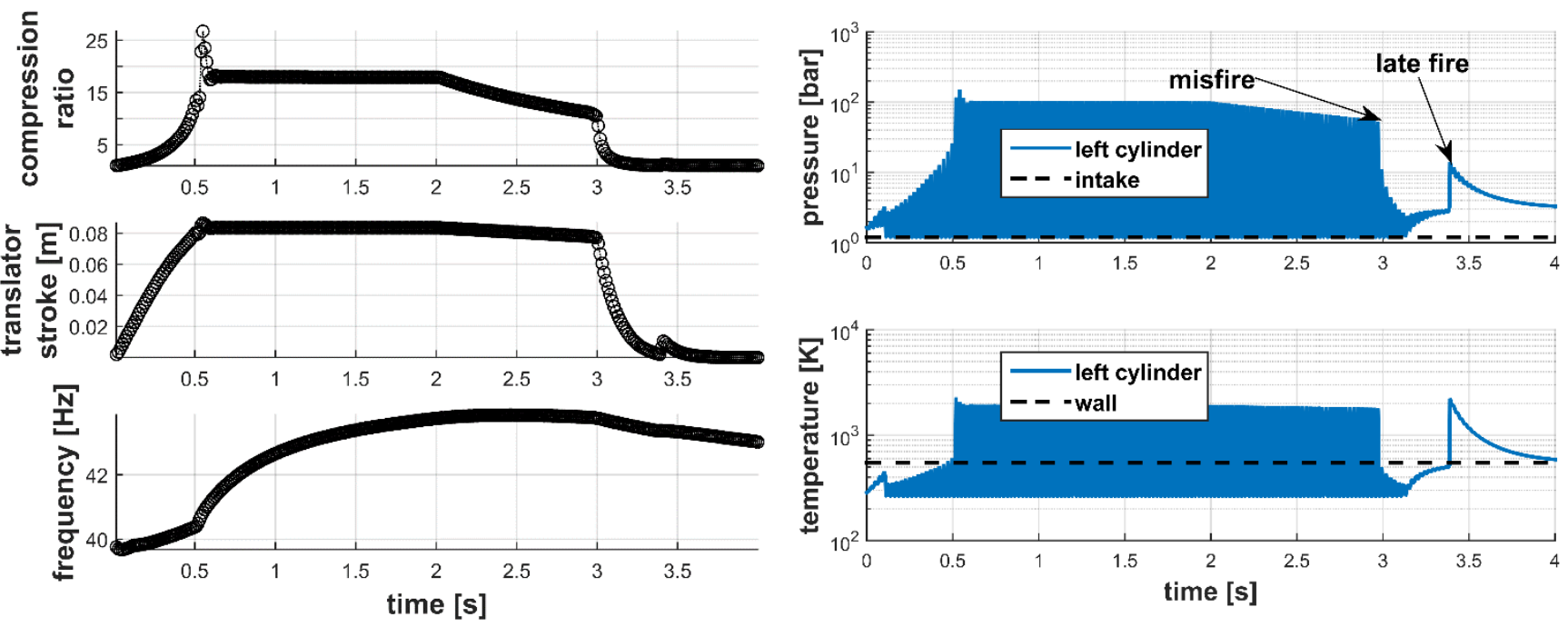

Figure 107: Steadily increasing alternator load applied to the SALEG translator, eventually resulting in engine misfire. Rapid loss of compression follows the misfire and results in engine ring-down.

To simulate the excessive load scenario, a ramp increase was applied to the load resulting in the ramp decrease in compression occurring between two and three seconds. Near three seconds, the compression ratio falls below 10 and misfire occurs. The steep drop-off of compression ratio and stroke follow. The pressure and temperature plots for the left cylinder show this drop-off, along with the ringdown of the translator. Because the alternator controller cuts the power draw after compression ratio is sufficiently low, friction is the only damping component. After the ports close, the hot cylinder walls act to heat the cylinder charge and raise the cylinder pressure. A latent combustion event is predicted by the CFR ignition model causing a spike in cylinder energy and translator stroke. Regardless, the SALEG continues to lose energy and would eventually stall without motor excitation.

Both of these cases demonstrate the need for rapid recovery of translator oscillation after the occurrence of misfire. Intake temperature was used successfully to control the power output of the stroke because of its ability to advance the combustion timing. Unfortunately, the gas exchange process for one engine cylinder coincides with the combustion (or misfire) of the other, rendering the intake temperature useless as an immediate recovery tool. This same reasoning applies to fuel injection, thus eliminating all except the alternator controller as a candidate for recovery of the engine after misfire.

A simple method for recovery may be to fall back on the resonant start-up routine if the compression ratio falls below a given threshold. As in the start-up study, this threshold is twelve. The control logic is implemented within the state model of the controller, and the results are demonstrated in Figure 108. 

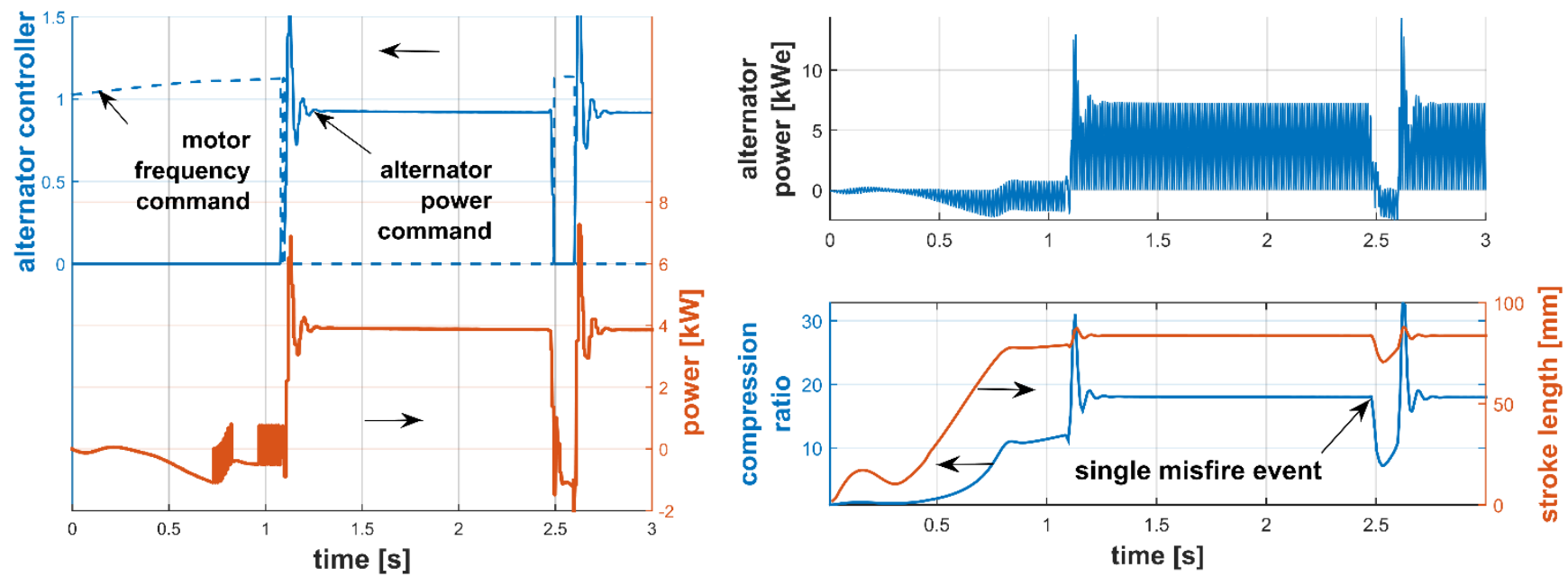

Figure 108: Transient response and recovery of SALEG stable operation after misfire via the resonant start-up controller.

Misfire is imposed within the simulation space by cutting the fuel for a single engine stroke. Rapid loss of compression activates the alternator inverter which then causes a force to be applied to the translator just as in the start-up sequence. The motor frequency is increased to the point of resonance, and steady state operation is recovered within half of a second after the misfire occurs.

\subsection{Conclusions Regarding Control}

Through the last three sections, some basic control methodology has been explored for the SALEG. Free piston resonance was demonstrated to be a viable method for both initial start-up of the device (it is expected that both hot-start and cold-start could be achieved) and recovery after misfire. Partial load performance is achieved with the use of intake temperature management and performance mapping between desired power and compression ratio. It is shown that the same effects could be achieved with similar mapping between equivalence ratio and desired power. While these tools are effective within the given simulation space, no optimization of controller parameters is sought, and many more paths to successful control might be available. This becomes especially true if the engine model is enhanced with valves that can be actuated on command, direct fuel injection, or spark/glow plugs in the engine cylinders for combustion.

Despite the overall robustness demonstrated by the Simulink model, the controllers presented here command simple models that do not represent the physical systems with accuracy. For instance, the intake temperature command is met instantaneously within the perfect gas exchange cylinder model and the alternator inverter model consists of a mathematical sign change rather than modeled electronics. These represent areas where increased model sophistication might prove the simple control methods to be inadequate. 
Of particular interest in the area of control is a method to increase efficiency at part load. It was shown that power, compression ratio, and efficiency may be too closely tied for high efficiency at low load. However, full mapping of the system and sophisticated control architecture may provide avenues to maintain high efficiency at low load, even if that efficiency is below that of peak load operation. The sophisticated control architecture might be based on the system mapping or could consist of model based components with predictive capabilities. Though not pursued in this investigation, it may be possible to predict an event such as misfire and to take early preventative action to prevent the misfire or prepare for rapid recovery. It is also possible that a controller, built to include knowledge of the $\mathrm{HCCl}$ combustion parameters, could tune the combustion performance to match the dynamics of the translator for high efficiency over a wider load range. 


\section{CH. 6 THE LAST CHAPTER}

\subsection{Summary and Conclusions}

The dual free piston HCCI SALEG presented in this dissertation has the potential to succeed in a very competitive energy market because of its simplicity, energy density, and high efficiency. Realizing this potential depends on research and development of the device on many fronts. As a prelude to prototype testing of the SALEG, a series of simulation based investigations has been presented to advance the understanding of the device and the surrounding concepts.

This dissertation contributes to advancing the free piston linear engine in three primary areas. The first is evaluation of the behavior of the SALEG through fundamental and numerical simulation. To achieve this, a theoretical, closed form solution is presented to show the fundamental nature of the SALEG. This is followed by the development and testing of a numeric simulation tool that has been extensively used to study the behavior of the SALEG during steady state and transient operation. Testing of the model provides important understanding of the free piston behavior, and compares the common modeling approaches seen in the literature. Using MATLAB ${ }^{\circledR}$ and Simulink, the virtual model is able to be modified to accommodate many research goals. The natural modularity of Simulink is employed so that the model architecture is well segregated and more easily understood. Each of the various processes within the model can be altered or enhanced to augment the accuracy or purpose of the simulation. And the model could be adapted to simulate a variety of free piston linear engine configurations and functions. Most importantly, it provides a foundation for future endeavors that might involve experimental data and improvement of the simulation by correlation.

The model is then tested with respect to the underlying mathematical relationships. This serves to test its sensitivity to the numerical methodology and modeling options found throughout the literature. The model is verified against concepts and data found in the literature, but the predictive quality of the simulation does not account for many of the impactful complexities of a real device. One of the most important of these is the combustion event. Sensitivity of the numeric model to combustion profile, duration, and ignition timing is demonstrated, and duration and timing are shown to significantly impact steady state performance. However, the $\mathrm{HCCl}$ combustion model is greatly limited with respect to these findings. The 1-D pressure model does not characterize cylinder charge stratification; ignition timing is predicted by a model not correlated to the free piston engine; and combustion efficiency and combustion duration are fixed for a given simulation, independent of device behavior and conditions in the cylinder. Other simplifications further limit the simulation accuracy, but the model retains its usefulness as a tool to study trends and behavior of the SALEG and as a platform for future research. 
The second contribution is greater understanding of the SALEG design space. This is achieved through parametric and optimization studies using the SALEG simulation tool. The numeric model input parameters are reduced by including geometry and translator mass within the simulation loop so that stable, steady state operation depends on energy balance as well as reasonable component design. The simulation tool and parametric study serve as a platform for the optimized design of the SALEG through the use of a genetic algorithm. The optimization routine is constructed to maximize efficiency for a given target power with constraints applied to ensure a stable design with reasonable compression ratio and peak pressure. A table of optimized designs is presented, and the design space is illustrated with multidimensional scatter plots. While the simulated design space affords a wide range for optimization, the HCCI SALEG benefits from low intake temperature ( $300 \mathrm{~K})$ and low maximum stroke to bore ratio ( 1.2). Reducing the intake temperature enables higher compression, higher cylinder energy, and higher efficiency. Low stroke to bore ratio allows higher frequency and greater power density.

The parametric study shows that raising the spring stiffness might actually degrade system performance if doing so severely increases system inertia. This points toward the need for careful consideration of spring design. Spring design will need to achieve high spring stiffness with low inertia to maximize frequency and power density. These advances in understanding of the design space will be instrumental for advancing the commercial viability of the free piston engine.

The third and final contribution presented in this dissertation is greater understanding of transient operation and then the demonstration of suitable control mechanisms achieve start-up, meet load demand, and recover stable oscillation after misfire, within the simulation space. Hot start is successfully modeled with the alternator inverted to act as a motor and compel the motion of the translator. By matching the motor switching frequency to the natural resonance of the free piston SALEG, compression ratio and stroke are raised sufficiently to enable combustion. After combustion, the alternator inverter is deactivated and the controller switches to alternator mode where a light proportional controller helps to maintain stability at peak, steady state operation. The simulated start-up demonstrated in this dissertation serves as additional proof of concept for the use of resonance for start-up of the free piston engine, and encourages further testing in prototype devices.

Step and ramp changes in load demand are applied to the SALEG to investigate reduced load operation. By itself, the alternator controller is unable to reduce system output power. Alone, the reduction in load results in runaway compression. Instead, the output power of the SALEG must be matched to the engine performance. So, an intake temperature controller is established from mapped relationships between intake temperature, power output, and compression ratio. The results are successful response of the SALEG to change in load demand for a given range of intake temperature at the cost of efficiency. It is also shown that equivalence ratio (or more generally, the amount of fuel) can be controlled to achieve 
similar results, but it is not clear if the approach can benefit the system in terms of higher efficiency at part load. The combined use of equivalence ratio and intake temperature could likely extend the ability of the SALEG to respond to any load demand and further sophistication may be necessary to achieve high efficiency at low load.

The final controller demonstration consists of recovery from the misfire. Despite the inclusion of relatively stiff springs (evident by the elliptical shape of the position-velocity profile), misfire still results in very rapid loss of compression and eventual stall. Thus, the resonant start-up mechanism is employed to recover stable oscillation. While it may not be ideal to motor the SALEG during practical operation, it was shown to be an effective solution within the given simulation space.

\subsection{Recommendations for Future Research}

The leading recommendation to improve the quality of any research stemming from this dissertation is further improvement and validation of the numeric model. Accuracy of the single zone cylinder model is greatly tied to the supporting empirical correlations and simplifications. Improvements for each process of the two stroke cycle should be pursued, especially including more accurate models of ignition timing, combustion duration, combustion efficiency, and gas exchange. The complexity of these processes are grossly neglected in this dissertation and deserve attention due their influence on engine performance.

Additionally, a more accurate alternator model could be implemented and should be based on experimental data or finite element analysis to attain accurate values for model parameters. It would also be beneficial to investigate the influences of cogging forces and end effects on the transience of the SALEG as well as thermal effects on the alternator.

While the simulated design process proved useful for demonstrating the impact of selected design parameters, it involved many simplifications as well. Of particular interest is the design and implementation of mechanical springs. The parametric study brought to light the importance of reducing the inertia of the springs, showing that the frequency gained by increased spring stiffness might then be lost by the increase of inertia. The finding does not eliminate the usefulness of springs, but rather supports the innovative implementation of spring devices. Air springs (bounce chambers) might be the solution to this difficulty, and the increased complexity also affords additional control capabilities. However, if simplicity (and reduced cost) are to be maintained, the traditional coil spring might be avoided in favor of a unique design that provides stiffness while minimizing mass. Regardless, spring design for use within a free piston linear engine deserves further investigation.

Finally, limited control of the SALEG has been demonstrated in this dissertation. The use of resonance as a start-up strategy has excellent potential, and was shown to be achieved simply and with low power requirements. While this start-up investigation focused on hot-start due to the complexity of 
thermal transience, the resonance of an actual free piston device should be examined for a range of temperature conditions. The ability to sufficiently increase stroke and compression under resonant operation has yet to be validated on a physical device, and would be a large step towards successful start-up.

Control over the SALEG has also been demonstrated for changes in load demand and engine misfire. Responding to low load operation required reduced compression ratio, and thus incurred a penalty on efficiency. To avoid the loss, more sophisticated control might make use of parameter mapping or model-based control to find and drive operation towards the highest possible efficiency for the given load. Similar control methods could also prove powerful for predicting misfire or low efficiency combustion. These predictions could be used to take precautionary or preventative action against device failure. Such control sophistication would certainly require either experimental data or advanced computational methods capable of accurately predicting engine cylinder behavior. 


\section{REFERENCES}

[1] V. Etacheri, R. Marom, R. Elazari, G. Salitra, and D. Aurbach, "Challenges in the Development of Advanced Li-ion Batteries: A Review," Energy \& Environmental Science, 4(9): 3243-3262, 2011.

[2] M. Twigg, "Progress and Future Challenges in Controlling Automotive Exhaust Gas Emissions," Applied Catalysis B-Environmental, 70(1-4): 2-15, 2007.

[3] F. Kock, J. Haag, and H. Friedrich, "The Free Piston Linear Generator - Development of an Innovative, Compact, Highly Efficient Range-Extender Module," SAE Technical Paper 2013-011727, 2013.

[4] M. Robinson and N. Clark, "Fundamental Analysis of Spring-Varied, Free Piston, Otto Engine Device," SAE International Journal of Engines, 7(1): 195-220, 2014.

[5] M. Robinson and N. Clark, "Fundamental Explorations of Spring-Varied, Free Piston Linear Engine Devices," Journal of Engineering for Gas Turbines and Power, 137(10), 2015.

[6] M. Robinson and N. Clark, "Effect of Combustion Timing and Heat Loss on Spring-Assisted Linear Engine Translator Motion," Under Review for SAE World Congress 2016.

[7] M. Robinson, N. Clark, and P. Famouri, "Resonance of a Spring Opposed Free Piston Linear Engine Device," Under Review for SAE World Congress 2016.

[8] H. Aichlmayr, "Design Considerations, Modeling, and Analysis of Micro-Homogeneous Charge Compression Ignition Combustion Free-Piston Engines," Ph.D. Dissertation, University of Minnesota, Minneapolis, MN, 2002.

[9] R. Mikalsen and A. Roskilly, "A Review of Free-Piston Engine History and Applications," Applied Thermal Engineering, 27(14-15): 2339-2352, 2007.

[10] T. Callahan and S. Ingram, "Free Piston Engine Linear Generator for Hybrid Vehicles Modeling Study," Center for Electromechanics at The University of Texas, Austin, TX, US Army Report 7540-01-280-5500, 1995.

[11] P. Van Blarigan, N. Paradiso, and S. Goldsborough, "Homogeneous Charge Compression Ignition with a Free Piston: A New Approach to Ideal Otto Cycle Performance," SAE Technical Paper 982484, 1998.

[12] S. S. Goldsborough and P. Van Blarigan, "A Numerical Study of a Free Piston IC Engine Operating on Homogeneous Charge Compression Ignition Combustion," SAE Technical Paper 1999-01-0619, 1999.

[13] P. Van Blarigan, "Advanced Internal Combustion Electrical Generator," in Proceedings of the 2001 DOE Hydrogen Program Review, Livermore, CA, 2001. 
[14] S. Goldsborough and P. Van Blarigan, "Optimizing the Scavenging System for a Two-Stroke Cycle, Free Piston Engine for High Efficiency and Low Emissions: A Computational Approach," SAE Technical Paper 2003-01-0001, 2003.

[15] P. Koning, (2014, March 26), Free-Piston Engines: A Possible Route to Hybrid-Electric Vehicles. Available: http://crf.sandia.gov/free-piston-engines-a-possible-route-to-hybrid-electric-vehicles/

[16] N. Clark, S. Nandkumar, C. Atkinson, R. Atkinson, T. McDaniel, S. Petreanu, P. Famouri, and W. Cawthorne, "Operation of a Small Bore Two-Stroke Linear Engine," in ASME Internal Combustion Engine Fall Technical Conference, Clymer, NY, 33-42, 1998.

[17] C. Atkinson, S. Petreanu, N. Clark, R. Atkinson, T. McDaniel, S. Nandkumar, and P. Famouri, "Numerical Simulation of a Two-Stroke Linear Engine-Alternator Combination," SAE Technical Paper 1999-01-0921, 1999.

[18] E. Shoukry, S. Taylor, N. Clark, and P. Famouri, "Numerical Simulation for Parametric Study of a Two-Stroke Direct Injection Linear Engine," SAE Technical Paper 2002-01-1739, 2002.

[19] C. Tóth-Nagy, "Linear Engine Development for Series Hybrid Electric Vehicles," Ph.D. Dissertation, West Virginia University, Morgantown, WV, 2004.

[20] C. Tóth-Nagy and N. Clark, "The Linear Engine in 2004," SAE Technical Paper 2005-01-2140, 2005.

[21] C. Tóth-Nagy, P. Famouri, and N. Clark, "Compression Ignition Linear Engine Design Variable Effects," in Proceedings of the ASME Internal Combustion Engine Division Fall Technical Conference, Morgantown, WV, 1029-1036, 2012.

[22] ARPA-E, (2015, July 2), Generators for Small Electrical and Thermal Systems (GENSETS). Available:

http://arpae.energy.gov/sites/default/files/documents/files/GENSETS\%20Project\%20Descriptions FINAL v2.pdf

[23] R. Mikalsen and A. Roskilly, "Performance Simulation of a Spark Ignited Free-Piston Engine Generator," Applied Thermal Engineering, 28(14-15): 1726-1733, 2008.

[24] R. Mikalsen and A. Roskilly, "The Design and Simulation of a Two-Stroke Free-Piston Compression Ignition Engine for Electrical Power Generation," Applied Thermal Engineering, 28(5-6): 589-600, 2008.

[25] R. Mikalsen and A. Roskilly, "A Computational Study of Free-Piston Diesel Engine Combustion," Applied Energy, 86(7-8): 1136-1143, 2009.

[26] R. Mikalsen and A. Roskilly, "The Control of a Free-Piston Engine Generator. Part 1: Fundamental Analyses," Applied Energy, 87(4): 1273-1280, 2010. 
[27] R. Mikalsen and A. Roskilly, "The Control of a Free-piston Engine Generator. Part 2: Engine Dynamics and Piston Motion Control," Applied Energy, 87(4): 1281-1287, 2010.

[28] M. Hanipah, R. Mikalsen, and A. Roskilly, "Recent Commercial Free-Piston Engine Developments for Automotive Applications," Applied Thermal Engineering, 75: 493-503, 2015.

[29] Z. Zhao, F. Zhang, C. Zhao, and Y. Chen, "Modeling and Simulation of a Hydraulic Free Piston Diesel Engine," SAE Technical Paper 2008-01-1528, 2008.

[30] Z. Feng, Y. Huang, F. Zhang, C. Zhao, and K. Han, "Experimental Study on Hydraulic Free Piston Diesel Engine," SAE Technical Paper 2010-01-2149, 2010.

[31] J. Mao, Z. Zuo, and H. Feng, "Dimensionless Parametric Analysis of Spark Ignited Free-Piston Linear Alternator," in Thermodynamics - Interaction Studies - Solids, Liquids and Gases, Ch. 10, Ed: J. Moreno-Pirajan, ISBN 978-59533075631, 2011.

[32] C. Tian, H. Feng, and Z. Zuo, "Load Following Controller for Single Free-Piston Generator," Mechatronics and Applied Mechanics, 157-158(1-2): 617-621, 2012.

[33] B. Jia, Z. Zuo, H. Feng, G. Tian, and A. Roskilly, "Development Approach of a Spark-Ignited FreePiston Engine Generator," SAE Technical Paper 2014-01-2894, 2014.

[34] B. Jia, Z. Zuo, G. Tian, H. Feng, and A. Roskilly, "Development and Validation of a Free-piston Engine Generator Numerical Model," Energy Conversion and Management, 91: 333-341, 2015.

[35] H. Feng, Y. Song, Z. Zuo, J. Shang, Y. Wang, and A. Roskilly, "Stable Operation and Electricity Generating Characteristics of a Single-Cylinder Free Piston Engine Linear Generator: Simulation and Experiments," Energies, 8(2): 765-785, 2015.

[36] Y. Li, Z. Zuo, H. Feng, and B. Jia, "Parameters Matching Requirements for Diesel Free Piston Linear Alternator Start-Up," Advances in Mechanical Engineering, 7(3): 8, 2015.

[37] F. Kock and C. Ferrari, "Flatness-Based High Frequency Control of a Hydraulic Actuator," ASME Journal of Dynamic Systems Measurement and Control, 134(2): 7, 2012.

[38] J. Haag, C. Ferrari, J. Starcke, M. Stöhr, and U. Riedel, "Numerical and Experimental Investigation of In-Cylinder Flow in a Loop-Scavenged Two-Stroke Free Piston Engine," SAE Technical Paper 2012-32-0114, 2012.

[39] F. Rinderknecht, "A Highly Efficient Energy Converter for a Hybrid Vehicle Concept - Focused on the Linear Generator of the Next Generation," in $8^{\text {th }}$ International Conference and Exhibition on Ecological Vehicles and Renewable Energies, Monte-Carlo, France, 1-7, 2013.

[40] F. Rinderknecht, "Iron Losses of a Linear Generator for a Hybrid Vehicle Concept," in $4^{\text {th }}$ International Conference on Power Engineering, Energy and Electrical Drives, Istanbul, Turkey, 252-257, 2013. 
[41] S. Schneider, F. Rinderknecht, and H. Friedrich, "Design of Future Concepts and Variants of the Free Piston Linear Generator," in $9^{\text {th }}$ International Conference on Ecological Vehicles and Renewable Energies, Monte-Carlo, France, 1-8, 2014.

[42] D. Carter and E. Wechner, "The Free Piston Power Pack: Sustainable Power for Hybrid Electric Vehicles," SAE Technical Paper 2003-01-3277, 2003.

[43] J. Fredriksson and I. Denbratt, "Simulation of a Two-Stroke Free Piston Engine," SAE Technical Paper 2004-01-1871, 2004.

[44] A. Kleemann, J. Dabadie, and S. Henriot, "Computational Design Studies for a High-Efficiency and Low-Emissions Free Piston Engine Prototype," SAE Technical Paper 2004-01-2928, 2004.

[45] H. Kosaka, T. Akita, K. Moriya, S. Goto, Y. Hotta, T. Umeno, and K. Nakakita, "Development of Free Piston Engine Linear Generator System Part 1 - Investigation of Fundamental Characteristics," SAE Technical Paper 2014-01-1203, 2014.

[46] S. Goto, K. Moriya, H. Kosaka, T. Akita, Y. Hotta, T. Umeno, and K. Nakakita, "Development of Free Piston Engine Linear Generator System Part 2 - Investigation of Control System for Generator," SAE Technical Paper 2014-01-1203, 2014.

[47] N. Hung, O. Lim, and N. lida, "The Effects of Key Parameters on the Transition From SI Combustion to $\mathrm{HCCl}$ Combustion in a Two-Stroke Free Piston Linear Engine," Applied Energy, 137: 385-401, 2015.

[48] Y. Oh, O. Lim, G. Kim, and N. lida, "A Study for Generating Power on Operating Parameters of Powerpack Utilizing Linear Engine," SAE Technical Paper 2012-32-0061, 2012.

[49] J. Lee, O. Lim, and G. Kim, "An Experimental Study on the Effects of Spring Stiffness on the Combustion and Dynamic Characteristics of a Linear Engine," Journal of Mechanical Science and Technology, 28(5): 1945-1950, 2014.

[50] I. Boldea and S. Nasar, "Permanent-Magnet Linear Alternators Part 1: Fundamental Equations," IEEE Transactions on Aerospace and Electronic Systems, 23(1): 73-78, 1987.

[51] I. Boldea and S. Nasar, "Permanent-Magnet Linear Alternators Part II: Design Guidelines," IEEE Transactions on Aerospace and Electronic Systems, 23(1): 79-82, 1987.

[52] S. Nasar and C. Chen, "Optimal-Design of a Tubular Permanent-Magnet Linear Alternator," Electric Machines and Power Systems, 14(3-4): 249-259, 1988.

[53] S. Nasar and C. Chen, "Magnetic-Circuit Analysis of a Tubular Permanent-Magnet Linear Alternator," Electric Machines and Power Systems, 13(6): 361-371, 1987.

[54] R. Pai, I. Boldea, and S. Nasar, "A Complete Equivalent-Circuit of a Linear Induction-Motor with Sheet Secondary," IEEE Transactions on Magnetics, 24(1): 639-654, 1988. 
[55] G. Dawson, A. Eastham, J. Gieras, R. Ong, and K. Ananthasivam, "Design of Linear Induction Drives by Field Analysis and Finite-Element Techniques," IEEE Transactions on Industry Applications, 22(5): 865-873, 1986.

[56] J. Zou, M. Zhao, Q. Wang, J. Zou, and G. Wu, "Development and Analysis of Tubular Transverse Flux Machine with Permanent-Magnet Excitation," IEEE Transactions on Industrial Electronics, 59(5): 2198-2207, 2012.

[57] A. Souissi, M. Zouaghi, I. Abdennadher, and A. Masmoudi, "MEC-Based Modeling and Sizing of a Tubular Linear PM Synchronous Machine," IEEE Transactions on Industry Applications, 51(3): 2181-2194, 2015.

[58] Q. Lu and Y. Ye, "Design and Analysis of Tubular Linear PM Generator," IEEE Transactions on Magnetics, 45(10): 4716-4719, 2009.

[59] T. Dang, P. François, L. Prévond, and H. Ahmed, "Theoretical and Experimental Results of Tubular Linear Induction Generator for Stirling Cogenerator System," in XIX International Conference on Electrical Machines, Rome, Italy, 1-7, 2010.

[60] T. Dang, M. Ruellan, H. Ben Ahmed, L. Prévond, and B. Multon, "Sizing Optimization of Tubular Linear Induction Generator for a New Stirling Micro-Cogenerator System," in International Symposium on Power Electronics, Electrical Drives, Automation and Motion, Ischia, Italy, 13621367, 2014.

[61] T. Dang, M. Ruellan, L. Prévond, H. Ben Ahmed, and B. Multon, "Sizing Optimization of Tubular Linear Induction Generator and Its Possible Application in High Acceleration Free-Piston Stirling Micro-Cogeneration," IEEE Transactions on Industry Applications, 51(5): 3716-3733, 2015.

[62] W. Cawthorne, "Optimization of a Brushless Permanent Magnet Linear Alternator for Use with a Linear Internal Combustion Engine," Ph.D. Dissertation, West Virginia University, Morgantown, WV, 1999.

[63] S. Mu, J. Chai, X. Sun, and S. Wang, "A Variable Pole Pitch Linear Induction Motor for Electromagnetic Aircraft Launch System," IEEE Transactions on Plasma Science, 43(5): 13461351, 2015.

[64] C. Wang and J. Shen, "A Method to Segregate Detent Force Components in Permanent-Magnet Flux-Switching Linear Machines," IEEE Transactions on Magnetics, 48(5): 1948-1955, 2012.

[65] F. Zhao, T. Asmus, D. Assanis, J. Dec, J. Eng, and P. Najt, Homogeneous Charge Compression Ignition ( $\mathrm{HCCl})$ Engines: Key Research and Development Issues, Warrendale, PA: SAE International, ISBN 978-0768011234, 1996.

[66] K. Motyl and T. Rychter, "HCCI Engine - A Preliminary Analysis," Journal of KONES Internal Combustion Engines, 10: 3-4, 2003. 
[67] J. Martinez-Frias, S. Aceves, D. Flowers, J. Smith, and R. Dibble, "HCCl Engine Control by Thermal Management," SAE Technical Paper 2000-01-2869, 2000.

[68] G. Haraldsson, P. Tunestål, B. Johansson, and J. Hyvönen, "HCCl Combustion Phasing in a Multi Cylinder Engine Using Variable Compression Ratio," SAE Technical Paper 2002-01-2858, 2002.

[69] G. Shaver and J. Gerdes, "Cycle-to-Cycle Control of HCCl Engines," in ASME International Mechanical Engineering Congress and Exposition, Washington, D.C., 2003.

[70] W. Polifke, W. Geng, and K. Dobbeling, "Optimization of Rate Coefficients for Simplified Reaction Mechanisms with Genetic Algorithms," Combustion and Flame, 113(1-2): 119-134, 1998.

[71] A. Poursamad and M. Montazeri, "Design of Genetic-Fuzzy Control Strategy for Parallel Hybrid Electric Vehicles," Control Engineering Practice, 16(7): 861-873, 2008.

[72] T. Sullivan, J. van de Ven, W. Northrop, and K. McCabe, "Integrated Mechanical and Thermodynamic Optimization of an Engine Linkage Using a Multi-Objective Genetic Algorithm," Journal of Mechanical Design, 137(2): 4, 2015.

[73] H. Aichlmayr and P. Van Blarigan, "Modeling and Experimental Characterization of a Permanent Magnet Linear Alternator for Free-Piston Engine Applications," in Proceedings of the $3^{\text {rd }}$ ASME International Conference on Energy Sustainability, San Francisco, CA, 761-770, 2009.

[74] P. Van Blarigan, S. Goldsborough, N. Paradiso, and J. Wu, "Homogeneous Charge Compression Ignition Free Piston Linear Alternator," SAND99-8206, 1998.

[75] J. Dec, "Advanced Compression-Ignition Engines - Understanding the In-Cylinder Processes," Proceedings of the Combustion Institute, 32: 2727-2742, 2009.

[76] T. de Paula Pignatti, W. Miziara, and R. de Cunha, "Reduced Friction for a Four Cylinder Two Valve Otto Engine Valve Train," SAE Technical Paper 2011-36-0208, 2011.

[77] M. Schwaderlapp, F. Koch, and J. Dohmen, "Friction Reduction - the Engine's Mechanical Contribution to Saving Fuel," SAE Technical Paper 2000-05-0160, 2000.

[78] C. Lee, "Turbine-Compound Free-Piston Linear Alternator Engine," Ph.D. Dissertation, University of Michigan, Ann Arbor, Ml, 2014.

[79] Q. Li, J. Xiao, and Z. Huang, "Simulation of a Two-Stroke Free-Piston Engine for Electrical Power Generation," Energy \& Fuels, 22(5): 3443-3449, 2008.

[80] G. Blair, Design and Simulation of Two-Stroke Engines, Warrendale, PA: SAE International, ISBN 978-1560916857, 1996.

[81] W. Cawthorne, P. Famouri, J. Chen, N. Clark, T. McDaniel, R. Atkinson, S. Nandkumar, C. Atkinson, and S. Petreanu, "Development of a Linear Alternator-Engine for Hybrid Electric Vehicle Applications," IEEE Transactions on Vehicular Technology, 48(6): 1797-1802, 1999. 
[82] P. Famouri, W. Cawthorne, N. Clark, S. Nandkumar, C. Atkinson, R. Atkinson, T. McDaniel, and S. Petreanu, "Design and Testing of a Novel Linear Alternator and Engine System for Remote Electrical Power Generation," in IEEE Power Engineering Society - 1999 Winter Meeting, New York, NY, 108-112, 1999.

[83] N. Hung and O. Lim, "A Study of a Two-Stroke Free Piston Linear Engine Using Numerical Analysis," Journal of Mechanical Science and Technology, 28(4): 1545-1557, 2014.

[84] Q. Li, J. Xiao, and Z. Huang, "Simulation of a Two-Stroke Free-Piston Engine for Electrical Power Generation," Energy \& Fuels, 22(5): 3443-3449, 2008.

[85] Q. Li, J. Xiao, and Z. Huang, "Parametric Study of a Free Piston Linear Alternator," International Journal of Automotive Technology, 11(1): 111-117, 2010.

[86] J. Chang, O. Güralp, Z. Filipi, D. Assanis, T. Kuo, P. Najt, and R. Rask, "New Heat Transfer Correlation for an $\mathrm{HCCl}$ Engine Derived from Measurements of Instantaneous Surface Heat Flux," SAE Technical Paper 2004-01-2996, 2004.

[87] G. Hohenberg, "Advanced Approaches for Heat Transfer Calculations," SAE Technical Paper 790825, 1979.

[88] T. Bancha and J. Lu, "A Single Zone Model with Double-Wiebe Function for the Prediction of nHeptane Fuelled $\mathrm{HCCl}$ Engine Performance," Journal of the Chinese Society of Mechanical Engineers, 34(1): 1-9, 2013.

[89] J. Kim, C. Bae, and G. Kim, "Simulation on the Effect of the Combustion Parameters on the Piston Dynamics and Engine Performance Using the Wiebe Function in a Free Piston Engine," Applied Energy, 107: 446-455, 2013.

[90] J. Kodavasal, C. Kolodziej, S. Ciatti, and S. Som, "Computational Fluid Dynamics Simulation of Gasoline Compression Ignition," ASME Journal of Energy Resources Technology, 137(3): 13, 2015.

[91] J. Kodavasal, G. Lavoie, D. Assanis, and J. Martz, "The Effect of Diluent Composition on Homogeneous Charge Compression Ignition Auto-Ignition and Combustion Duration," Proceedings of the Combustion Institute, 35: 3019-3026, 2015.

[92] Y. Mo, "HCCl Heat Release Rate and Combustion Efficiency: A Coupled KIVA Multi-Zone Modeling Study," Ph.D. Dissertation, University of Michigan, Ann Arbor, MI, 2008.

[93] J. Ghojel, "Review of the Development and Applications of the Wiebe Function: A Tribute to the Contribution of Ivan Wiebe to Engine Research," International Journal of Engine Research, 11(4): 297-312, 2010. 
[94] J. Godino, M. Garcia, F. Aguilar, and E. Trujillo, "Numerical Study of HCCl Combustion Fueled with Diesel Oil Using A Multizone Model Approach," Energy Conversion and Management, 89: 885-895, 2015.

[95] R. Maurya and A. Agarwal, "Experimental Investigations of Performance, Combustion and Emission Characteristics of Ethanol and Methanol Fueled $\mathrm{HCCl}$ Engine," Fuel Processing Technology, 126: 30-48, 2014.

[96] R. Maurya and A. Agarwal, "Combustion and Emission Characterization of n-Butanol Fueled HCCI Engine," ASME Journal of Energy Resources Technology, 137(1): 12, 2015.

[97] K. Swan, M. Shahbakhti, and C. Koch, "Predicting Start of Combustion Using a Modified Knock Integral Method for an HCCI Engine," SAE Technical Paper 2006-01-1086, 2006.

[98] X. He, M. Donovan, B. Zigler, T. Palmer, S. Walton, M. Wooldridge, and A. Atreya, "An Experimental and Modeling Study of iso-Octane Ignition Delay Times Under Homogeneous Charge Compression Ignition Conditions," Combustion and Flame, 142(3): 266-275, 2005.

[99] G. Woschni, "A Universally Applicable Equation for the Instantaneous Heat Transfer Coefficient in the Internal Combustion Engine," SAE Technical Paper 670931, 1967.

[100] W. Annand, "Heat Transfer in the Cylinders of Reciprocating Internal Combustion Engines," Proceedings of the Institution of Mechanical Engineers, 177(1): 973-996, 1963.

[101] S. Broekaert, T. de Cuyper, K. Chana, M. de Paepe, and S. Verhelst, "Assessment of Empirical Heat Transfer Models for a CFR Engine Operated in HCCI Mode," SAE Technical Paper 201501-1750, 2015.

[102] H. Yasar, H. Soyhan, H. Walmsley, B. Head, and C. Sorusbay, "Double-Wiebe Function: An approach for Single-Zone HCCl Engine Modeling," Applied Thermal Engineering, 28(11-12): 1284-1290, 2008.

[103] E. Shoukry, "Numerical Simulation for Parametric Study of a Two-Stroke Compression Ignition Direct Injection Linear Engine," Ph.D. Dissertation, West Virginia University, Morgantown, WV, 2003.

[104] A. Eid, K. Suh, K. Choi, H. Han, H. Lee, and M. Nakaoka, "A Unique Starting Scheme of LinearEngine Tubular PM Linear Generator System Using Position Feedback Controlled PWM Inverter," in 37 ${ }^{\text {th }}$ IEEE Power Electronics Specialist Conference, Cheju Isle, South Korea, 1308-1312, 2006. 


\section{APPENDIX A - ADDITIONAL SIMULINK MODEL DIAGRAMS}

This appendix provides additional figures for the Simulink modeling subsystems which are not included in the main text body.

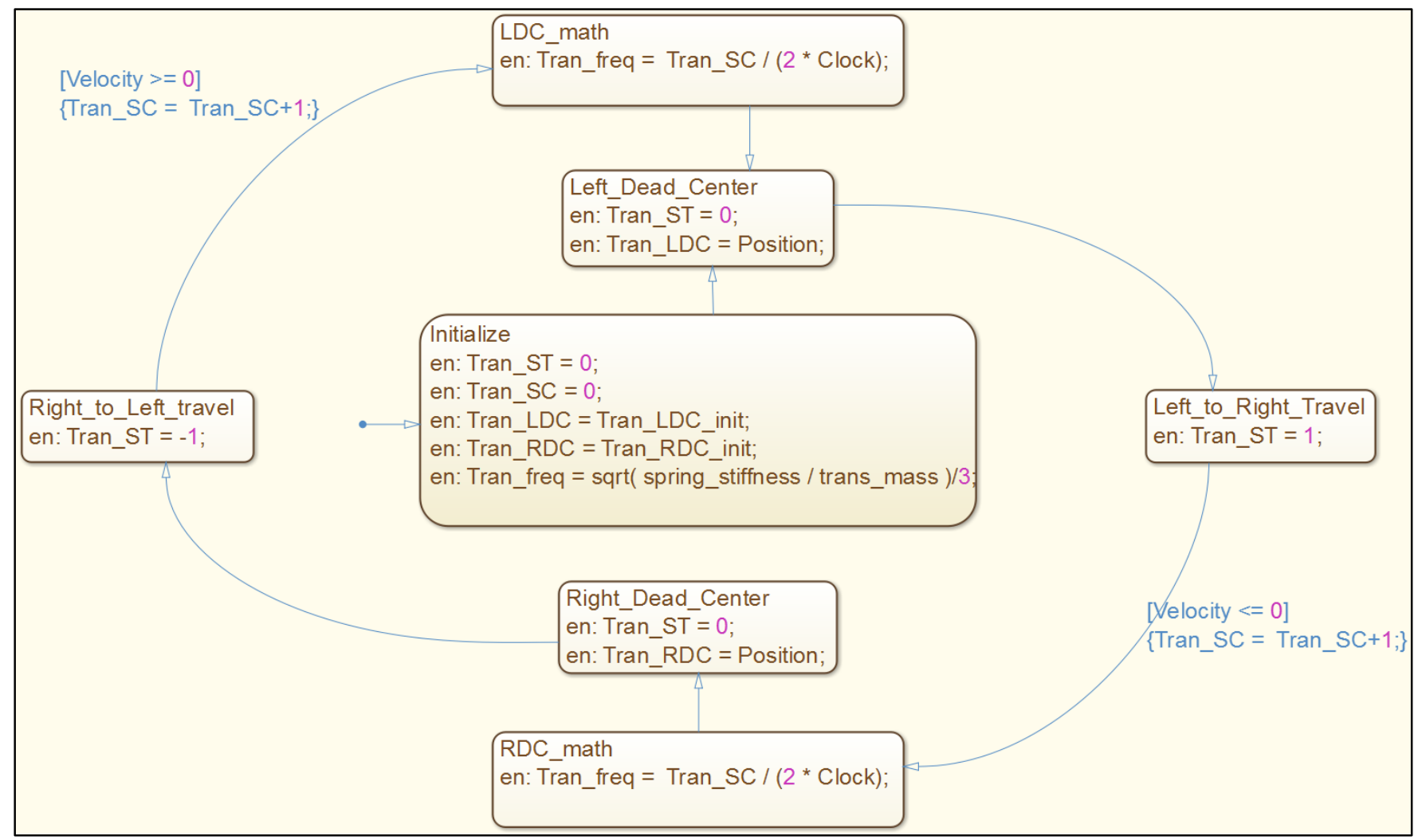

Figure 109: 'Translator State' subsystem of the Simulink model.

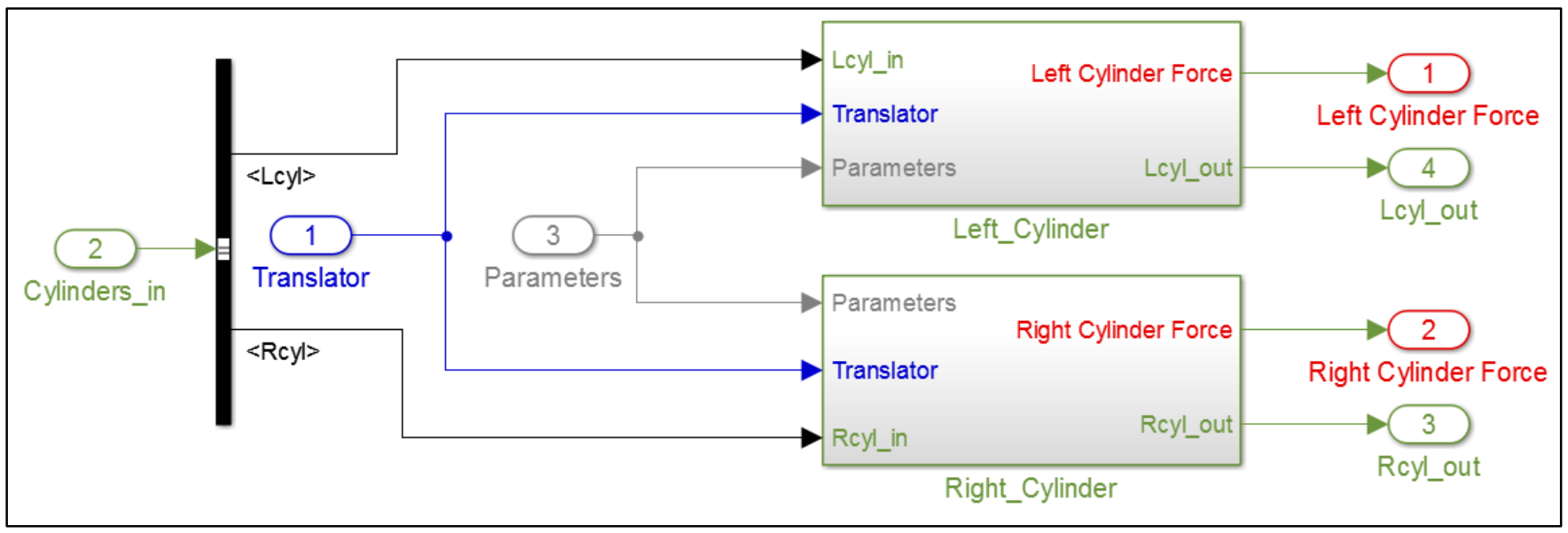

Figure 110: ‘Cylinder Pressures' subsystem of the Simulink model. 


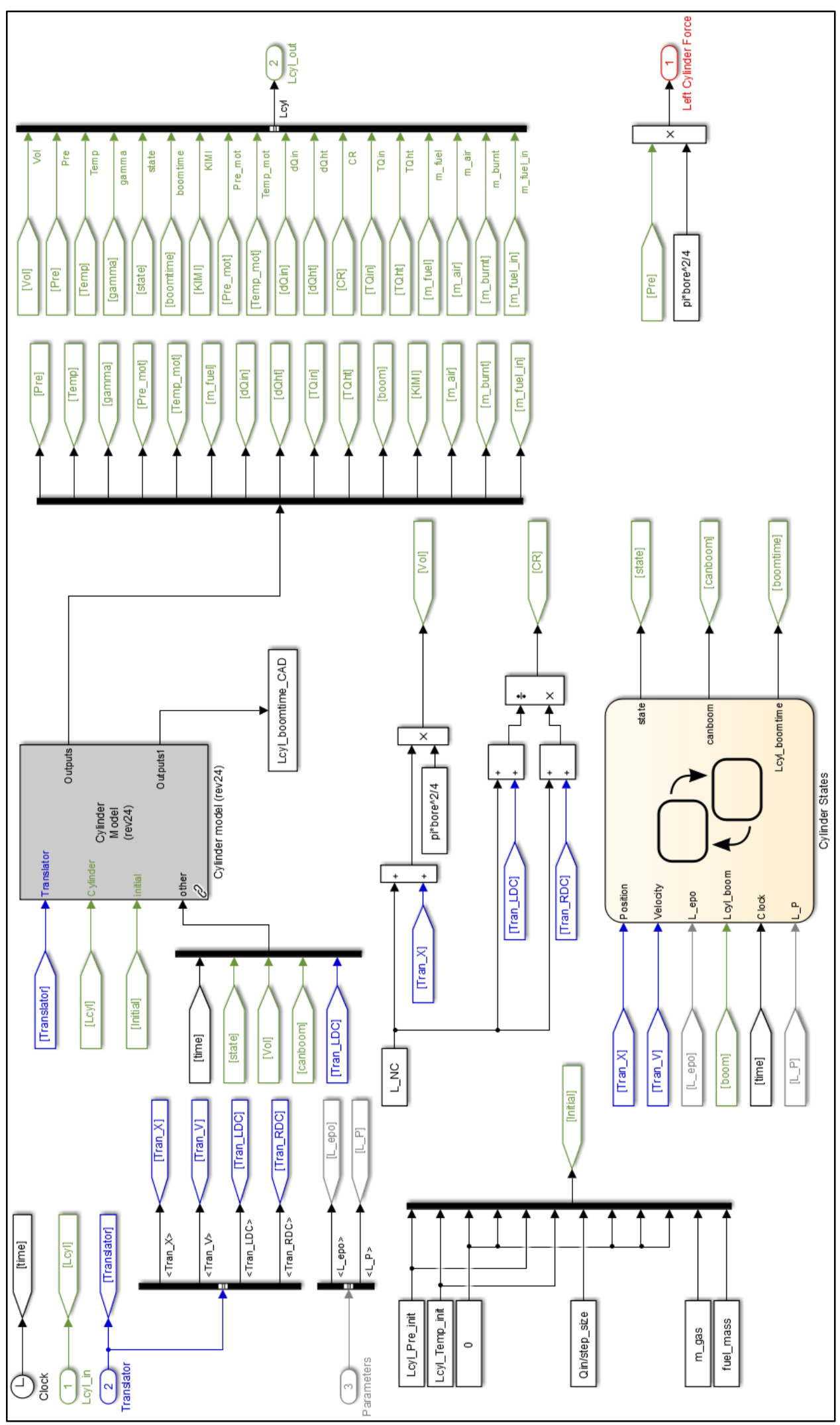

Figure 111: 'Left_Cylinder' subsystem of the Simulink model. 


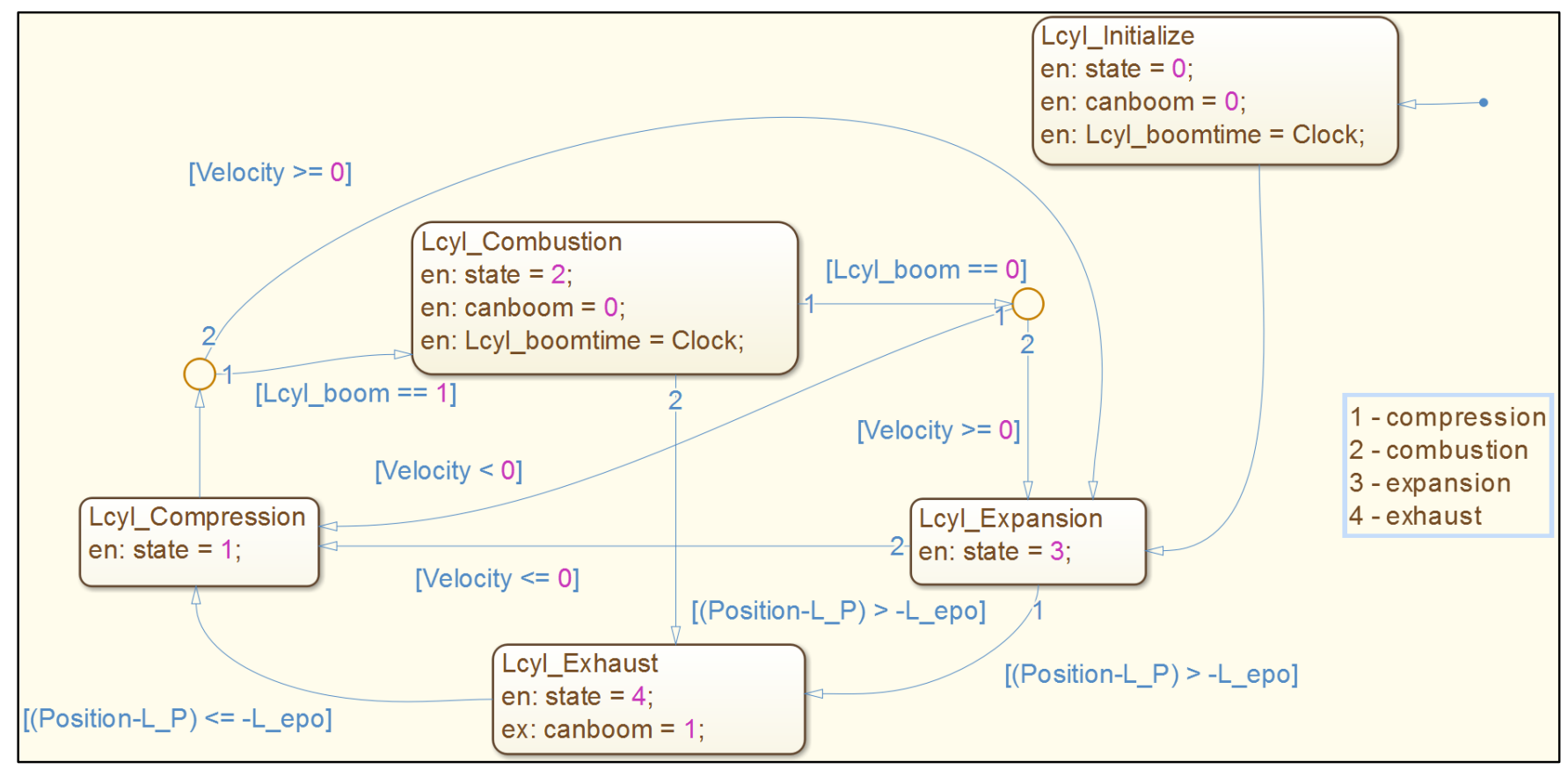

Figure 112: 'Cylinder States' subsystem of the Simulink model.

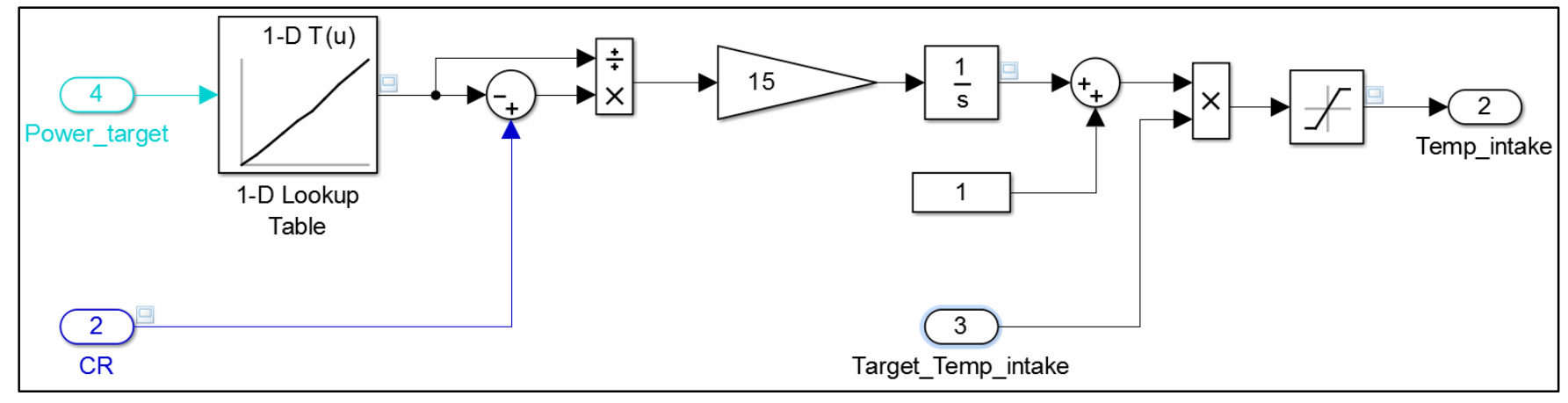

Figure 113: 'Intake control' subsystem of the Simulink model.

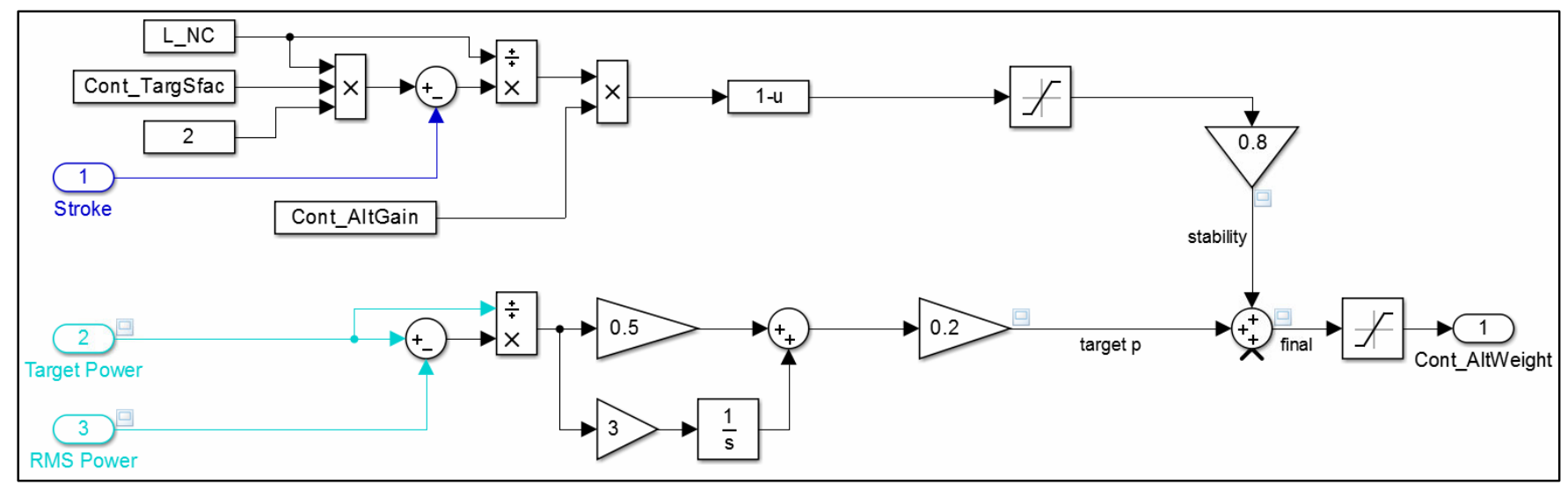

Figure 114: 'Alternator control' subsystem of the Simulink model. 


\section{APPENDIX B - MATLAB ${ }^{\circledR}$ SCRIPTS AND FUNCTIONS}

\section{VIII (i) Parametric Study}

$M A T L A B^{\circledR}$ script used to compile the Simulink model and perform the various parametric sweeps.

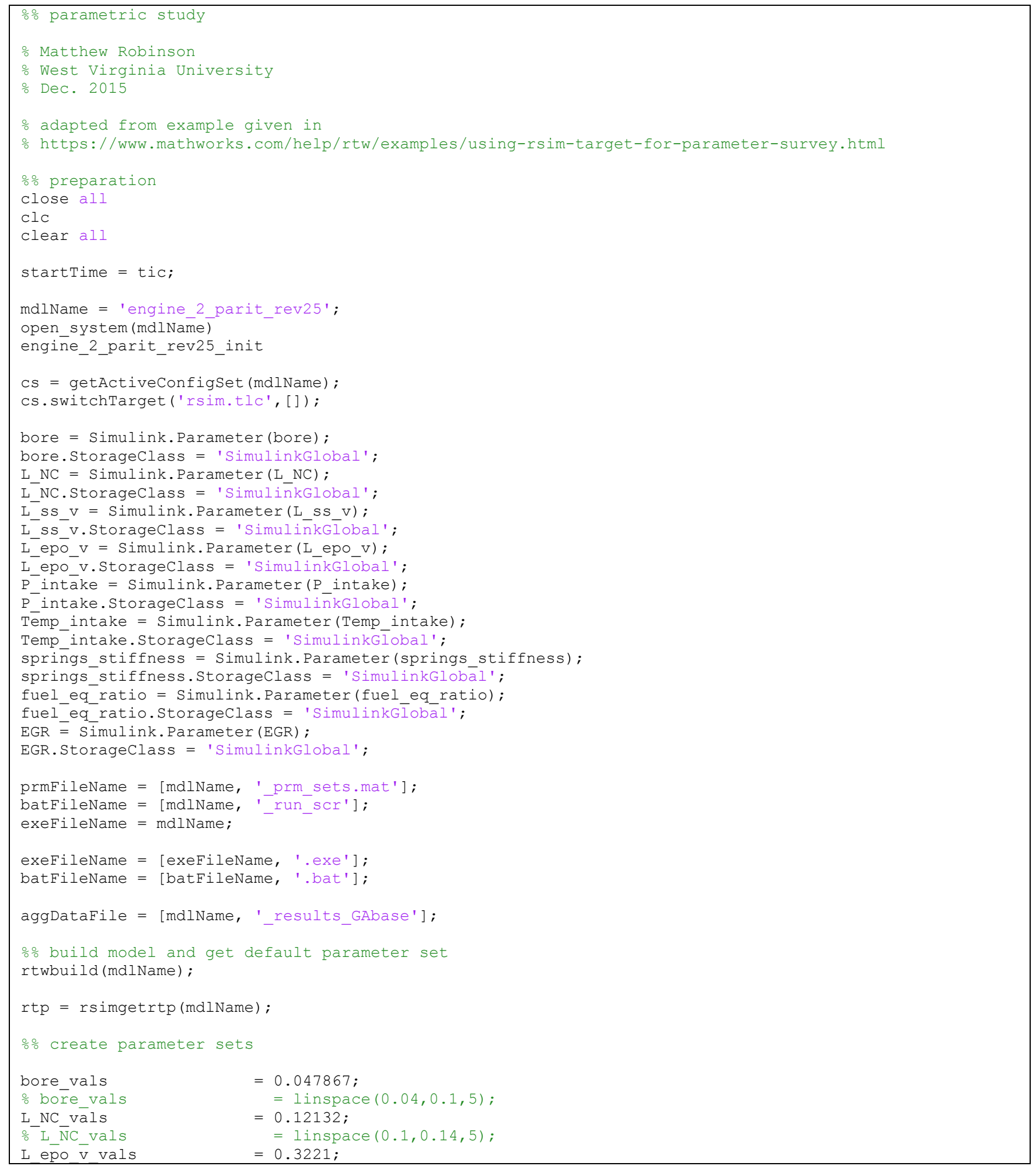




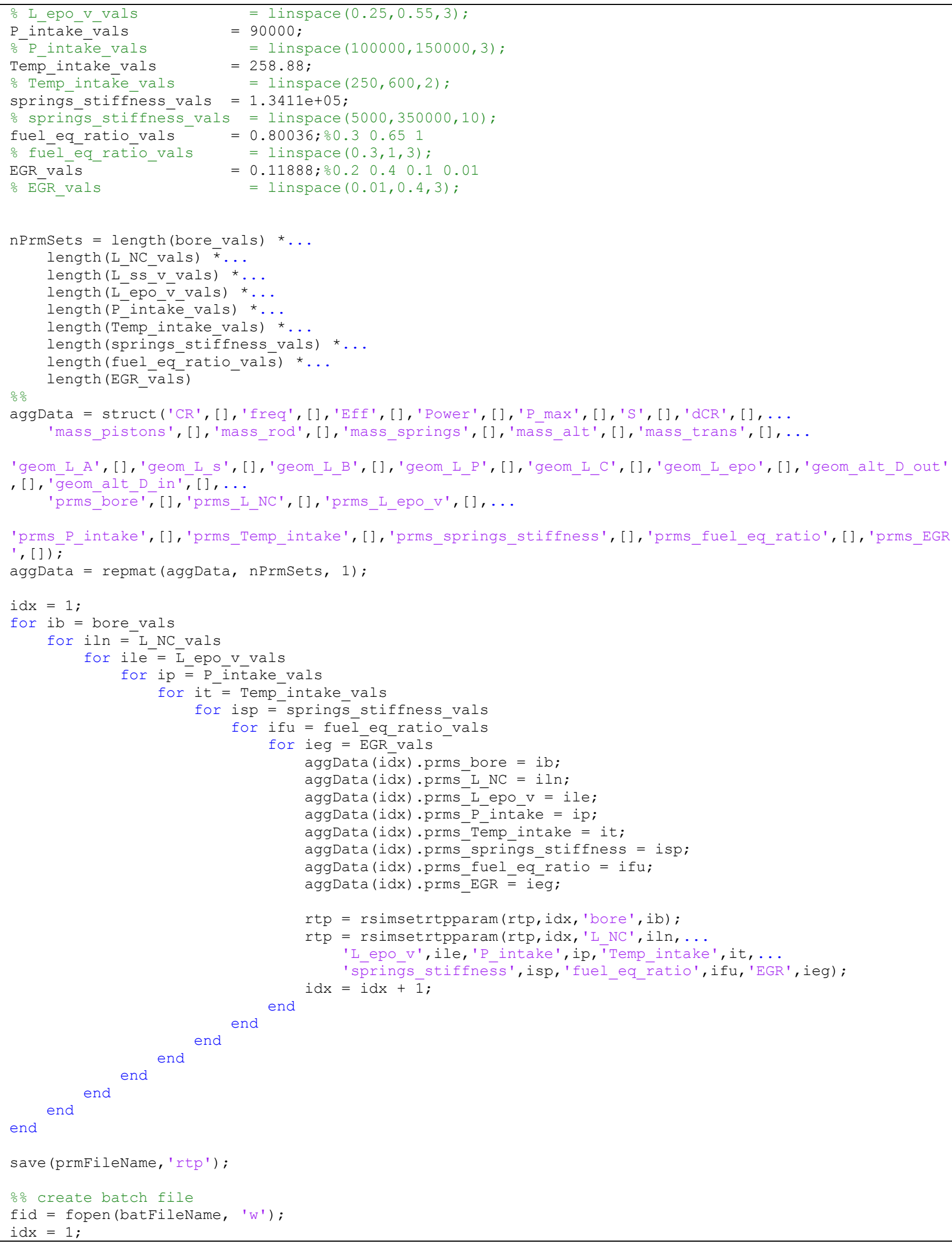




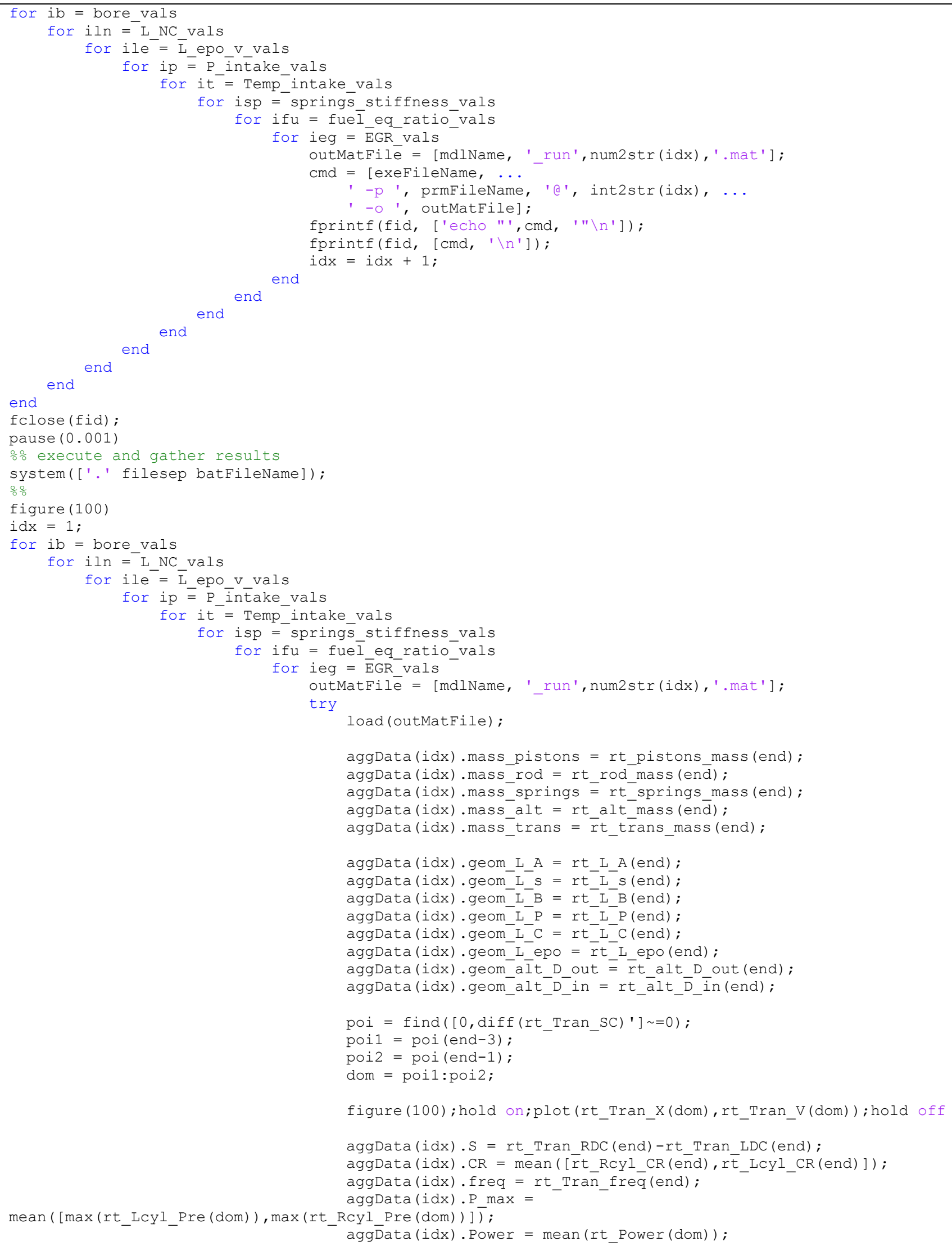




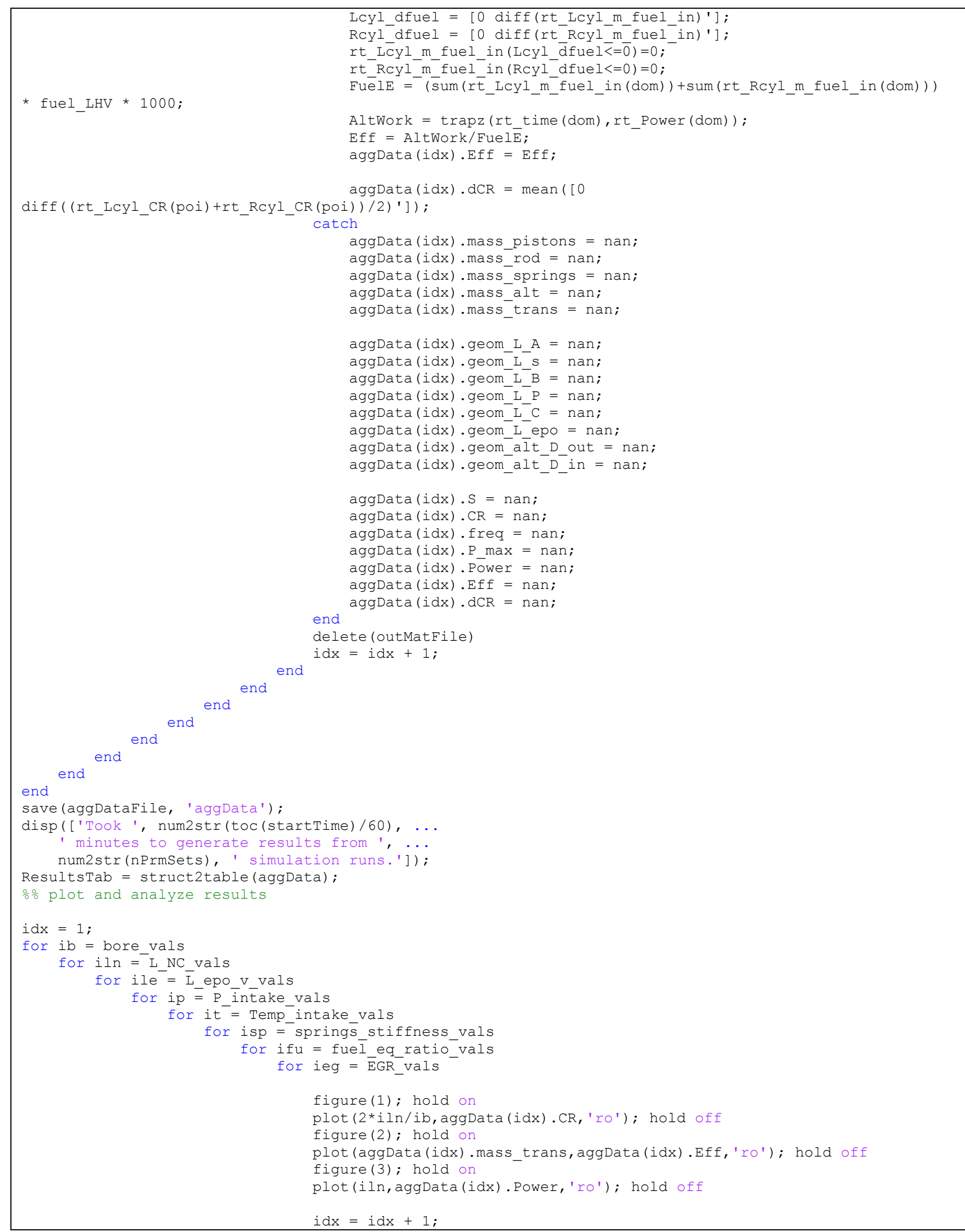




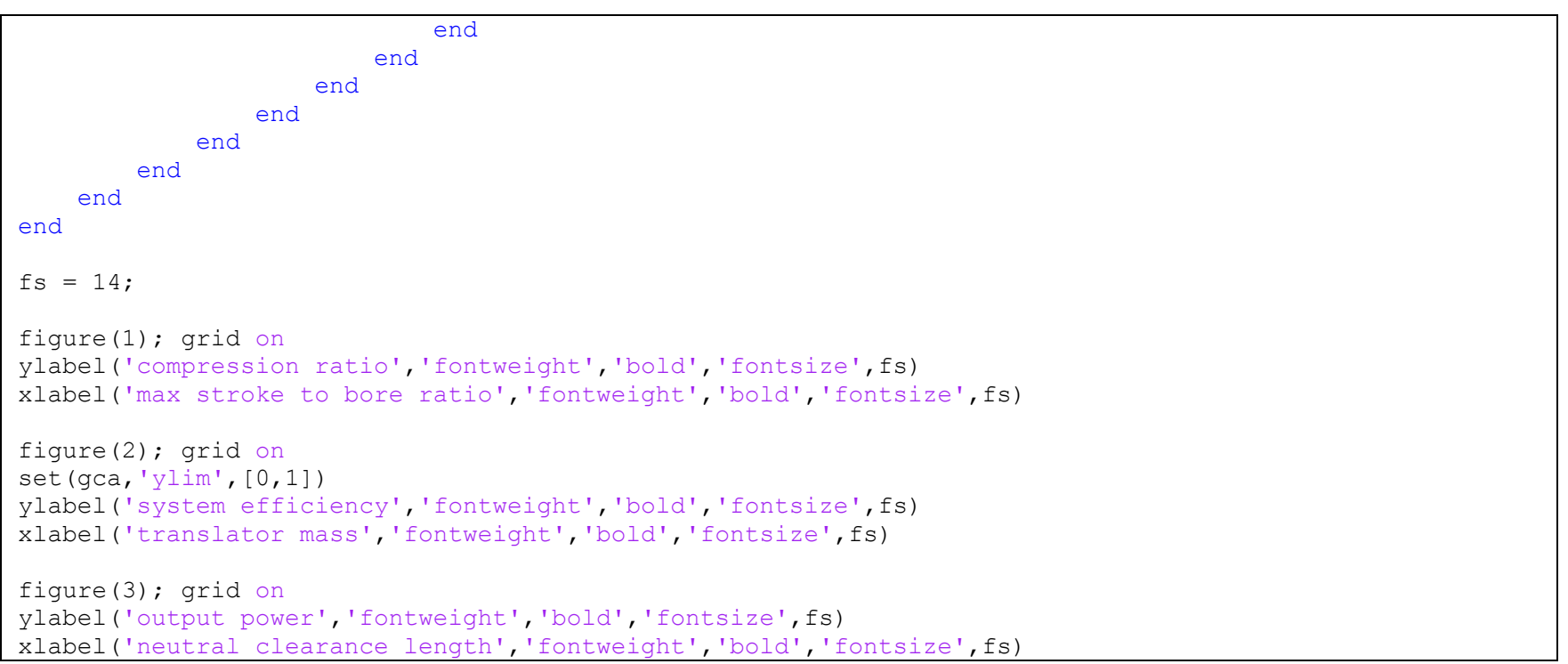

\section{VIII (ii) Genetic Algorithm}

This is the master script which calls the initial population and allows the user to set optimization parameters.

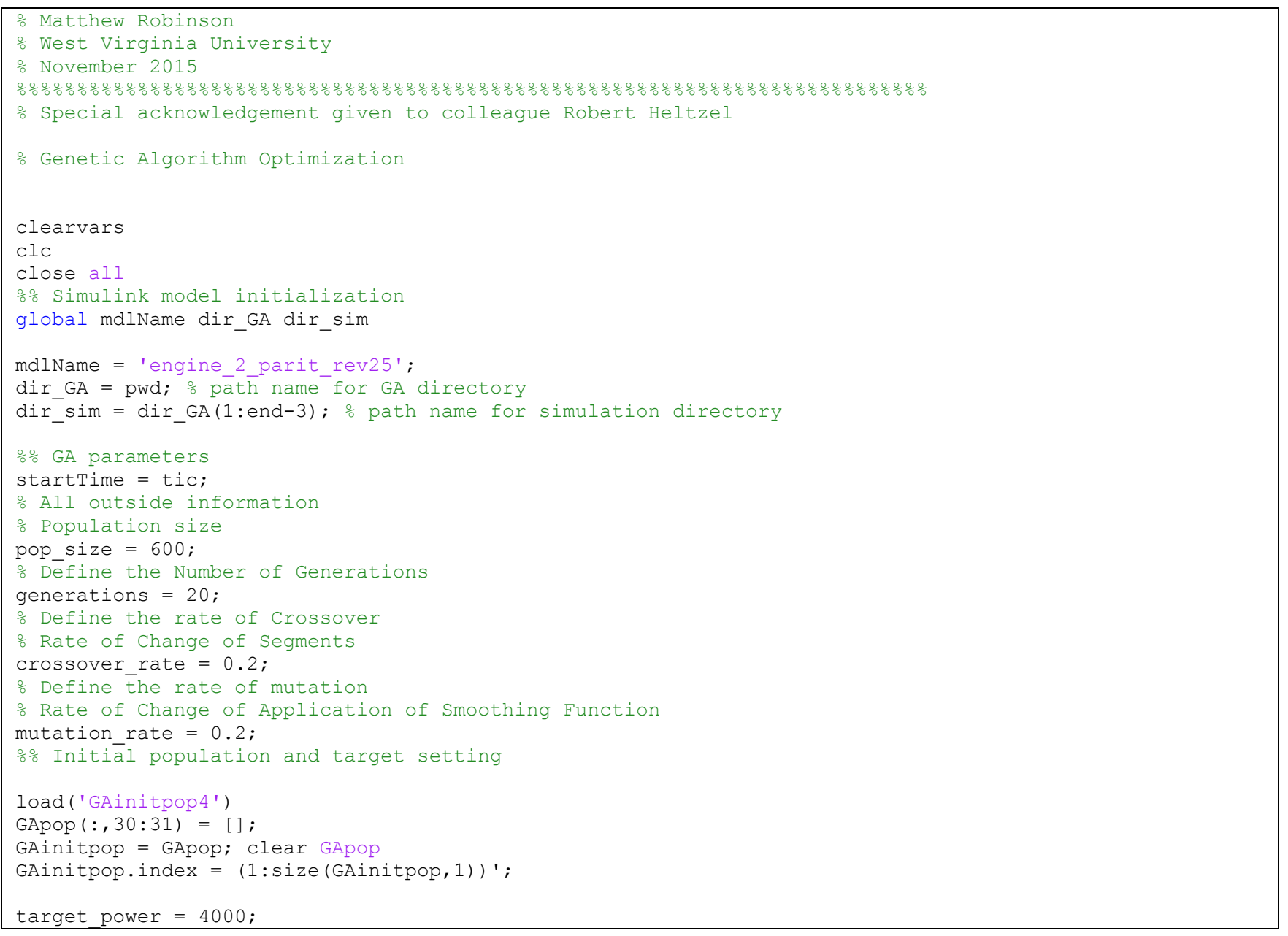




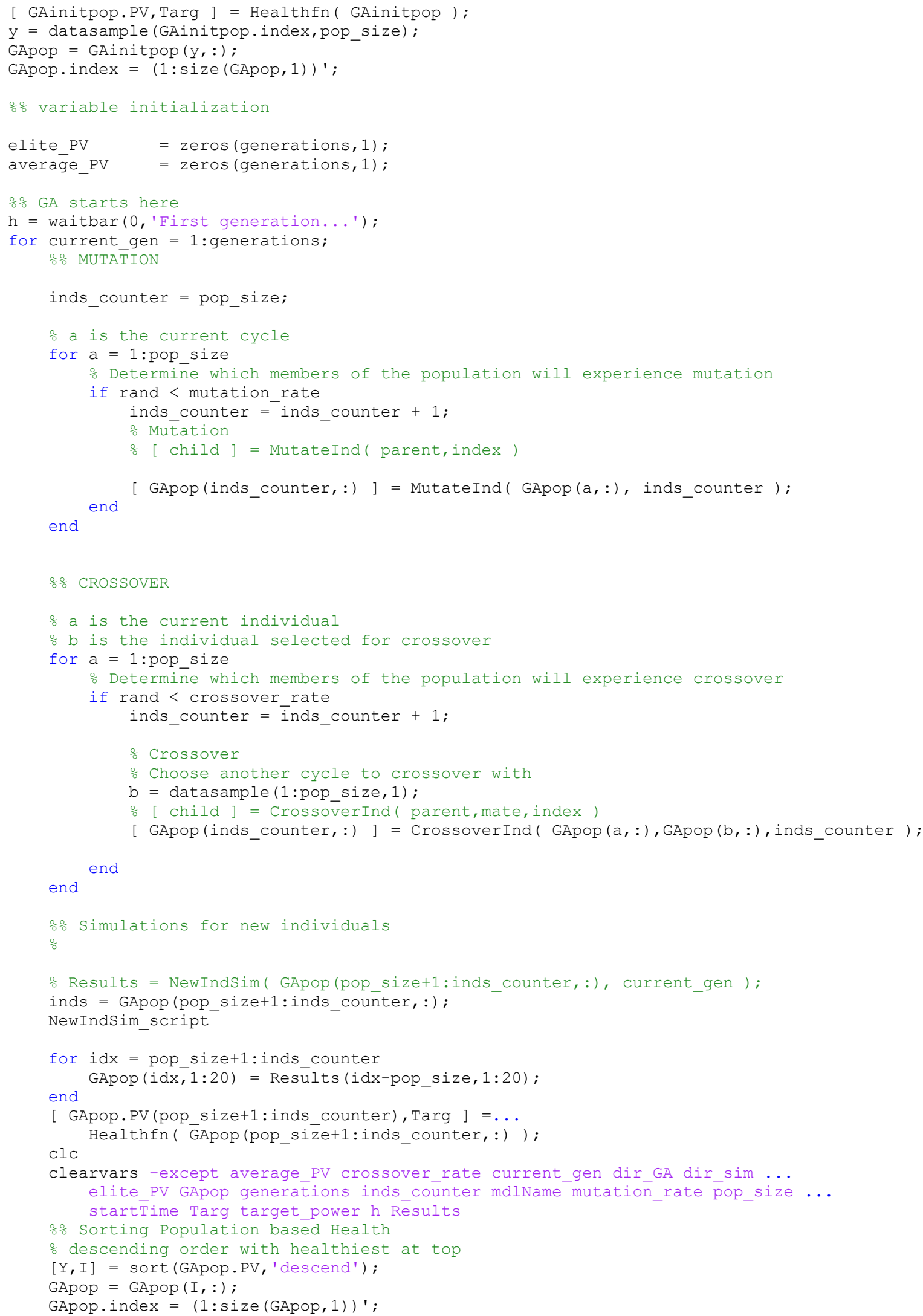




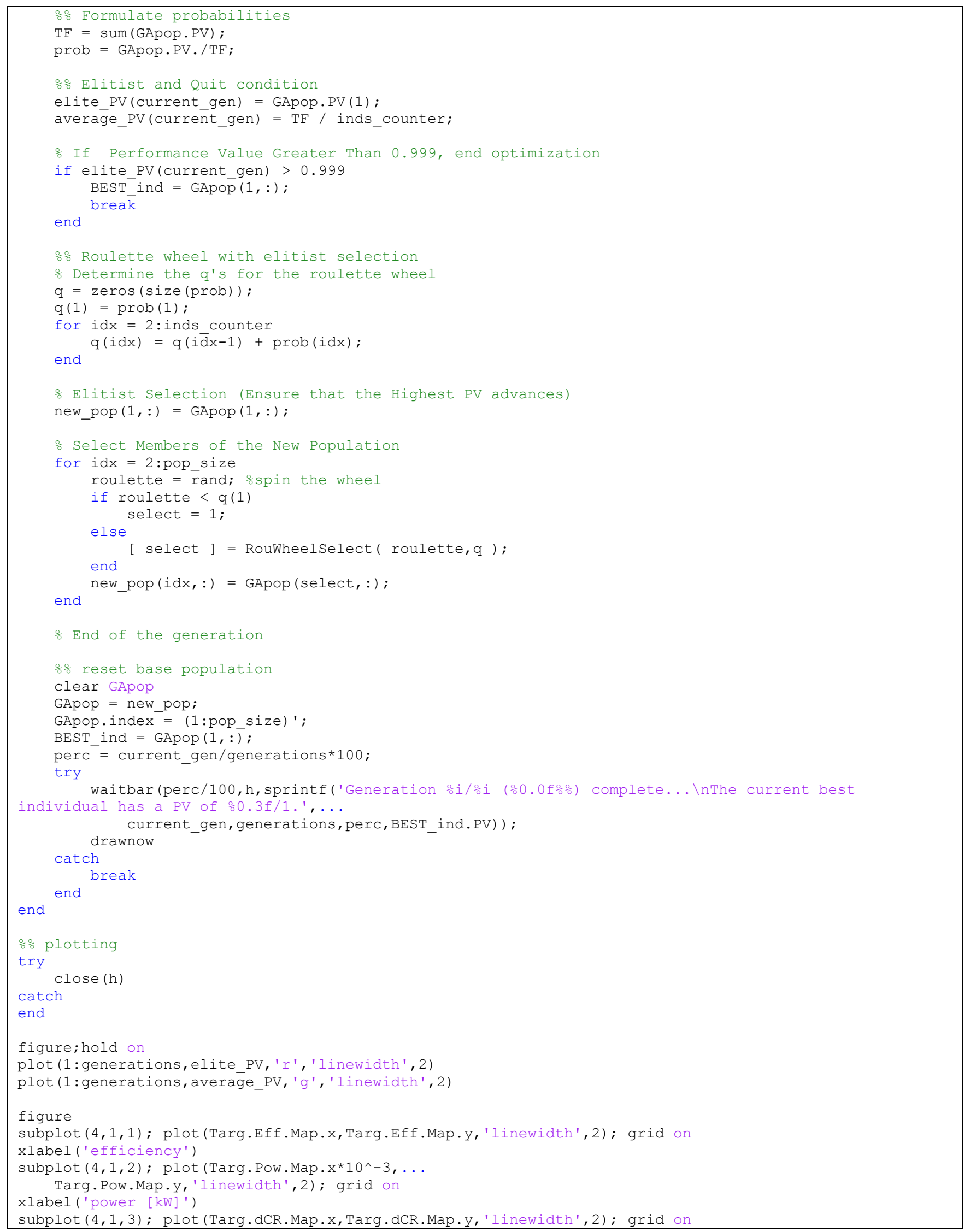


xlabel ('stability [dCR/dt]')

subplot $(4,1,4)$; plot (Targ.P_max.Map. $x^{\star} 10^{\wedge}-5, \ldots$

Targ.P max.Map.y, 'linewidth',2); grid on

xlabel ('max pressure [bar]')

\section{The health or performance value of each individual is assessed with this function.}

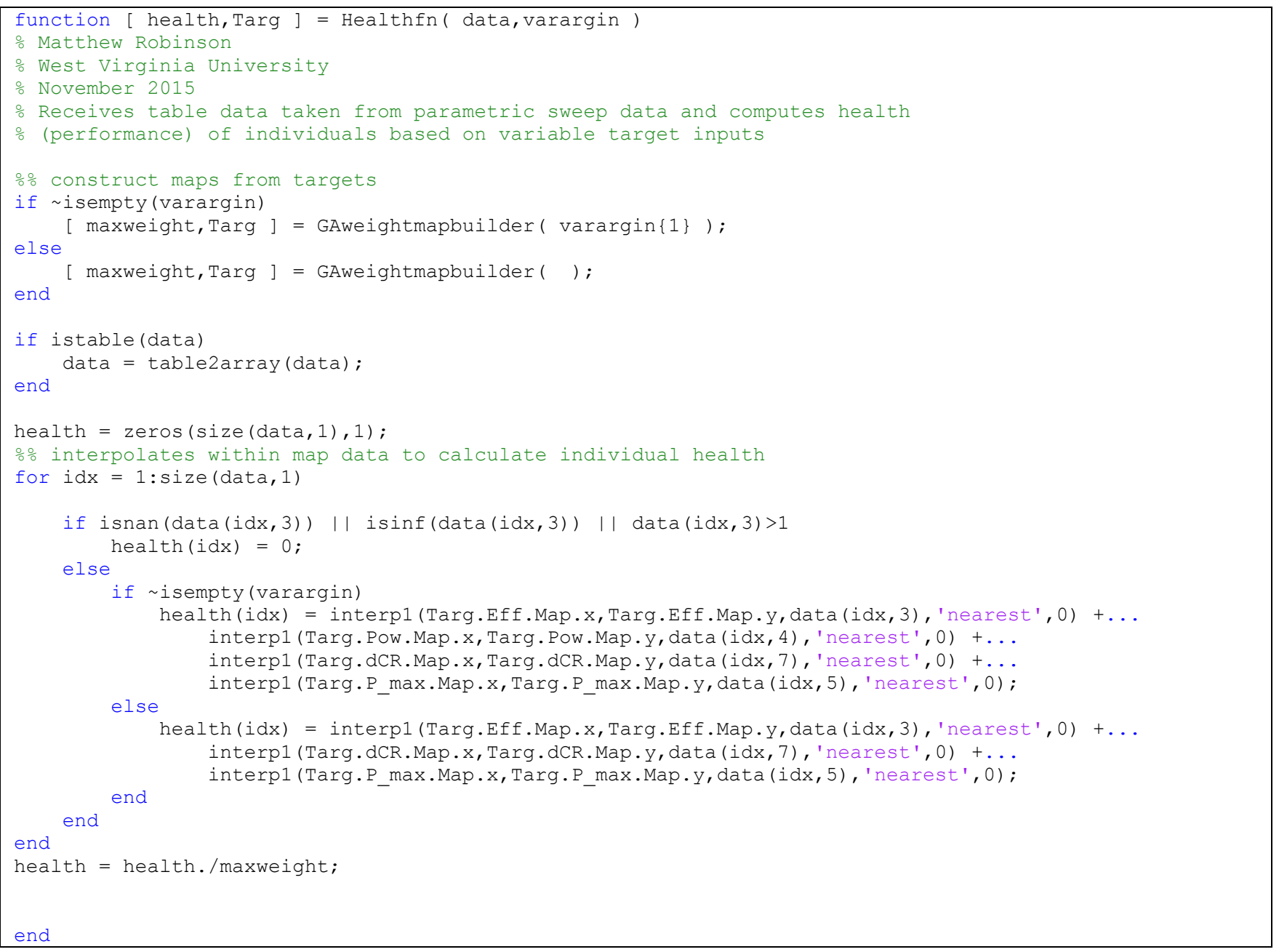

The performance target weight maps are built by the following function which receives variable inputs based on user specification.

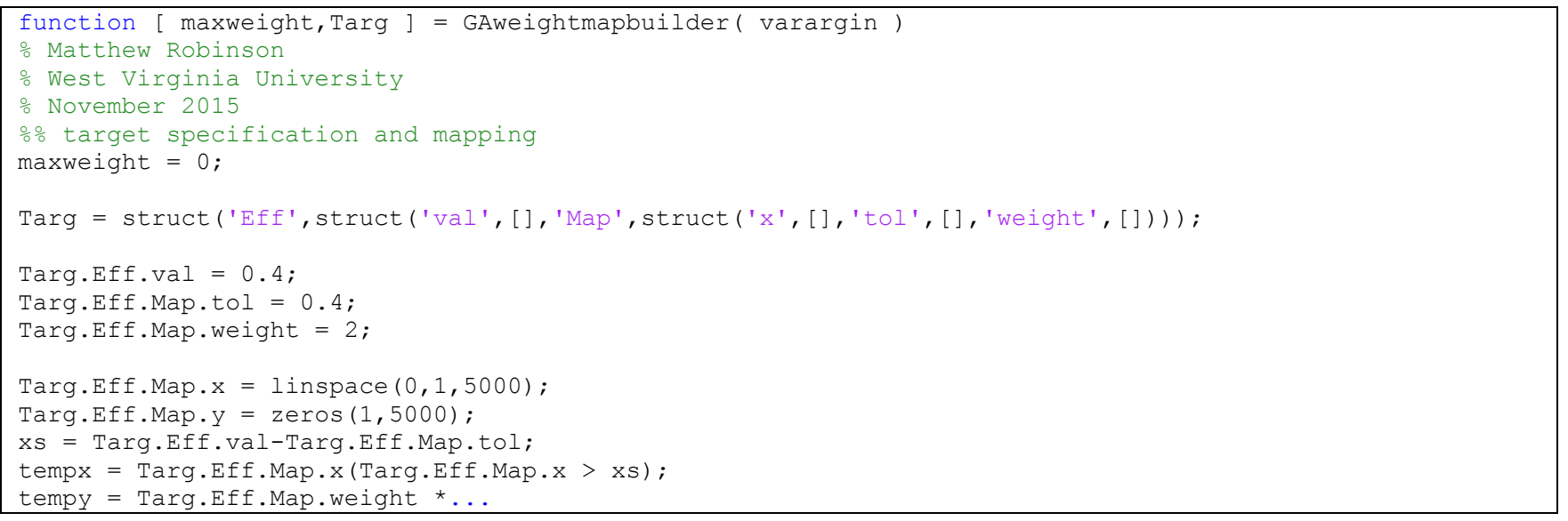




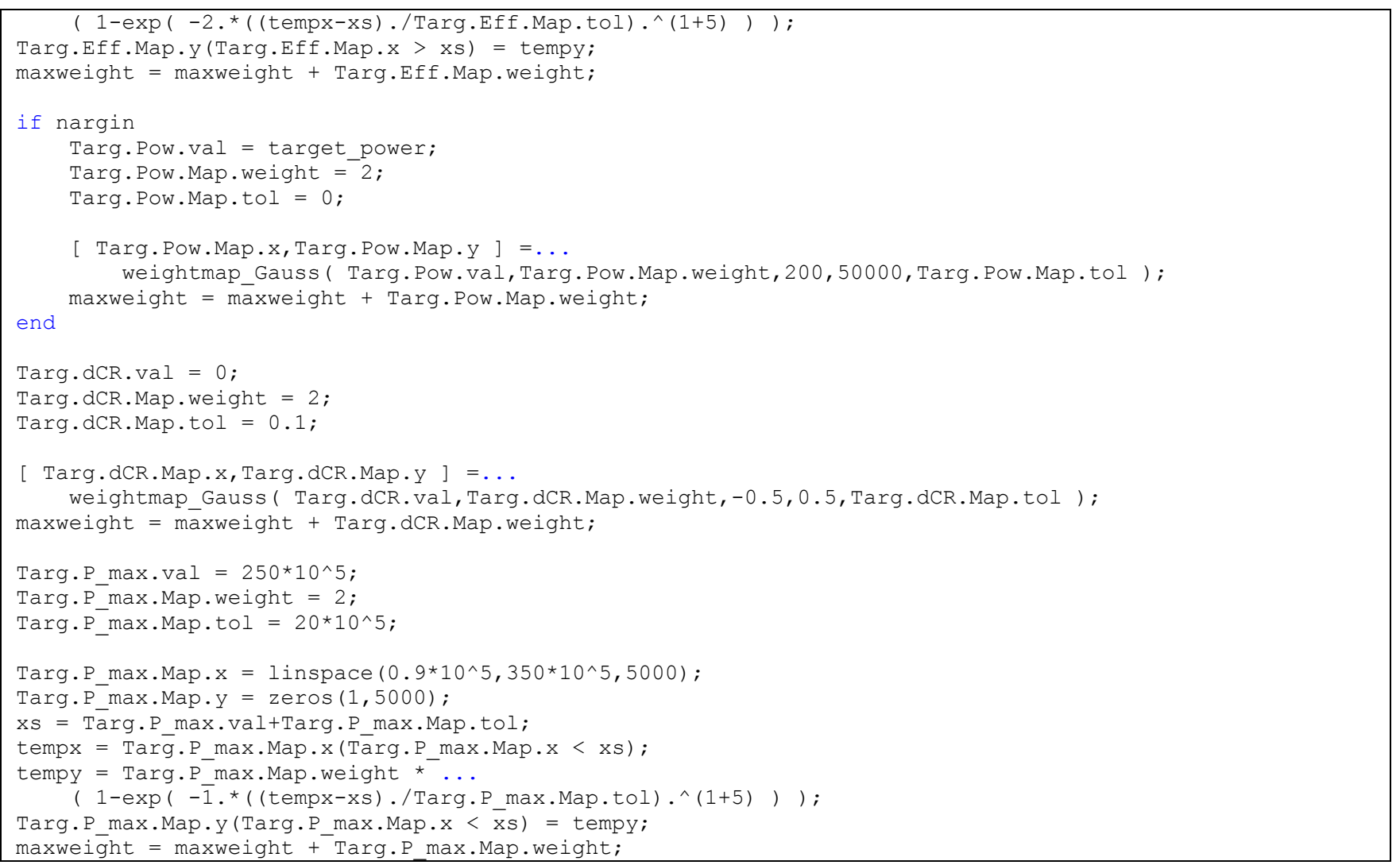

Individuals undergo mutation according to the mutation rate and this function.

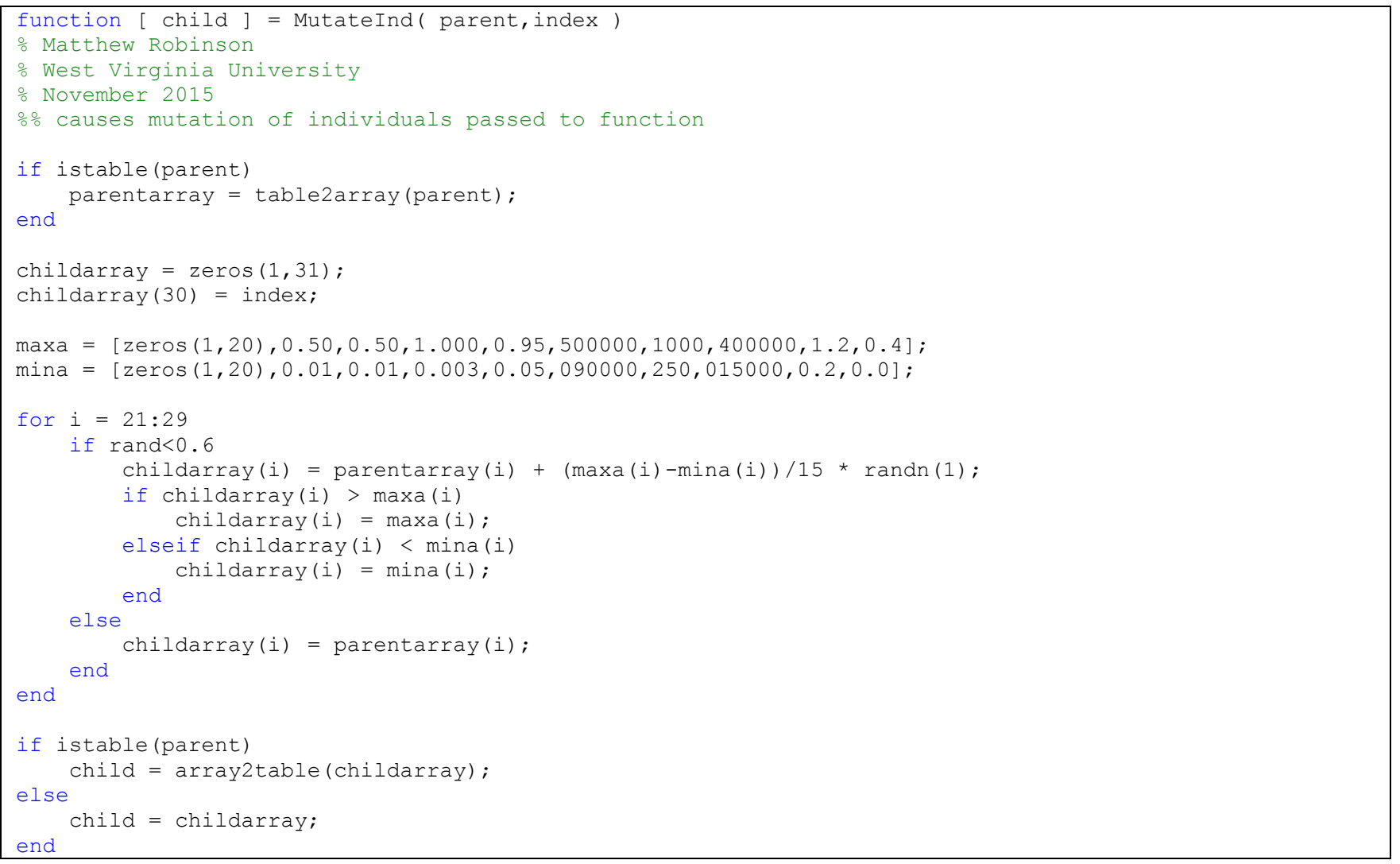




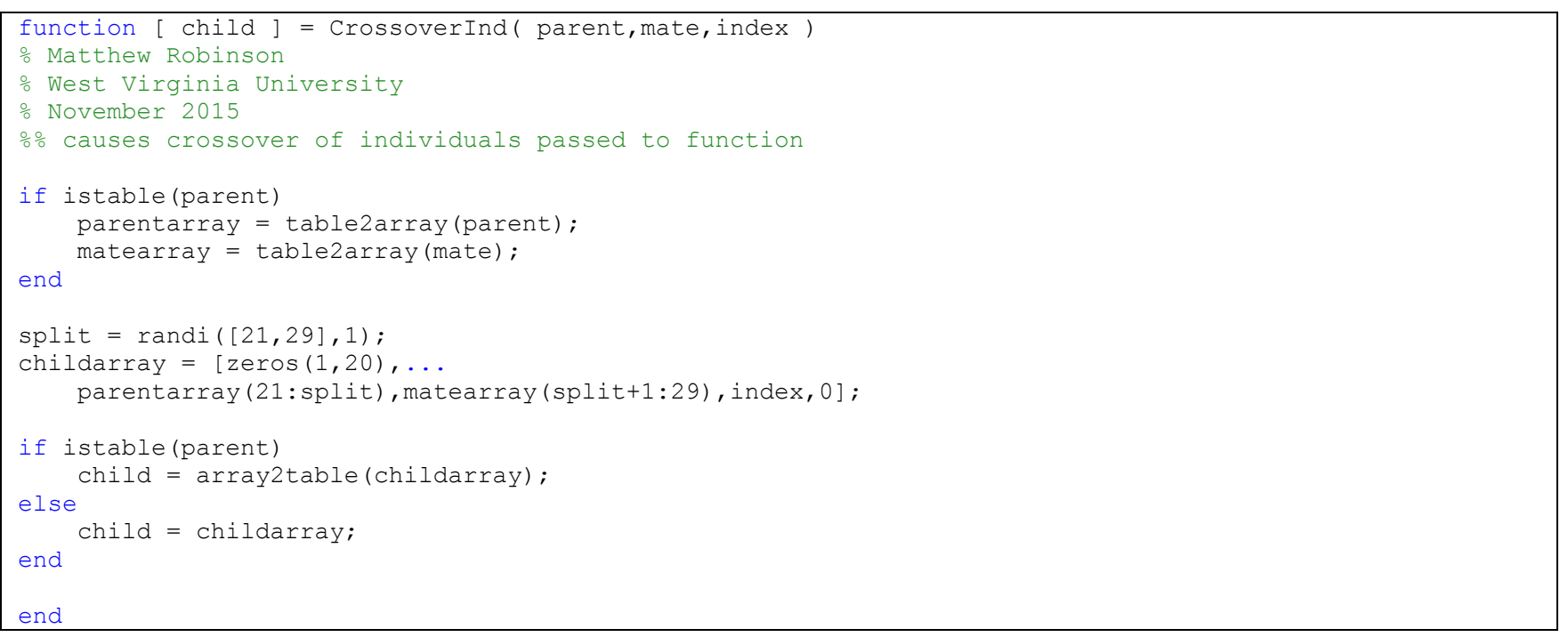

New parameter sets developed by mutation and crossover are simulated by with the use of a script similar to the parameter sweep script.

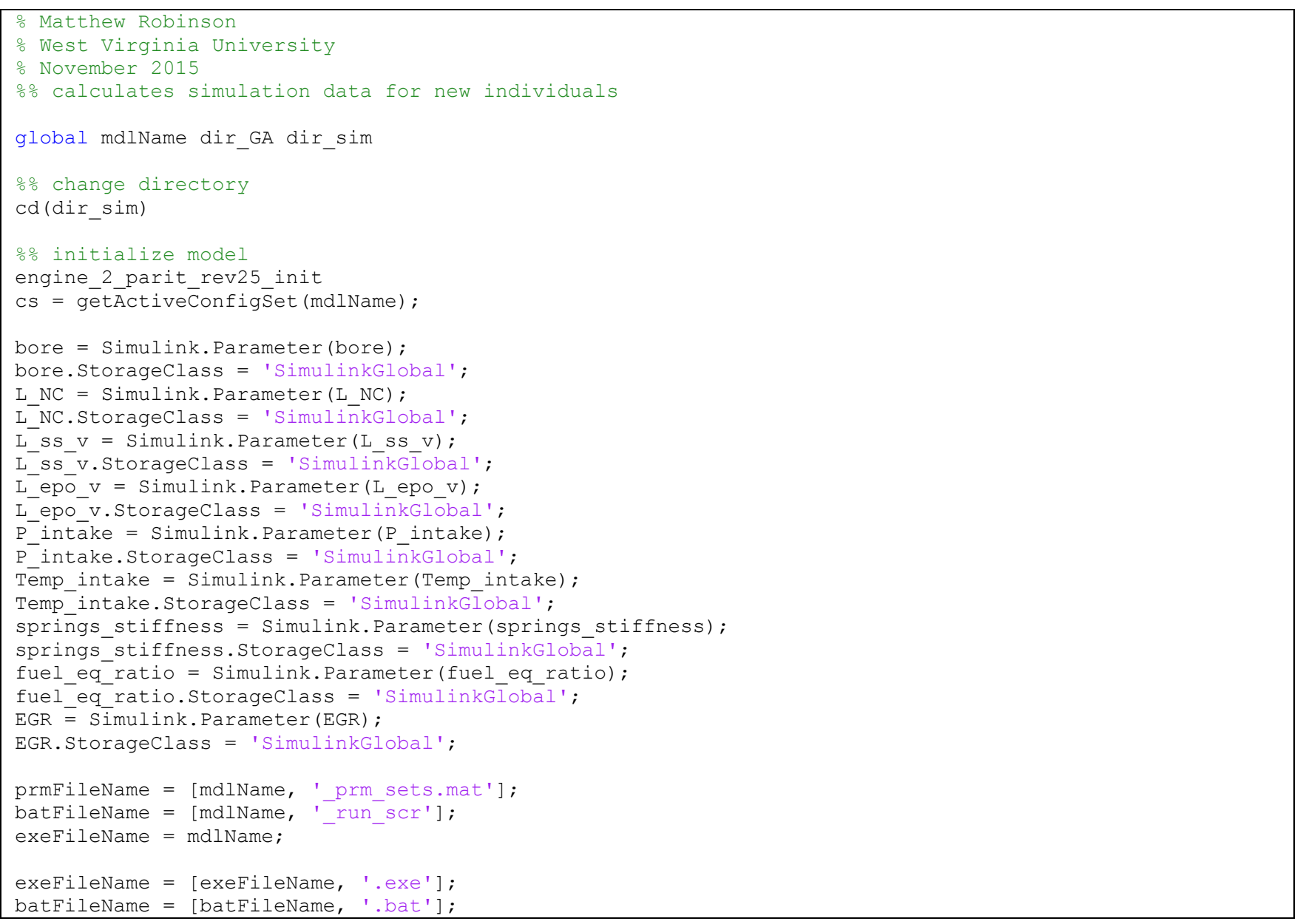




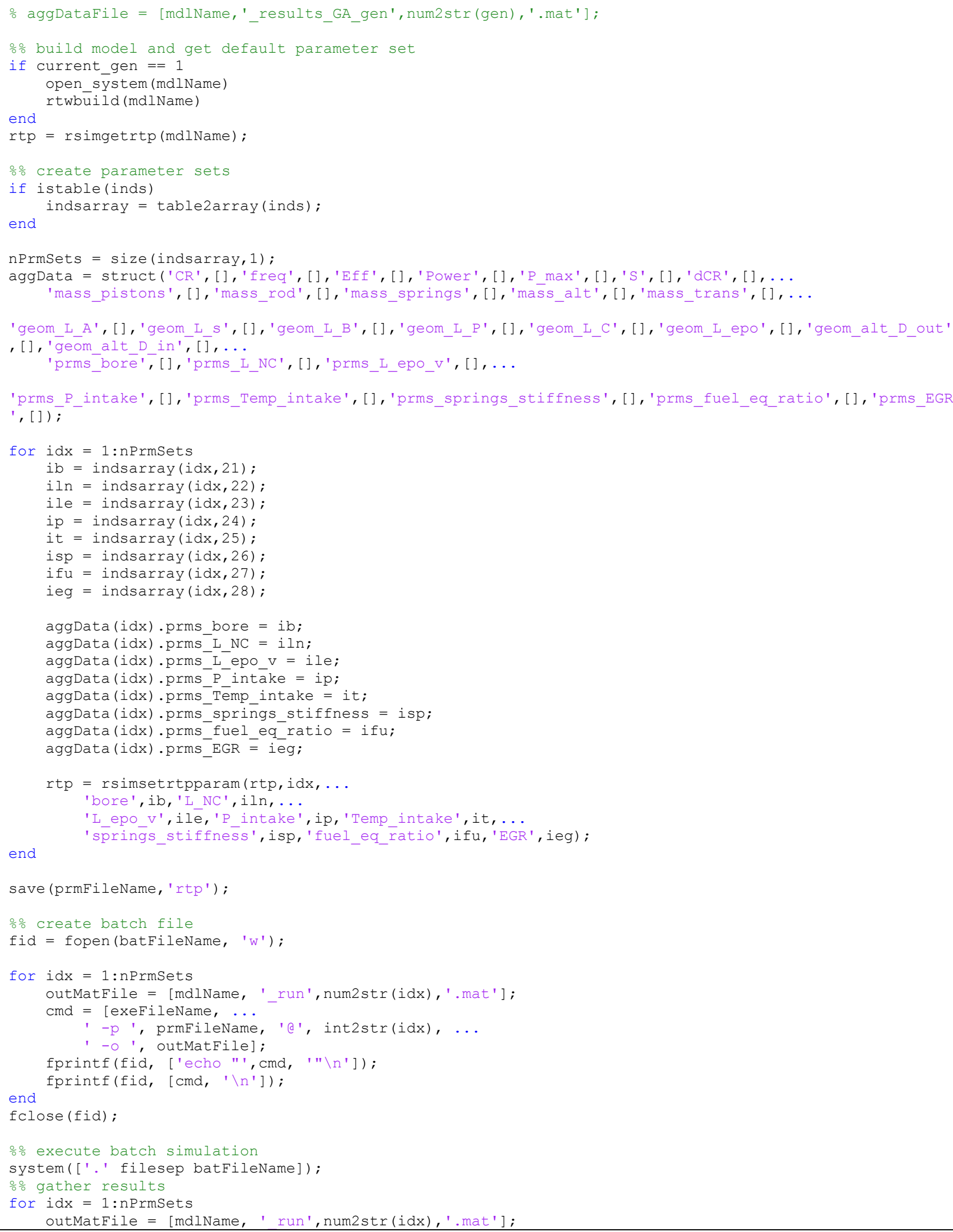




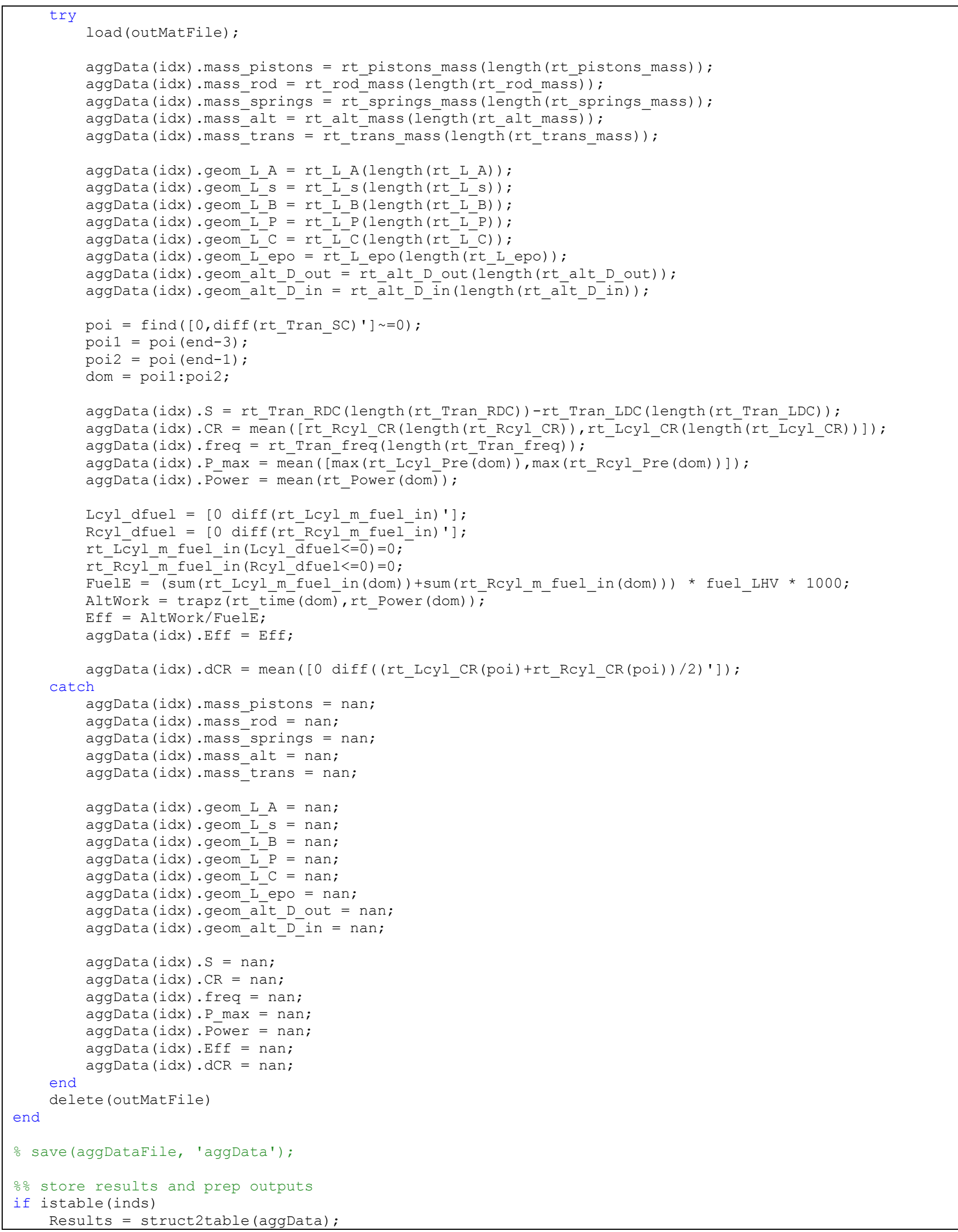




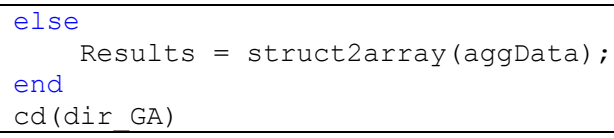

After performance of new individuals has been reassessed by the health function, roulette wheel selection is used to form the new population.

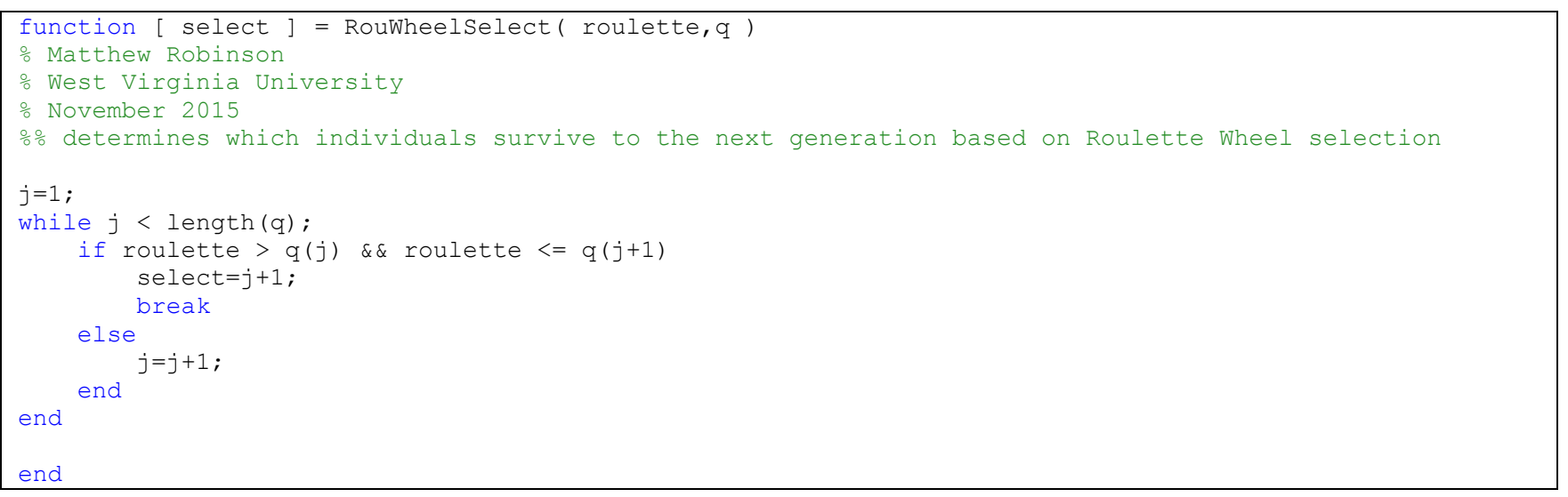




\section{APPENDIX C - SUPPLEMENTAL FIGURES FROM PARAMETRIC STUDY}
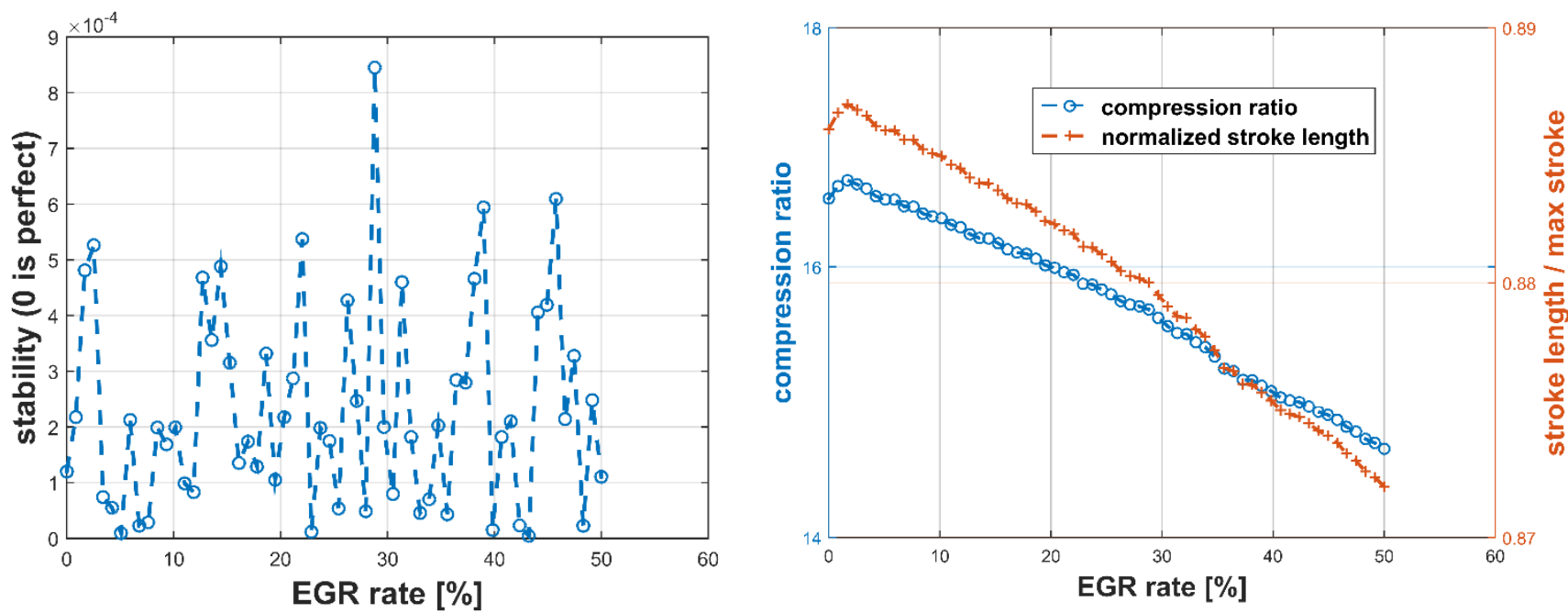

Figure 115: Effects of EGR on stability, compression ratio, and stroke length for the testing and validation base case with the RCM ignition delay model.
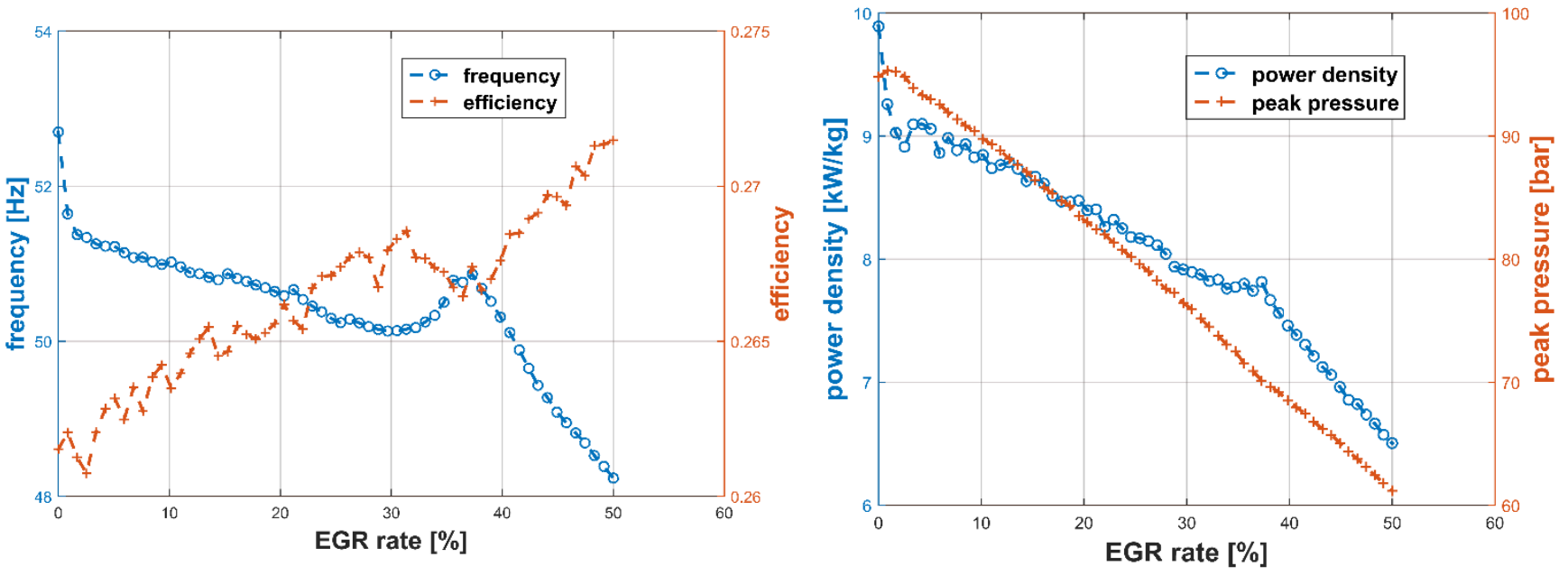

Figure 116: Effects of EGR on frequency, efficiency, power density, and peak pressure for the testing and validation base case with RCM ignition delay model

The following figures (Figure 117 - Figure 124) present parametric study data in support of Ch. 4. They are presented as functions of four dimensionless parameters:

1. maximum stroke to bore ratio

$$
\frac{S_{\max }}{b}
$$

2. dimensionless intake pressure (stemming from the fundamental analysis)

$$
\frac{\pi b^{2} P_{\text {in }}}{4 L k}
$$

3. dimensionless heat addition (stemming from the fundamental analysis)| 


$$
\frac{Q_{i n}}{k L^{2}}
$$

4. actual steady state to fundamental frequency ratio

$$
\frac{f_{s s}}{\sqrt{k / m}}
$$
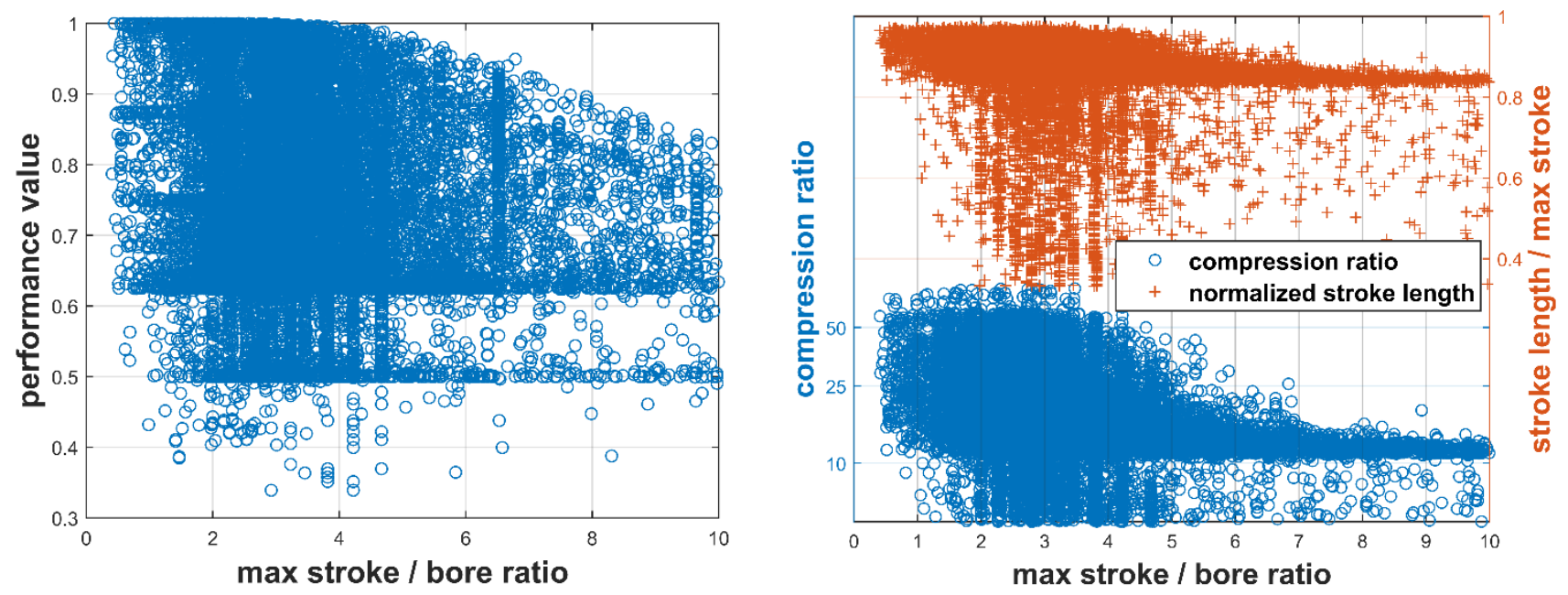

Figure 117: Effects of maximum stroke to bore ratio on performance, compression ratio, and stroke length. Performance is calculated based on the weight profiles given in Figure 85, but without target power.
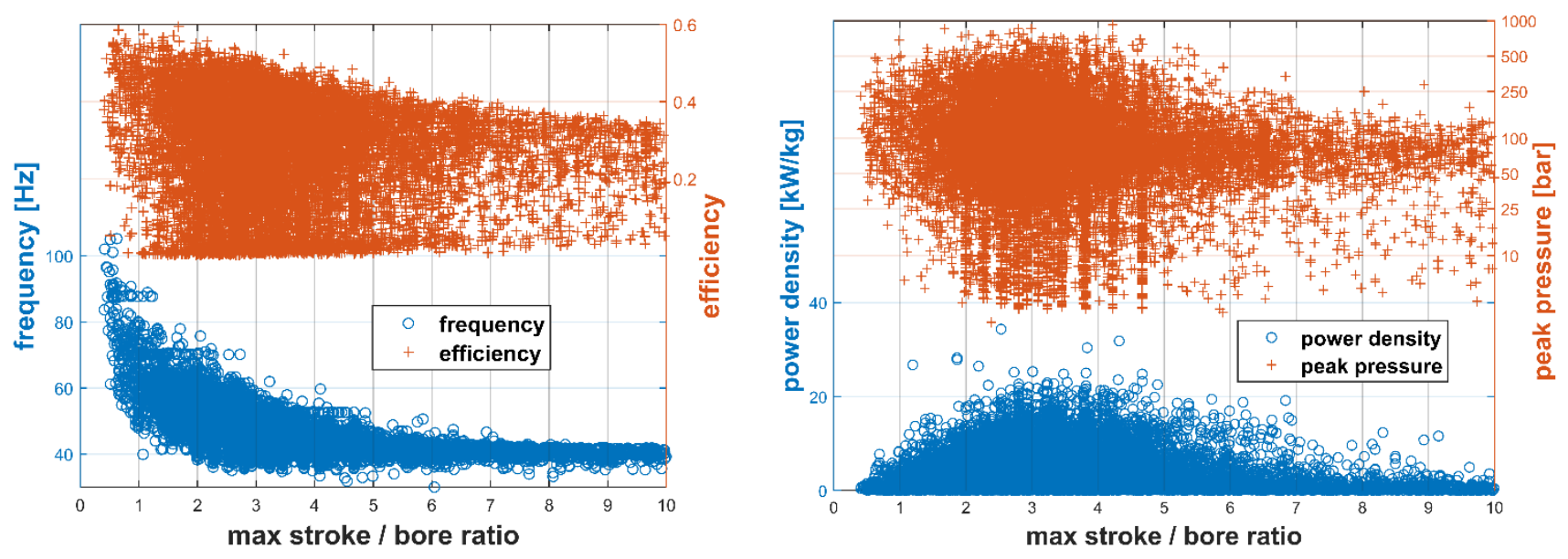

Figure 118: Effects of maximum stroke to bore ratio on frequency, efficiency, power density, and peak pressure. 

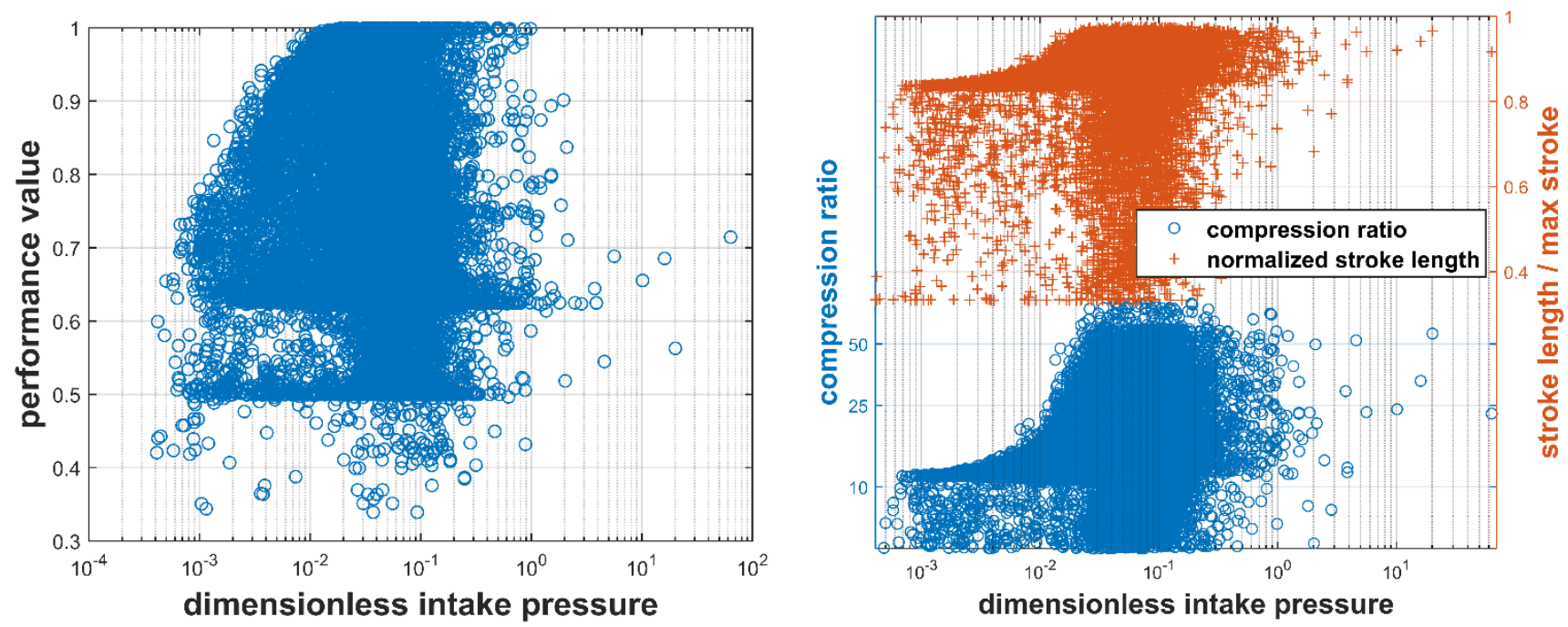

Figure 119: Effects of dimensionless intake pressure on performance, compression ratio, and stroke length. Performance is calculated based on the weight profiles given in Figure 85, but without target power.
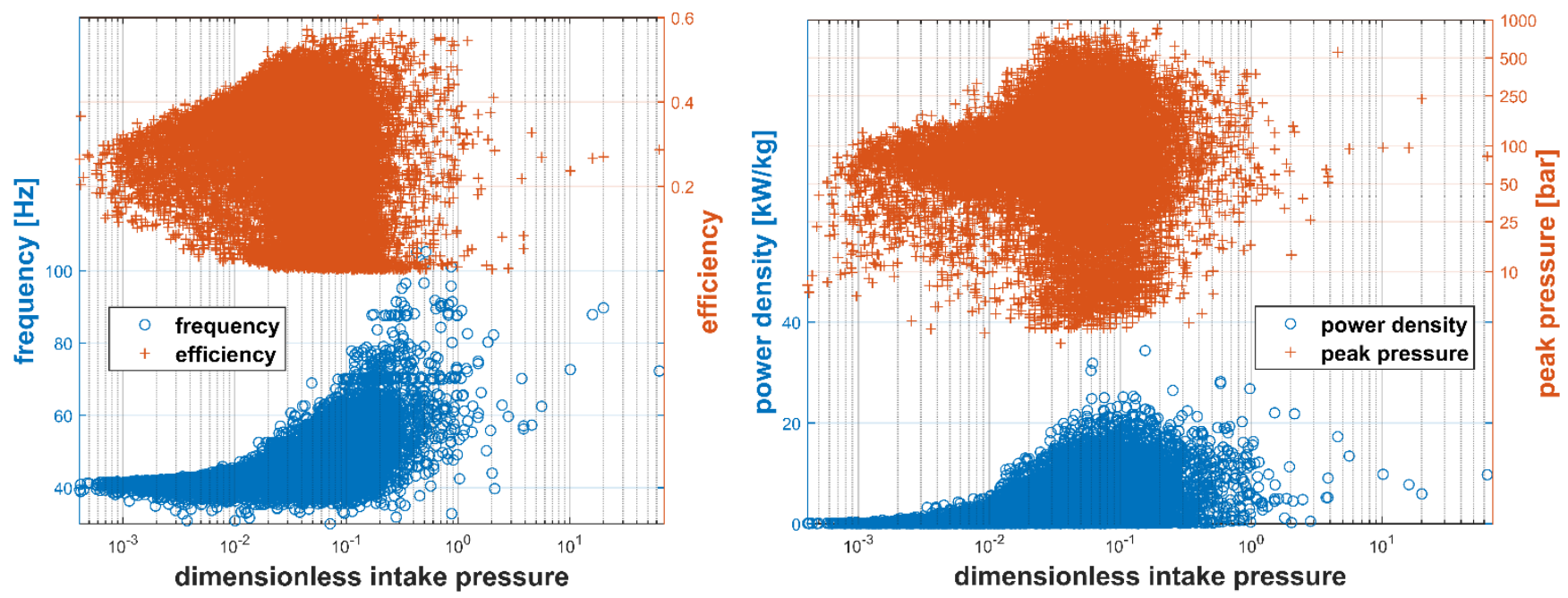

Figure 120: Effects of dimensionless intake pressure on frequency, efficiency, power density, and peak pressure. 

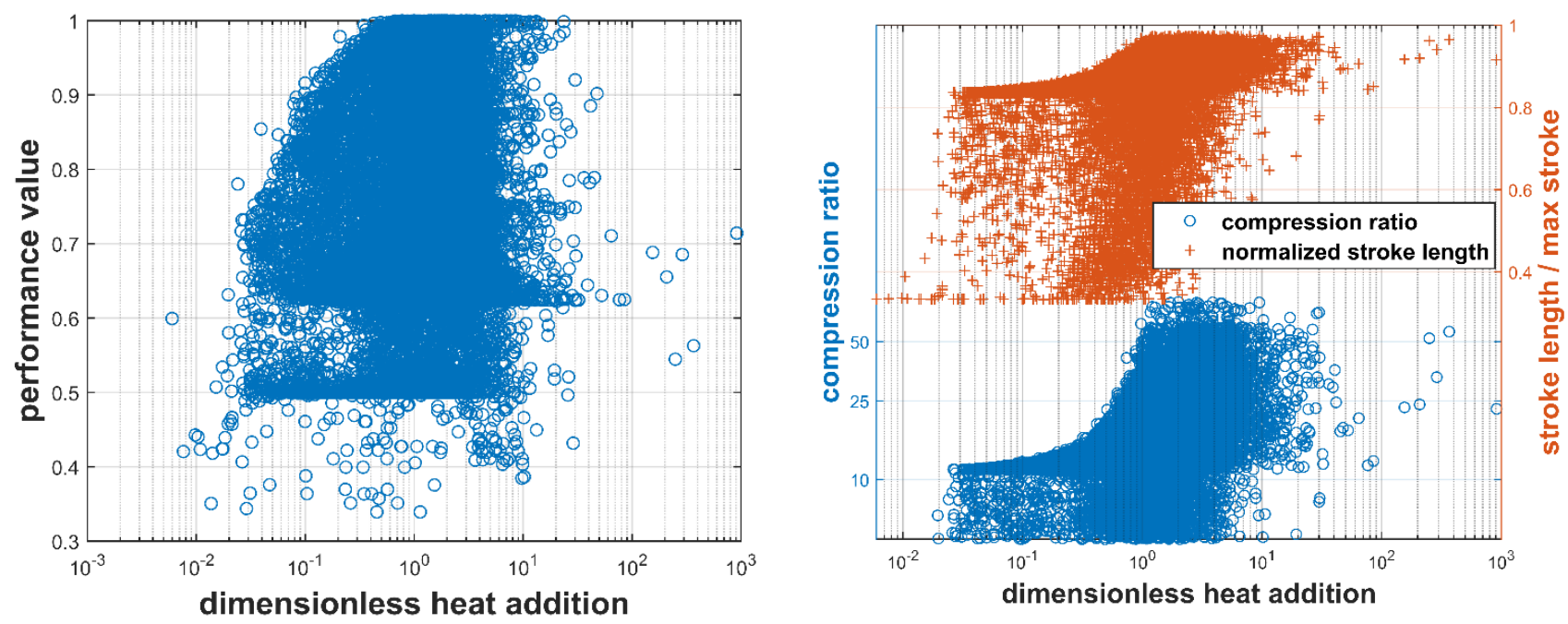

Figure 121: Effects of dimensionless heat addition on performance, compression ratio, and stroke length. Performance is calculated based on the weight profiles given in Figure 85, but without target power.
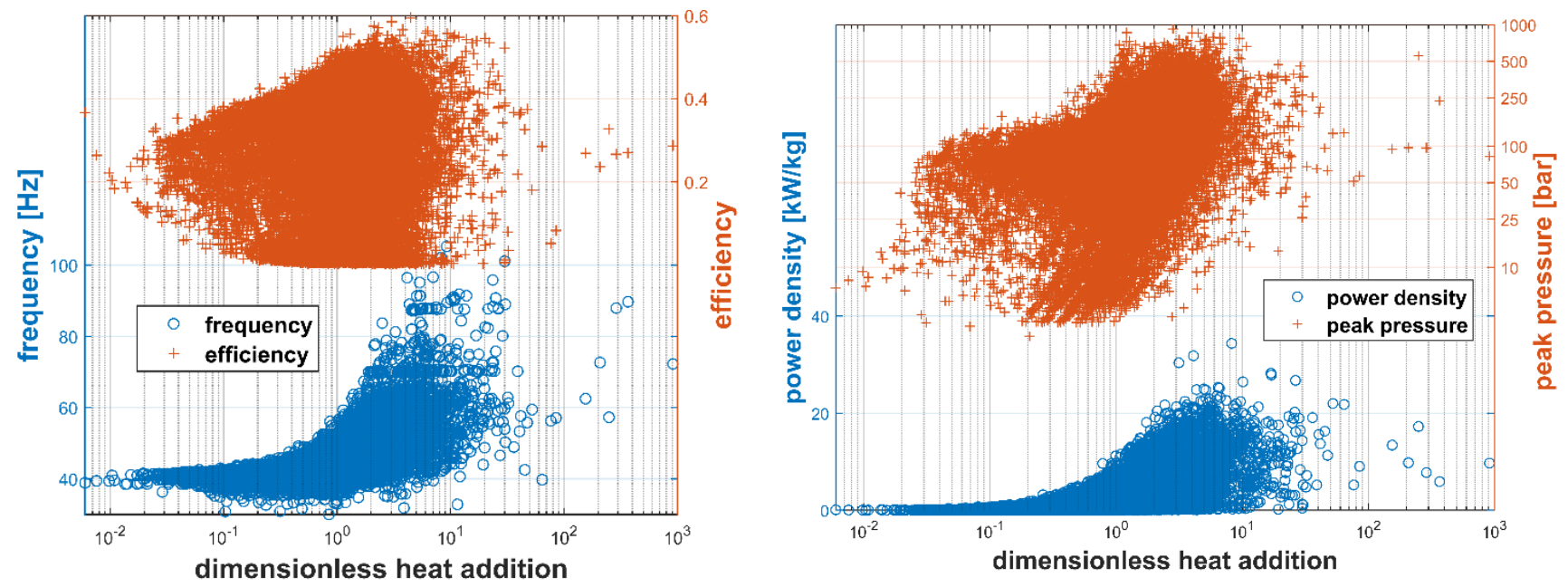

Figure 122: Effects of dimensionless heat addition on frequency, efficiency, power density, and peak pressure. 

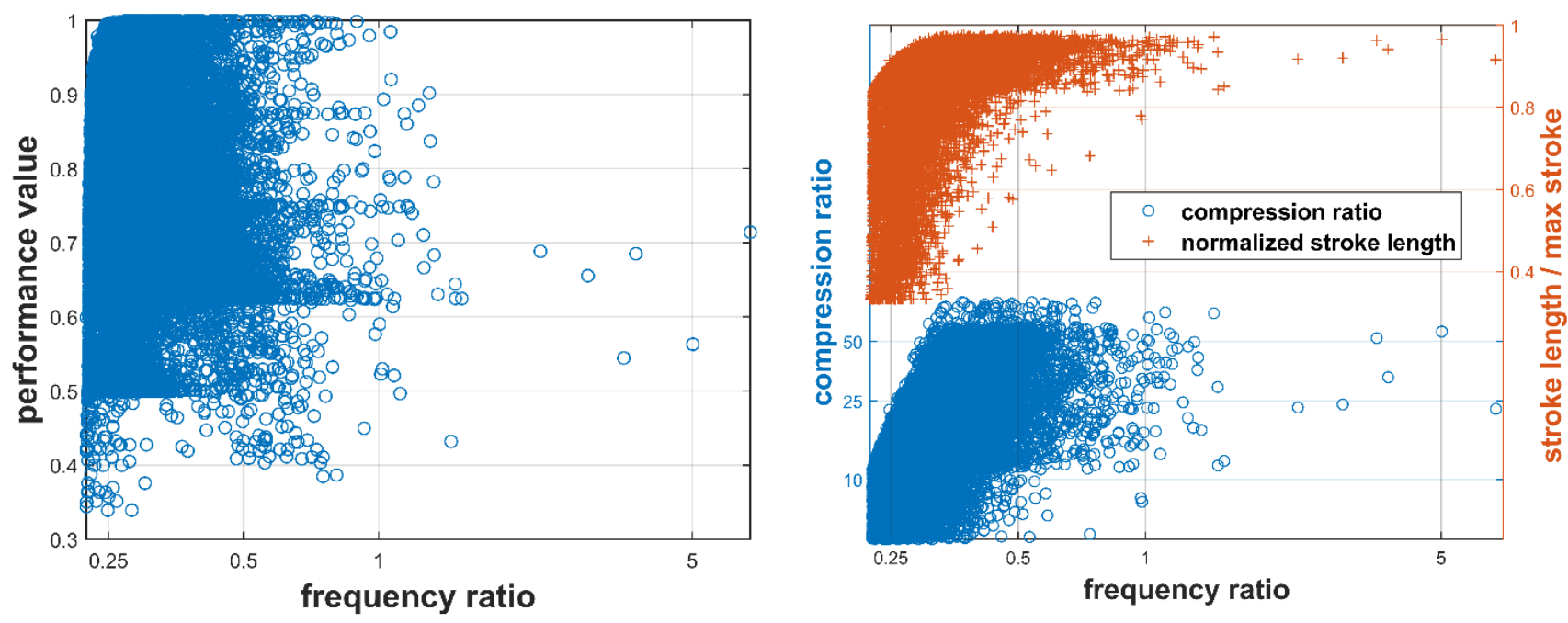

Figure 123: Effects of frequency ratio on performance, compression ratio, and stroke length. Performance is calculated based on the weight profiles given in Figure 85, but without target power.
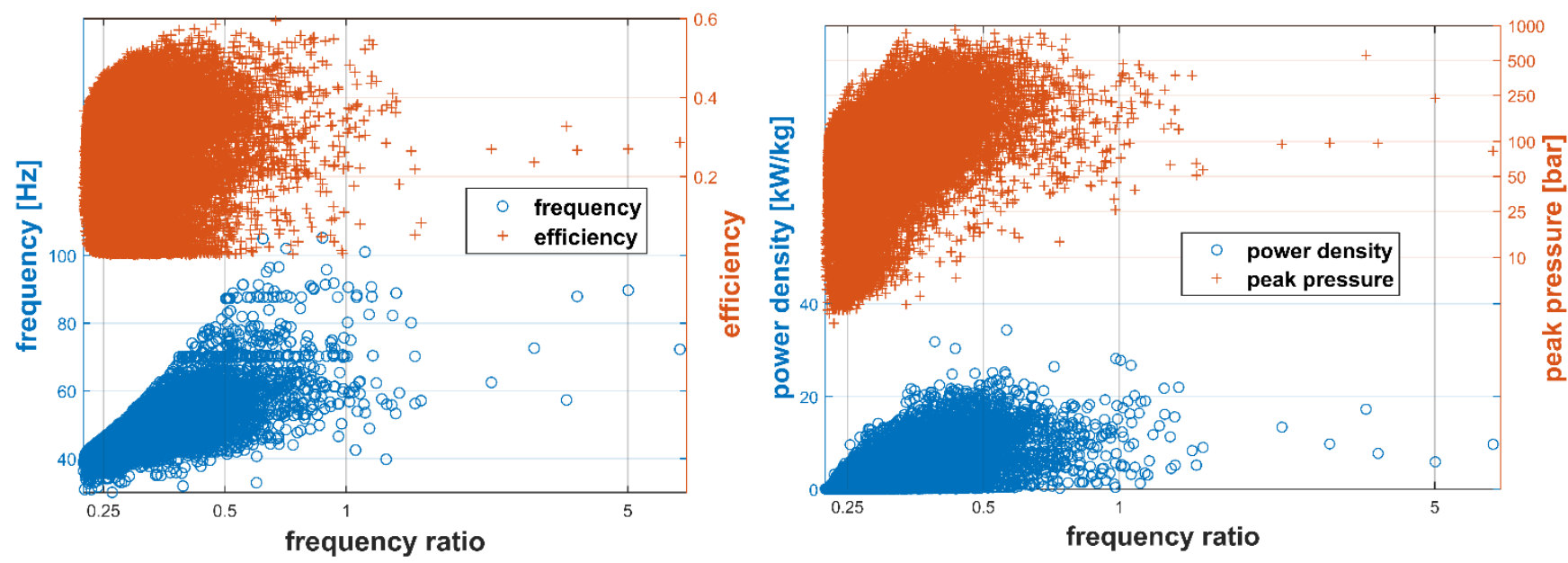

Figure 124: Effects of frequency ratio on frequency, efficiency, power density, and peak pressure. 